\title{
Discovery and measurement of the Higgs boson in the WW decay channel
}

\author{
David Christopher Hall \\ St Catherine's College, University of Oxford
}
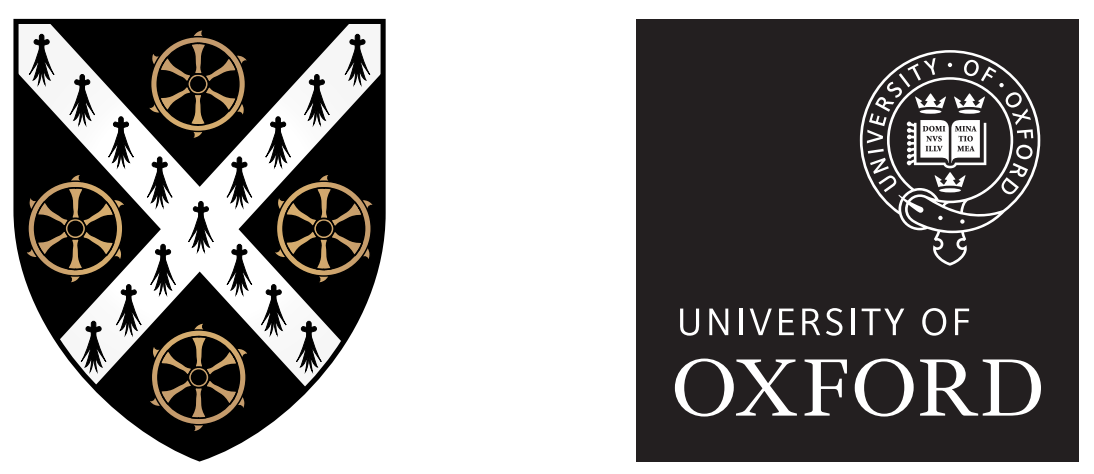

Submitted in partial fulfilment of the requirements for the degree of Doctor of Philosophy

Trinity 2014 


\section{Abstract}

In the Standard Model of particle physics, the non-zero masses of the $W$ and $Z$ bosons and the fermions are generated through interactions with the Higgs field, excitations of which correspond to Higgs bosons. Thus, the experimental discovery of the Higgs boson is of prime importance to physics, and would confirm our understanding of fundamental mass generation.

This thesis describes a search for the $g g \rightarrow H \rightarrow W W \rightarrow \ell \nu \ell \nu$ process of Higgs boson production and decay. It uses the LHC Run I dataset of $p p$ collisions recorded by the ATLAS detector, which corresponds to an integrated luminosity of $4.5 \mathrm{fb}^{-1}$ at $\sqrt{s}=7 \mathrm{TeV}$ and $20.3 \mathrm{fb}^{-1}$ at $\sqrt{s}=8 \mathrm{TeV}$. An excess of events is observed with a significance of 4.8 standard deviations, which is consistent with Higgs boson production. The significance is extended to 6.1 standard deviations when the vector boson fusion production process is included. The measured signal strength is $1.11_{-0.21}^{+0.23}$ at $m_{H}=125 \mathrm{GeV}$. A cross section measurement of $W W$ production, a major background to this search, is also presented using the $\sqrt{s}=7 \mathrm{TeV}$ dataset only. 


\section{Preface}

As a DPhil research topic, the $H \rightarrow W W$ analysis has proven to be a baptism of fire. It is the most complicated of the three "discovery channels", ${ }^{1}$ as it involves a variety of physics objects and requires a good understanding of many difficult backgrounds. As such, the analysis took huge effort from a large number of individuals. My role focussed on theoretical aspects of the signal and background modelling, and these parts shall be emphasised. I contributed to multiple iterations of the analysis [1-8], though the version presented here is unpublished at the time of writing [9]. I also co-authored the third Yellow Report produced by the LHC Higgs Cross Section Working Group [10].

When I began the degree in October 2010, there was no direct evidence for a Higgs boson. This thesis is written from a personal perspective and motivates a low mass search by electroweak fits, when in fact this aspect was motivated later by observations of a resonance in the $\gamma \gamma$ and $Z Z$ channels. ${ }^{2}$ Also, an advanced search strategy is described, though the discovery of $H \rightarrow W W$ was actually a gradual process with multiple iterations of blinding, optimising and unblinding the analysis. As more data were recorded and the analysis was enhanced, the results improved.

Early on, I gained relevant insight by performing multiple $W W$ cross section measurements [12-15]. My main contribution was a jet veto correction factor applied to the $W W$ signal, which reduces the dominant uncertainty in the analysis. This measurement shall be described when considering the $W W$ background to the $H \rightarrow W W$ search.

To qualify for authorship within the ATLAS collaboration, I performed Run Control shifts. I also worked within the Versatile Link project [16] to investigate radiation hardened optical components for the HL-LHC. As this research does not easily relate to the Higgs boson, it is excluded from this thesis. However, I have published articles on the radiation tolerance of optical fibres [17] and their connectors [18].

\footnotetext{
${ }^{1}$ The $\gamma \gamma, Z Z$ and $W W$ decay channels quickly gave sensitivity to the Higgs boson ultimately discovered.

${ }^{2}$ Dedicated high mass searches for $H \rightarrow W W$ have also been performed [11].
} 


\section{Acknowledgements}

Over the last 3.5 years, I have received help and support from many individuals. First and foremost is my supervisor, Chris Hays, whom I was fortunate to work closely with. I thank him for his patience, and for our enlightening conversations on perturbative QCD, the brewing of fine beers and how to explain the hierarchy problem with plush toys.

The ATLAS $H \rightarrow W W$ analysis group is comprised of many students, post-docs and research fellows, all collaborating to achieve a common goal. I thank Jianming Qian, Biagio Di Micco, Pierre Savard, Tatsuya Masubuchi, Christian Schmitt and Corrinne Mills for successfully convening such a complex analysis. There are too many members of the analysis team for me to mention here, but I would particularly like to thank Jonathan Long, Keisuke Yoshihara, Olivier Arnaez and Magda Chelstowska. For their help and guidance in my work on theoretical uncertainties, I thank Chris Hays, Biagio Di Micco, Justin Griffiths, Bob Kehoe and Sara Diglio. I also thank the Higgs Cross Section Working Group for their feedback on the ggF jet binning studies, in particular Andrea Banfi, Gavin Salam and Frank Tackmann. I thank MCnet for their series of summer schools, the SHERPA authors for releasing a version that enabled the $W \gamma^{*}$ sample to be produced, and Stefano Frixione for his advice during my time at CERN.

For the $7 \mathrm{TeV} W W$ cross section measurement, I would like to thank Marc-Andre Pleier and Matthias Schott in their role as analysis convenors. I also thank Shu Li and Yusheng $\mathrm{Wu}$, when we were endlessly poring over cutflows.

Within the Oxford ATLAS group, Todd Huffman and Tony Weidberg provided excellent supervision during my ATLAS service work project, and an introduction to Belgian nuclear reactors and beer (and why they should not be mixed). Thanks must also go to Gemma Wooden for getting me started in the $W W$ and $H \rightarrow W W$ analyses at a time when she was busy finishing her DPhil. I am truly grateful to Alex Dafinca and Lucy Kogan for sharing this experience from the start, and Jacob Howard for his overwhelming computing knowledge. I also thank Chris Y, Craig, Ellie, Jim, Kate, Mireia, Rob, Sarah and Shaun for office banter. Finally, I thank Sue Geddes and Kim Proudfoot 
for their help with administrative matters, and Oxford IT support for maintaining an excellent computer farm (on which I munched through 100,000 CPU hours).

I feel very lucky to have been a member of St Catherine's College during my DPhil. I've met such a variety of interesting characters in the Catz MCR, particularly in my 1st and 3rd years; it really has been a defining aspect of my time at Oxford University. Thanks for such great memories and I look forward to many more to come.

Of course, I also thank my parents, the rest of my family and my close friends for helping me through the difficult times.

Lastly, I thank the STFC for financially supporting me for the majority of the degree, and ACEOLE for granting me a bursary to speak at the TWEPP-11 conference in Vienna. I am also indebted to St Catherine's College for awarding me the College Science Scholarship in my 3rd year and a Light Senior Scholarship in my 4th year. They also provided other funding at various points throughout the degree, e.g. enabling me to attend the CLASHEP-13 school in Peru. 
"By believing passionately in something that still does not exist, we create it. The nonexistant is whatever we have not sufficiently desired."

- Nikos Kazantzakis 


\section{Contents}

$\begin{array}{ll}\text { Introduction } & 1\end{array}$

1 Theoretical motivation 3

1.1 The Standard Model of particle physics . . . . . . . . . . . . . . 3

1.2 Electroweak unification . . . . . . . . . . . . . . . . . 5

1.3 Properties of the Higgs boson . . . . . . . . . . . . . . . . 11

1.4 Pre-LHC constraints on the Higgs boson mass . . . . . . . . . . . . 13

2 Computational techniques for the LHC 17

2.1 Quantum chromodynamics . . . . . . . . . . . . . . 17

2.2 Monte Carlo event generation . . . . . . . . . . . . . . . . . . . . 22

2.3 Jet algorithms . . . . . . . . . . . . . . . . . . . . . . . . . . . . 29

3 The ATLAS experiment 31

3.1 The Large Hadron Collider . . . . . . . . . . . . . . . . . . . . . 31

$3.2 p p$ collision data . . . . . . . . . . . . . . . . . . . . . . . . 33

3.3 The ATLAS detector . . . . . . . . . . . . . . . 35

4 Overview of the $\mathrm{H} \rightarrow \mathrm{WW}$ analysis $\quad 44$

4.1 Experimental signature . . . . . . . . . . . . . . . . 44

4.2 Reconstruction of physics objects . . . . . . . . . . . . . . . . 46

4.3 Event selection criteria . . . . . . . . . . . . . . . 62

5 Signal modelling $\quad 78$

5.1 Jet-binned cross sections . . . . . . . . . . . . . . . . . . . 79

5.2 Monte Carlo modelling . . . . . . . . . . . . . . . . 88

6 WW measurement and modelling $\quad 96$

6.1 Cross section measurement in the 0 -jet bin . . . . . . . . . . . . . 97

6.2 Background estimation for $H \rightarrow W W$ search . . . . . . . . . . . . . 110 
7 Other backgrounds $\quad 119$

$7.1 W+$ jet and dijet . . . . . . . . . . . . . . . 120

7.2 Non- $W W$ diboson . . . . . . . . . . . . . . . . . . . . . . . . . . . . 128

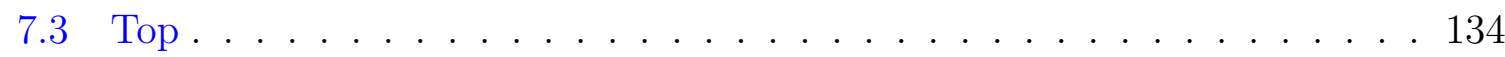

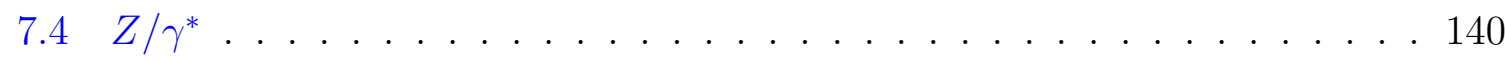

7.5 Summary of normalisation factors . . . . . . . . . . . . . . 146

8 Experimental results $\quad 148$

8.1 Systematic uncertainties . . . . . . . . . . . . . . . . . 148

8.2 Statistical model . . . . . . . . . . . . . . . . . . . . . . 151

8.3 Results . . . . . . . . . . . . . . . . . . . . 158

9 Status of Higgs physics $\quad 169$

9.1 Properties of the discovered Higgs boson . . . . . . . . . . . . . . . . . 169

9.2 Theoretical implications . . . . . . . . . . . . . . . . . . . 174

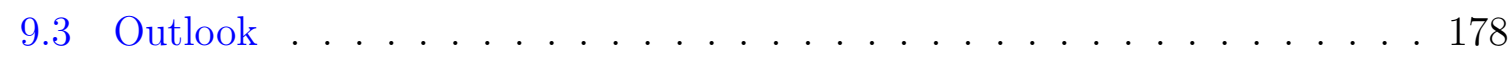

10 Conclusions $\quad 181$

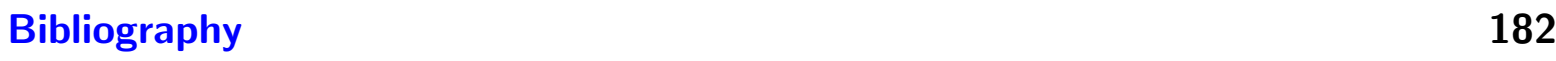




\section{Introduction}

The Standard Model of particle physics describes the behaviour of sub-atomic particles. Since its formulation in the 1970s, it has experienced unparalleled success in modelling a wide range of phenomena; no experimental result within the remit of the Standard Model is currently considered to significantly contradict its validity. ${ }^{3}$ However, there are a number of physical phenomena that the Standard Model is unable to describe: gravitational attraction between massive objects, the observed asymmetry between matter and antimatter in the Universe, and astronomical evidence for dark matter and the cosmological constant.

A crucial aspect of the Standard Model is how non-zero masses are imparted to fundamental particles. These are forbidden by underlying symmetries of the theory, though remain an experimental fact; for example, atoms could not form if the electron did not possess mass. This is achieved via interactions with a ubiquitous Higgs field, excitations of which correspond to Higgs bosons. As the only undiscovered particle of the Standard Model, the discovery of the Higgs boson is of utmost importance to particle physics: it would complete our knowledge of the Standard Model, and in particular confirm the mechanism of mass generation. As such, it was a primary goal of the LHC physics program, which began in 2010 .

This thesis describes the search, discovery and measurement of the Higgs boson using proton-proton collision data recorded by the ATLAS experiment at CERN. This is accomplished by searching for collisions where a Higgs boson is produced and subsequently decays to two $W$ bosons, each of which decay to an electron or muon and a neutrino (i.e. $H \rightarrow W W \rightarrow \ell \nu \ell \nu)$. This search suffers from large experimental backgrounds, such as continuum $W W$ production, which must be accurately modelled to yield sensitivity to the Higgs boson.

\footnotetext{
${ }^{3}$ Observation of neutrino oscillations required neutrino masses to be manually added to the Standard Model. It is widely believed that their relatively small masses will be explained by new physics.
} 
First, the theoretical motivation for the Higgs boson is presented in Chapter 1. Then, Chapter 2 outlines some important concepts related to making precise predictions within the Standard Model, which shall be referred to throughout the thesis. The experimental setup of the LHC and the ATLAS detector are described in Chapter 3.

Focus then moves to the data analysis itself. Chapter 4 offers an overview of the entire $H \rightarrow W W$ analysis, detailing the selection of Higgs boson signal events and the rejection of backgrounds. Following this, signal modelling is described in Chapter 5, $W W$ background modelling is described in Chapter 6 (including a dedicated cross section measurement), and the modelling of other backgrounds is described in Chapter 7. The experimental results are presented and discussed in Chapter 8. Finally, in Chapters 9 and 10, we draw conclusions from the results of this analysis and of others conducted simultaneously at the LHC, and consider the outlook of Higgs physics. 


\section{Chapter 1}

\section{Theoretical motivation}

The Standard Model (SM) of particle physics is the theory of sub-atomic particles. It is the culmination of many decades of progress in both experimental and theoretical quantum physics during the 20th century. It has enjoyed unparalleled success in describing a wide range of phenomena, which have been experimentally verified to an extraordinary degree of precision.

A crucial aspect of the SM is the generation of non-zero particle masses, which are forbidden by underlying symmetries of the theory but remain an experimental fact. They are generated by the Higgs mechanism of electroweak symmetry breaking, which also predicts the existence of a massive scalar particle, the Higgs boson, whose mass is a free parameter of the theory. As the only undiscovered particle of the SM, the discovery of the Higgs boson was a primary goal of the LHC physics program, which began in 2010.

A brief introduction to the SM is given in Section 1.1, outlining the particle content and interactions of the theory. In Section 1.2, electroweak symmetry breaking is described in detail. Then, some properties of the Higgs boson are described in Section 1.3, and the constraints upon its mass prior to the LHC are detailed in Section 1.4.

\subsection{The Standard Model of particle physics}

The SM is a gauge quantum field theory describing the kinematics and interactions of subatomic particles [19-24]. The dynamics of such a theory are determined by the symmetries respected by its Lagrangian. The SM is invariant under local transformations of the $\mathrm{SU}(3) \times \mathrm{SU}(2) \times \mathrm{U}(1)$ gauge group, resulting in the strong, weak and electromagnetic forces of nature. Additionally, invariance under global transformations of the Poincaré 


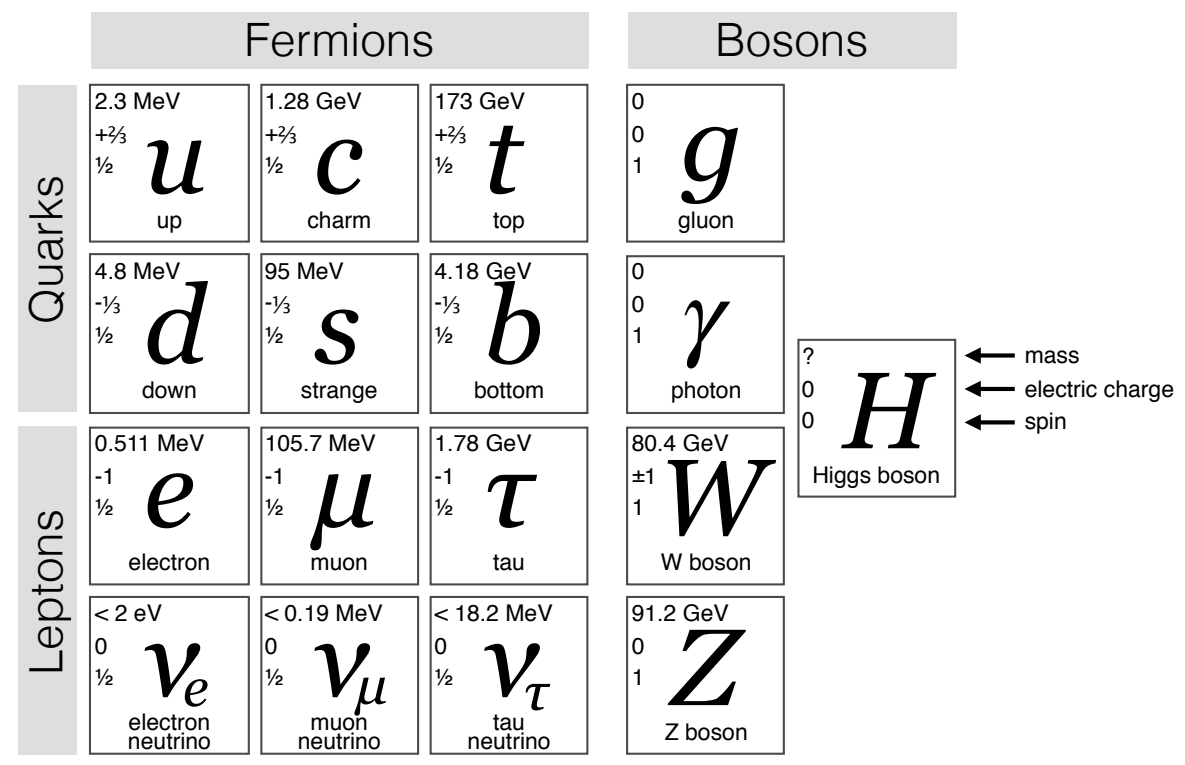

Figure 1.1: The particle content of the SM, with masses from [26]. Constraints upon the mass of the Higgs boson are described in Section 1.4.

group ensures the theory is identical in all inertial frames of reference, as asserted by special relativity.

Each constituent gauge theory of the SM describes the dynamics of a force of nature, which is mediated by a number of gauge bosons and couples to a conserved current, in accordance with Noether's theorem [25]. Quantum chromodynamics (QCD) of SU(3) describes the strong interaction, is mediated by eight gluons, and couples to colour charge. Quantum electrodynamics (QED) of $\mathrm{U}(1)$ describes the electromagnetic interaction, is mediated by the photon, and couples to electric charge. The weak interaction is mediated by the massive $W^{ \pm}$and $Z$ bosons and is best understood within the context of the electroweak (EW) theory, a unification of the electromagnetic and weak interactions. A theory of gravity is not included in the SM. Significantly, the gauge groups of the strong and weak interactions are non-abelian. Physically, this means that the gauge bosons are themselves charged and therefore experience self-interactions.

The elementary particles of the SM are summarised in Figure 1.1. They are categorised into bosons (integer spin) and fermions (half-integer spin). In addition to the gauge bosons introduced above, the Higgs boson is a by-product of electroweak symmetry breaking (described in Section 1.2) and couples to mass. The twelve flavours of fermions are categorised according to the interactions they experience, or equivalently the charges they possess: quarks (strong, electromagnetic, weak), charged leptons (electromagnetic, 
weak) and neutrinos (weak). The fermions are also arranged in three generations of increasing mass. Massive particles can decay into less massive particles, while obeying the conservation laws of the SM. Fermions have an associated antiparticle with identical mass but inverted internal quantum numbers. Isolated quarks are not observed; they form colourless composite particles called hadrons.

\subsection{Electroweak unification}

The first theory of weak interactions was a four-point interaction with Fermi coupling constant $G_{\mathrm{F}}=1.166 \times 10^{-5} \mathrm{GeV}^{-2}$. Although successful in describing low energy phenomena, such as nuclear $\beta$-decay and muon decay, at energies above $\sim 300 \mathrm{GeV}$ the theory predicted cross sections which violate unitarity [19].

The solution was to introduce charged vector bosons ( $W^{ \pm}$bosons) to mediate the weak interaction, similar to the exchange of photons in QED. However, unlike QED, the weak interaction is short ranged and therefore its exchange bosons must be massive. Since the propagator for a particle of mass $m$ and momentum $p$ contains a factor $1 /\left(p^{2}-m^{2}\right)$, in the low energy limit we can relate to Fermi's theory and identify that $G_{\mathrm{F}} \sim g^{2} / m_{W}^{2}$, where $g$ is the coupling of the vector boson. Thus, at low energies, the strength of the weak interaction is suppressed by the mass of the exchange boson.

At this time, there were two key obstacles to unifying the electromagnetic and weak interactions. First, the discovery of parity violation in cobalt-60 $\beta$-decay implied the weak interaction has a $\mathrm{V}-\mathrm{A}$ structure, whereas QED has a pure V structure [27]. ${ }^{1}$ Second, the $W^{ \pm}$bosons are massive whilst photons are massless. This was a major problem because gauge bosons are inherently massless. ${ }^{2}$ In fact, fermion masses were also forbidden by the chiral nature of the weak interaction, but were known to exist. ${ }^{3}$

Glashow's proposal of an $\mathrm{SU}(2)_{\mathrm{L}} \times \mathrm{U}(1)_{Y}$ group was a major step forward [21]. This model describes three gauge fields $\left(W_{\mu}^{1}, W_{\mu}^{2}, W_{\mu}^{3}\right)$ which couple to weak isospin $T$ with strength $g$, and a single gauge field $B_{\mu}$ which couples to weak hypercharge $Y$ with

\footnotetext{
${ }^{1}$ Five bilinear covariants can be constructed from the Dirac $\gamma$ matrices, which are named according to how they transform under parity: scalar, pseudoscalar, vector, axial vector and tensor.

${ }^{2}$ Consider the gauge transformation of a Yang-Mills gauge field $\boldsymbol{W}_{\mu} \rightarrow \boldsymbol{W}_{\mu}-\partial_{\mu} \boldsymbol{\alpha}(x)-g\left[\boldsymbol{\alpha}(x) \times \boldsymbol{W}_{\mu}\right]$. Clearly the mass term $-\frac{1}{2} m^{2} \boldsymbol{W}_{\mu} \cdot \boldsymbol{W}^{\mu}$ is not gauge invariant, and hence the gauge boson is massless.

${ }^{3}$ Consider a spinor as the sum of its left- and right-handed chiral states $\psi=\psi_{\mathrm{L}}+\psi_{\mathrm{R}}$. Then the Dirac mass term is $-m \bar{\psi} \psi=-m\left(\bar{\psi}_{\mathrm{R}} \psi_{\mathrm{L}}+\bar{\psi}_{\mathrm{L}} \psi_{\mathrm{R}}\right)$. For a chiral theory, $\psi_{\mathrm{L}}$ and $\psi_{\mathrm{R}}$ behave differently under gauge transformations and thus the mass term is not gauge invariant.
} 


\begin{tabular}{ccccccc}
\hline & & $T$ & $T_{3}$ & $Y$ & $Q$ \\
\hline$\left(\begin{array}{c}\nu_{e} \\
e\end{array}\right)_{\mathrm{L}}$ & $\left(\begin{array}{c}\nu_{\mu} \\
\mu\end{array}\right)_{\mathrm{L}}$ & $\left(\begin{array}{c}\nu_{\tau} \\
\tau\end{array}\right)_{\mathrm{L}}$ & $\frac{1}{2}$ & $+\frac{1}{2}$ & -1 & 0 \\
$\nu_{e \mathrm{R}}$ & $\nu_{\mu_{\mathrm{R}}}$ & $\nu_{\tau \mathrm{R}}$ & 0 & 0 & 0 & 0 \\
$e_{\mathrm{R}}$ & $\mu_{\mathrm{R}}$ & $\tau_{\mathrm{R}}$ & 0 & 0 & -2 & -1 \\
\hline$\left(\begin{array}{c}u \\
d^{\prime}\end{array}\right)_{\mathrm{L}}$ & $\left(\begin{array}{c}c \\
s^{\prime}\end{array}\right)_{\mathrm{L}}$ & $\left(\begin{array}{c}t \\
b^{\prime}\end{array}\right)_{\mathrm{L}}$ & $\frac{1}{2}$ & $+\frac{1}{2}$ & $+\frac{1}{3}$ & $+\frac{2}{3}$ \\
$u_{\mathrm{R}}$ & $c_{\mathrm{R}}$ & $t_{\mathrm{R}}$ & 0 & 0 & $+\frac{1}{3}$ & $-\frac{1}{3}$ \\
$d_{\mathrm{R}}$ & $s_{\mathrm{R}}$ & $b_{\mathrm{R}}$ & 0 & 0 & $-\frac{2}{3}$ & $-\frac{1}{3}$ \\
\hline
\end{tabular}

Table 1.1: The weak isospin $T$, weak hypercharge $Y$ and electric charge $Q$ of the fermions. In charged currents, the states that couple to $u$-type quarks are superpositions of $d$-type quarks and are denoted with a prime. Although right-handed neutrinos are decoupled, recent observations of neutrino oscillations suggest these might exist.

strength $g^{\prime}$. The subscript L indicates that only left-handed chiral particles couple to the $W_{\mu}^{i}$ fields, explaining the $\mathrm{V}-\mathrm{A}$ nature of the weak interaction whilst preserving QED. The physical gauge fields are obtained through the mixing of these fields

$$
\begin{aligned}
W_{\mu}^{ \pm} & =\left(W_{\mu}^{1} \mp i W_{\mu}^{2}\right) / \sqrt{2} \\
Z_{\mu} & =\cos \theta_{\mathrm{W}} W_{\mu}^{3}-\sin \theta_{\mathrm{W}} B_{\mu} \\
A_{\mu} & =\sin \theta_{\mathrm{W}} W_{\mu}^{3}+\cos \theta_{\mathrm{W}} B_{\mu}
\end{aligned}
$$

where

$$
\cos \theta_{\mathrm{W}}=g / \sqrt{g^{2}+g^{\prime 2}} \quad \text { and } \quad \sin \theta_{\mathrm{W}}=g^{\prime} / \sqrt{g^{2}+g^{\prime 2}} .
$$

We identify $W_{\mu}^{ \pm}$with the $W^{ \pm}$bosons, $A_{\mu}$ with the photon and $Z_{\mu}$ with a new neutral $Z$ boson. Weak neutral currents were later confirmed experimentally [28].

Glashow's $\mathrm{SU}(2)_{\mathrm{L}} \times \mathrm{U}(1)_{Y}$ theory therefore predicts the interaction of fermions, in left-handed SU(2) doublets and right-handed SU(2) singlets (see Table 1.1), with $W^{ \pm}, Z$ and $\gamma$ exchange bosons. Gauge boson self-interactions are also expected due to the nonabelian nature of the EW theory. The $W^{ \pm}$bosons couple to weak isospin $T$ with strength $g$, the $Z$ boson couples vectorially to $c_{\mathrm{V}}$ and axially to $c_{\mathrm{A}}$ with strength $g /\left(2 \cos \theta_{\mathrm{W}}\right)$, 


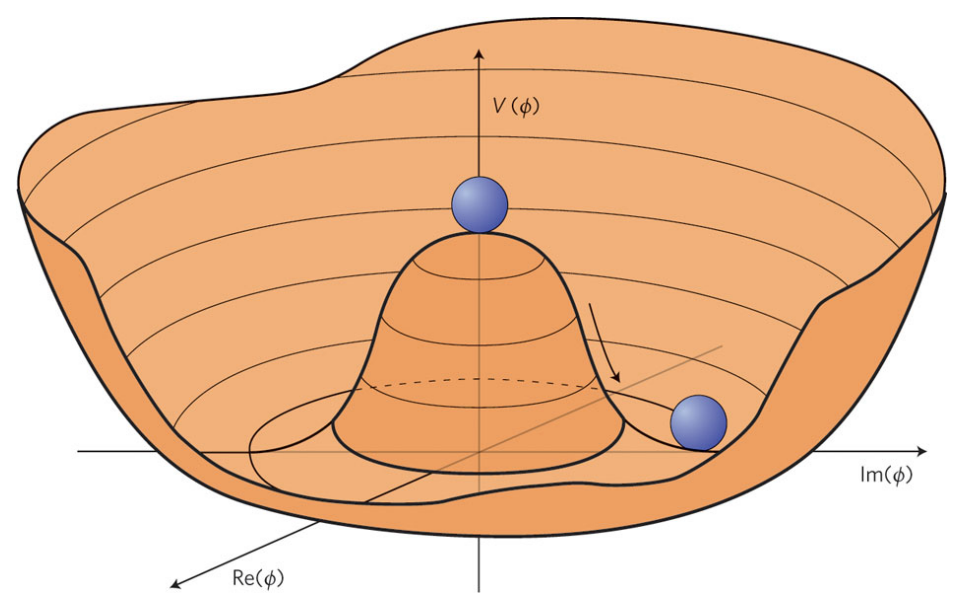

Figure 1.2: The sombrero potential, in which a vacuum state must be arbitrarily chosen, spontaneously breaking the symmetry of the underlying Lagrangian [29]. Fluctuations in the azimuthal direction correspond to a massless Nambu-Goldstone boson. Fluctuations in the radial direction correspond to a massive Higgs boson. Reprinted by permission from Macmillan Publishers Ltd: Nature Physics 7, 2 (2011), copyright (2011).

and the photon couples to electric charge $Q$ with strength $e=g \sin \theta_{\mathrm{W}}$, where

$$
\begin{aligned}
c_{\mathrm{V}} & =T_{3}-2 Q \sin ^{2} \theta_{\mathrm{W}}, \quad c_{\mathrm{A}}=T_{3} \\
Q & =T_{3}+\frac{Y}{2}
\end{aligned}
$$

Unfortunately, it was necessary to explicitly break the symmetry by adding mass terms for the $W^{ \pm}$and $Z$ bosons by hand. Initial attempts to invoke a mechanism of spontaneous symmetry breaking (SSB) were hindered by the Goldstone theorem.

\subsubsection{The Goldstone theorem}

SSB arises when the vacuum state does not respect the symmetry in question. This can occur when a field acquires a non-zero vacuum expectation value. To see this, consider a complex scalar field $\phi$ described by the Lagrangian

$$
\mathcal{L}=\left(\partial_{\mu} \phi^{\dagger}\right)\left(\partial^{\mu} \phi\right)+\mu^{2} \phi^{\dagger} \phi-\lambda\left(\phi^{\dagger} \phi\right)^{2}
$$

with positive $\mu^{2}$ and $\lambda$, giving a sombrero potential (Figure 1.2). Although $\mathcal{L}$ is invariant under global $\mathrm{U}(1)$ transformations $\phi \rightarrow e^{-i \alpha} \phi$, there are infinite degenerate vacua 
$\phi=\mu e^{-i \theta} / \sqrt{2 \lambda}$ that are not invariant. In order to interact with the system, a single vacuum must be arbitrarily chosen, spontaneously breaking the $\mathrm{U}(1)$ symmetry.

The Goldstone theorem states that SSB of a continuous global symmetry will lead to the existence of a number of massless scalar Nambu-Goldstone bosons [30]. This can be seen by considering radial and azimuthal excitations, $h(x)$ and $\theta(x)$, about the vacuum

$$
\phi(x)=\frac{1}{\sqrt{2}}[v+h(x)] e^{-i \theta(x) / v}
$$

where $v=\mu / \sqrt{\lambda}$. When substituted into (1.7), we get

$$
\mathcal{L}=\frac{1}{2} \partial_{\mu} \theta \partial^{\mu} \theta+\frac{1}{2} \partial_{\mu} h \partial^{\mu} h-\mu^{2} h^{2}+\ldots
$$

where the dots denote terms neither kinetic nor mass. We identify a massless NambuGoldstone boson (the $\theta$-mode) and a Higgs boson of mass $\sqrt{2} \mu$ (the $h$-mode).

In order to explain massive $W^{ \pm}$and $Z$ bosons, the electroweak symmetry must be broken. But the Goldstone theorem suggested that this would predict massless scalar bosons, which were not experimentally observed.

\subsubsection{The Higgs mechanism}

However, when SSB of a continuous local symmetry is studied, something remarkable happens. The Nambu-Goldstone bosons of the theory are 'eaten' by the gauge bosons, giving them mass. The associated degrees of freedom appear as longitudinal components of the massive gauge bosons. This is known as the Higgs mechanism [31-35].

Consider the Lagrangian for a U(1) gauge theory with a sombrero potential

$$
\mathcal{L}=\left(D_{\mu} \phi\right)^{\dagger}\left(D^{\mu} \phi\right)-\frac{1}{4} F_{\mu \nu} F^{\mu \nu}+\mu^{2} \phi^{\dagger} \phi-\lambda\left(\phi^{\dagger} \phi\right)^{2}
$$

where $D_{\mu}=\partial_{\mu}+i q A_{\mu}$ is the covariant derivative and $F_{\mu \nu}=\partial_{\mu} A_{\nu}-\partial_{\nu} A_{\mu}$ is the field tensor. This is invariant under local $\mathrm{U}(1)$ transformations $\phi \rightarrow e^{-i \alpha(x)} \phi$ when accompanied by a gauge transformation of the potential $A_{\mu} \rightarrow A_{\mu}+\frac{1}{q} \partial_{\mu} \alpha(x)$.

We are free to choose the unitary gauge $\alpha(x)=-\theta(x) / v$, absorbing the $\theta$-mode into the photon field $A_{\mu} \rightarrow A_{\mu}-\frac{1}{q v} \partial_{\mu} \theta(x)$. Ultimately, the final result is gauge-independent, but other choices require the Nambu-Goldstone bosons to be explicitly included in the 
Feynman rules. Since the $\theta$-mode is 'gauged away', excitations about the vacuum become

$$
\phi(x)=\frac{1}{\sqrt{2}}[v+h(x)]
$$

and the Lagrangian (1.10) becomes

$$
\mathcal{L}=\frac{1}{2} q^{2} v^{2} A_{\mu} A^{\mu}-\frac{1}{4} F_{\mu \nu} F^{\mu \nu}+\frac{1}{2} \partial_{\mu} h \partial^{\mu} h-\mu^{2} h^{2}+\ldots
$$

where the dots denote terms neither kinetic nor mass. The Nambu-Goldstone boson is no longer present and the photon has acquired a mass qv. Again, there is a massive scalar Higgs boson as a by-product of the SSB.

\subsubsection{Glashow-Salam-Weinberg electroweak theory}

The Higgs mechanism can be extended to non-abelian gauge theories, as was necessary to describe electroweak symmetry breaking $[22,23,36]$. Consider the Lagrangian for an $\mathrm{SU}(2) \times \mathrm{U}(1)$ gauge theory with a sombrero potential

$$
\mathcal{L}=\left(D_{\mu} \phi\right)^{\dagger}\left(D^{\mu} \phi\right)-\frac{1}{4} \boldsymbol{F}_{\mu \nu} \cdot \boldsymbol{F}^{\mu \nu}-\frac{1}{4} G_{\mu \nu} G^{\mu \nu}+\mu^{2} \phi^{\dagger} \phi-\lambda\left(\phi^{\dagger} \phi\right)^{2}
$$

where $D_{\mu}=\partial_{\mu}+\frac{i}{2} g \boldsymbol{\tau} \cdot \boldsymbol{W}_{\mu}+\frac{i}{2} g^{\prime} Y B_{\mu}$ is the covariant derivative, and $\boldsymbol{F}_{\mu \nu}=\partial_{\mu} \boldsymbol{W}_{\nu}-$ $\partial_{\nu} \boldsymbol{W}_{\mu}-g \boldsymbol{W}_{\mu} \times \boldsymbol{W}_{\nu}$ and $G_{\mu \nu}=\partial_{\mu} B_{\nu}-\partial_{\nu} B_{\mu}$ are the field tensors. In this case, $\phi$ is an $\mathrm{SU}(2)$ doublet of complex scalar fields

$$
\phi=\left(\begin{array}{c}
\phi^{+} \\
\phi^{0}
\end{array}\right)=\frac{1}{\sqrt{2}}\left(\begin{array}{c}
\phi_{1}+i \phi_{2} \\
\phi_{3}+i \phi_{4}
\end{array}\right) .
$$

Again, there are infinite degenerate vacua satisfying $\left(\phi_{1}^{2}+\phi_{2}^{2}+\phi_{3}^{2}+\phi_{4}^{2}\right)=\mu^{2} / \lambda$. In analogue with the abelian Higgs mechanism, the unitary gauge absorbs the $\phi_{1}, \phi_{2}$ and $\phi_{4}$-modes into the gauge fields. Thus, considering excitations about the vacuum

$$
\phi(x)=\frac{1}{\sqrt{2}}\left(\begin{array}{c}
0 \\
v+h(x)
\end{array}\right)
$$


the Lagrangian (1.13) becomes

$$
\begin{aligned}
\mathcal{L}=\frac{1}{8} & g^{2} v^{2} \boldsymbol{W}_{\mu} \cdot \boldsymbol{W}^{\mu}-\frac{1}{4} \boldsymbol{F}_{\mu \nu} \cdot \boldsymbol{F}^{\mu \nu}+\frac{1}{8} v^{2} g^{\prime 2} B_{\mu} B^{\mu}-\frac{1}{4} v^{2} g g^{\prime} B_{\mu} W_{3}^{\mu}-\frac{1}{4} G_{\mu \nu} G^{\mu \nu} \\
& +\frac{1}{2} \partial_{\mu} h \partial^{\mu} h-\mu^{2} h^{2}+\ldots \\
= & \frac{1}{4} g^{2} v^{2} W_{\mu}^{+} W^{-\mu}-\frac{1}{2}\left(\partial_{\mu} W_{\nu}^{+}-\partial_{\nu} W_{\mu}^{+}\right)\left(\partial^{\mu} W^{-\nu}-\partial^{\nu} W^{-\mu}\right) \\
& +\frac{1}{8} v^{2}\left(g^{2}+g^{\prime 2}\right) Z_{\mu} Z^{\mu}-\frac{1}{4}\left(\partial_{\mu} Z_{\nu}-\partial_{\nu} Z_{\mu}\right)\left(\partial^{\mu} Z^{\nu}-\partial^{\nu} Z^{\mu}\right)-\frac{1}{4} F_{\mu \nu} F^{\mu \nu} \\
& +\frac{1}{2} \partial_{\mu} h \partial^{\mu} h-\mu^{2} h^{2}+\ldots
\end{aligned}
$$

where the dots denote terms neither kinetic nor mass, $F_{\mu \nu}$ is the field tensor of QED, and the expression has been rewritten in terms of the physical gauge fields using (1.1), (1.2) and (1.3). The $W^{ \pm}$bosons acquire a mass $g v / 2$ and the $Z$ boson acquires a mass $v \sqrt{\left(g^{2}+g^{\prime 2}\right)} / 2$, while the photon is massless. Again, all Nambu-Goldstone bosons are gone and a Higgs boson has appeared as a by-product of the SSB.

This theory predicts a striking relation between the gauge boson masses, using (1.4)

$$
m_{W}=m_{Z} \cos \theta_{\mathrm{W}}
$$

which was experimentally verified once the $W$ and $Z$ bosons were discovered [37-41]. It also predicted a massive scalar Higgs boson, whose mass could not be determined from the other parameters of the theory.

Fermion masses can also be incorporated into EW theory through Yukawa couplings. Consider a coupling between the electron-type $\mathrm{SU}(2)$ doublet (see Table 1.1), the Higgs doublet $\phi$ given in (1.15), and the electron $\mathrm{SU}(2)$ singlet

$$
\begin{aligned}
\mathcal{L}_{e}^{\mathrm{Yuk}} & =-g_{e}\left(\bar{\ell}_{e \mathrm{~L}} \phi e_{\mathrm{R}}+\bar{e}_{\mathrm{R}} \phi^{\dagger} \ell_{e \mathrm{~L}}\right) \\
& =-\frac{g_{e}}{\sqrt{2}}[v+h]\left(\bar{e}_{\mathrm{L}} e_{\mathrm{R}}+\bar{e}_{\mathrm{R}} e_{\mathrm{L}}\right)
\end{aligned}
$$

where $g_{e}$ is the electron Yukawa coupling. The electron has acquired a mass $g_{e} v / \sqrt{2}$ and the coupling of the Higgs boson to the electron is proportional to that mass (specifically $\left.m_{e} / v\right)$.

Finally, we note a similar phenomenon in superconductors. There, the U(1) symmetry of QED is spontaneously broken, as in Section 1.2.2, giving mass to the photon and thereby producing the Meissner effect. In fact, Higgs bosons have been observed in the Raman spectra of superconductors [42]. However, a major difference is that the bosonic 
field is a Bose-Einstein condensate of loosely bound electron pairs (known as Cooper pairs), and therefore the SSB is dynamic. This is only possible due to lattice vibrations of the underlying solid. It is natural to ask whether a similar dynamic mechanism could be used to break EW symmetry, where the Higgs boson is a composite particle. This is an active area of research, though will not be explored here.

\subsection{Properties of the Higgs boson}

The Higgs boson is predicted to have zero spin and positive parity, whilst being electrically neutral and colourless. It couples directly to massive particles. Other properties, such as production cross sections and branching ratios (BRs) of decay, must be calculated as a function of its mass, which is not predicted by the SM.

At a hadron collider such as the LHC, the important production modes are gluongluon fusion ( $g g F)$, vector boson fusion (VBF), Higgs-strahlung ( $W H$ and $Z H$ ) and top fusion $(t t H)$. Example Feynman diagrams are shown in Figure 1.3. We note that the

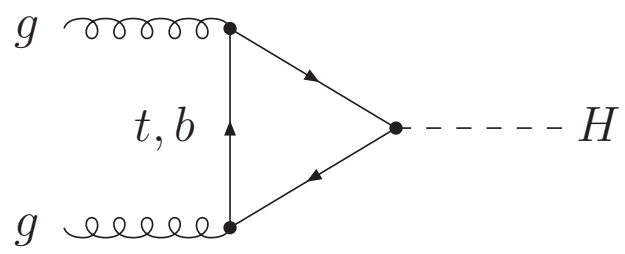

(a) Gluon-gluon fusion (ggF)

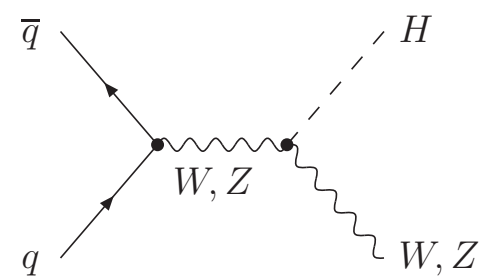

(c) Higgs-strahlung $(W H$ and $Z H)$

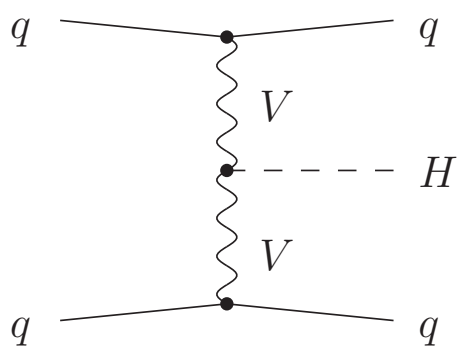

(b) Vector boson fusion (VBF)

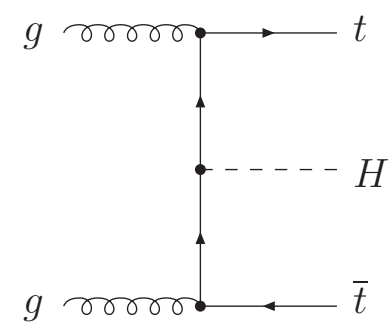

(d) Top fusion $(t t H)$

Figure 1.3: Examples of tree-level Feynman diagrams for the Higgs production processes relevant at the LHC. 

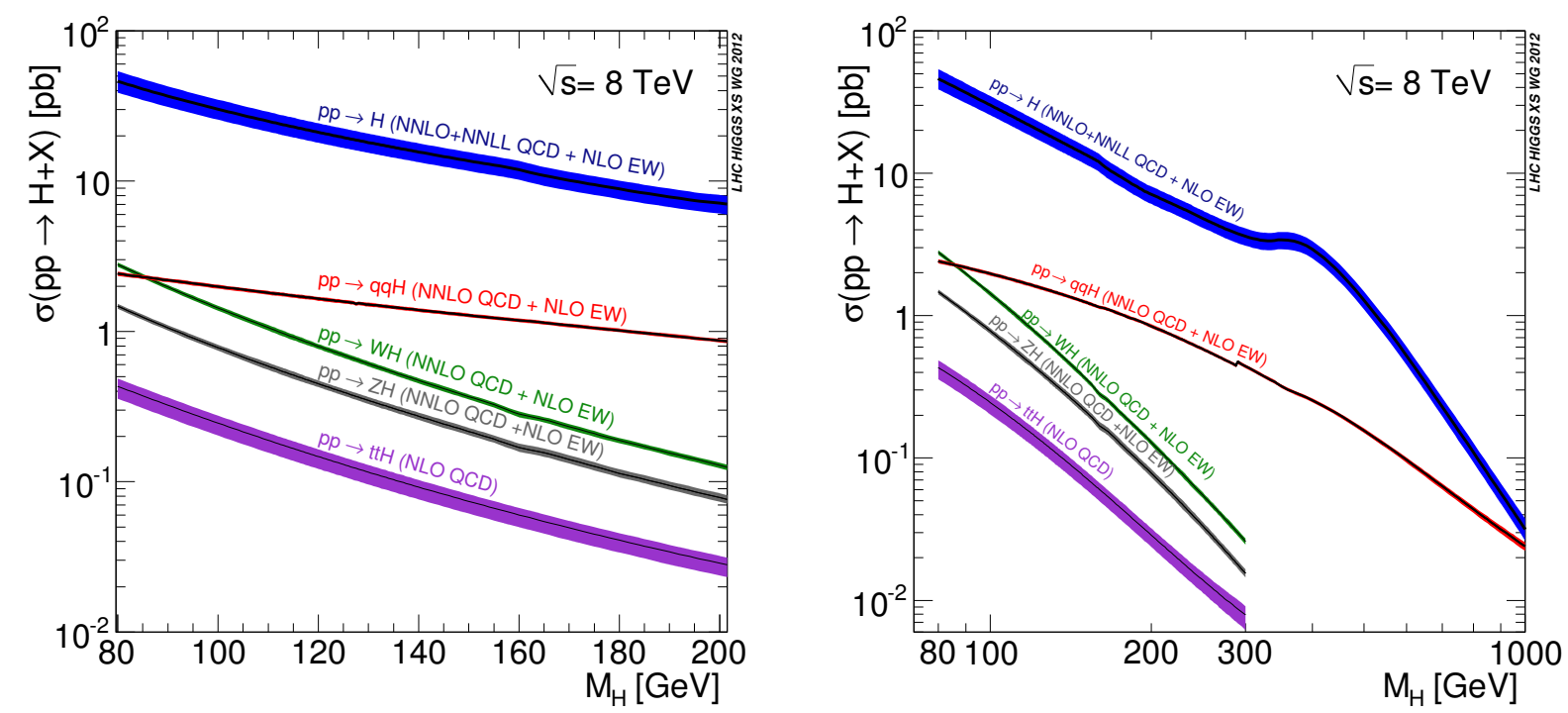

Figure 1.4: Higgs boson production cross sections versus mass at $\sqrt{s}=8 \mathrm{TeV}$ for a low mass range (left) and an expanded mass range (right) [43]. Theoretical uncertainties are shown as bands. The production modes are ggF (blue), VBF (red), $W H$ (green), $Z H$ (grey) and $t t H$ (purple).

Higgs boson does not couple to massless gluons, therefore ggF proceeds via loops of massive coloured particles (predominantly the top quark due to its large mass).

The production cross sections at the LHC are shown in Figure 1.4. Whilst ggF clearly dominates these rare processes, it suffers from large experimental backgrounds. The four other modes feature additional final state particles which can aid identification. For example, VBF has two well-separated quarks with no colour exchange between them.

Since the lifetime of the Higgs boson is very short, it is never directly observed in a detector. Therefore it is important to understand the BRs of its decays (Figure 1.5). Naïvely, these are understood from the Higgs boson coupling to mass and the kinematic requirement $m_{H}>m_{X}+m_{Y}$ for a decay $H \rightarrow X Y$. This is complicated by off-shell particles (e.g. a low mass Higgs boson may decay to $W W^{*}$ ). Also, the $\gamma \gamma, Z \gamma$ and $g g$ decay modes are different since they feature massless particles, and therefore proceed via loops of massive charged particles (electric charge for $\gamma \gamma$ and $Z \gamma$, colour charge for $g g$ ).

Designing a sensitive experimental search strategy for the Higgs boson can be difficult. In decay channels featuring weak bosons, the subsequent decay of the $W$ or $Z$ boson must also be considered. These are more likely to decay to quarks than to leptons, but the former suffers from large backgrounds at hadron colliders. Similarly, the $b \bar{b}$ decay has the largest BR for low $m_{H}$ but suffers from huge backgrounds. The sensitivity can 

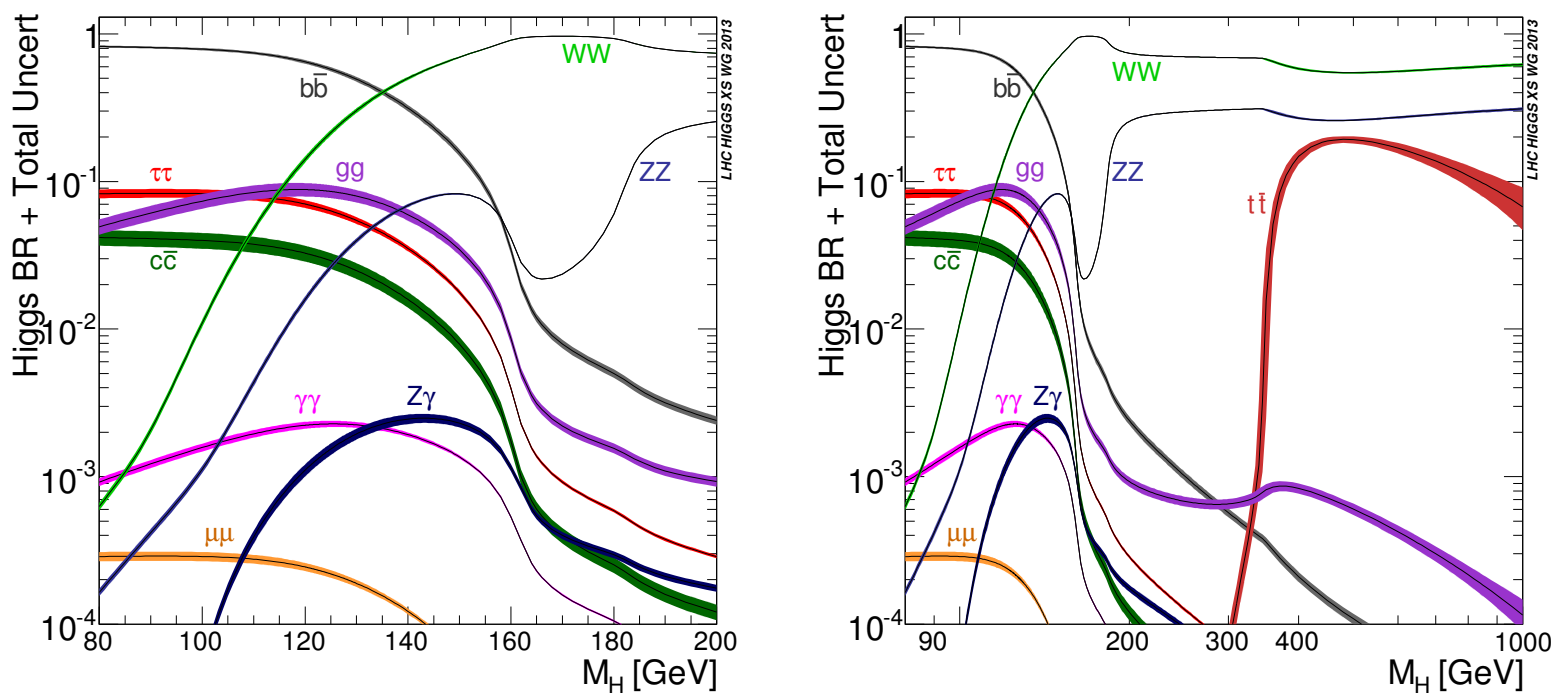

Figure 1.5: Branching ratios of Higgs boson decay versus mass for a low mass range (left) and an expanded mass range (right) [10]. Theoretical uncertainties are shown as bands.

be improved by combining with a more distinguished production mode, such as $W H$ or $Z H$, but this reduces the production cross section.

\subsection{Pre-LHC constraints on the Higgs boson mass}

Neglecting Yukawa interactions, the EW sector of the SM contains several free parameters that must be experimentally determined: two couplings $\left(g, g^{\prime}\right)$ and two Higgs sector parameters $(\mu, \lambda)$. Using relations in Section 1.2, it is advantageous to choose an alternative set of independent parameters more closely connected to experiment: $\alpha_{\mathrm{EM}}$, $m_{W}, m_{Z}, m_{H}$. Finding the Higgs boson and measuring its mass is therefore of fundamental importance to understanding the EW sector, and this was a primary goal of the LHC physics program.

Prior to the LHC, the value of $m_{H}$ was constrained through direct searches at previous colliders, global fits of other electroweak observables and theoretical considerations. 


\subsubsection{Direct searches}

Although masses below $4 \mathrm{GeV}$ were excluded from $B, \Upsilon$ and $K$ meson decays [44], the first meaningful searches for a Higgs boson were performed at LEP (CERN, Geneva), which ran from 1989 to 2000. This was a circular $e^{+} e^{-}$collider with a centre-of-mass (CM) energy tuned to the $Z$-pole and then later varied between 189 and $209 \mathrm{GeV}$. A combined search for $Z H$ was performed using a total integrated luminosity of $2.5 \mathrm{fb}^{-1}$, which excluded $m_{H}<114.4 \mathrm{GeV}$ at the $95 \%$ confidence level (CL) [45].

Further searches were performed at the Tevatron (FNAL, Illinois), which ran from 1987 to 2011. This was a circular $p \bar{p}$ collider with $\mathrm{CM}$ energies of $1.8 \mathrm{TeV}$ and $1.96 \mathrm{TeV}$. In 2010, searches using a variety of production and decay modes were combined across experiments using a total integrated luminosity of up to $12.6 \mathrm{fb}^{-1}$. Masses below $109 \mathrm{GeV}$ and between 158 and $175 \mathrm{GeV}$ were excluded at the 95\% CL [46].

\subsubsection{Precision electroweak fits}

The SM predicts that many observables will depend upon $m_{H}$ through loop corrections, and it is therefore possible to infer $m_{H}$ through precision EW measurements. Since the leading $m_{H}$ dependence is logarithmic, the inferred constraints are weaker than those used to predict the top mass (where the dependence is quadratic).

Performing a global fit of various electroweak measurements at the LEP, SLC and Tevatron colliders $\left(\right.$ e.g. $\left.m_{W}, m_{Z}, m_{t}\right)$, in July 2010 it was possible to exclude $m_{H}>$ $158 \mathrm{GeV}$ at the $95 \% \mathrm{CL}$ [47]. However, the best fit value was excluded by direct searches, as shown in Figure 1.6.

\subsubsection{Theoretical constraints}

Like all coupling constants in a renormalisable theory (see Section 2.1.1), the Higgs quartic coupling $\lambda$ 'runs' with energy scale $\Lambda$, as described by the renormalisation group equations (RGEs). The running is characterised by the $\beta$-function:

$$
\beta_{\lambda}=\frac{\partial \lambda}{\partial \log \Lambda}
$$



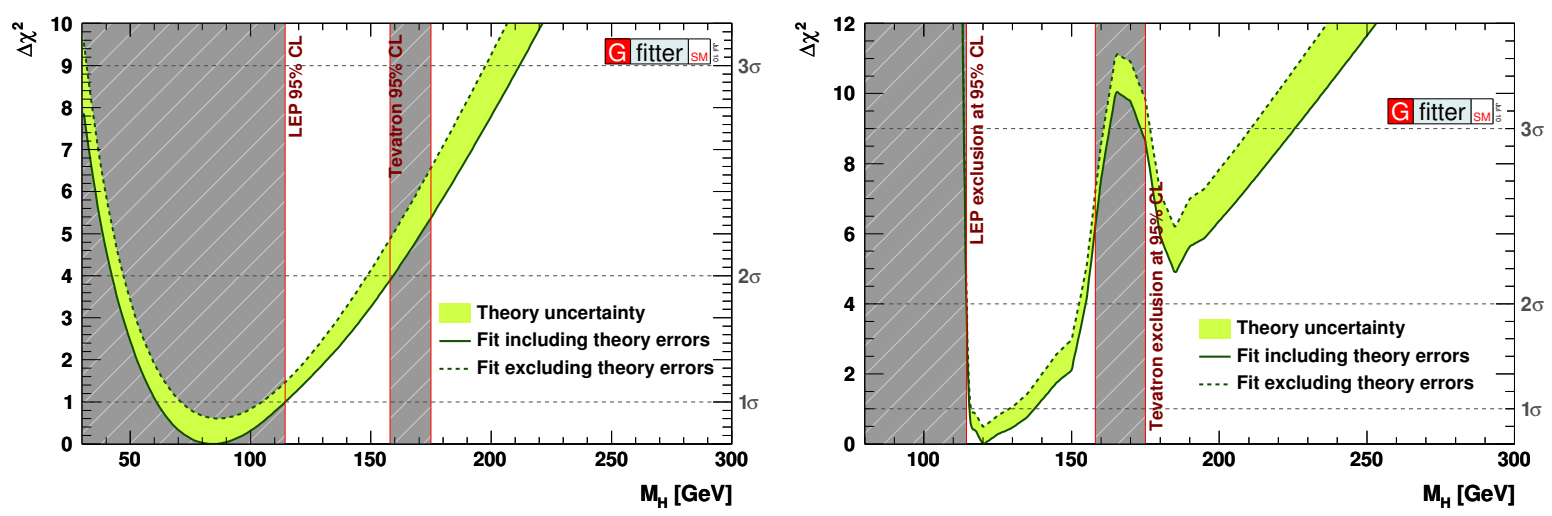

Figure 1.6: The observed $\Delta \chi^{2}=\chi^{2}-\chi_{\min }^{2}$ of electroweak fits versus $m_{H}$, neglecting (left) and including (right) results from direct searches [47]. The exclusion limits from LEP and the Tevatron are also shown. These results were produced in July 2010.

For high $m_{H}$, self-couplings dominate $\beta_{\lambda}$, which have a positive contribution. Therefore $\lambda$ increases with the scale, and above some critical scale $\Lambda_{\mathrm{c}}$ the EW theory is no longer perturbative. Thus we would either expect to observe non-perturbative behaviour at scales $\sim \Lambda_{\text {c }}$ or new physics at a scale $<\Lambda_{\text {c }}$ that circumvents this issue. Larger values of $m_{H}$ lead to lower values of $\Lambda_{\mathrm{c}}$ and are therefore disfavoured (blue line in Figure 1.7). Requiring perturbativity up to the reduced Planck scale of $\bar{\Lambda}_{\mathrm{P}} \sim 10^{18} \mathrm{GeV}$ (where we expect new physics describing gravity) places an upper bound on $m_{H}$ of $175 \mathrm{GeV}$ [48].

For small $m_{H}$, top loops dominate $\beta_{\lambda}$, which have a negative contribution. Therefore $\lambda$ decreases as the scale increases, and above some critical scale $\Lambda_{\mathrm{c}}$ the coupling becomes negative. Then the EW vacuum is simply a local minimum and it is possible for the Universe to collapse through quantum tunnelling into the more stable vacuum state (yellow band in Figure 1.7). Requiring vacuum stability up to $\bar{\Lambda}_{\mathrm{P}}$ places a lower bound on $m_{H}$ of $129 \mathrm{GeV}$ [48]. It is also possible to consider a metastable Universe whose expected lifetime is longer than its age. Accounting for thermal fluctuations up to temperatures $\sim \bar{\Lambda}_{\mathrm{P}}$, the EW vacuum has a lifetime longer than the age of the Universe if $m_{H}>122 \mathrm{GeV}$ (pale blue band in Figure 1.7) [48]. These bounds are rather sensitive to the top mass, which is $m_{t}=173.34 \pm 0.27$ (stat) \pm 0.71 (syst) $\mathrm{GeV}[49]$. 


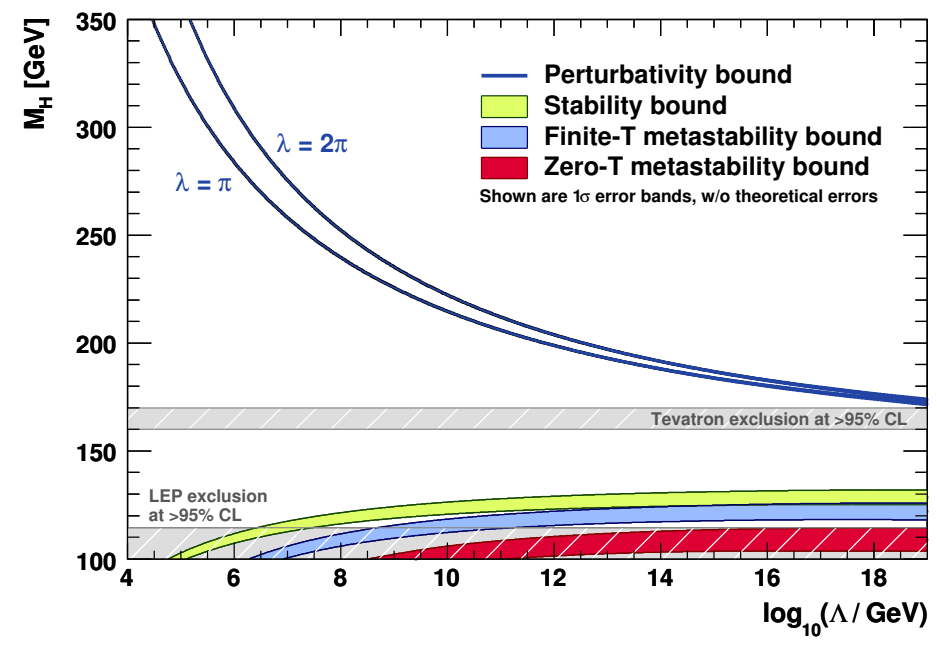

Figure 1.7: The scale $\Lambda$ at which the Higgs quartic coupling becomes non-perturbative (blue lines) or an instability in the EW vacuum appears (yellow band) [48]. The two blue lines represent different degrees of non-perturbativity (lower line corresponds to a two-loop correction of $25 \%$, upper line is $50 \%$ ), and their difference is indicative of the theoretical uncertainty in this bound. The blue and red bands are bounds for a metastable Universe including and neglecting thermal fluctuations respectively. Reprinted from Physics Letters B 679, 4, J. Ellis, J. R. Espinosa, G. F. Giudice, A. Hoecker and A. Riotto, The Probable Fate of the Standard Model, 369-375, Copyright (2009), with permission from Elsevier. 


\section{Chapter 2}

\section{Computational techniques for the LHC}

Although the search for the Higgs boson is motivated by the electroweak interaction, a detailed knowledge of quantum chromodynamics (QCD) is required to make precise predictions at a hadron collider such as the LHC. However, these calculations are troublesome; QCD describes the interactions of quarks and gluons, though only the composite hadrons are experimentally observed.

Some key concepts of QCD are introduced in Section 2.1, before the simulation of LHC collisions is described in Section 2.2. Finally, jets are introduced in Section 2.3 as useful tools connecting theoretical calculations with experimental observations.

\subsection{Quantum chromodynamics}

QCD is the theory of the strong interaction, describing coloured particles (quarks and gluons, collectively known as partons) [50]. Two crucial features of QCD are confinement and asymptotic freedom. Confinement refers to the observation that quarks and gluons are only found within colourless hadrons, and never as isolated states. Asymptotic freedom states that, within the hadron, the constituent partons are relatively free to move. Both concepts can be understood in terms of a running coupling constant.

\subsubsection{Renormalisation and the running coupling constant}

When calculating observables within perturbative quantum field theory, ultraviolet (UV) divergences are often introduced by Feynman diagrams containing loops. Through careful 


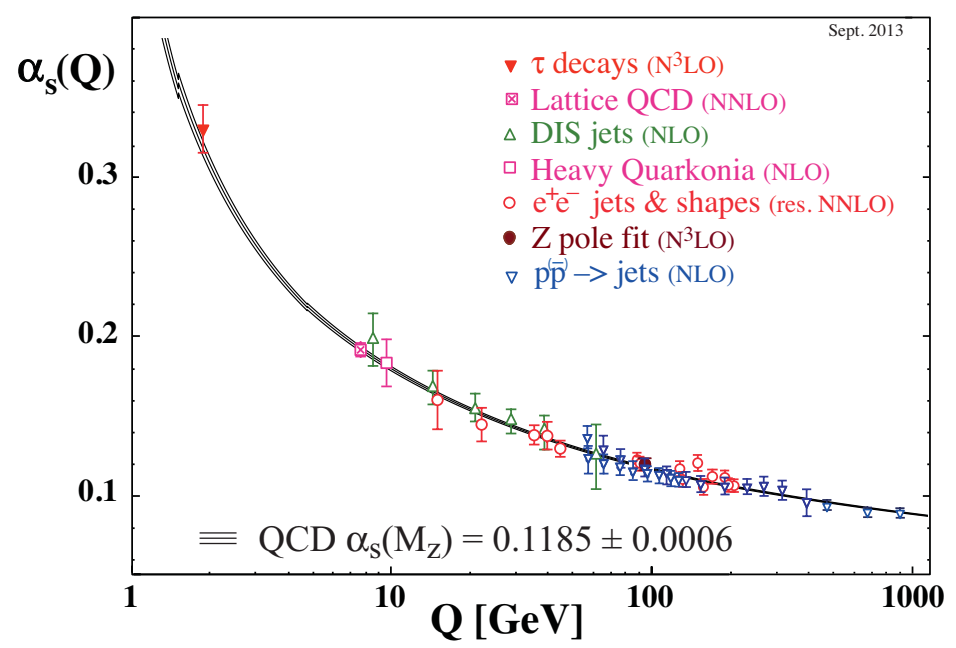

Figure 2.1: The running of the strong coupling constant $\alpha_{\mathrm{S}}$ with energy scale $Q$ [26]. Experimental measurements at various scales are also shown.

consideration, these UV divergences can be absorbed into renormalised definitions of the coupling constant and particle masses. The idea is that the 'bare' quantities contain compensating divergences, such that the physically measurable quantities are finite:

$$
g_{\text {physical }}=g_{\text {bare }}+\delta g \quad \text { and } \quad m_{\text {physical }}=m_{\text {bare }}+\delta m
$$

where $\delta g$ and $\delta m$ are the loop contributions. This procedure is known as renormalisation.

It is necessary to introduce an unphysical renormalisation scale $\mu_{\mathrm{R}}$, above which loops are absorbed into renormalised quantities, and below which loops are calculated in perturbation theory. The exact details of the separation depend upon the choice of renormalisation scheme [19]. Clearly couplings and masses will depend upon $\mu_{\mathrm{R}}$, though physical observables must not; however, truncation of the perturbative series will result in a residual $\mu_{\mathrm{R}}$ dependence. Usually $\mu_{\mathrm{R}}$ is chosen to be the energy scale $Q$ of the process under consideration, leading to the concept of a running coupling constant.

The QCD coupling constant $\alpha_{\mathrm{S}}$ is shown in Figure 2.1. At low scales (large distances), $\alpha_{\mathrm{S}}$ is large and the theory is non-perturbative. Though not analytically proven ${ }^{1}$, confinement has been verified in this regime by lattice QCD [51]. At high scales (small distances), $\alpha_{\mathrm{S}}$ is small; this is asymptotic freedom $[52,53]$. Note that $\alpha_{\mathrm{EM}}$ in QED exhibits an opposite trend, though remains perturbative at all accessible energies.

${ }^{1}$ A mathematically rigorous proof of confinement is one of seven Millennium Prize Problems of the Clay Mathematics Institute, with a bounty of $\$ 1,000,000$. 


\subsubsection{Perturbative QCD}

Most interesting LHC processes involve a large momentum transfer, where the partons are asymptotically free. Thus, parton-level cross sections may be calculated with Feynman diagrams as a perturbative series in $\alpha_{\mathrm{S}}$ (which converges since $\alpha_{\mathrm{S}} \ll 1$ )

$$
\hat{\sigma}=\sum_{m=0}^{\infty} \alpha_{\mathrm{S}}^{k+m} \hat{\sigma}^{(m)}
$$

where the hat denotes a parton-level quantity, $k$ is the number of QCD vertices at tree-level, and $\hat{\sigma}^{(m)}$ is the $m$ th order contribution to the cross section. A fixed order calculation truncates the series after $n$ terms, with $n=0$ being a leading order (LO) calculation, $n=1$ being a next-to-leading order (NLO) calculation, and so on.

As mentioned in Section 2.1.1, the cross section $\hat{\sigma}$ is independent of the renormalisation scale $\mu_{\mathrm{R}}$

$$
\frac{\mathrm{d} \hat{\sigma}}{\mathrm{d} \mu_{\mathrm{R}}}=0
$$

However, real-life calculations always truncate the series after $n$ terms, leaving a residual $\mu_{\mathrm{R}}$ dependence. Inserting the truncated series into (2.3), it follows that

$$
\begin{aligned}
\frac{\mathrm{d}}{\mathrm{d} \mu_{\mathrm{R}}} \sum_{m=0}^{n} \alpha_{\mathrm{S}}^{k+m} \hat{\sigma}^{(m)} & =-\frac{\mathrm{d}}{\mathrm{d} \mu_{\mathrm{R}}} \sum_{m=n+1}^{\infty} \alpha_{\mathrm{S}}^{k+m} \hat{\sigma}^{(m)} \\
& =\mathcal{O}\left(\alpha_{\mathrm{S}}^{k+n+1}\right) .
\end{aligned}
$$

Thus, the residual $\mu_{\mathrm{R}}$ dependence can be exploited to probe the effect of missing higher order terms in the series, and estimate the associated uncertainty.

\subsubsection{Resummation of large logarithms}

Fixed order calculations are useful only when the perturbative series is converging, as is usual for an inclusive cross section. However, when considering exclusive observables, there are regions of phase space in which the missing higher order terms contribute as much as the included terms. This often occurs when there is a large separation in the scales of the exclusive observables and the process. 
For example, consider the emission of a gluon from an outgoing quark. The scale separation of the hard scatter $Q$ from the soft emission $Q_{1}$ introduces Sudakov double logarithmic contributions $\alpha_{\mathrm{S}}^{k+m} L^{2 m}$ to the perturbative series, where $L \sim \ln \left(Q_{1} / Q\right)$. Requiring such an emission, the (schematic) structure of the perturbative series becomes

$$
\hat{\sigma} \sim \alpha_{\mathrm{S}}^{k}\left\{\alpha_{\mathrm{S}}\left(L^{2}+L+1\right)+\alpha_{\mathrm{S}}^{2}\left(L^{4}+L^{3}+L^{2}+L+1\right)+\mathcal{O}\left(\alpha_{\mathrm{S}}^{3} L^{6}\right)\right\}
$$

Soft or collinear emissions are defined by $\alpha_{\mathrm{S}} L^{2} \approx 1$, such that the logarithms overcome the $\alpha_{\mathrm{S}}$ suppression. Thus, the perturbative nature of the series is spoiled. In (2.6), terms like $\alpha_{\mathrm{S}}^{k+m} L^{2 m}$ are called leading logarithms (LLs), terms like $\alpha_{\mathrm{S}}^{k+m} L^{2 m-1}$ are called next-to-leading logarithms (NLLs), and so on.

When an observable is sensitive to such large logarithms, they must be resummed to all orders in $\alpha_{\mathrm{S}}$ to produce an accurate result. This is usually achieved analytically, but in this example of soft and collinear emissions a parton shower Monte Carlo program can be used. This probabilistically generates emissions as it evolves partons from the scale of the hard scatter down to a scale where non-perturbative effects of confinement dominate. This leads to fully-exclusive observables. A parton shower is necessary to produce hadron-level predictions (see Section 2.2). Formally they have LL accuracy, though can include many higher order logarithms through the enforcement of physical effects such as energy-momentum conservation and colour coherence.

\subsubsection{Parton distribution functions}

Since confinement binds partons into hadrons, it is the latter that are accelerated and collided at the LHC (in particular protons). Therefore, we need to calculate observables for proton-proton interactions rather than the parton-parton interactions discussed above. Fortunately, the factorisation theorem states that the soft non-perturbative physics of the hadron can be treated independently of the hard scatter [54]. Thus, a proton-proton cross section can be formulated as a convolution of the partonic cross section with parton distribution functions (PDFs) of the incoming protons. That is,

$$
\sigma\left(p_{1}, p_{2}\right)=\sum_{a, b} \int_{0}^{1} \mathrm{~d} x_{1} \mathrm{~d} x_{2} f_{a}\left(x_{1}, \mu_{\mathrm{F}}^{2}\right) f_{b}\left(x_{2}, \mu_{\mathrm{F}}^{2}\right) \hat{\sigma}_{a b}\left(x_{1} p_{1}, x_{2} p_{2}, \alpha_{\mathrm{S}}\left(\mu_{\mathrm{R}}^{2}\right), \frac{Q^{2}}{\mu_{\mathrm{F}}^{2}}, \frac{Q^{2}}{\mu_{\mathrm{R}}^{2}}\right)
$$


MSTW 2008 NLO PDFs (68\% C.L.)
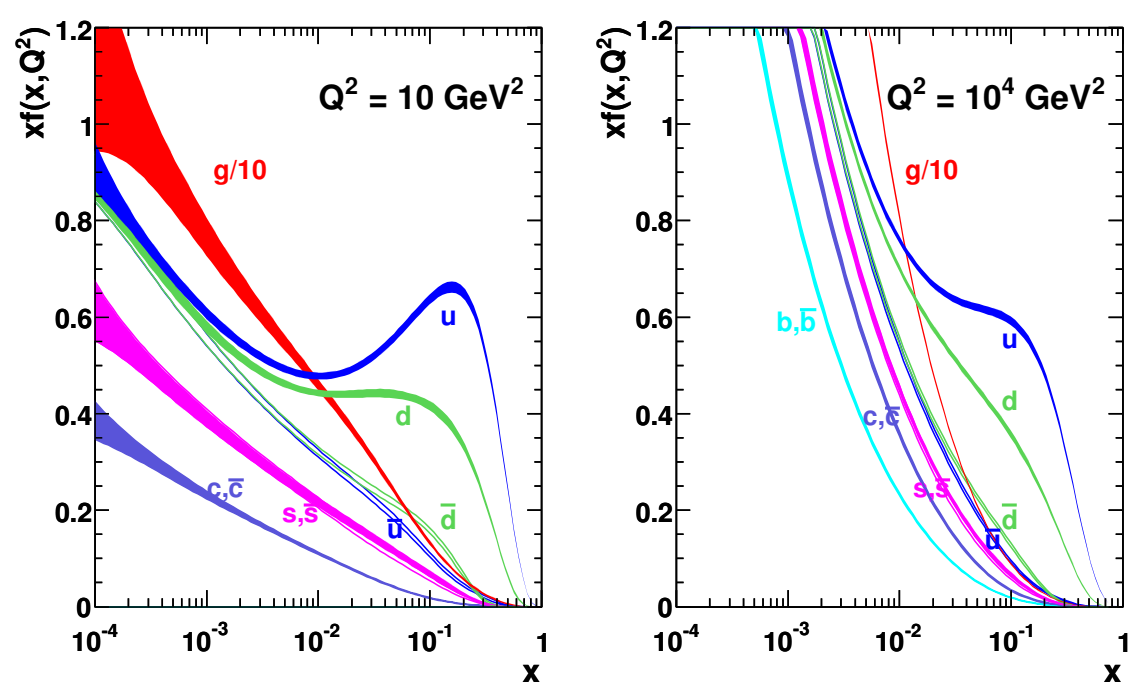

Figure 2.2: Parton distribution functions fit by the MSTW collaboration, evaluated at $\mu_{\mathrm{F}}^{2}=10 \mathrm{GeV}^{2}$ (left) and $\mu_{\mathrm{F}}^{2}=10^{4} \mathrm{GeV}^{2}$ (right) [58]. Note that the gluon PDF is suppressed by a factor 10. Reprinted with kind permission from Springer Science and Business Media: European Physical Journal C, 63, 2009, 189-285, Parton distributions for the LHC, A. D. Martin, W. J. Stirling, R. S. Thorne and G. Watt, Figure 1, Copyright 2009. MSTW 2008 NLO PDFs at $Q^{2}=10 \mathrm{GeV}^{2}$ and $Q^{2}=10^{4} \mathrm{GeV}^{2}$.

where $f_{a}$ is the PDF of parton type $a$ within the proton, $p_{i}$ is the momentum of proton $i$, $x_{i}$ is the momentum fraction of parton $i$, and $Q$ is the scale of the hard scatter. A sum is performed over all possible parton types (six quark flavours and the gluon).

Echoing renormalisation, factorisation absorbs collinear divergences into universal PDFs which are not a priori calculable and must be experimentally constrained. An unphysical factorisation scale $\mu_{\mathrm{F}}$ is introduced, below which emissions are absorbed into PDFs, and above which they are included in the hard scatter. As with $\mu_{\mathrm{R}}$, truncating the perturbative series introduces a $\mu_{\mathrm{F}}$ dependence, which can be exploited to estimate the effect of the missing higher order terms. At LO, $f_{a}\left(x, \mu_{\mathrm{F}}\right)$ is simply the probability of finding a parton of type $a$ with momentum fraction $x$, when probing the proton at a scale $\mu_{\mathrm{F}}$. However, the interpretation at higher orders is more complicated.

The PDF $\mu_{\mathrm{F}}$ scaling is described by the DGLAP equations [55-57]. Thus, an $f_{a}(x)$ ansatz is made at low $\mu_{\mathrm{F}}$ and then experimentally validated at higher scales (e.g. with deep inelastic scattering or collider jet data). Figure 2.2 shows some example PDFs. 


\subsection{Monte Carlo event generation}

Monte Carlo (MC) event generators provide a fully-exclusive hadron-level simulation of $p p$ collision events at the LHC [59]. This section will describe the basic features of a simulated event, before discussing some more advanced techniques that shall be used throughout the thesis.

\subsubsection{The anatomy of an event}

Figure 2.3 shows how the MC event generation is factorised into several components, each describing a certain regime of momentum transfer.

\section{Hard scatter}

The high scale process can be selected as desired (e.g. Higgs boson production via gluon-gluon fusion). The relevant parton-level matrix elements (MEs) are calculated using fixed order perturbative QCD, either by the event generator itself or an external program. Historically, these MEs were usually LO, though improvements are discussed in Sections 2.2.3 and 2.2.4.

\section{Parton distribution functions (PDFs)}

Incoming parton momenta are sampled from a proton PDF, usually probed at the scale of the hard scatter $\left(\mu_{\mathrm{F}}=Q\right)$. The LHAPDF interface [61] provides access to the PDFs of several fitting collaborations, such as CTEQ [62], MSTW [58] and NNPDF [63]. PDFs differ because they are fit with different subsets of experimental data, massive quark treatments, parametrisation models and $\alpha_{\mathrm{S}}\left(m_{Z}\right)$ values.

\section{Final state radiation (FSR)}

Soft and collinear radiation from outgoing partons is simulated by a universal parton shower, evolving the scale from the hard scatter to the hadronisation scale of $\sim 1 \mathrm{GeV}$. The successive emissions are ordered to avoid double-counting - typical order parameters are virtuality, transverse momentum and opening angle.

For the correct treatment of soft emissions, it is vital to preserve colour coherence. This is inherent in an angular ordered shower, but must be manually implemented otherwise. Alternatively, a dipole shower considers emissions from colour-connected pairs of partons, and is also inherently coherent. 


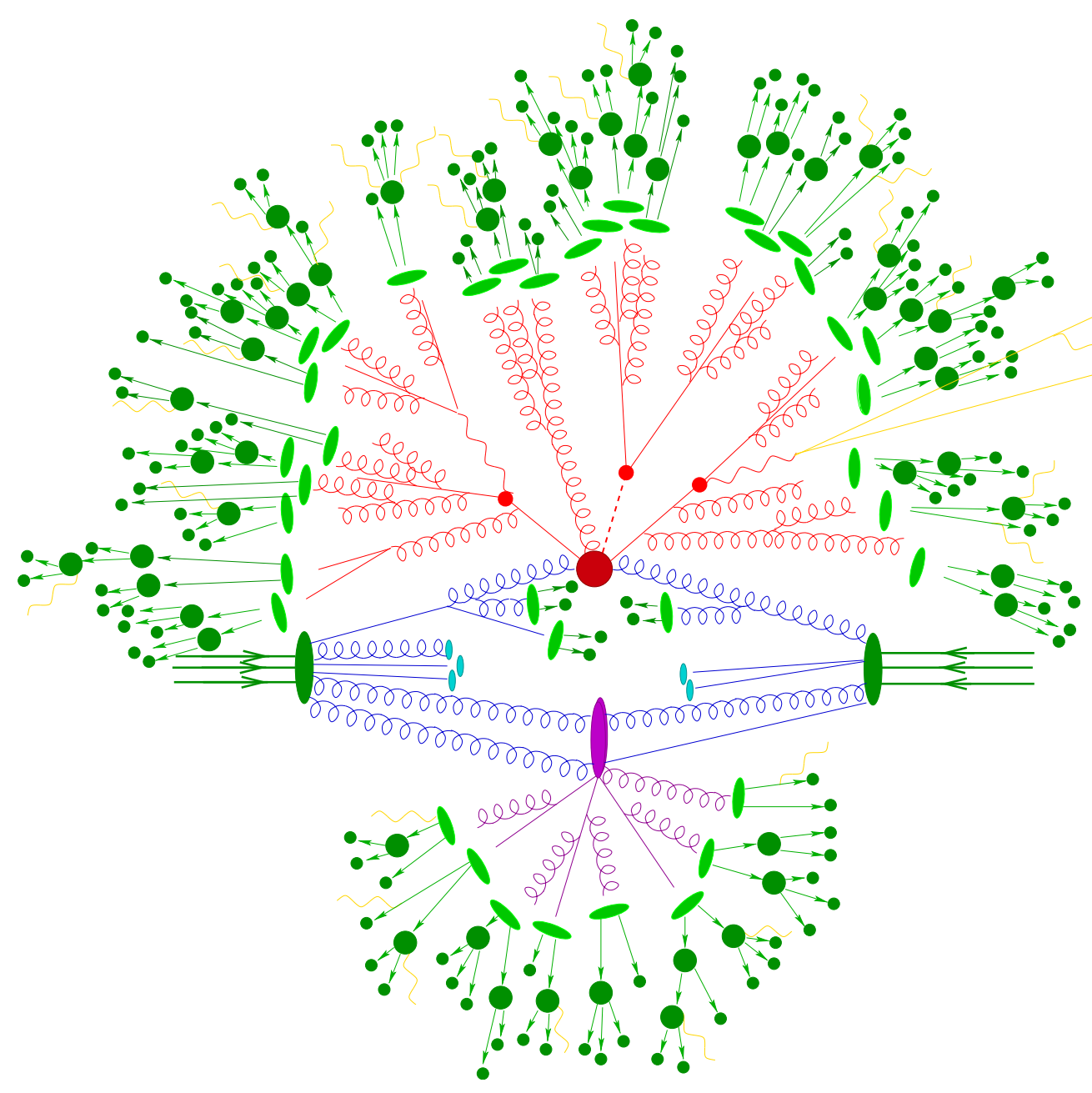

Figure 2.3: Schematic diagram of a simulated $t t H$ event, showing how factorisation allows the physics at different scales of momentum transfer $Q$ to be treated independently [60]. At high- $Q$ is the hard scatter (red circle). As the scale evolves down, partons are radiated in the initial state (blue) and final state (red). At low- $Q$, incoming partons are confined to the beam protons, while outgoing partons hadronise (green blobs). The underlying event comprises multiple partonic interactions (purple blob) and beam remnants (blue blobs). Photons and leptons (yellow) are also radiated. 


\section{Initial state radiation (ISR)}

Soft and collinear radiation from incoming partons is similarly described by a parton shower. However, the small probability of evolving two partons with the kinematics required by the hard process necessitates a backwards evolution. Thus, the probability that a parton originated from one of higher momentum and lower scale is calculated, rather than an emission probability.

\section{Hadronisation}

The confinement of partons to hadrons is non-perturbative, and must be described by a hadronisation model. The string model stretches strings between colour partners. At some distance it becomes favourable to convert the potential energy to a $q \bar{q}$ pair, breaking the string. Once there is insufficient energy to create $q \bar{q}$ pairs, the hadrons 'freeze out'. The cluster model splits gluons into $q \bar{q}$ pairs, which group into colourless clusters with a mass spectrum predicted by QCD. These clusters then decay to the physical hadrons. Note that all hadronisation models require tuning to experimental data.

\section{Hadron and $\tau$ decays}

Many of the hadrons produced during hadronisation are unstable, and must be decayed to particles that are stable on a detector traversal timescale, while observing conservation laws and measured branching ratios. Similarly, the $\tau$ lepton must be decayed, hadronically or leptonically.

\section{Multiple partonic interactions (MPI)}

The underlying event (UE) is the additional soft hadronic activity caused by partons inactive in the hard scatter. It comprises the breakup of the beam remnants and multiple partonic interactions (MPI) between the protons. The size of the MPI activity is correlated to the scale of the hard scatter.

In order to calculate the number of additional interactions, the spatial distribution of partons within the proton must be modelled, the impact parameter of the $p p$ collision must be known, and an IR cut-off must be imposed. This requires non-perturbative models that must be tuned to experimental data.

\section{QED radiation}

Electrically charged particles can emit photons at any stage of the event generation. 


\subsubsection{Summary of event generators}

Three event generators are commonly used at the LHC, mainly differing in their choice of hadronisation and MPI models, and their parton shower order parameter. Efforts to rewrite the older Fortran-based programs in C++ have led to a generation of 'out-of-date' Fortran programs that are no longer actively developed. Even so, they are still in common usage, and so are included in the descriptions below.

\section{Herwig}

Herwig (Fortran) [64] and HerwiG++ (C++) [65] both employ an angular ordered parton shower and a cluster hadronisation model. An MPI model is included in Herwig++, but in HeRWIG this was provided by JimmY [66].

\section{Pythia}

Pythia 6 (Fortran) [67] and Pyтhia 8 (C++) [68] both use a string hadronisation model and an advanced MPI model. PYTHIA 8 uses a dipole shower ordered in transverse momentum, whereas PYTHIA 6 offers a choice of virtuality or transverse momentum ordered parton showers with colour coherence implemented manually.

\section{Sherpa}

Sherpa (C++) [69] uses a dipole shower ordered in transverse momentum, which is convenient for multi-leg merging (see Section 2.2.3). It uses a cluster hadronisation model and an MPI model similar to that of PYTHIA 8.

\subsubsection{Multi-leg merging}

Although a parton shower excellently describes the emissions of large numbers of soft and collinear partons, it fails to accurately model hard and isolated emissions. It can be desirable to describe these using fixed order MEs, which are better suited to the task. In doing so, a couple of immediate issues arise. First, we require a smooth transition from the emissions of an ME to those of the parton shower. Second, each ME is inclusive, and attempting to combine MEs of differing multiplicity naturally leads to problems of double counting.

By using a merging prescription, such as the CKKW-L algorithm [70,71] employed by Sherpa or the MLM algorithm [72] employed by Alpgen [73] and MAdGraph [74], it is possible to consistently combine LO matrix elements with differing multiplicities, whilst matching to the parton shower correctly. This does require the introduction of a 
merging scale though. This scale separates the ME and parton shower descriptions of the emissions, though the details of the separation depend upon the merging prescription.

\subsubsection{NLO matching}

It is also possible to match an NLO ME to a parton shower, to improve the accuracy of both the normalisation and distribution of observables [75]. Such a calculation must include the LO, virtual-loop and real-emission diagrams, while mapping smoothly onto the parton shower for soft emissions. There are currently two valid matching prescriptions:

\section{MCONLO}

Simply adding a parton shower to an NLO ME introduces double counting of emissions. The MC@NLO method compensates for this overlap through a correction to the NLO calculation. This correction renders the ME dependent upon the parton shower used in the MC event generator, and is also a source of negatively weighted events.

Originally implemented in the MC@NLO program for matching to HeRwIG [76] and HeRwiG++ [77], the method has now been automated within the AMC@NLO program [78] and extended for use with Pyтнia 6 and Pythia 8 [79]. It is now also included in SHERPA.

\section{POWHEG}

The Powheg method requires the hardest emission to always be generated by the ME. It achieves the correct hard and soft behaviour by convolving the LO ME with a modified Sudakov factor, and then reweighting the differential cross section to the NLO result. Thus the ME is independent of the subsequent parton shower. However, if the parton shower is not transverse momentum ordered, it is necessary to use truncated and vetoed parton showers to correctly fill the phase space.

Originally implemented in PowhegBox [80-82], variants are now also included in HERWIG++ and SHERPA. 


\subsubsection{Additional considerations}

\section{Detector simulation}

In order to compare MC events to experimental events recorded at the LHC, it is vital to simulate how the outgoing particles interact with the detector. This is also necessary to calibrate the detector response and estimate efficiencies. GEANT4 $[83,84]$ is used to simulate the energy deposition of each particle during its trajectory through the ATLAS detector (see Chapter 3). Since long-lived particles will decay en route, particles with lifetime $c \tau>10 \mathrm{~mm}$ are decayed by GEANT4 rather than the $\mathrm{MC}$ generator. The majority of the simulation time is spent modelling the complex calorimeter geometry; in some cases the simulation is performed by ATLFAST-II [85], which contains a simplified calorimeter simulation.

Digitisation converts the energy deposition into readout voltages and currents. Following this, the events can be treated like experimental collision events.

\section{Pile-up simulation}

As described in Chapter 3, each LHC bunch crossing can result in soft proton-proton interactions, known as pile-up, in addition to the hard process. This obscures the interesting physics and is important to model accurately.

In-time pile-up (same bunch crossing as the hard process) is modelled by overlaying simulated energy deposits from soft $p p$ interactions generated with PүтнIA 8. The number of overlaid events depends upon the beam conditions (see Section 3.2).

Out-of-time pile-up (different bunch crossing to the hard process) affects detector sub-systems whose latency is longer than the bunch spacing. For such sub-systems, signals from out-of-time pile-up are overlaid with corresponding time shifts; again this depends on the beam conditions.

\subsubsection{Parton shower tuning study}

When studying MC modelling uncertainties in the ggF process (see Section 5.2), a discrepancy was observed at high jet multiplicity between PowhegBox+Pythia 8 and PowhegBox+Pythia 6 (see the green and black lines in Figure 2.4). It is also observed in other electroweak processes. 


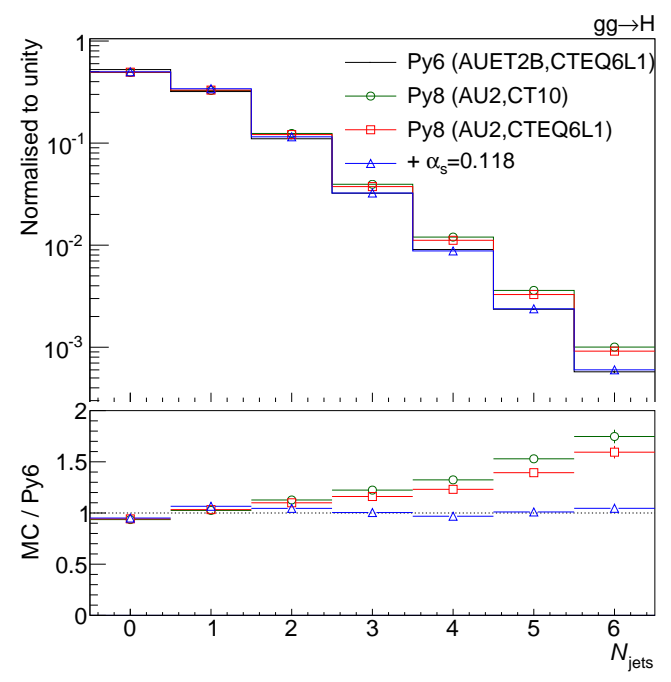

Figure 2.4: Jet multiplicity produced by PowhEgBox+PyThIA 8 with a selection of shower tunes. The green circles correspond to the tune used in the analysis. The red squares change the parton shower PDFs from CT10 to CTEQ6L1. The blue triangles additionally change the parton shower $\alpha_{\mathrm{S}}\left(m_{Z}\right)$ from 0.137 to 0.118 (as used in PowhegBox). PowhegBox+Pythia 6 is shown in black for reference, and is in good agreement with PowhegBox+Herwig (not shown).

The hadronisation and UE models of standalone PYTHIA 8 have been tuned to ATLAS UE data with a variety of PDF sets (known as AU2 tunes) [86]. However, the parton shower was not tuned since the default settings successfully described experimental data.

When modelling ggF with PowhegBox, the AU2-CT10 tune was used in order to match the PDFs used in the matrix element calculation. Technically speaking, a dedicated PowhegBox+Pythia 8 tune should have been used, but this was unavailable. Unfortunately, a couple of issues had a negative impact on the NLO-PS matching. First, the parton shower evolves $\alpha_{\mathrm{S}}$ at LO, whilst NLO PDFs were used in the shower. Second, there was a mismatch between the $\alpha_{\mathrm{S}}$ used in PowhegBox, $\alpha_{\mathrm{S}}\left(m_{Z}\right)=0.118$, and the default value in the parton shower, $\alpha_{\mathrm{S}}\left(m_{Z}\right)=0.137$. The effect of these issues is shown in Figure 2.4.

Identification of this poor matching has led to improvements in the latest round of MC tuning, where dedicated PowhegBox+Pythia 8 tunes are fit using an adjusted parton shower [87]. These shall be used in Run II. 


\subsection{Jet algorithms}

We have seen in Section 2.2 how coloured partons produced in a hard subprocess (in the $\mathrm{ME}$ ) or radiated from the incoming partons (ISR) will each produce a shower of partons, which subsequently hadronise. By measuring the energy and direction of the resulting collimated jet of hadrons, it is possible to infer information about the original quark or gluon. This is very useful for probing the perturbative hard scatter, whilst remaining fairly insensitive to poorly understood hadronisation effects.

A jet algorithm defines how the large number of final state particle four-momenta are grouped into a small number of jet four-momenta. Such an algorithm should satisfy a number of criteria, the most important being infrared and collinear safety [88]. This requires that the jets are insensitive to additional soft or collinear emissions.

Multiple jet algorithms are implemented in the FASTJET software library [89]. In particular, sequential recombination algorithms are popular at the LHC, which iteratively combine the closest pair of particles according to some distance measure $d_{i j}$.

Consider an algorithm where all the inter-particle distances $d_{i j}$ and particle-beam distances $d_{i \mathrm{~B}}$ are calculated. If the minimum of these is a $d_{i j}$ rather than a $d_{i \mathrm{~B}}$, then particles $i$ and $j$ are combined into single new particle. If the minimum is a $d_{i \mathrm{~B}}$, then particle $i$ is declared a jet and removed from the list of particles. Then the algorithm restarts. We define the distances

$$
\begin{aligned}
d_{i j} & =\min \left(p_{\mathrm{T} i}^{2 m}, p_{\mathrm{T} j}^{2 m}\right) \frac{\Delta R_{i j}^{2}}{R^{2}}, \quad \Delta R_{i j}^{2}=\left(y_{i}-y_{j}\right)^{2}+\left(\phi_{i}-\phi_{j}\right)^{2} \\
d_{i \mathrm{~B}} & =p_{\mathrm{T} i}^{2 m}
\end{aligned}
$$

where $p_{\mathrm{T} i}, y_{i}$ and $\phi_{i}$ are the transverse momentum, rapidity and azimuthal angle of particle $i$ with respect to the beam axis, respectively. $R$ and $m$ are parameters of the algorithm, with $R$ effectively determining the size of the jet.

With $m=1$, known as the $k_{\mathrm{T}}$ algorithm, combinations between soft particles are favoured. This follows the evolution of QCD, but leads to rather irregular jet shapes.

With $m=-1$, known as the anti- $k_{\mathrm{T}}$ algorithm [90], combinations between hard particles are favoured. This means the jets grow outwards from a hard 'seed', ultimately producing more circular jets. However, the jet substructure can no longer be used to 
infer details of the jet evolution history. The jets used in this thesis were anti- $k_{\mathrm{T}}$ jets with $R=0.4$, and their reconstruction shall be described in detail in Section 4.2.5. 


\section{Chapter 3}

\section{The ATLAS experiment}

The ATLAS experiment is a general-purpose particle detector operated by an international collaboration of more than 3000 scientists. It is designed to search for a broad range of new phenomena by precisely studying the collisions of high energy protons, which are provided by the Large Hadron Collider (LHC) accelerator at CERN, Geneva.

The LHC and the $p p$ collision data it provides are described in Section 3.1 and Section 3.2 respectively. Then, the ATLAS detector is described in Section 3.3.

\subsection{The Large Hadron Collider}

The LHC is the world's largest and most energetic particle accelerator. It is installed in a $27 \mathrm{~km}$ tunnel at a mean depth of $100 \mathrm{~m}$ beneath the French-Swiss border, which was previously occupied by the Large Electron-Positron collider (LEP). It accelerates beams of protons (or lead ions) to high energy and then collides them within the ATLAS, CMS, LHCb and ALICE detectors. Although the design centre-of-mass (CM) collision energy is $14 \mathrm{TeV}$, it operated at $7 \mathrm{TeV}$ and $8 \mathrm{TeV}$ during Run I, as described in Section 3.2.

The LHC proton beams have humble beginnings as hydrogen molecules in a standard gas bottle, before being stripped of their electrons and entering the LHC accelerator complex shown in Figure 3.1. The LHC accelerator features 16 radio-frequency cavities to provide acceleration and restore energy losses, 1232 dipole magnets to bend the beam into a nearly circular path, 392 quadrupole magnets to focus the beam, and many other 


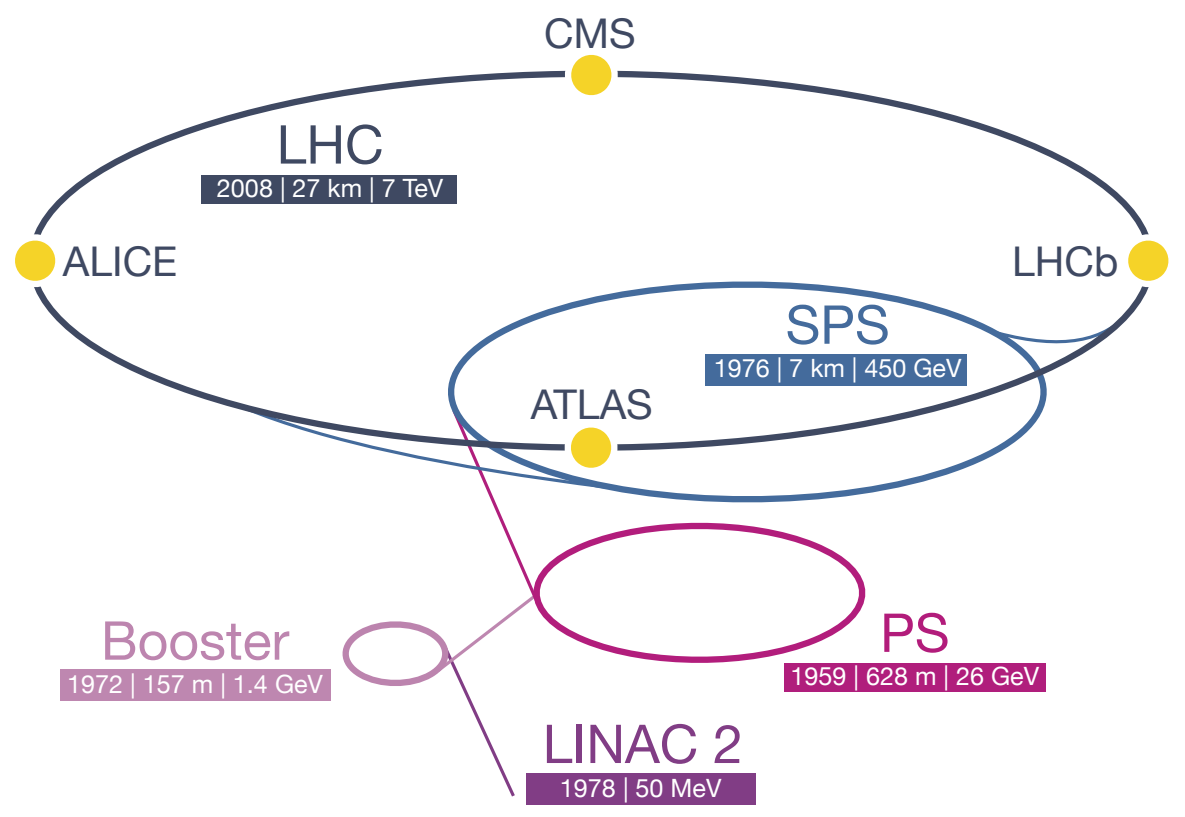

Figure 3.1: The LHC accelerator complex at CERN. The successively higher energy accelerators are: Linear Accelerator 2 (LINAC 2), Proton Synchrotron Booster, Proton Synchrotron (PS), Super Proton Synchrotron (SPS), and finally the Large Hadron Collider (LHC). The four main LHC detectors are also shown.

magnetic components. ${ }^{1}$ Most of these magnets rely on unprecedented superconducting twin-bore magnet technology operating at cryogenic temperatures.

For experiments to be sensitive to rare processes with small cross sections, such as Higgs boson production, the detectors must record a large integrated luminosity. This is seen by considering the expected number of events produced for process $i$

$$
N_{i}=\sigma_{i} \int L \mathrm{~d} t
$$

where $\sigma_{i}$ is the cross section and $L$ is the instantaneous luminosity, a figure of merit for a collider. The instantaneous luminosity can be increased by optimising the parameters of

$$
L=\frac{N_{\mathrm{b}}^{2} n_{\mathrm{b}} f_{\mathrm{rev}}}{4 \pi \Sigma_{x} \Sigma_{y}} F
$$

where $N_{\mathrm{b}}$ is the number of particles per bunch, $n_{\mathrm{b}}$ is the number of bunches per beam, $f_{\text {rev }}$ is the revolution frequency, $\Sigma_{x}$ and $\Sigma_{y}$ are the $x$ and $y$ components of the beam size, and $F$ is a reduction factor due to the crossing angle at the interaction point. The LHC

\footnotetext{
${ }^{1}$ Former DG of CERN Christopher Llewellyn Smith chose the magnet colour to resemble Oxford blue.
} 
is designed to hold 2808 proton bunches, corresponding to $25 \mathrm{~ns}$ bunch spacing, each containing $1.15 \times 10^{11}$ protons. The other design parameters are $\Sigma_{x, y}=16.7 \mu \mathrm{m}$ and $f_{\text {rev }}=11.25 \mathrm{kHz}$, giving a design luminosity of $L \approx 10^{34} \mathrm{~cm}^{-2} \mathrm{~s}^{-1}[91]$.

A trade-off for higher luminosity is a larger number of additional proton-proton interactions, known as pile-up. Although a rare interesting event will trigger the detector readout, these common uninteresting events will simultaneously be recorded, obscuring the interesting physics and degrading detector performance. Increasing $N_{\mathrm{b}}$ gives more interactions within the same bunch crossing, known as in-time pile-up. For large $n_{\mathrm{b}}$, the bunch spacing can be shorter than the detector latency, and interactions from other bunch crossings can affect the measurement; this is known as out-of-time pile-up. Reducing $\Sigma_{x, y}$ will increase both types of pile-up.

\section{2 pp collision data}

It is clear from (3.1) that the luminosity delivered to the detector is a key input when studying $p p$ collisions at the LHC. It is directly proportional to the expected number of events, and uncertainties in its value will be propagated to measured cross sections. The measurement of the luminosity delivered to the ATLAS detector is described in Section 3.2.1, followed by a description of the dataset used in this thesis.

\subsubsection{Luminosity measurement}

Beam losses incurred by the collisions cause the luminosity to decay (a typical run lasts $\approx 10$ hours). Thus, it is necessary to measure the instantaneous luminosity in real-time.

At the LHC, the number of inelastic $p p$ interactions per bunch crossing follows a Poisson distribution, with a mean value $\mu$. As mentioned in Section 3.1, a large luminosity results in $\mu>1$ (a condition known as pile-up). Thus, the luminosity $L$ can be monitored "online" by measuring the observed number of interactions per crossing $\mu_{\mathrm{vis}}$, using [92]

$$
L=\frac{\mu n_{\mathrm{b}} f_{\mathrm{rev}}}{\sigma_{\mathrm{inel}}}=\frac{\mu_{\mathrm{vis}} n_{\mathrm{b}} f_{\mathrm{rev}}}{\sigma_{\mathrm{vis}}}
$$

where $\sigma_{\text {inel }}$ is the inelastic $p p$ cross section. The expression is rewritten with "visible" quantities, owing to inefficiencies in the detector and algorithm used to measure $\mu$. 
The beam conditions monitor (BCM) and LUCID detectors, respectively situated $2 \mathrm{~m}$ and $17 \mathrm{~m}$ down the beamline, each count the number of activated readout channels per bunch crossing, which is highly correlated with $\mu_{\mathrm{vis}}$. The BCM consists of 16 small diamond sensors, and was primarily designed to issue beam-abort requests when beam losses risk damaging the ATLAS detector. LUCID comprises 16 tubes of $\mathrm{C}_{4} \mathrm{~F}_{10}$ gas, which radiate and collect Cherenkov photons when struck by charged particles.

BCM and LUCID are calibrated during dedicated van der Meer (vdM) scans, effectively determining $\sigma_{\text {vis }}$ in (3.3). In a vdM scan, event rates are measured while the beams are separated in steps of known distance, allowing direct measurement of beam sizes $\Sigma_{x}$ and $\Sigma_{y}$. The absolute luminosity is then determined through (3.2). The uncertainty in the $\mathrm{vdM}$ calibration dominates the uncertainty in the delivered luminosity.

Additional methods, such as measuring average particle rates with the ATLAS calorimeters, can be used to improve the luminosity estimation offline.

\subsubsection{Run I dataset}

Data-taking operations during Run I of the LHC were incredibly successful, and some important parameters of the $p p$ datasets are summarised in Table 3.1 and Figure 3.2. These show that a larger dataset was obtained in 2012 compared with 2011, but at the expense of a higher pile-up environment.

\begin{tabular}{lcccc}
\hline & 2010 & 2011 & 2012 & Design \\
\hline Centre-of-mass energy $(\mathrm{TeV})$ & 7 & 7 & 8 & 14 \\
Minimum bunch spacing $(\mathrm{ns})$ & 150 & 50 & 50 & 25 \\
Peak luminosity $\left(10^{33} \mathrm{~cm}^{-2} \mathrm{~s}^{-1}\right)$ & 0.2 & 3.6 & 7.7 & 10 \\
Delivered luminosity $\left(\mathrm{fb}^{-1}\right)$ & 0.047 & 5.46 & 22.8 & - \\
Recorded luminosity $\left(\mathrm{fb}^{-1}\right)$ & 0.044 & 5.08 & 21.3 & - \\
Luminosity uncertainty $\delta L / L$ & $3.5 \%$ & $1.8 \%$ & $2.8 \%$ & - \\
\hline
\end{tabular}

Table 3.1: Summary of $p p$ collision data during LHC Run I. Luminosities use the offline calibration. 


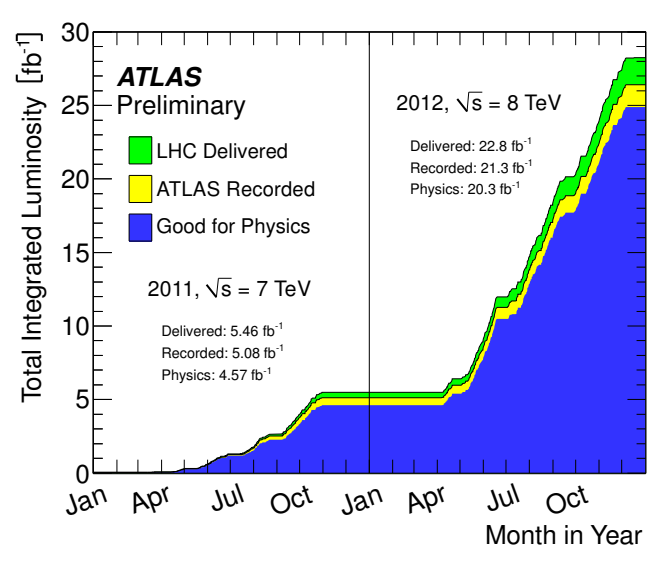

(a) Luminosity

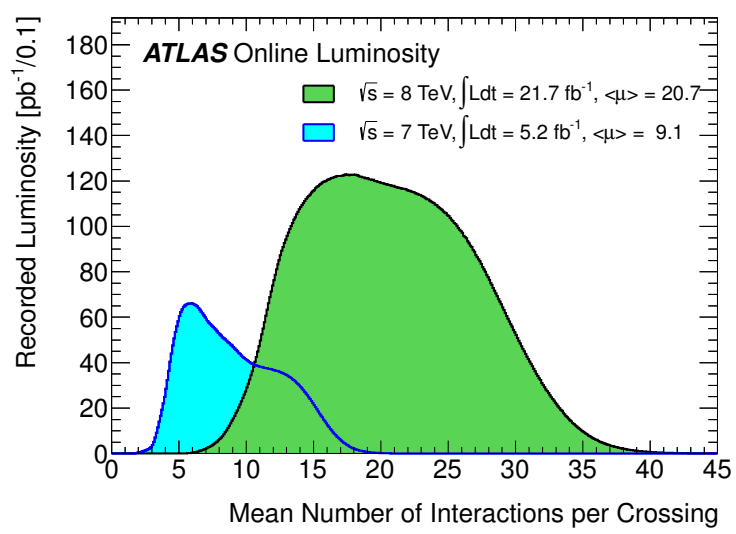

(b) Pile-up

Figure 3.2: (a) Cumulative luminosity delivered (green), recorded (yellow), and declared 'good for physics' (blue) during 2011 and 2012. Luminosities use the offline calibration. (b) Mean number of interactions per bunch crossing $\mu$ for the 2011 (blue) and 2012 (green) datasets, calculated with an inelastic $p p$ cross section of $71.5 \mathrm{mb}$ at $\sqrt{s}=7 \mathrm{TeV}$ and $73.0 \mathrm{mb}$ at $\sqrt{s}=8 \mathrm{TeV}$. Luminosities use the online calibration.

\subsection{The ATLAS detector}

ATLAS is a general-purpose particle detector for probing hadron-hadron collisions [93], from precise measurements of Standard Model (SM) processes to searches for signatures of new physics. As such, it must achieve a good performance in the reconstruction of all physics objects interacting with the detector (leptons, photons and jets), and infer the existence of non-interacting particles through transverse momentum imbalance. A good vertex resolution is needed for jet flavour tagging and to distinguish pile-up events. Other requirements include a fast trigger system to select interesting events to record.

The design of ATLAS exhibits cylindrical and forward-backward symmetries with the nominal interaction point at the centre. ${ }^{2}$ The three detector sub-systems (tracking, calorimetry and the muon spectrometer) follow a consistent design of a central barrel with end-caps at both ends, giving a high degree of hermeticity (see Figure 3.3). Barrel components are arranged on concentric cylinders around the beam axis, while end-cap components are on disks perpendicular to the beam axis.

\footnotetext{
${ }^{2}$ ATLAS uses a right-handed coordinate system with its origin at the nominal interaction point. The $x$-axis points to the centre of the LHC ring, the $y$-axis points upwards, and the $z$-axis points along the beam line. Positions and directions within the detector are given in spherical coordinates $(r, \theta, \phi)$ where $r$ is the radial distance, $\theta$ is the polar angle and $\phi$ is the azimuthal angle. Usually the polar angle is replaced with pseudorapidity $\eta=-\ln \tan (\theta / 2)$. The distance between two positions in $\eta$ - $\phi$ space is $\Delta R=\sqrt{\Delta \eta^{2}+\Delta \phi^{2}}$. Observables labelled "transverse" are projected into the $x-y$ plane.
} 


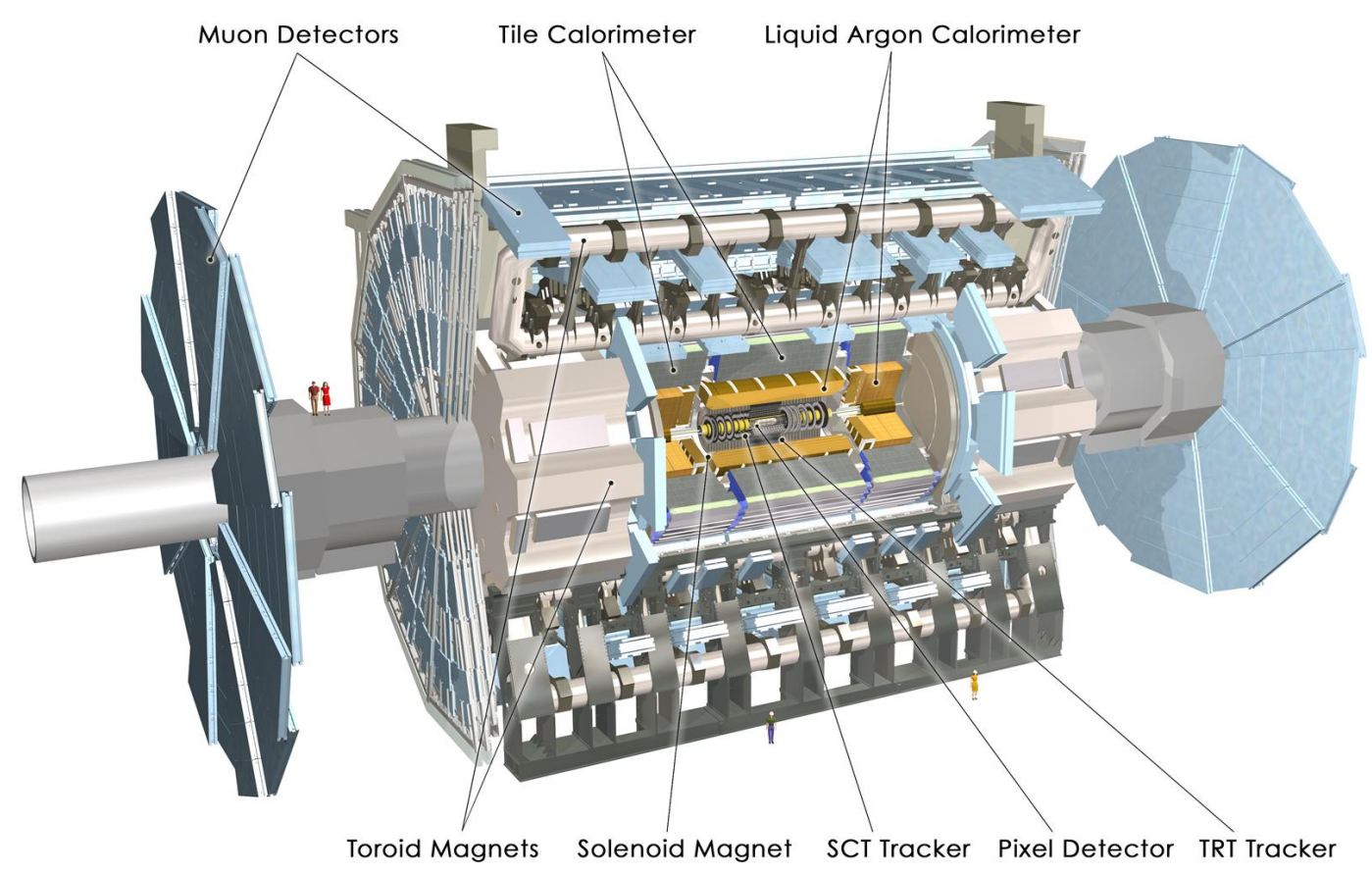

Figure 3.3: Cut-away view of the ATLAS detector [93]. It has a height of $25 \mathrm{~m}$, a length of $44 \mathrm{~m}$ and weighs 7000 tonnes.

Figure 3.4 shows how different particles interact with the various parts of the detector. Next to the beam pipe is the inner detector which precisely tracks the trajectories of charged particles. Then there are the electromagnetic and hadronic calorimeters which absorb and measure the energy of interacting particles. Since high- $p_{\mathrm{T}}$ muons act as minimum ionising particles, they survive the calorimeters and are tracked by the muon spectrometer. Superconducting solenoid and toroid magnets provide magnetic fields to the inner detector and muon spectrometer respectively, allowing the momenta of charged particles to be measured from the curvature of their tracks.

\subsubsection{Tracking}

Pattern recognition algorithms are used to precisely track the trajectories of charged particles through the inner detector (ID), shown in Figure 3.5. The ID is immersed in a $2 \mathrm{~T}$ solenoidal magnetic field, which enables the momentum of a particle to be measured from the curvature of its track. Momentum and vertex measurements both require an excellent spatial resolution, which is achieved through fine detector granularity. The ID covers the region $|\eta|<2.5$ and consists of three complementary sub-detectors: 


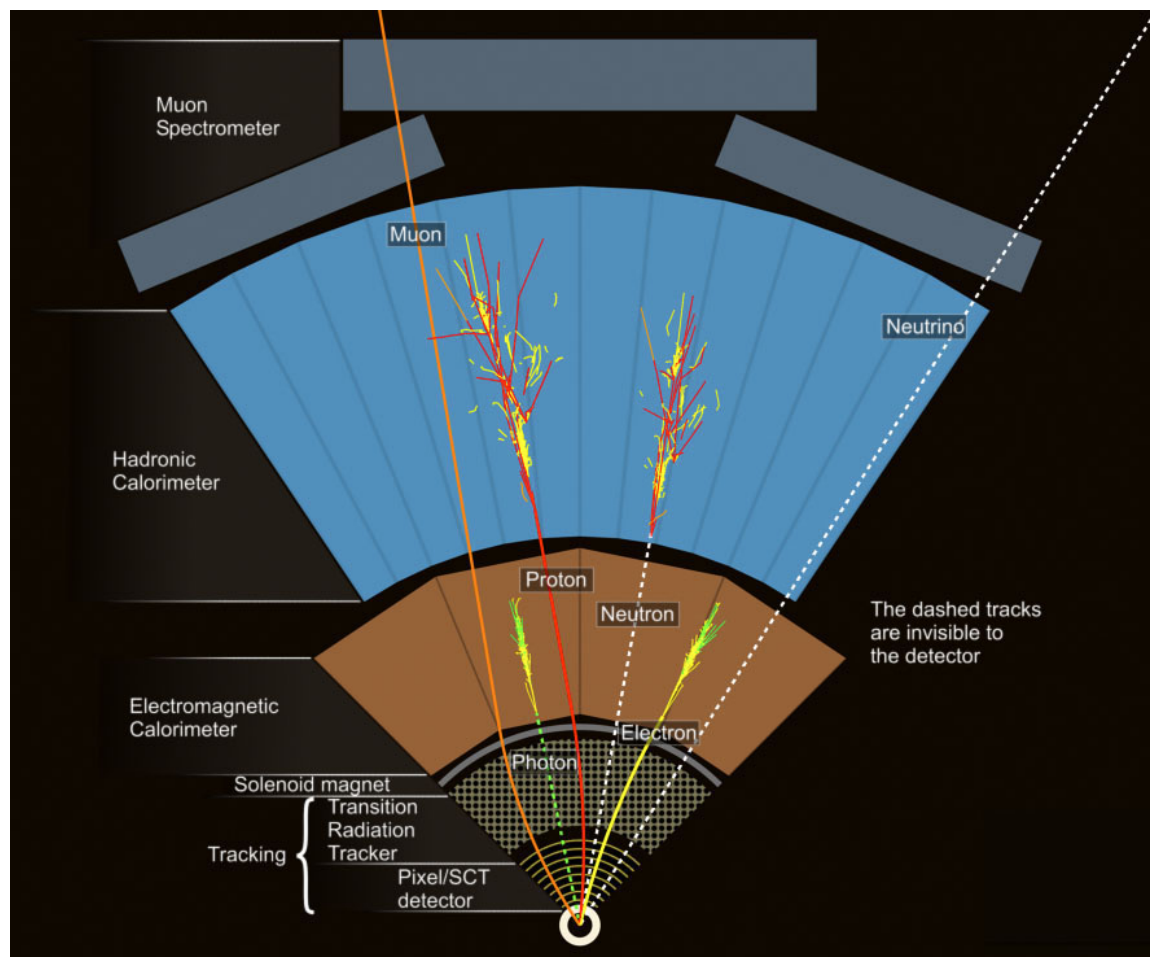

Figure 3.4: Cross-sectional view of ATLAS, showing how different particles interact with the sub-detectors. ATLAS Experiment (C) 2013 CERN.
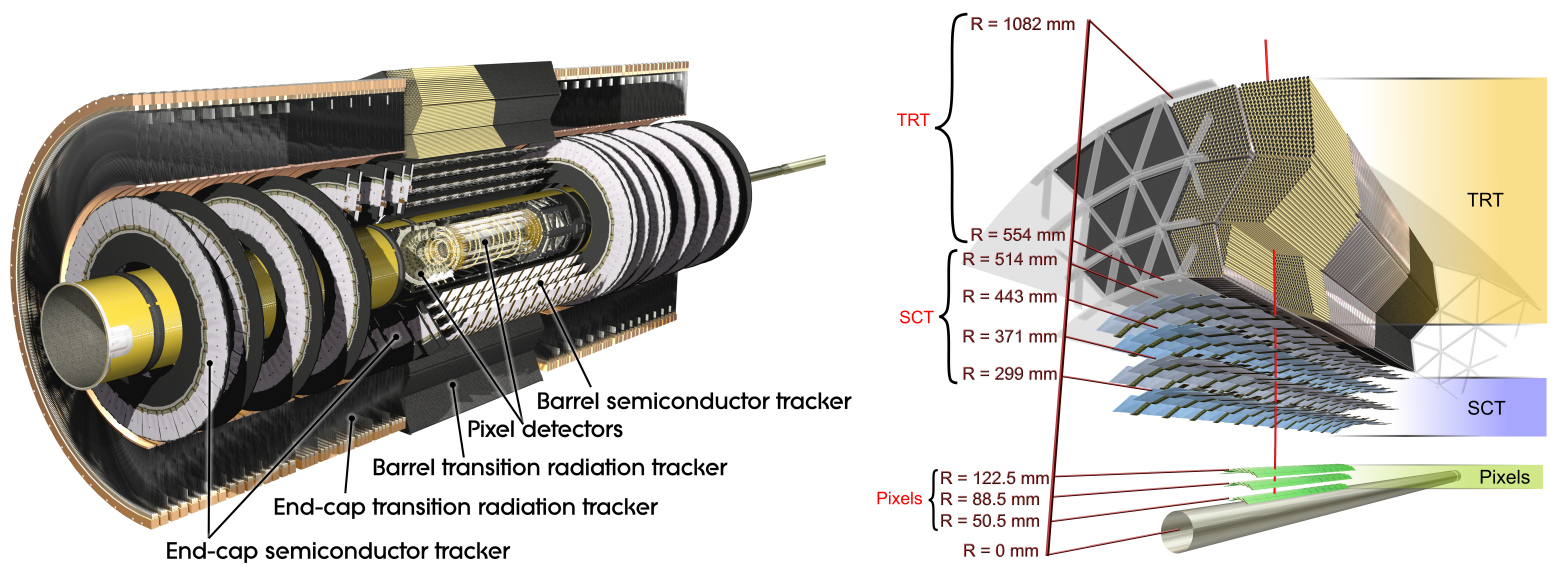

Figure 3.5: The ATLAS inner detector: cut-away view (left) and cross-sectional view showing a trajectory through the three sub-detectors (right) [93]. The inner detector has a length of $6.2 \mathrm{~m}$ and a radius of $1.1 \mathrm{~m}$. The beam pipe radius is $29 \mathrm{~mm}$. 


\section{Pixel detector}

The pixel detector is installed in three layers closest to the beam pipe, and therefore requires the highest granularity to handle the large particle fluxes. It comprises more than 80 million silicon pixels, each of area $50 \times 400 \mu^{2}$ and thickness $250 \mu \mathrm{m}$. The intrinsic accuracy is $10 \mu \mathrm{m} \times 115 \mu \mathrm{m}$ in $r \phi-z(r \phi-r)$ space in the barrel (end-cap).

A silicon particle detector consists of a reverse-biased p-n junction. When a charged particle passes through the depletion region it creates an electron-hole pair, which travel to the respective electrodes and produce a signal current.

\section{Semiconductor tracker (SCT)}

The SCT features 15,912 silicon strip sensors, each consisting of 770 strips with a pitch of $80 \mu \mathrm{m}$ and a length of $6 \mathrm{~cm}$. Pairs of sensors are sandwiched together into modules with a stereo angle of $40 \mathrm{mrad}$, enabling the coordinate parallel to the strip to be measured. There are four (nine) layers of modules in the barrel (end-cap), which ensures that each track passes through at least four modules. The intrinsic accuracy is $17 \mu \mathrm{m} \times 580 \mu \mathrm{m}$ in $r \phi-z(r \phi-r)$ space for the barrel (end-cap).

\section{Transition radiation tracker (TRT)}

The TRT features 370,000 drift chambers, known as straws, which simultaneously function as a straw tracker to enhance particle tracking and as a transition radiation detector to aid electron identification. Straws are aligned with the beam pipe in the barrel and radially in the end-caps. The TRT covers the region $|\eta|<2.0$.

Each $4 \mathrm{~mm}$ diameter straw has a Kapton ${ }^{\circledR}$ wall with a conductive coating, which acts as a cathode at $-1530 \mathrm{~V}$, and a central tungsten wire anode. The straws are filled with a xenon-based gas mixture that is ionised by a traversing charged particle. The freed electrons drift to the anode and produce a signal current. The drift time is used to measure the impact parameter of the incident charged particle relative to the anode and, since a track typically traverses 36 straws, collectively this information yields an intrinsic accuracy of $130 \mu \mathrm{m}$ in $r \phi$ (much smaller than the straw diameter). However, there is no tracking information parallel to the straw.

The layers of straws are interleaved with polypropylene radiator fibres or foils, and the changes in refractive index cause charged particles to emit X-ray transition radiation (TR). The TR is absorbed by the xenon gas in the straws; TR signals are distinguished from tracking signals by a higher threshold. Since the probability of TR is proportional to the particle's $\gamma$-factor, for a given energy lighter particles produce more TR than heavier particles. This aids electron-pion discrimination. 


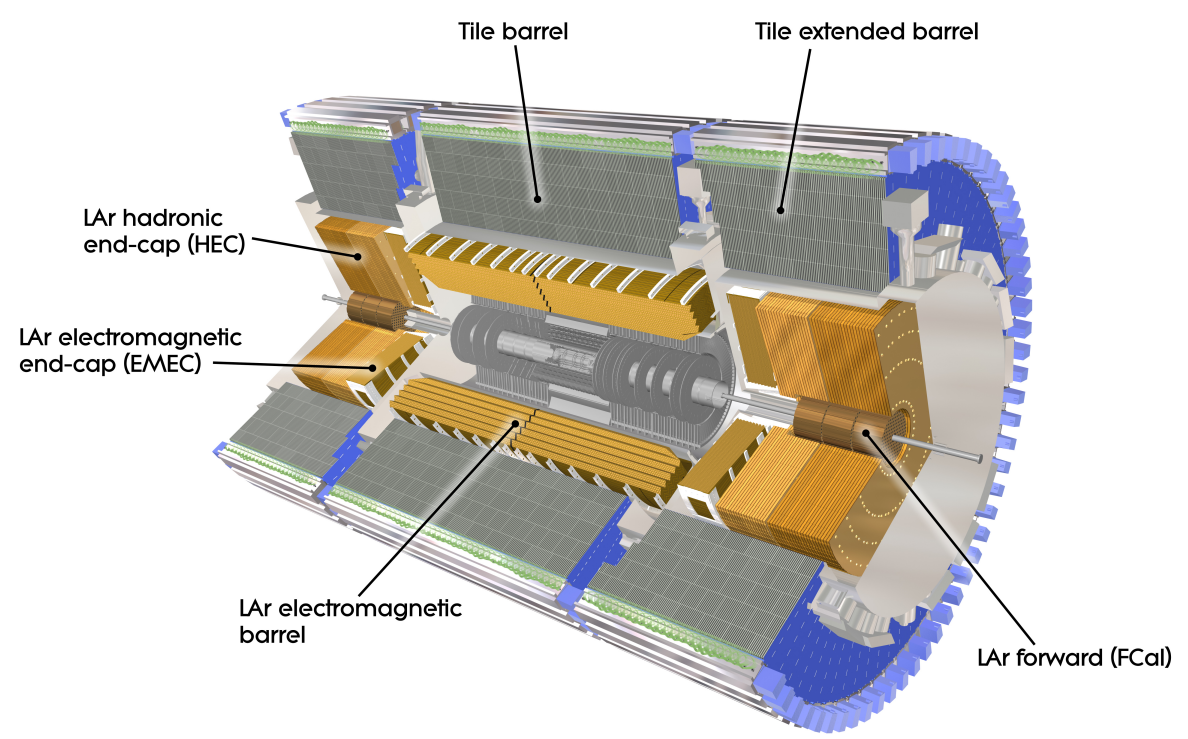

Figure 3.6: Cut-away view of the ATLAS calorimeter system [93].

\subsubsection{Calorimetry}

Particle energies are measured with sampling calorimeters, which consist of alternating layers of absorber and active material. The dense absorber causes energy deposition via a particle shower, whilst the active material produces a signal proportional to the sampled energy. The length scale of energy loss is the radiation length $X_{0}$ for electrons and photons ${ }^{3}$ and the nuclear interaction length $\lambda$ for hadronic showers ${ }^{4}$, while muons act as minimum ionising particles and escape the calorimeters. Since punch-through into the muon system must be minimised, $X_{0}$ and $\lambda$ set the size of the calorimeter. Conversely, limits to detector size constrain the materials that can be used.

The ATLAS calorimeter consists of an inner electromagnetic calorimeter (for electrons and photons) and an outer hadronic calorimeter (for jets), as shown in Figure 3.6. They cover the regions $|\eta|<3.2$ and $|\eta|<4.9$ respectively. The total thickness of the electromagnetic calorimeter corresponds to more than $22 X_{0}$ and the total thickness of the entire calorimeter corresponds to more than $10 \lambda$.

${ }^{3}$ High-energy electrons (photons) mostly lose energy in matter by bremsstrahlung $\left(e^{+} e^{-}\right.$pair production). In this regime, $X_{0}$ is (a) the mean distance in which an electron loses all but $e^{-1}$ of its energy, (b) $\frac{7}{9}$ of the mean free path of a photon, and (c) the characteristic scale of electromagnetic showers.

4 Hadrons lose energy in matter through inelastic hadronic interactions, forming hadronic showers (though neutral pions create electromagnetic showers). $\lambda$ is (a) the mean free path of a hadron, and (b) the characteristic scale of hadronic showers. 


\section{Electromagnetic calorimeter (ECal)}

The ECal uses a lead absorber and a liquid argon (LAr) active material arranged in an accordion geometry, which ensures uniform $\phi$-coverage. The LAr is ionised by charged particles, and the freed electrons produce a signal current in the readout electrodes. LAr was chosen for its linear behaviour, stability and radiation-hardness.

A fine granularity is required to make precise electron and photon measurements, and to distinguish single photons from $\pi^{0} \rightarrow \gamma \gamma$. Thus, the ECal is typically segmented into three layers with a minimum $\Delta \eta \times \Delta \phi$ granularity of $0.003 \times 0.025$. The transition between the barrel and the end-cap, $1.37<|\eta|<1.52$, is used for detector services. Since this material is difficult to model, this "crack" region is usually excluded when reconstructing electrons.

For $|\eta|<1.8$, a LAr presampler is installed before the first lead layer. This enables estimation of the energy lost by electrons and photons before encountering the ECal.

\section{Hadronic calorimeter (HCal)}

The HCal consists of a tile calorimeter covering $|\eta|<1.7$, a hadronic end-cap (HEC) covering $1.5<|\eta|<3.2$, and a forward calorimeter (FCal) covering $3.1<|\eta|<4.9$. The HCal has a coarser granularity than the ECal: it is typically segmented into three layers with a minimum $\Delta \eta \times \Delta \phi$ granularity of $0.1 \times 0.1$.

The tile calorimeter uses a steel absorber with scintillator tiles as the active material. The scintillation light is collected by optical fibres and turned into a signal by photomultiplier tubes. The HEC and FCal both use LAr active material. The HEC uses a copper absorber, whereas the FCal uses copper in the first layer (for electromagnetic measurements) and tungsten in the two subsequent layers. 


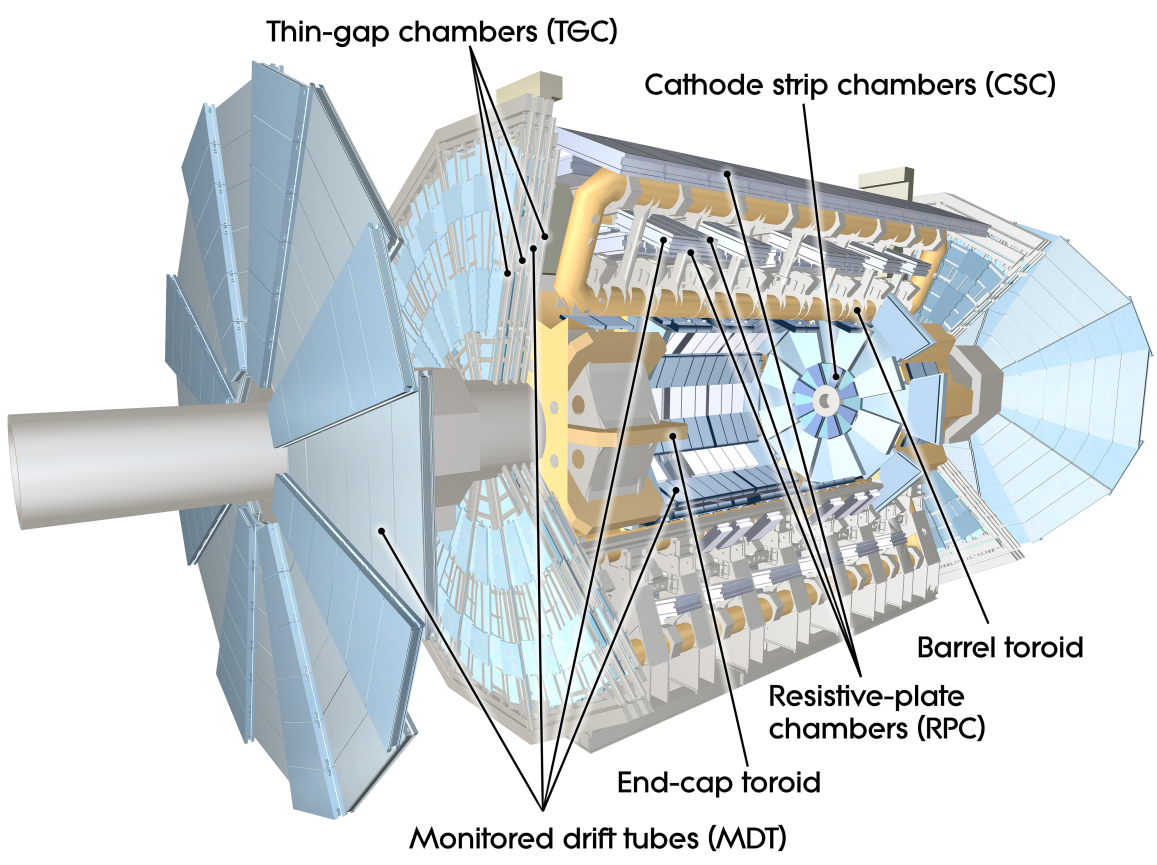

Figure 3.7: Cut-away view of the ATLAS muon spectrometer [93].

\subsubsection{Muon spectrometer}

The muon spectrometer (MS) provides precise tracking of muons that have exited the calorimeters. A huge air-core toroid magnet system generates a $0.5 \mathrm{~T}(1 \mathrm{~T})$ field in the barrel (end-caps), enabling momentum to be inferred from track curvature measurements. Four types of tracking chamber are installed in three layers (see Figure 3.7):

\section{Monitored drift tubes (MDTs) and cathode strip chambers (CSCs)}

MDTs and CSCs provide precise momentum measurement, with a resolution of about $40 \mu \mathrm{m}$ in the bending plane. MDTs cover the region $|\eta|<2.7$, but the innermost end-cap is replaced with CSCs to withstand the higher particle flux. CSCs are multiwire proportional chambers with cathode planes segmented into strips in orthogonal directions.

\section{Resistive plate chambers (RPCs) and thin gap chambers (TGCs)}

RPCs and TGCs provide less precise tracking, but at a faster readout speed needed for triggering. They also provide orthogonal coordinates to those of the MDTs and CSCs. RPCs are gas-filled parallel plate detectors operated in avalanche mode. TGCs are multiwire proportional chambers operated in quasi-saturated mode. 


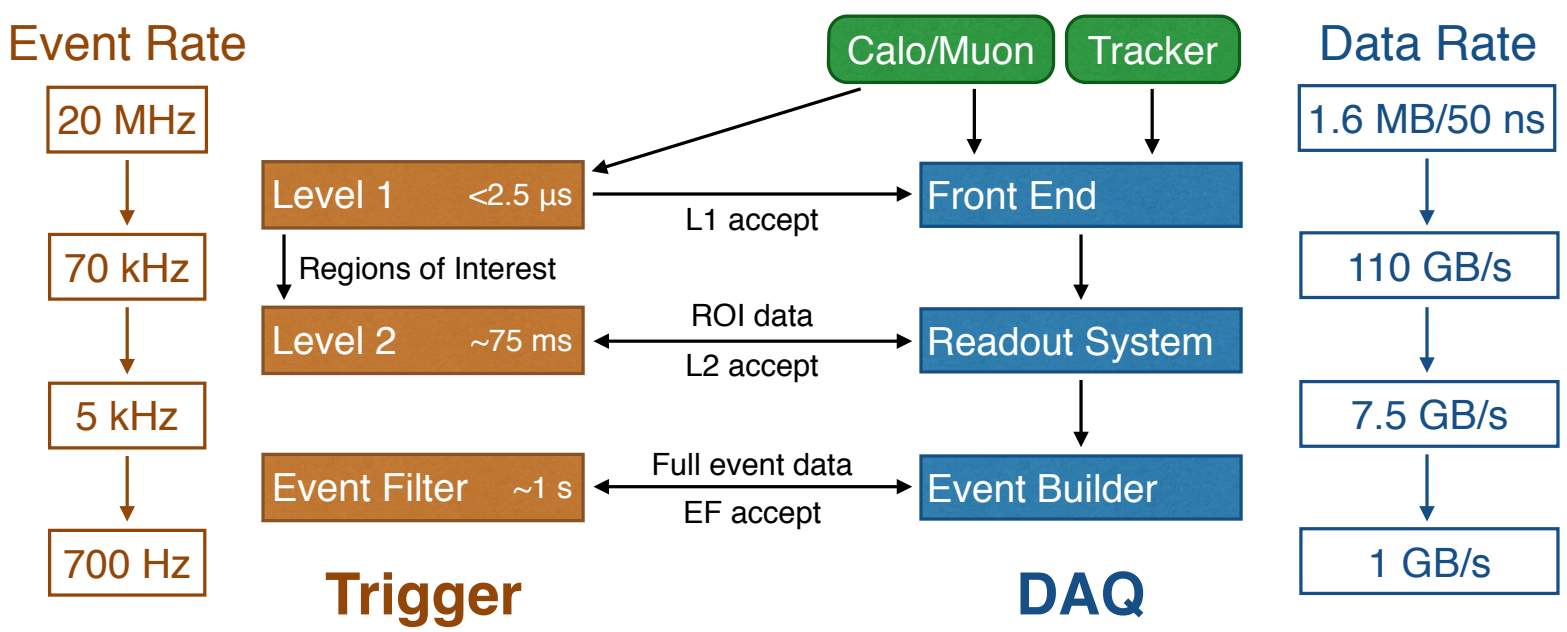

Figure 3.8: Schematic diagram of the ATLAS trigger and data acquisition (DAQ) system. Typical values for event rates, data rates and trigger latencies during the 2012 run are taken from [94].

\subsubsection{Trigger and data acquisition}

It is technically infeasible to record the detector readout (1.6 MB per event) at the bunch crossing rate expected at the $\mathrm{LHC}(40 \mathrm{MHz})$. Moreover, the majority of these events are uninteresting in terms of the LHC physics program. For these reasons, ATLAS has a trigger system to identify and retain interesting events for further analysis offline.

The trigger is split into three levels with successively lower event rates: level 1 (L1), level 2 (L2) and the event filter (EF). This is achieved by affording a longer latency for later levels to apply more refined selection criteria, as shown in Figure 3.8. The L2 and EF triggers are collectively referred to as the high level trigger (HLT), and are implemented on an off-detector computer farm.

The L1 trigger searches for high- $p_{\mathrm{T}}$ leptons, photons and jets, in addition to events with significant total transverse energy or transverse energy imbalance. The decision is made using muon trigger chamber information (RPCs and TGCs) and reduced-granularity calorimeter information. Since the decision latency $(2.5 \mu \mathrm{s})$ is longer than the bunch spacing (25 ns), event data is stored in pipelines while a decision is made.

The detector coordinates of interesting features identified by the L1 trigger, known as regions of interest (ROIs), are passed on to the L2 trigger. The L2 trigger then requests the full degree of detector information for these ROIs ( $\sim 2 \%$ of total event data), in order to make a more informed decision. Finally, the EF trigger has access to the full event 
data, and further reduces the data rate such that it can be written to tape. The entire trigger system reduces the data rate from $64 \mathrm{~TB} / \mathrm{s}$ to about $1 \mathrm{~GB} / \mathrm{s}$.

\subsubsection{Detector performance}

The performance of each sub-detector is shown in Table 3.2. The $\sigma_{p_{\mathrm{T}}} / p_{\mathrm{T}}$ of the tracker has a term proportional to $p_{\mathrm{T}}$ due to the intrinsic spatial resolution of the detector, and a constant term due to multiple scattering. The $\sigma_{E} / E$ of the calorimeters has a constant term due to non-uniformities in the response, and a term proportional to $1 / \sqrt{E}$ due to statistical fluctuations in the hadronic shower (the stochastic term).

\begin{tabular}{lccc}
\hline \multirow{2}{*}{ Detector component } & Resolution & \multicolumn{2}{c}{$|\eta|$ coverage } \\
& & Measurement & L1 trigger \\
\hline Tracker & $\sigma_{p_{\mathrm{T}}} / p_{\mathrm{T}}=0.03 \% \times p_{\mathrm{T}} \oplus 1.5 \%$ & $<2.5$ & - \\
EM calorimeter & $\sigma_{E} / E=10 \% / \sqrt{E} \oplus 1 \%$ & $<3.2$ & $<2.5$ \\
Hadronic calorimeter & $\sigma_{E} / E=50 \% / \sqrt{E} \oplus 3 \%$ & $<4.9$ & $<4.9$ \\
Muon spectrometer & $\sigma_{p_{\mathrm{T}}} / p_{\mathrm{T}}=4 \%$ at $p_{\mathrm{T}}=100 \mathrm{GeV}$ & $<2.7$ & $<2.4$ \\
\hline
\end{tabular}

Table 3.2: The measured performance in the central region of each of the ATLAS subdetectors [95-98]. Energies and momenta are given in GeV. The quoted MS performance is independent of the ID. The contribution of noise to $\sigma_{E} / E$ (a term proportional to $1 / E$ ) is neglected as it depends upon the pile-up environment. 


\section{Chapter 4}

\section{Overview of the $\mathrm{H} \rightarrow \mathrm{WW}$ analysis}

The $W W$ decay of the Higgs boson is a promising search channel as it has a large branching ratio (BR) for a wide range of $m_{H}$. In fact, it is the most probable decay for $m_{H}>135 \mathrm{GeV}$ (see Figure 1.5). This chapter will describe the experimental search for $g g \rightarrow H \rightarrow W W \rightarrow \ell \nu \ell \nu$ (where $\ell=e, \mu$ ) using the 2012 dataset of $p p$ collisions at $\sqrt{s}=8 \mathrm{TeV}$. The search strategy is optimised for a low mass Higgs boson, as favoured by electroweak fits, and thus accounts for off-shell $W$ bosons.

The experimental signature of this search involves electrons, muons, jets and transverse momentum imbalance, and is outlined in Section 4.1. Then, Section 4.2 details how each of these objects is reconstructed by the ATLAS detector. Finally, Section 4.3 describes the criteria by which Higgs boson events are selected and background events are rejected. In doing so, it is necessary to make reference to many exclusive observables of signal and background processes. The modelling and estimation of these observables shall be described in Chapters 5, 6 and 7.

\subsection{Experimental signature}

Following the $H \rightarrow W W$ decay, each $W$ boson will decay leptonically or hadronically, with $\mathrm{BR}(W \rightarrow \ell \nu)=10.8 \%$ and $\mathrm{BR}(W \rightarrow$ hadrons $)=67.6 \%$ [26]. Thus, dileptonic, semi-leptonic and hadronic final states are conceivable. Although the dileptonic channel is suppressed by BRs, it is ultimately the most sensitive as the other two have larger 


\begin{tabular}{ll}
\hline Background & Mechanism of $\ell \ell+p_{\mathrm{T}}^{\text {inv }}$ signature \\
\hline$W W$ & irreducible \\
$t \bar{t}, t W$ & irreducible \\
$t b, t b q$ & jet fakes lepton \\
$Z / \gamma^{*} \rightarrow \ell \ell$ & fake $p_{\mathrm{T}}^{\text {inv }}$ \\
$Z / \gamma^{*} \rightarrow \tau \tau$ & irreducible (for leptonic $\tau$ decays) \\
$W+$ jet, dijet & jet(s) fake lepton(s) \\
$W \gamma$ & photon fakes electron \\
$W Z, W \gamma^{*}, Z Z$ & unreconstructed lepton(s) \\
\hline
\end{tabular}

Table 4.1: Summary of how each background produces the $\ell \ell+p_{\mathrm{T}}^{\text {inv }}$ experimental signature, inclusive in the number of jets.

backgrounds. This chapter will describe the dileptonic search, and henceforth 'lepton' and $\ell$ shall refer to an electron or muon. ${ }^{1}$

Electroweak fits favour a Higgs boson with mass $m_{H}<2 m_{W}$ (see Figure 1.6). It is therefore important for the $H \rightarrow W W$ search to be sensitive to off-shell $W$ bosons. Experimentally, this means using leptons with low $p_{\mathrm{T}}$ thresholds, which unfortunately have reduced purity due to large misidentified hadronic backgrounds.

Since neutrinos do not interact with the detector, it is only possible to infer their combined transverse momentum from an imbalance in the visible momenta, called $p_{\mathrm{T}}^{\mathrm{inv}}$. Thus, it is not possible to fully reconstruct a mass peak in the $H \rightarrow W W \rightarrow \ell \nu \ell \nu$ search, so to be sensitive to the Higgs boson it becomes crucial to accurately understand the many background processes.

The basic experimental signature is two oppositely charged leptons and significant $p_{T}^{\text {inv }}$. However, there are background processes that exhibit the same signature. Others have an aspect of the signature faked by mismeasurement, or some part of their final state is not reconstructed. Table 4.1 introduces the different backgrounds. Jets are a convenient way to separate the contributions of different background processes.

\footnotetext{
${ }^{1}$ Events with one or two $W \rightarrow \tau \nu$ decays can contribute to the dileptonic search when the $\tau$ decays to an electron or muon. This contribution is small however, since $\operatorname{BR}\left(\tau \rightarrow \ell \nu_{\ell} \nu_{\tau}\right)=17.6 \%$ [26]. Also, the kinematics of such events are different due to the additional decay(s) and neutrinos.
} 
Jets can also be used to separate the gluon-gluon fusion $(\mathrm{ggF})$ and vector boson fusion (VBF) production modes of the Higgs boson (see Section 1.3). The search described below is designed for the ggF production mode, and this shall become particularly apparent when describing the $\geq 2$-jet bin in Section 4.3.7.

\subsection{Reconstruction of physics objects}

Relating raw detector output to physical particles is an incredibly difficult task, particularly in a high pile-up environment. Sophisticated algorithms were developed for this task, and are outlined below.

\subsubsection{Tracks}

A track is a sequence of hits in the inner detector (ID) indicative of a charged particle trajectory. To first approximation the trajectories are helical, owing to the pervading solenoidal magnetic field. However, multiple scattering and energy losses can cause significant deviations from this path. Track reconstruction is possible in the region $|\eta|<2.5$ (the extent of the ID).

The inputs to the track reconstruction are threefold: coordinates from the pixel and SCT, ${ }^{2}$ and TRT drift circles. The inside-out algorithm [99, 100] uses a Kalman filter to seed tracks from hits in the three pixel layers and the first SCT layer. The seeds are then extended into the SCT and the TRT and fitted, whilst resolving ambiguities and applying quality criteria. Finally, the outside-in algorithm [99] considers unused track segments in the TRT, and extrapolates them into the SCT and pixel detector. This improves the tracking of secondary particles with a displaced vertex.

\subsubsection{Primary and secondary vertices}

A vertex is a location from which at least two outgoing tracks are reconstructed. Primary vertices are associated with the interactions of incoming protons, whereas secondary vertices are caused by particle decay or photon conversion $\left(e^{+} e^{-}\right.$pair production).

\footnotetext{
2 Each SCT module comprises two layers of silicon strips with a small stereo angle, enabling the
} three-dimensional coordinate to be precisely measured (see Section 3.3.1). 
Primary vertex reconstruction has two steps: association of tracks to vertices (vertex finding), and reconstruction of the vertex position itself (vertex fitting). ATLAS employs an iterative finding-through-fitting algorithm to simultaneously perform both steps [101,102]. First, tracks originating from the interaction region $(\Delta z \approx \pm 5.6 \mathrm{~cm}$ and $\Delta r \approx 15 \mu \mathrm{m})$ are identified and used to seed and fit a single vertex. Tracks considered outliers are then used to seed a new vertex, and a second fit of the two vertices is performed. The algorithm iterates, increasing the number of vertices, until the result stabilises.

The total number of primary vertices $N_{\mathrm{PV}}$ is used to assess the pile-up conditions of the bunch crossing. The primary vertex of the hard scatter is chosen to be that with the highest $\sum p_{\mathrm{T}}^{2}$ of the constituent tracks, and is referred to as simply the primary vertex. As an additional quality criterion, this vertex must have at least three associated tracks.

Secondary vertex reconstruction is highly constrained by the physics of the vertex [102]. This can be enforced by mass or angular constraints, or in the track selection. For example, photon conversions are found using oppositely charged track pairs associated with electrons (via TRT identification), with small opening angle. Flavour tagging of jets, which uses secondary vertices, shall be described in Section 4.2.6.

\subsubsection{Electrons}

Electrons will pass through the ID before being absorbed by the electromagnetic calorimeter (ECal). They are therefore reconstructed by matching a track with an energy cluster in the ECal. Following reconstruction, the vast majority of electron objects are actually misidentified hadrons, whilst many others are from photon conversions or electrons from heavy flavour decay (see Figure 4.1). However, in the $H \rightarrow W W \rightarrow \ell \nu \ell \nu$ search, it is prompt electrons from the hard scatter that are of interest. Therefore identification and isolation criteria are applied to reject these backgrounds and select prompt electrons. The criteria were chosen to optimise the analysis sensitivity, e.g. a low $E_{\mathrm{T}}$ threshold of $10 \mathrm{GeV}$ gives a large signal acceptance but must be compensated by tight identification requirements to reject the large $W+$ jet and dijet backgrounds.

Electrons with $E_{\mathrm{T}}>10 \mathrm{GeV}$ and $|\eta|<2.47$ are selected. Additionally, electrons with $1.37<|\eta|<1.52$ are vetoed since they correspond to the crack region (see Section 3.3.2). 


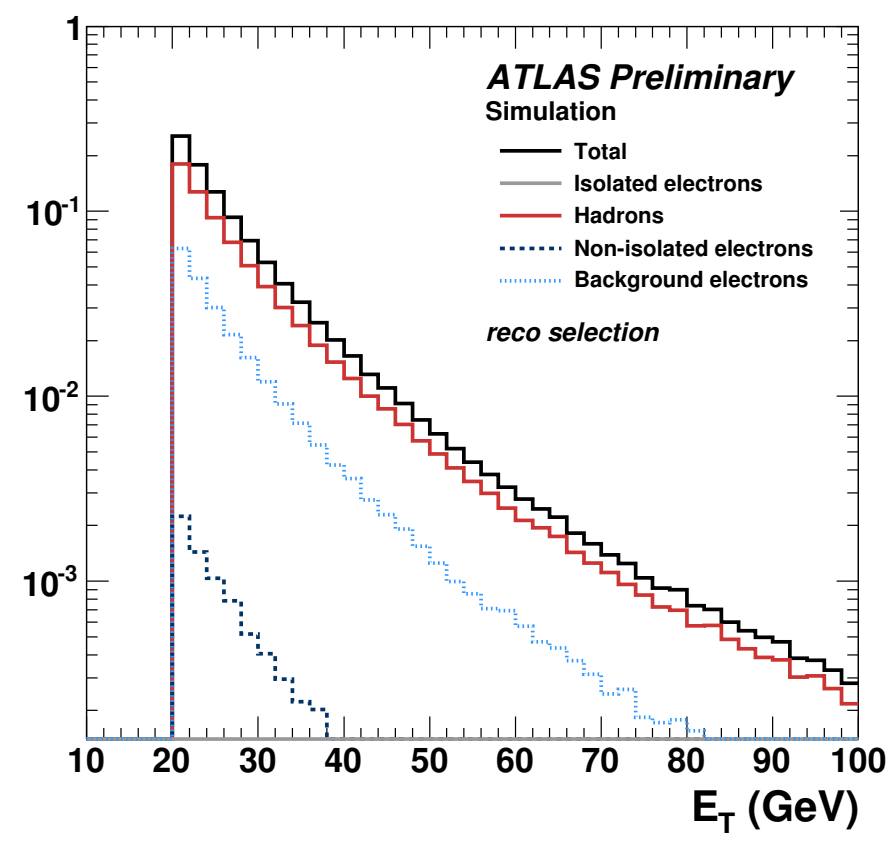

Figure 4.1: The composition of reconstructed electron candidates as a function of transverse energy, simulated by a Pүтніа 6 sample of dijet, heavy flavour, prompt photon, $W$ boson and $Z$ boson production [103]. Non-isolated electrons are from heavy flavour decay. Background electrons are from photon conversions. Isolated electrons are prompt electrons from a $W$ or $Z$ boson, though appear below the y-axis range. The total integral is normalised to unity. 


\section{Reconstruction}

Energy deposits in ECal cells are clustered by a sliding window algorithm [103]. First, the calorimeter is divided into towers of size $\Delta \eta \times \Delta \phi=0.025 \times 0.025$ (the cell size of the middle layer). Then a window of $3 \times 5$ towers in $\eta$ - $\phi$ space scans the ECal for local maxima with $E_{\mathrm{T}}>2.5 \mathrm{GeV}$.

Tracks within $\Delta R<0.3$ of a cluster are then refit using a Gaussian sum filter (GSF) [104]. The default fit described in Section 4.2.1 uses a pion hypothesis to estimate material effects, and does not account for the significant bremsstrahlung experienced by electrons (which is highly $\eta$-dependent). The GSF is a non-linear fitter and improves the accuracy of electron tracking by accounting for this.

For a cluster to be reconstructed as an electron, it must be matched to a track. The matching uses an asymmetric $\Delta \phi$ requirement to allow for increased bending due to bremsstrahlung. If a track is within $\Delta \phi<0.1(0.05)$ and $\Delta \eta<0.05$ of the cluster centre, it is considered matched. When multiple tracks are matched, that with the smallest $\Delta R$ is chosen.

Finally, the cluster is rebuilt using a $3 \times 7(5 \times 5)$ sliding window in the barrel (endcap), and the electron four-momentum is defined [105]. The direction is taken from the matched track and the energy is the cluster energy, corrected for losses in passive material and leakage outside the cluster. These simulation-based corrections depend on the longitudinal shower shape and energy deposited in the presampler. The EM energy scale was calibrated using test beam and in situ $Z \rightarrow e e$ measurements.

\section{Identification}

Electron identification is improved by applying cuts on track quality and shower shape variables. There are three reference operating points called loose, medium and tight, with progressively greater background rejection and lower signal efficiency.

For $E_{\mathrm{T}}>25 \mathrm{GeV}$ the medium criteria are used, comprising cuts on

- shower shape variables (lateral and longitudinal),

- leakage to the hadronic calorimeter (HCal),

- the number of pixel and SCT hits,

- the transverse impact parameter with respect to the primary vertex, $d_{0}$,

- track-cluster matching,

- transition radiation. 


\begin{tabular}{cll}
\hline Electron $E_{\mathrm{T}}(\mathrm{GeV})$ & Tracker isolation & Calorimeter isolation \\
\hline $10-15$ & $p_{\mathrm{T}}^{\text {cone }}(0.4) / E_{\mathrm{T}}<0.06$ & $E_{\mathrm{T}}^{\text {cone }}(0.3) / E_{\mathrm{T}}<0.20$ \\
$15-20$ & $p_{\mathrm{T}}^{\text {cone }}(0.3) / E_{\mathrm{T}}<0.08$ & $E_{\mathrm{T}}^{\text {cone }}(0.3) / E_{\mathrm{T}}<0.24$ \\
$>20$ & $p_{\mathrm{T}}^{\text {cone }}(0.3) / E_{\mathrm{T}}<0.10$ & $E_{\mathrm{T}}^{\text {cone }}(0.3) / E_{\mathrm{T}}<0.28$ \\
\hline
\end{tabular}

Table 4.2: Tracker and calorimeter isolation criteria for electrons. The cuts were chosen to optimise the sensitivity of a low mass $H \rightarrow W W \rightarrow \ell \nu \ell \nu$ search.

Additionally, electrons with conversion vertices or without a hit in the first pixel layer are vetoed (to suppress the $W \gamma$ and $W+$ jet backgrounds).

For $E_{\mathrm{T}}<25 \mathrm{GeV}$ the $W+$ jet and QCD backgrounds are much larger. Thus a multivariate very tight likelihood identification is used, with similar signal efficiency to the cut-based tight criteria but improved background rejection. It uses the signal and background probability density functions of multiple input variables to construct a likelihood discriminant, which may be cut upon (effectively choosing an operating point). The input variables are similar to those used in medium identification.

\section{Isolation}

Rejection of hadronic fakes or electrons from heavy flavour decays is further improved by requiring the electron to be isolated from activity in the tracker and calorimeter. The cuts are summarised in Table 4.2 and explained below.

Tracker isolation: $p_{\mathrm{T}}^{\text {cone }}\left(R_{0}\right)$ is the summed $p_{\mathrm{T}}$ of all tracks of $p_{\mathrm{T}}>0.4 \mathrm{GeV}$ within a cone of $\Delta R<R_{0}$, excluding the electron track itself. For robustness against pile-up, the tracks are required to originate from the primary vertex.

Calorimeter isolation: Uncalibrated topological clusters (see Section 4.2.5) are built from energy deposits in the ECal and HCal. These are more robust against pile-up than cell deposits as they suppress noise. $E_{\mathrm{T}}^{\text {cone }}\left(R_{0}\right)$ is the summed $E_{\mathrm{T}}$ of the topological clusters within a cone of $\Delta R<R_{0}$, excluding cells in a $5 \times 7$ window around the electron (see Figure 4.2). Corrections are made for electron energy leakage and deposits from pile-up and the underlying event.

\section{Quality}

An electron is rejected if its cluster is affected by a localised detector problem (e.g. a dead cell). 


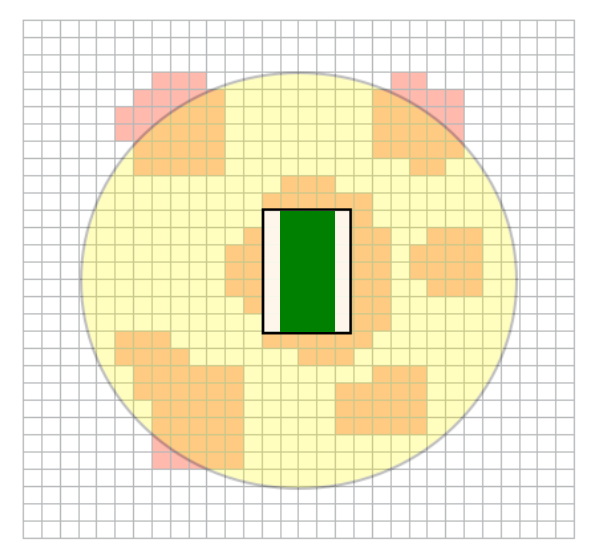

Figure 4.2: Schematic of calorimeter isolation, adapted from reference [106]. Pink cells constitute topological clusters, the yellow circle is the isolation cone $\left(R_{0}=0.4\right.$ shown), the green $3 \times 7$ rectangle is the reconstructed electron cluster, and the white $5 \times 7$ rectangle is the area removed from the cone.

\section{Primary vertex association}

To associate the electron with the primary vertex, the transverse impact parameter $d_{0}$ is required to be within three standard deviations of zero. Also, the longitudinal impact parameter $z_{0}$ is constrained by $\left|z_{0} \sin \theta\right|<0.4 \mathrm{~mm}$.

\section{Efficiency}

The efficiency of each selection step (reconstruction, identification, isolation and primary vertex association) is measured via tag-and-probe of $Z \rightarrow$ ee events $[105,107]$. This involves selecting an unbiased sample of events containing a well-identified tag object and a loosely-identified probe object, and measuring the selection efficiency of the probes. The sample must be clean (often enforced by a mass constraint) and backgrounds estimated (usually with a side-bands or template fit).

The tag is a well-identified electron, the probe is an electron passing the previous step (or an ECal cluster) and the constraint is $80 \mathrm{GeV}<m_{\text {(tag, probe) }}<100 \mathrm{GeV}$. The reconstruction and identification efficiencies are shown in Figures 4.3 and 4.4 respectively. Comparison with MC yields efficiency scale factors. 

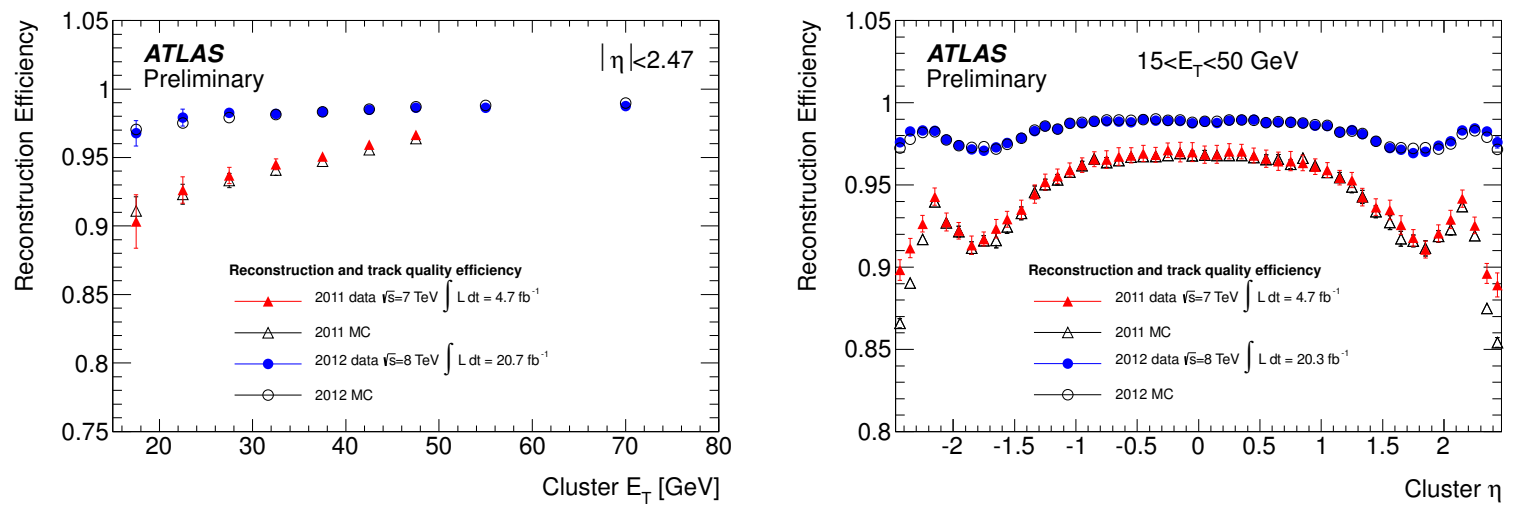

Figure 4.3: Electron reconstruction efficiency versus $E_{\mathrm{T}}$ (left) and $\eta$ (right), measured using tag-and-probe of $Z \rightarrow e e$ data and compared to MC [107]. In 2012, the implementation of GSF tracking gave significant performance improvement.
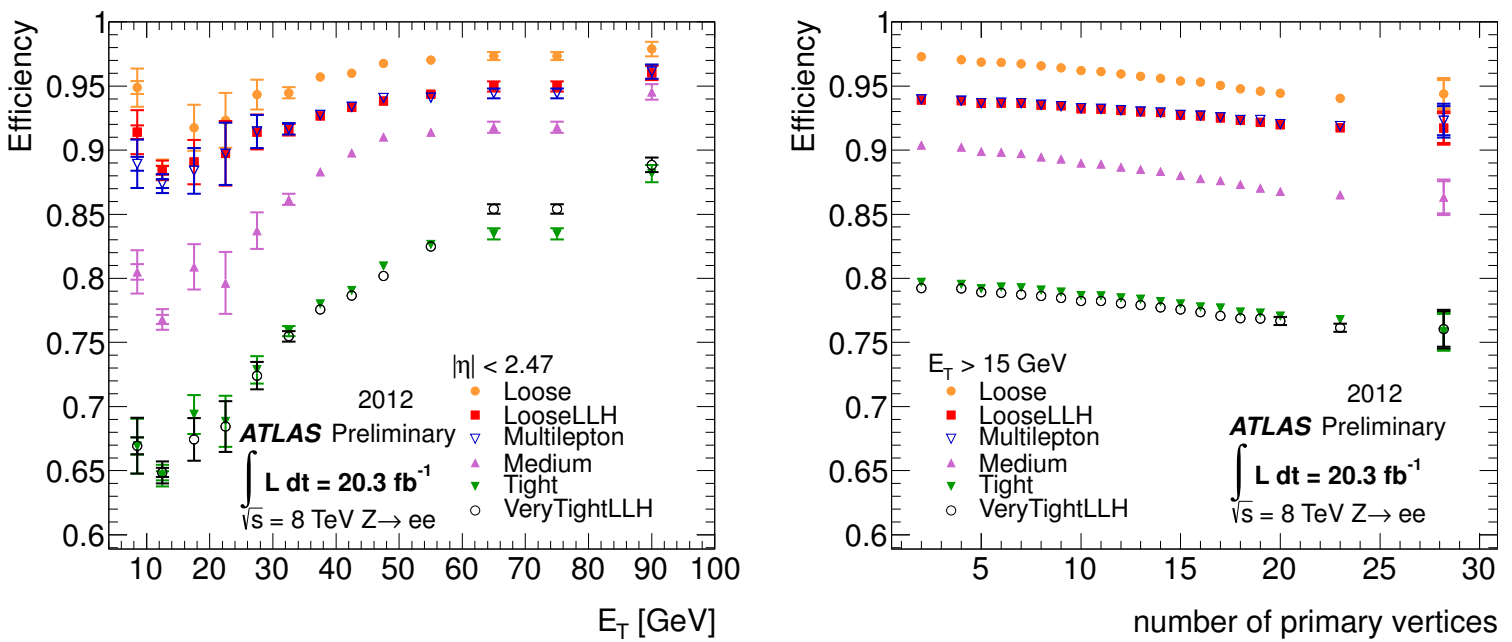

Figure 4.4: Efficiency of a variety of electron identification operating points versus $E_{\mathrm{T}}$ (left) and $N_{\mathrm{PV}}$ (right), measured using tag-and-probe of $Z \rightarrow e e$ data [107]. 'Very tight likelihood' has similar efficiency to 'tight', but improves background rejection. All operating points are fairly robust against pile-up. 'Multilepton' is an operating point developed for multilepton searches, and uses observables related to bremsstrahlung. 


\subsubsection{Muons}

Muons will pass through the ID, deposit minimal energy in the calorimeters and continue through the muon spectrometer (MS). Thus, they can be found via activity in the MS, optionally matched to an ID track. Muons with $p_{\mathrm{T}}>10 \mathrm{GeV}$ and $|\eta|<2.5$ are selected.

\section{Reconstruction}

Muons are found by matching MS tracks to ID tracks ${ }^{3}$ and their momenta are measured from a combination of the track curvatures [100].

First, the Muonboy algorithm [108] identifies a $\Delta \eta \times \Delta \phi \approx 0.4 \times 0.4$ region of activity via the muon trigger chambers (see Section 3.3.3), and then reconstructs localised track segments from nearby MDT and CSC hits. Segments in different layers are combined to form a track. Finally, a global track fit using the full MS hit information is performed, accounting for energy loss.

MS tracks are extrapolated to the primary vertex, accounting for energy loss in the calorimeter, and matched to ID tracks (see Section 4.2.1). The statistical combination of the two tracks is performed by the STACO algorithm [108], which weights each track by its covariance matrix. The muon four-momentum is determined from this combined track (dominated by the ID track at low- $p_{\mathrm{T}}$ and by the MS track at high- $\left.p_{\mathrm{T}}\right)$, and is calibrated with $Z \rightarrow \mu \mu$ events [109].

\section{Isolation}

Although the muon signature is much cleaner than that of electrons, isolation criteria are still required to suppress muons from hadronic decays and misidentified hadrons exiting the calorimeter (punch-through or sail-through). These criteria are summarised in Table 4.3. The $p_{\mathrm{T}}^{\text {cone }}\left(R_{0}\right)$ and $E_{\mathrm{T}}^{\text {cone }}\left(R_{0}\right)$ variables are defined similarly to electrons, though the size of the subtracted calorimeter window is reduced for muons.

\footnotetext{
${ }^{3}$ A variety of muon reconstruction strategies are available: stand-alone muons have only MS tracks, combined muons have both MS and ID tracks, segment-tagged muons have ID tracks that match to an MS track segment, and calorimeter-tagged muons have ID tracks matched to the calorimeter deposit of a minimum ionising particle (used to recover efficiency at $\eta \approx 0$, where detector services reduce the MS coverage). This thesis uses combined muons, which offer the best performance.
} 


\begin{tabular}{cll}
\hline Muon $p_{\mathrm{T}}(\mathrm{GeV})$ & Tracker isolation & Calorimeter isolation \\
\hline $10-15$ & $p_{\mathrm{T}}^{\text {cone }}(0.4) / p_{\mathrm{T}}<0.06$ & $E_{\mathrm{T}}^{\text {cone }}(0.3) / p_{\mathrm{T}}<0.06$ \\
$15-20$ & $p_{\mathrm{T}}^{\text {cone }}(0.3) / p_{\mathrm{T}}<0.08$ & $E_{\mathrm{T}}^{\text {cone }}(0.3) / p_{\mathrm{T}}<0.12$ \\
$20-25$ & $p_{\mathrm{T}}^{\text {cone }}(0.3) / p_{\mathrm{T}}<0.12$ & $E_{\mathrm{T}}^{\text {cone }}(0.3) / p_{\mathrm{T}}<0.18$ \\
$>25$ & $p_{\mathrm{T}}^{\text {cone }}(0.3) / p_{\mathrm{T}}<0.12$ & $E_{\mathrm{T}}^{\text {cone }}(0.3) / p_{\mathrm{T}}<0.30$ \\
\hline
\end{tabular}

Table 4.3: Tracker and calorimeter isolation criteria for muons. The cuts were chosen to optimise the sensitivity of a low mass $H \rightarrow W W \rightarrow \ell \nu \ell \nu$ search.

\section{Quality}

The ID track must satisfy quality criteria on the number of traversed sensors:

- $n_{\text {pixel }}^{\text {hit }}+n_{\text {pixel }}^{\text {dead }} \geq 1$

- $n_{\mathrm{SCT}}^{\mathrm{hit}}+n_{\mathrm{SCT}}^{\text {dead }} \geq 5$

- $n_{\text {pixel }}^{\text {hole }}+n_{\mathrm{SCT}}^{\text {hole }} \leq 2$

- $n_{\mathrm{TRT}}^{\text {hit }}+n_{\mathrm{TRT}}^{\text {outlier }} \geq 6$ and, for $|\eta|<1.9$, at least $10 \%$ are hits

where a hole is an expected hit that is not observed (excluding dead modules).

\section{Primary vertex association}

To associate the muon with the primary vertex, the transverse impact parameter $d_{0}$ is required to be within three standard deviations of zero. Also, the longitudinal impact parameter $z_{0}$ is constrained by $\left|z_{0} \sin \theta\right|<1.0 \mathrm{~mm}$.

\section{Efficiency}

The efficiency of each selection step is measured via tag-and-probe (see Section 4.2.3) of $Z \rightarrow \mu \mu$ events [109]. For example, the reconstruction efficiency is shown in Figure 4.5. Comparison with MC yields efficiency scale factors.

\subsubsection{Jets}

A hadron will pass through the ID (leaving hits if charged) before being absorbed by the ECal and HCal. The finite resolution of the calorimeter and the large number of overlapping hadronic showers make it impossible to reconstruct individual hadrons. For this reason, and more theoretical ones outlined in Section 2.3, jets are reconstructed from energy deposits. The main challenge in identifying jets originating from the hard scatter is calorimeter noise resulting from the electronics and pile-up. 

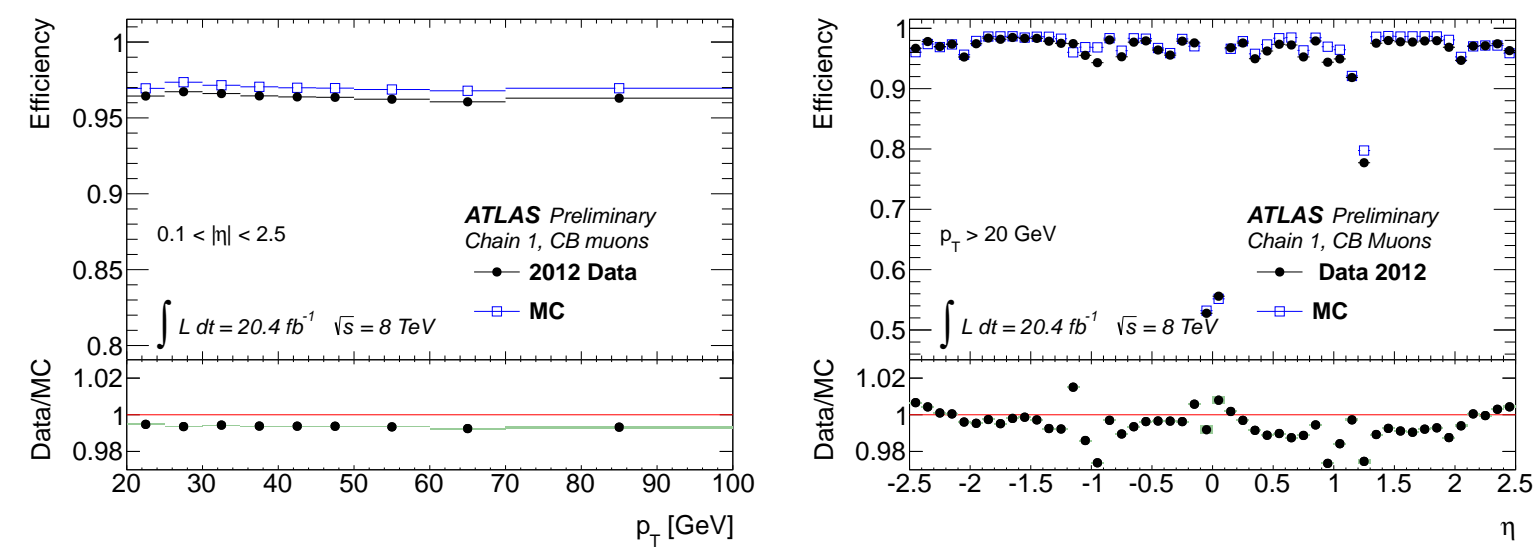

Figure 4.5: Muon reconstruction efficiency versus $p_{\mathrm{T}}$ (left) and $\eta$ (right), measured using tagand-probe of $Z \rightarrow \mu \mu$ data and compared to MC [109]. Reductions in efficiency at $\eta \approx 0$ and $\eta \approx 1.2$ are due to partial MS coverage (detector services and incomplete installation respectively). 'Chain 1' refers to STACO reconstruction.

Jets are selected with $p_{\mathrm{T}}>25 \mathrm{GeV}$ for central jets $(|\eta|<2.4)$ and with $p_{\mathrm{T}}>30 \mathrm{GeV}$ for forward jets $(2.4<|\eta|<4.5)$. However, occasionally it is useful to use jets with other $p_{\mathrm{T}}$ thresholds (e.g. the central jet veto in the VBF search). It shall be clearly stated when this is the case.

\section{Reconstruction}

To suppress contributions from calorimeter noise, energy deposits are grouped into topological clusters (topo-clusters) $[110,111]$. First, a seed cell is identified with signal-to-noise ratio $S / N>4$. Surrounding cells with $S / N>2$ are iteratively added. Finally, a single iteration of neighbouring cells with no $S / N$ criterion are added. When localised maxima exist within a cluster, it may be split. Clearly, the robustness to noise is determined by the choice of $N$, which is taken to be the sum in quadrature of the electronic noise and the pile-up noise expected with $\mu=30$.

The non-compensating calorimeter design yields different responses to electromagnetic and hadronic showers. ${ }^{4}$ Topo-clusters are reconstructed with an assumed response of $\approx 1$ (EM scale) and should be corrected for average energy loss in nuclear interactions (jet energy scale or JES). An intermediate local cluster weighting (LCW) classifies each topo-cluster as electromagnetic or hadronic (via energy density and shower depth) and applies an appropriate energy correction.

\footnotetext{
${ }^{4}$ Significant hadronic shower energy is lost through slow neutrons, nuclear excitations and neutrinos.
} 

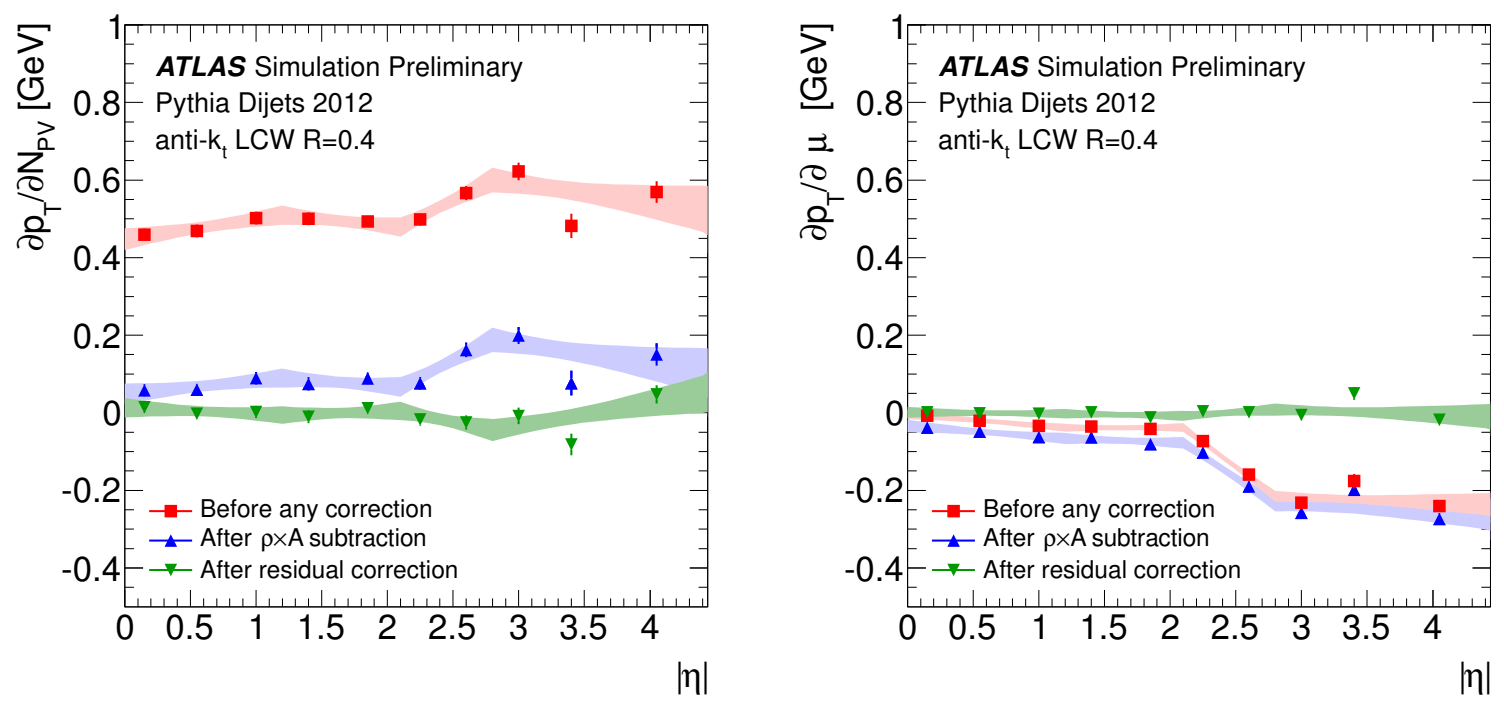

Figure 4.6: Dependence of jet $p_{\mathrm{T}}$ on in-time pile-up (left) and out-of-time pile-up (right), versus $|\eta|[112]$. The bands show the fits used in the correction.

These topo-clusters are then input to the anti- $k_{\mathrm{T}}$ jet algorithm with $R=0.4$ (see Section 2.3), under the assumption that the topo-clusters are massless.

\section{Calibration}

There are four steps to the jet calibration [111], described below.

First, the jet direction is adjusted such that it originates from the primary vertex, rather than the centre of the ATLAS coordinate system (a remnant of the jet algorithm).

Second, energy contributed by pile-up is subtracted [112]. This involves measuring the jet area $A$ (its susceptibility to additional particles with infinitesimal $p_{\mathrm{T}}$ ) and the pile-up density $\rho$ of the event (the median $p_{\mathrm{T}} / A$ of all $k_{\mathrm{T}}$ jets with $R=0.4$ ). Residual $|\eta|$-dependent corrections for in-time pile-up, $\alpha\left(N_{\mathrm{PV}}-1\right)$, and out-of-time pile-up, $\beta(\mu)$, are also made. Thus, the correction is

$$
p_{\mathrm{T}}^{\text {corr }}=p_{\mathrm{T}}-\rho A-\alpha\left(N_{\mathrm{PV}}-1\right)-\beta(\mu)
$$

and the improved robustness to pile-up that it provides is shown in Figure 4.6.

Third, the energy is calibrated from the LCW scale to the JES. The correction is derived from $\mathrm{MC}$ by comparing the energy of a reconstructed jet to that of the corresponding hadron-level jet (i.e. before detector simulation). 


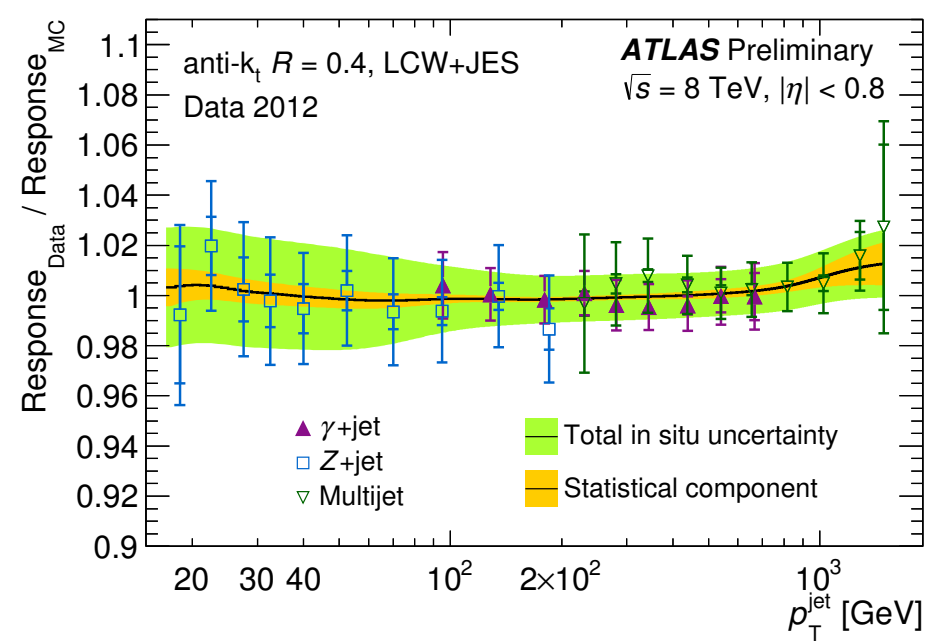

Figure 4.7: The ratio of the jet response measured in data to the MC prediction versus jet $p_{\mathrm{T}}$ [113]. This is the inverse of the in situ JES correction (see text). The results of three $p_{\mathrm{T}}$ balance studies and their combination (black line) are shown.

Finally, a residual in situ JES calibration corrects for mismodelling, and is applied to jets in data events only. The calibration exploits the $p_{\mathrm{T}}$ balance between a jet and a well-measured reference object, deriving the correction as the double-ratio

$$
\left\langle p_{\mathrm{T}}^{\mathrm{jet}} / p_{\mathrm{T}}^{\mathrm{ref}}\right\rangle_{\mathrm{MC}} /\left\langle p_{\mathrm{T}}^{\mathrm{jet}} / p_{\mathrm{T}}^{\mathrm{ref}}\right\rangle_{\mathrm{data}}
$$

The reference object is chosen to be a $Z$ boson (decaying to $e^{+} e^{-}$or $\mu^{+} \mu^{-}$) at low $p_{\mathrm{T}}$, a photon at medium $p_{\mathrm{T}}$, or a system of well-calibrated low- $p_{\mathrm{T}}$ jets at high $p_{\mathrm{T}}$. The contribution of each technique to the correction is shown in Figure 4.7.

\section{Quality}

Quality criteria (known as looser) reject calorimeter noise based on pulse shapes [114]. The selection is more than $99.8 \%$ efficient for jets with $p_{\mathrm{T}}>20 \mathrm{GeV}$.

\section{Primary vertex association}

The jet vertex fraction (JVF) is defined as the fraction of associated tracks originating from the primary vertex $\left(\left|z_{0} \sin \theta\right|<1 \mathrm{~mm}\right)$, weighted by track $p_{\mathrm{T}}$ [112]. It is a powerful discriminant against pile-up jets, but is only available for $|\eta|<2.4$.

Central jets with $p_{\mathrm{T}}<50 \mathrm{GeV}$ are required to have either JVF $>0.5$ or zero associated tracks. The efficiency of this cut is measured in situ with $Z+$ jet events. 


\subsection{6 b-jets}

A $b$-tagging algorithm exploits the long lifetimes of $b$-hadrons to identify jets originating from $b$-quarks. This aids suppression of top backgrounds, since $\operatorname{BR}(t \rightarrow W b) \approx 100 \%$. The MV1 algorithm uses a neural network with inputs of jet $p_{\mathrm{T}}$ and $\eta$, and the weights output by three other algorithms [115]:

- IP3D identifies jet tracks with significant impact parameters,

- SV1 searches for secondary vertices associated with b-hadron decays,

- JetFitter reconstructs the decay chain topology (including the daughter $c$-hadron).

An operating point is chosen to tag $85 \%$ of $b$-jets, with a mis-tag rate of $\sim 40 \%$ for $c$-jets, $\sim 35 \%$ for $\tau$-jets and $\sim 10 \%$ for light jets (initiated by $u, d, s$ or $g$ ). Note that $b$-tagging is only available for jets with $|\eta|<2.5$, since it relies upon tracks.

Modelling $b$-tagging algorithms is difficult, partly due to limited knowledge of $b$ hadron decays; thus it is imperative to measure their efficiency with experimental data. This was done with dileptonic $t \bar{t}$ decays, using a combinatorial likelihood method [116]. Comparison with MC yields efficiency scale factors.

\subsubsection{Missing transverse momentum}

The near-hermetic detector design enables information of non-interacting particles (e.g. neutrinos) to be inferred from measurements of interacting particles. Since the initial state has negligible nett $\boldsymbol{p}_{\mathrm{T}}$, momentum conservation implies that the visible and invisible $\boldsymbol{p}_{\mathrm{T}}$ in the final state must balance. Thus, the negative sum of the visible $\boldsymbol{p}_{\mathrm{T}}$ is known as missing transverse momentum, and equals the sum of the invisible $\boldsymbol{p}_{\mathrm{T}}$. Its magnitude is referred to as $p_{\mathrm{T}}^{\text {inv }}$, though alternative symbols are used depending upon its experimental reconstruction (see below).

Since the measurement of $p_{\mathrm{T}}^{\text {inv }}$ relies on the measurement of all the visible particles produced in the event, it generally has a poor experimental resolution. In particular, pile-up has an adverse effect on performance (see Figure 4.8). Object mismeasurement can produce a fake imbalance that is highly correlated to the event kinematics.

\section{Calorimeter-based $\mathrm{E}_{\mathrm{T}}^{\mathrm{miss}}$}

One can reconstruct missing transverse momentum with calorimeter energy deposits [117]. This is denoted $\boldsymbol{E}_{\mathrm{T}}^{\text {miss }}$, with magnitude $E_{\mathrm{T}}^{\text {miss }}$, since it is calorimeter-based. 

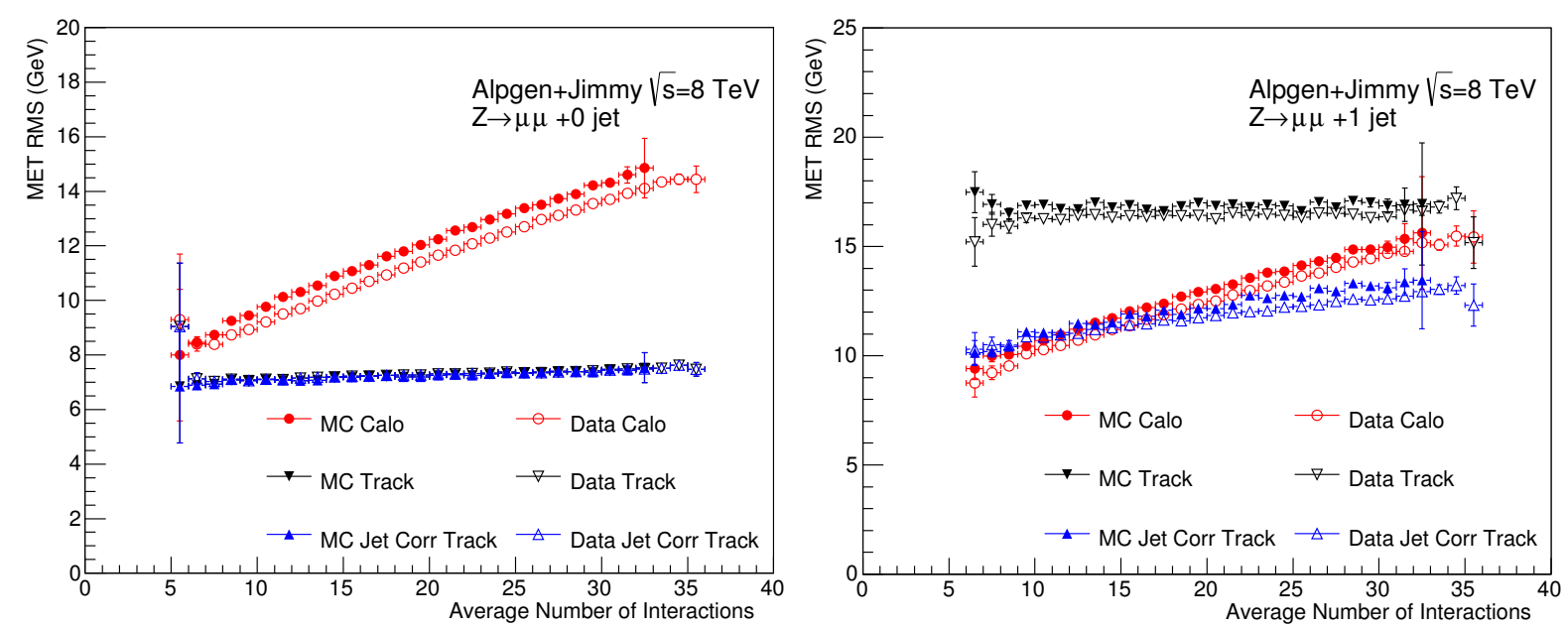

Figure 4.8: The root-mean-square of the missing transverse momentum versus the mean number of interactions per bunch crossing, $\mu$, measured in $Z \rightarrow \mu \mu$ events featuring 0 jets (left) and 1 jet (right). Results are shown for calorimeter-based $E_{\mathrm{T}}^{\text {miss }}\left(\right.$ red), track-based $p_{\mathrm{T}}^{\text {miss }}$ (black) and jet-corrected track-based $p_{\mathrm{T}}^{\text {miss,corr }}$ (blue). In the 0 -jet case, $p_{\mathrm{T}}^{\text {miss }}$ and $p_{\mathrm{T}}^{\text {miss,corr }}$ are identical.

First, calorimeter cells are associated with, and replaced by, calibrated high- $p_{\mathrm{T}}$ objects. To avoid double counting, the association is done in a specific order: electrons, photons [118], hadronically decaying $\tau$-leptons [119], jets and muons. For this measurement, some object selection criteria are loosened with respect to the preceding descriptions. Importantly, the JVF criterion is removed from jets and their $p_{\mathrm{T}}$ threshold is lowered to $20 \mathrm{GeV}$. Also, segment-tagged muons are used in addition to combined muons.

The low- $p_{\mathrm{T}}$ component is reconstructed using topo-clusters of the remaining calorimeter deposits, which are calibrated to the LCW scale (see Section 4.2.5). This is further improved by including unassociated tracks with $p_{\mathrm{T}}>400 \mathrm{MeV}$.

Thus, the calorimeter-based version of $p_{\mathrm{T}}^{\mathrm{inv}}$ is defined as

$$
\boldsymbol{E}_{\mathrm{T}}^{\mathrm{miss}}=-\left\{\sum_{e} \boldsymbol{p}_{\mathrm{T}}+\sum_{\mu} \boldsymbol{p}_{\mathrm{T}}+\sum_{\tau} \boldsymbol{p}_{\mathrm{T}}+\sum_{\gamma} \boldsymbol{p}_{\mathrm{T}}+\sum_{\text {jets }} \boldsymbol{p}_{\mathrm{T}}+\sum_{\text {soft }} \boldsymbol{p}_{\mathrm{T}}\right\} .
$$

Unfortunately, $E_{\mathrm{T}}^{\mathrm{miss}}$ is highly susceptible to pile-up because the starting points are calorimeter cells, which are not easily associated to the primary vertex. This is shown by the red points in Figure 4.8. 


\section{Track-based $\mathbf{p}_{\mathrm{T}}^{\text {miss }}$}

One can alternatively reconstruct missing transverse momentum with ID tracks. This is denoted $\boldsymbol{p}_{\mathrm{T}}^{\text {miss }}$, with magnitude $p_{\mathrm{T}}^{\text {miss }}$, since it is track-based.

Tracks with $p_{\mathrm{T}}>500 \mathrm{MeV}$ are selected if they have sufficiently small impact parameters with respect to the primary vertex $\left(\left|d_{0}\right|<1.5 \mathrm{~mm}\right.$ and $\left.\left|z_{0} \sin \theta\right|<1.5 \mathrm{~mm}\right)$. Quality criteria require $n_{\text {pixel }}^{\text {hit }} \geq 1$ and $n_{\mathrm{SCT}}^{\text {hit }} \geq 6$. Additional tracks may be used if they are associated with a lepton with $\left|z_{0} \sin \theta\right|<1.0 \mathrm{~mm}$ (the electron identification criteria are loosened to medium $c$.f. Section 4.2.3, and the muon $p_{\mathrm{T}}$ threshold is lowered to $6 \mathrm{GeV}$ c.f. Section 4.2.4).

Occasionally, tracks are poorly reconstructed, which can have a significant impact upon $p_{\mathrm{T}}^{\text {miss }}$. Therefore, tracks with $p_{\mathrm{T}}>100 \mathrm{GeV}$ that are not associated to any physics object are removed from the selection. Similarly, a track within $\Delta R<0.4$ of a jet with $p_{\mathrm{T}}>10 \mathrm{GeV}$ is removed if $p_{\mathrm{T}}^{\text {track }}>1.4 p_{\mathrm{T}}^{\text {jet }}$.

Bremsstrahlung from an electron can convert to $e^{+} e^{-}$pairs, resulting in additional tracks. For this reason, tracks within a cone of $\Delta R<0.05$ of a reconstructed electron are collectively replaced with the calibrated electron $p_{\mathrm{T}}$. Muon ID tracks are also replaced by the $p_{\mathrm{T}}$ of the reconstructed muon.

Thus, the track-based version of $p_{\mathrm{T}}^{\text {inv }}$ is defined as

$$
\boldsymbol{p}_{\mathrm{T}}^{\mathrm{miss}}=-\left\{\sum_{e} \boldsymbol{p}_{\mathrm{T}}+\sum_{\mu} \boldsymbol{p}_{\mathrm{T}}+\sum_{\text {unassoc. }} \boldsymbol{p}_{\mathrm{T}}\right\} .
$$

$p_{\mathrm{T}}^{\text {miss }}$ is very resilient to pile-up. However, its inability to include neutral hadrons significantly degrades the resolution. This is shown by the black points in Figure 4.8.

\section{Jet-corrected track-based $\mathbf{p}_{\mathrm{T}}^{\text {miss,corr }}$}

It is possible to correct $p_{\mathrm{T}}^{\text {miss }}$ for neutral hadrons using calorimeter information. Tracks within $\Delta R<0.4$ of a jet are collectively replaced by the calibrated jet $p_{\mathrm{T}}$. Thus, the jet-corrected track-based version of $p_{\mathrm{T}}^{\mathrm{inv}}$ is defined as

$$
\boldsymbol{p}_{\mathrm{T}}^{\text {miss,corr }}=-\left\{\sum_{e} \boldsymbol{p}_{\mathrm{T}}+\sum_{\mu} \boldsymbol{p}_{\mathrm{T}}+\sum_{\text {jets }} \boldsymbol{p}_{\mathrm{T}}+\sum_{\text {unassoc. }} \boldsymbol{p}_{\mathrm{T}}\right\}
$$

This has the best resolution, but the use of calorimeter information does introduce some pile-up dependence. This is shown by the blue points in Figure 4.8. 


\section{Relative missing transverse momentum}

The effect of object mismeasurement upon missing transverse momentum can be reduced by considering the component transverse to the nearest reconstructed object. Thus, the relative $E_{\mathrm{T}}^{\text {miss }}$ is defined to be

$$
E_{\mathrm{T}, \mathrm{rel}}^{\mathrm{miss}}= \begin{cases}E_{\mathrm{T}}^{\mathrm{miss}} \sin (\Delta \phi) & \text { if } \Delta \phi<\pi / 2 \\ E_{\mathrm{T}}^{\mathrm{miss}} & \text { otherwise }\end{cases}
$$

where $\Delta \phi$ is the azimuthal angle between $\boldsymbol{E}_{\mathrm{T}}^{\mathrm{miss}}$ and the nearest electron, muon or jet object. Similar definitions exist for $p_{\mathrm{T}, \text { rel }}^{\text {miss }}$ and $p_{\mathrm{T}, \text { rel }}^{\text {miss,corr }}$.

\subsubsection{Object overlap removal}

In order to avoid double-counting calorimeter deposits and tracks as multiple physics objects, overlapping objects are removed according to the following rules:

- $\Delta R(e, \mu)<0.1 \quad$ remove electron

- $\Delta R(e, e)<0.1 \quad$ remove electron with lower $p_{\mathrm{T}}$

- $\Delta R(e, j)<0.3 \quad$ remove jet

- $\Delta R(\mu, j)<0.3 \quad$ remove muon

where $e, \mu$ and $j$ are the electron, muon and jet objects defined in Sections 4.2.3-4.2.5.

Additionally, events are rejected if a muon passing loose reconstruction (i.e. without isolation, quality, primary vertex and $p_{\mathrm{T}}$ criteria) is found within $\Delta R<0.05$ of an electron passing the full reconstruction. This is effective at rejecting rare $Z \rightarrow \mu \mu$ events where a muon radiates a hard photon, which subsequently converts and passes the electron reconstruction. 


\subsection{Event selection criteria}

Section 4.1 outlined the basic experimental signature of the search as two oppositely charged leptons and significant $p_{\mathrm{T}}^{\mathrm{inv}}$. Thus, the initial stages of the event selection are responsible for finding this signature. Subsequent criteria, or cuts, target specific background processes. Their aim is to improve the analysis sensitivity by suppressing backgrounds whilst retaining a sufficient number of signal events.

\subsubsection{Data quality}

The $p p$ dataset (see Section 3.2.2) is hierarchically split into periods of broadly consistent beam conditions, runs typically corresponding to LHC fills, and luminosity blocks of $\sim 2$ minutes where the instantaneous luminosity is approximately constant. Luminosity blocks are included in the analysis if the detector was operating sufficiently for the recorded data to be considered 'good for physics' (see Figure 3.2a). ${ }^{5}$ For the 2012 dataset, this corresponds to a total integrated luminosity of $20.3 \mathrm{fb}^{-1}$.

Individual events are also vetoed if certain data quality criteria are failed by:

- a noise burst in the LAr calorimeter,

- data corruption caused by a restart of the synchronisation system,

- a looser jet is reconstructed with $p_{\mathrm{T}}>20 \mathrm{GeV}$ (indicative of an HCal spike),

- a jet is reconstructed near a 'hot' HCal tile (1st - 8th May 2012 only).

A further quality criterion requires that the primary vertex considered as the hard scatter (that with the highest $\sum p_{\mathrm{T}}^{2}$ ) must be associated with at least three tracks. This reduces the cosmic ray background to negligible levels.

\subsubsection{Trigger}

It is infeasible to record all the delivered collisions; ATLAS employs a trigger system to identify and record interesting events (see Section 3.3.4). In the $H \rightarrow W W \rightarrow \ell \nu \ell \nu$ search it is natural to trigger on high- $p_{\mathrm{T}}$ leptons, using algorithms similar to, though less sophisticated than, those in Sections 4.2.3 and 4.2.4.

\footnotetext{
${ }^{5}$ ATLAS good runs list: data12_8TeV.periodAllYear_DetStatus-v61-pro14-02_DQDefects-00-01 -00_PHYS_StandardGRL_All_Good.xml
} 

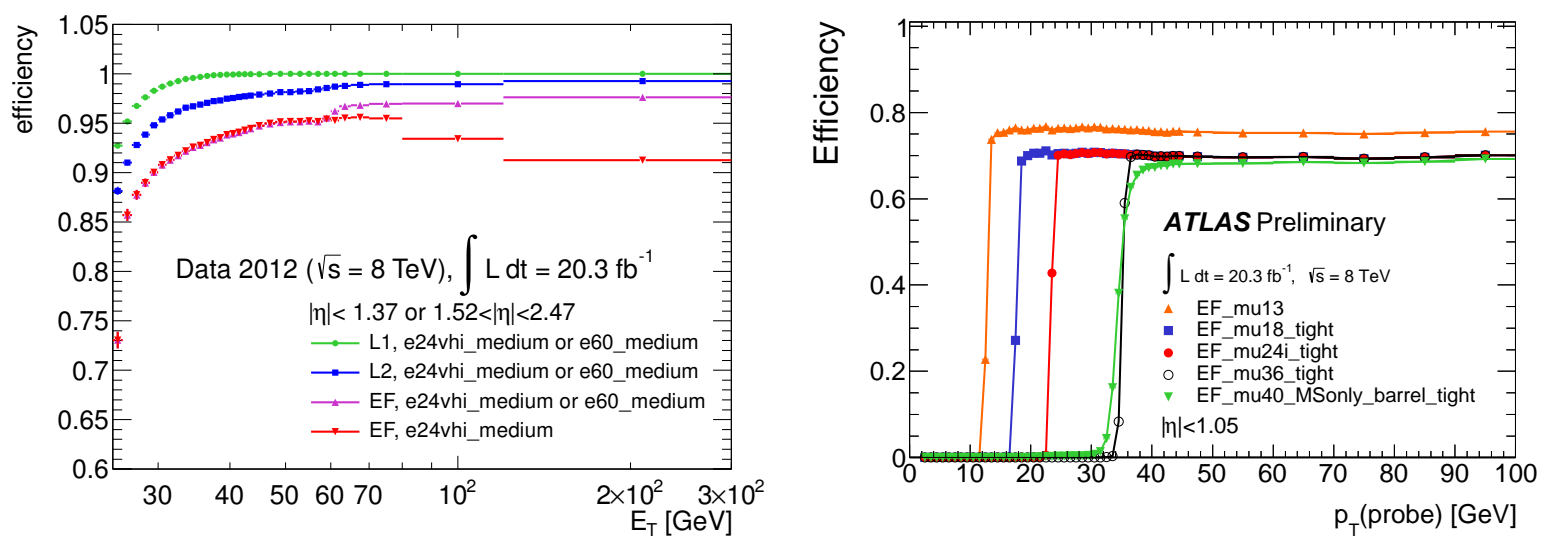

Figure 4.9: Efficiencies of the single-lepton triggers for electrons with respect to offline medium identification (left) and muons with respect to offline reconstruction (right) [120].

A trigger is characterised by its efficiency versus $p_{\mathrm{T}}$ curve (though it also depends on $\eta$ ), which has a turn-on region followed by a plateau, as shown in Figure 4.9. It is preferable to operate on the plateau, where the efficiency is more stable and has smaller uncertainty. To maximise the signal yield, it is desirable to use a trigger with a lower turn-on $p_{\mathrm{T}}$. However, increased backgrounds and limitations to trigger latency and bandwidth require a compromise to be found. The lowest unprescaled ${ }^{6}$ single-lepton triggers available in 2012 had nominal $p_{\mathrm{T}}$ thresholds of $24 \mathrm{GeV}$. Fortunately, it is possible to recover trigger efficiency at lower $p_{\mathrm{T}}$ by using dilepton triggers, because the backgrounds are much smaller when two leptons are required.

Events are required to pass at least one trigger listed in Table 4.4. The single-lepton triggers include a tighter low- $p_{\mathrm{T}}$ trigger and a looser high- $p_{\mathrm{T}}$ trigger in order to maximise the efficiency. Dilepton triggers are then used to recover some efficiency at lower $p_{\mathrm{T}}$. Together, these triggers support a dilepton signature with $p_{\mathrm{T}}$ thresholds of $22 \mathrm{GeV}$ and $10 \mathrm{GeV}$ in the offline analysis, whilst operating on the plateau.

Additionally, events are required to have at least one lepton passing the offline reconstruction that is matched within $\Delta R<0.15$ of a triggered lepton object. Singlelepton triggers are matched to offline leptons with $p_{\mathrm{T}}>25 \mathrm{GeV}$. Dilepton triggers comprise two of the following single-lepton triggers: mu8 is matched to offline muons with

\footnotetext{
${ }^{6}$ A prescaled trigger reduces the threshold $p_{\mathrm{T}}$ by recording only 1 in $N$ events passing the trigger, and weighting such events by a factor $N$. In doing so, statistical power is lost.
} 


\begin{tabular}{|c|c|c|c|}
\hline \multirow{2}{*}{$\begin{array}{l}\text { Single-lepton } \\
\text { triggers }\end{array}$} & $e$ & EF_e24vhi_medium1 or & EF_e60_medium1 \\
\hline & $\mu$ & EF_mu24i_tight & EF_mu36_tight \\
\hline \multirow{3}{*}{$\begin{array}{l}\text { Dilepton } \\
\text { triggers }\end{array}$} & $e e$ & EF_2e12Tvh_loose1 or & EF_2e12Tvh_loose1_L2StarB \\
\hline & $\mu \mu$ & EF_mu18_tight_mu8_EFFS & \\
\hline & $e \mu$ & EF_e12Tvh_medium1_mu8 & \\
\hline
\end{tabular}

Table 4.4: Employed triggers. EF refers to event filter, e is an electron, mu is a muon, the subsequent number is the $p_{\mathrm{T}}$ threshold, vh indicates calorimeter isolation, $i$ indicates track isolation, and tight, medium or loose is the identification. Other parts relate to the trigger chain. Criteria are looser than those applied offline.

$p_{\mathrm{T}}>10 \mathrm{GeV}$, mu18 is matched to offline muons with $p_{\mathrm{T}}>20 \mathrm{GeV}$, and e12 is matched to offline electrons with $p_{\mathrm{T}}>15 \mathrm{GeV}$. $^{7}$

Lepton trigger efficiencies are measured via tag-and-probe (see Section 4.2.3) of $Z \rightarrow \ell \ell$ events, where the tag and probe have both passed the offline lepton selection and the tag has successfully matched to a triggered lepton object. For example, single-lepton trigger efficiencies are displayed in Figure 4.9. Comparison with MC yields efficiency scale factors.

\subsubsection{Pre-selection of dilepton $+p_{\top}^{\text {inv }}$ signature}

Following the trigger selection, events are required to have two oppositely charged leptons passing the offline selection (see Section 4.2). The lepton with the highest $p_{\mathrm{T}}$, called the leading lepton, must have $p_{\mathrm{T}, \ell}^{\text {lead }}>22 \mathrm{GeV}$ and the subleading lepton must have $p_{\mathrm{T}, \ell}^{\text {sublead }}>10 \mathrm{GeV}$. Events containing a third lepton are vetoed in order to reject backgrounds with three or more leptons in the final state, such as $W Z$ production.

At this point, it is possible to split the dilepton final state into four channels according to the flavours of the two leptons: $e e, \mu \mu, e \mu, \mu e$ (where the first flavour is that of the leading lepton). This is very useful because the background compositions of the channels are dramatically different. For example, the $Z / \gamma^{*} \rightarrow \ell \ell$ background is much larger in the same flavour channels $(e e / \mu \mu)$ than the different flavour channels $(e \mu / \mu e)$.

\footnotetext{
${ }^{7}$ It follows that events featuring an electron with $p_{\mathrm{T}}<15 \mathrm{GeV}$ must fire a single-lepton trigger, and thus the leading lepton must have $p_{\mathrm{T}}>25 \mathrm{GeV}$.
} 

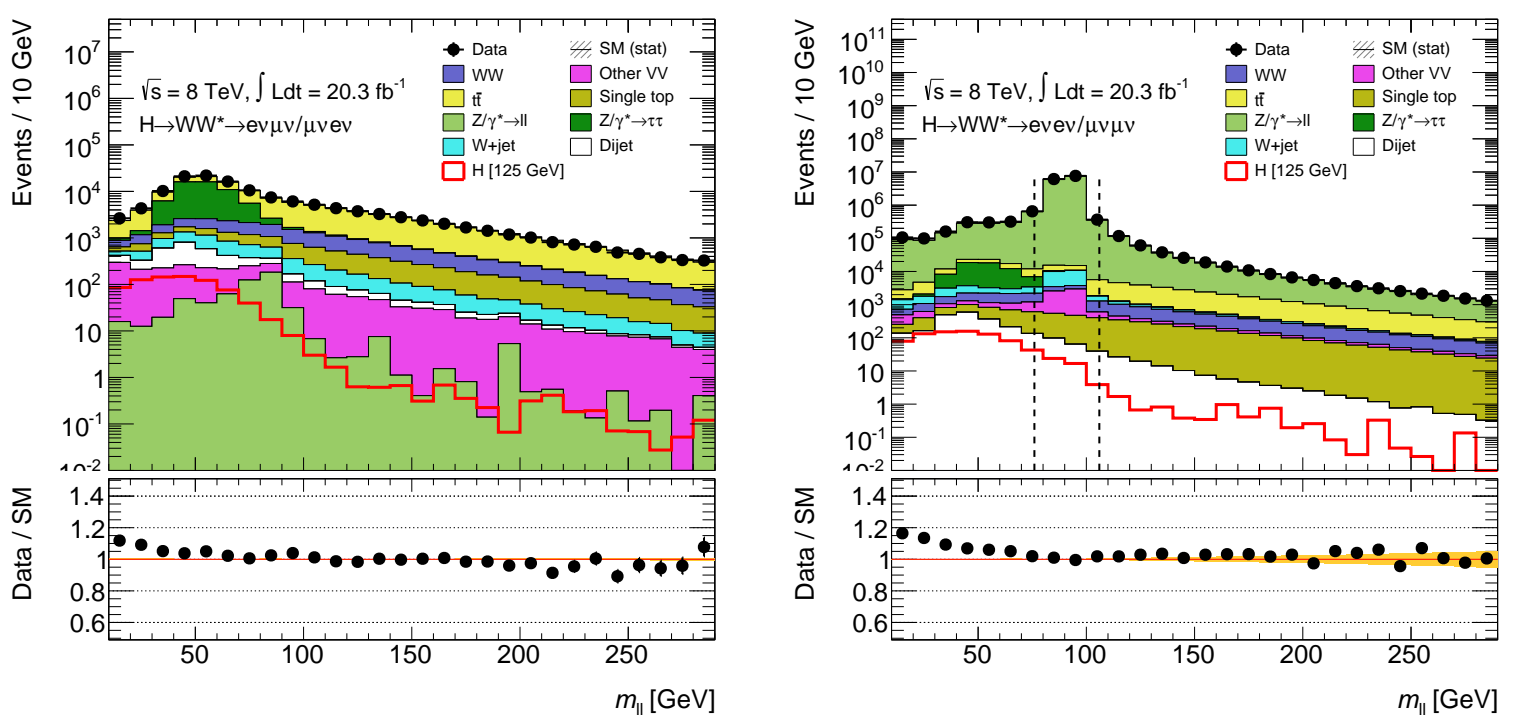

Figure 4.10: The $m_{\ell \ell}$ distribution in the $e \mu / \mu e$ (left) and $e e / \mu \mu$ (right) channels. These are made after the low $m_{\ell \ell}$ cut. The dashed lines enclose the $\left|m_{\ell \ell}-m_{Z}\right|>15 \mathrm{GeV}$ cut applied to the $e e / \mu \mu$ channels.

Low mass hadronic resonances with dileptonic decays (e.g. $J / \psi$ and $\Upsilon$ ) are removed from the $e e / \mu \mu$ channels by requiring the mass of the dilepton system $m_{\ell \ell}>12 \mathrm{GeV}$. This also greatly suppresses the low mass $Z / \gamma^{*}$ background. For the $e \mu / \mu e$ channels, a cut of $m_{\ell \ell}>10 \mathrm{GeV}$ suppresses leptons from heavy flavour decays and resonance decays to $\tau$ leptons. The $e e / \mu \mu$ channels are dominated by the $Z / \gamma^{*}$ background (see Figure 4.10), but most of these events can be rejected by vetoing a window around the $Z$ mass, $\left|m_{\ell \ell}-m_{Z}\right|>15 \mathrm{GeV}$, where $m_{Z}=91.1876 \mathrm{GeV}$.

Requiring significant $p_{\mathrm{T}}^{\text {inv }}$ suppresses the $Z / \gamma^{*} \rightarrow \ell \ell$ and dijet backgrounds (see Figure 4.11). It also suppresses the $Z / \gamma^{*} \rightarrow \tau \tau$ background as its neutrinos have a propensity to cancel in the $p_{\mathrm{T}}^{\mathrm{inv}}$ calculation. We require $p_{\mathrm{T}}^{\text {miss,corr }}>20 \mathrm{GeV}$ in the $e \mu / \mu e$ channels and $E_{\mathrm{T}, \mathrm{rel}}^{\text {miss }}>40 \mathrm{GeV}$ in the $e e / \mu \mu$ channels. In the $e \mu / \mu e$ channels, the $p_{\mathrm{T}}^{\text {inv }}$ cut is relaxed because the $Z / \gamma^{*} \rightarrow \ell \ell$ background is smaller.

Following the selection of dilepton events with significant $p_{\mathrm{T}}^{\mathrm{inv}}$, the $e \mu / \mu e$ channels are dominated by top background and the ee/ $\mu \mu$ channels are dominated by $Z / \gamma^{*} \rightarrow \ell \ell$. However, the background compositions of all channels are highly dependent upon the number of jets (see Figure 4.12). Thus, at this stage the analysis is binned according to jet multiplicity (0-jet, 1 -jet, $\geq 2$-jet), so that backgrounds can be targeted individually. In the $\geq 2$-jet bin, only the $e \mu / \mu$ e channels are used. 

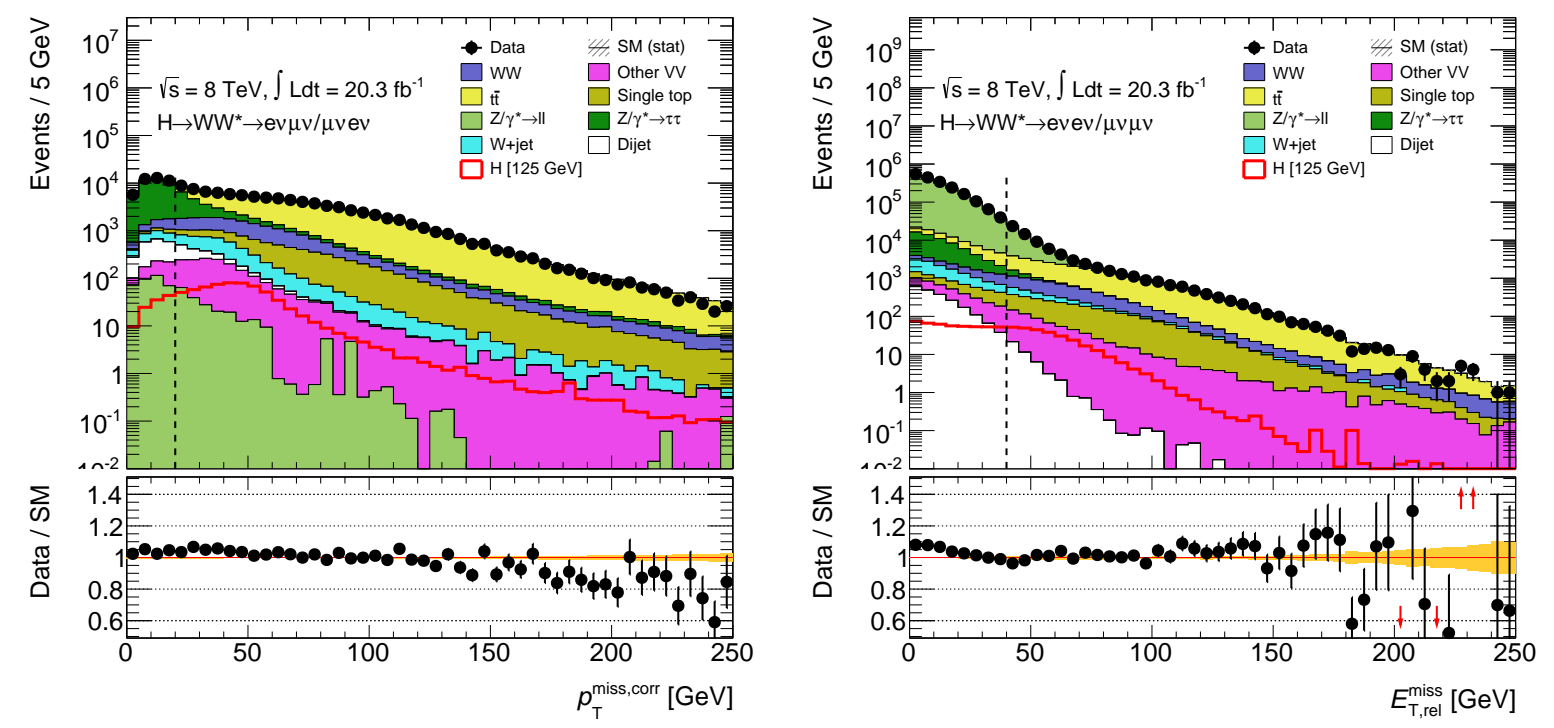

Figure 4.11: The $p_{T}^{\text {inv }}$ observable used in the selection of the $e \mu / \mu e$ (left) and $e e / \mu \mu$ (right) channels. These are made after the $Z$ mass veto cut. The selection removes events to the left of each dashed line.
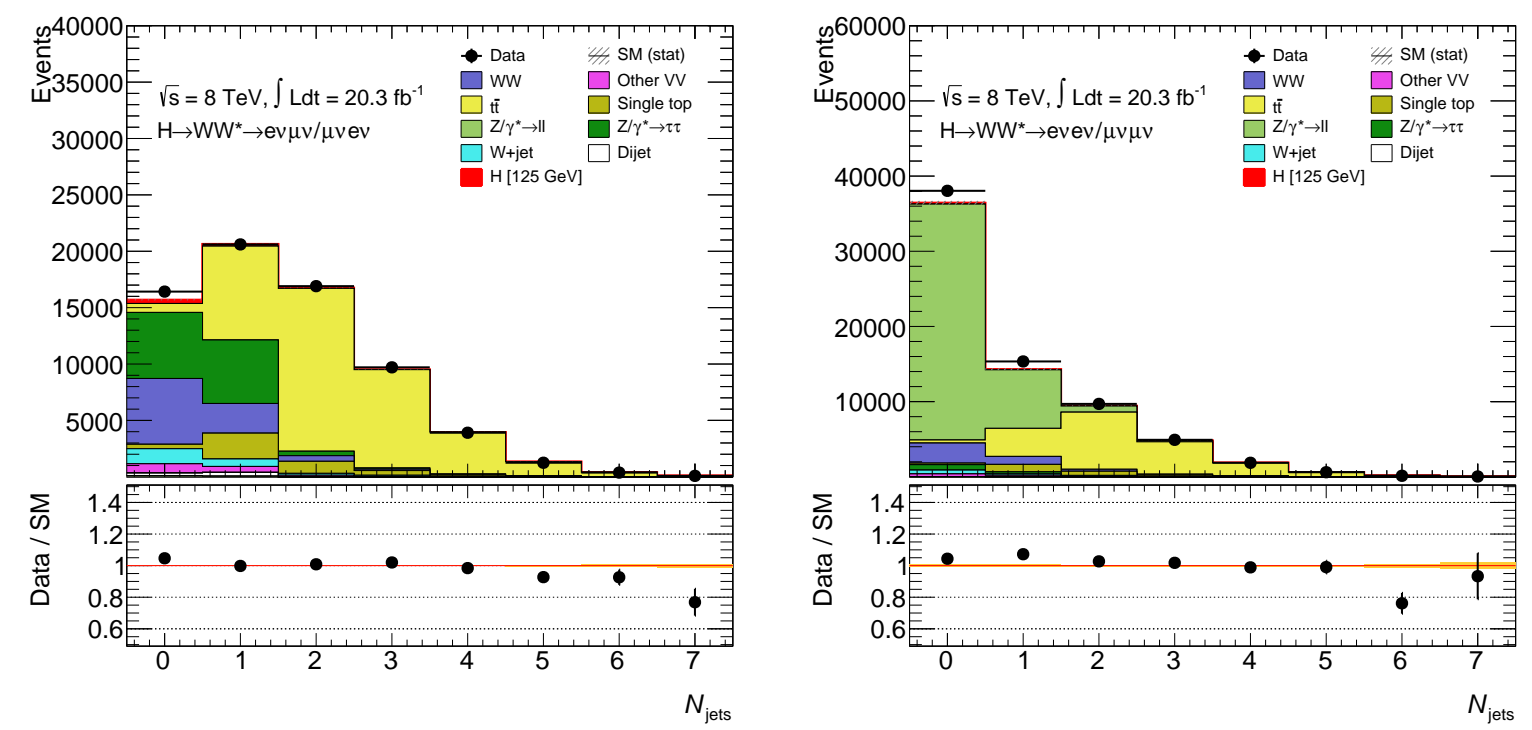

Figure 4.12: Jet multiplicity distribution in the $e \mu / \mu e$ (left) and $e e / \mu \mu$ (right) channels. These are made following the pre-selection. 


\subsection{4 $\mathrm{H} \rightarrow \mathrm{WW} \rightarrow \ell \nu \ell \nu$ decay topology}

The discrimination of $H \rightarrow W W$ signal from irreducible backgrounds that also feature a $W W$ pair is a problem common to all jet bins. Thus, the topology of the $H \rightarrow W W \rightarrow$ $\ell \nu \ell \nu$ decay is discussed before continuing with the event selection.

First, the spin-0 nature of the Higgs boson and the $\mathrm{V}-\mathrm{A}$ structure of the weak interaction (see Section 1.2) imply that a small opening angle between the two leptons is preferred. This follows from spin conservation and the extremely small masses of the neutrinos. Consequently, the mass of the dilepton system, $m_{\ell \ell}$, is also small. This follows from $m_{\ell \ell}^{2} \simeq 2 E_{\ell_{1}} E_{\ell_{2}}(1-\cos \vartheta)$, where $\vartheta$ is the opening angle. Thus, signal events are selected with the criteria $\Delta \phi(\ell, \ell)<1.8$ and $m_{\ell \ell}<55 \mathrm{GeV}$.

Second, the invariant mass of the dilepton + dineutrino system should correspond to $m_{H}$ (modified by a Breit-Wigner distribution). Unfortunately, as discussed above, it is only possible to infer the transverse component of the dineutrino system momentum. We therefore construct a transverse mass variable

$$
m_{\mathrm{T}}=\sqrt{\left(E_{\mathrm{T}, \ell \ell}+p_{\mathrm{T}}^{\text {miss,corr }}\right)^{2}-\left|\boldsymbol{p}_{\mathrm{T}, \ell \ell}+\boldsymbol{p}_{\mathrm{T}}^{\text {miss,corr }}\right|^{2}}
$$

where $E_{\mathrm{T}, \ell \ell}^{2}=p_{\mathrm{T}, \ell \ell}^{2}+m_{\ell \ell}^{2}$ and $\boldsymbol{p}_{\mathrm{T}}^{\text {miss,corr }}$ is used as it has the best resolution (see Section 4.2.7). At hadron-level this has an upper bound at $m_{H}$, though at detector-level the sharp cut-off is smeared by the poor $p_{\mathrm{T}}^{\text {miss,corr }}$ resolution. This $m_{\mathrm{T}}$ observable is used in the statistical fitting procedure.

\subsubsection{0 -jet selection}

The 0 -jet bin is dominated by $Z / \gamma^{*}$ and $W W$ backgrounds. A small number of pathological events where $\boldsymbol{p}_{\mathrm{T}}^{\text {miss,corr }}$ is near $\boldsymbol{p}_{\mathrm{T}, \ell \ell}$ are rejected by requiring $\Delta \phi\left(\ell \ell, p_{\mathrm{T}}^{\text {inv }}\right)>\pi / 2$.

Considering the $Z / \gamma^{*} \rightarrow \ell \ell$ background, the boson $p_{\mathrm{T}}$ will generally be less than the $p_{\mathrm{T}}$ threshold used in jet selection, since no jet has been found. Thus, the $Z / \gamma^{*}$ background is greatly reduced by requiring $p_{\mathrm{T}, \ell \ell}>30 \mathrm{GeV}$ (see Figure 4.13). In the $e e / \mu \mu$ channels only, the $Z / \gamma^{*} \rightarrow \ell \ell$ background is also suppressed by an additional cut $p_{\mathrm{T}, \mathrm{rel}}^{\text {miss }}>40 \mathrm{GeV}$ (see Figure 4.14).

After the signal topology selection (see Section 4.3.4), a large $Z / \gamma^{*} \rightarrow \ell \ell$ background remains in the $e e / \mu \mu$ channels, despite the significant $p_{\mathrm{T}}^{\text {inv }}$ requirement. This can be 

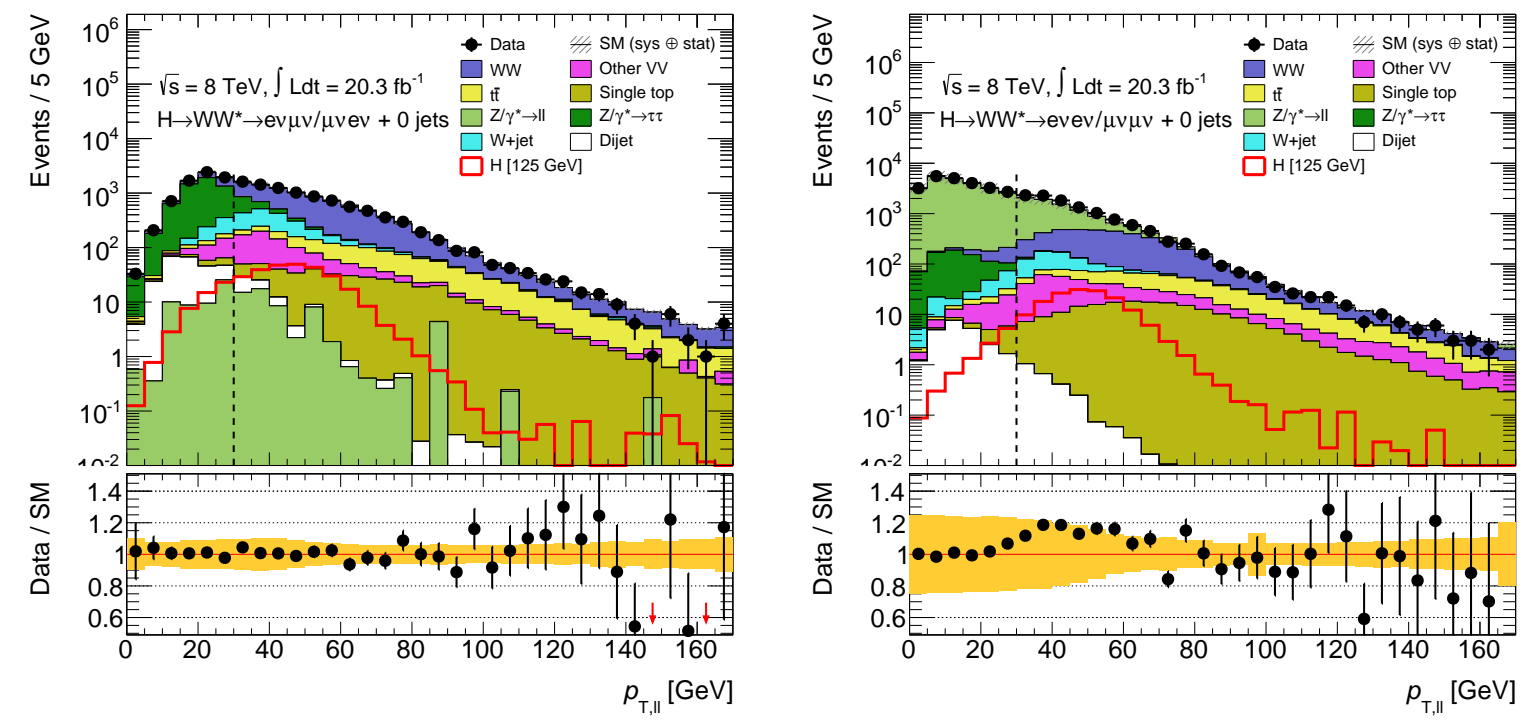

Figure 4.13: The $p_{\mathrm{T}, \ell \ell}$ distribution in the $e \mu / \mu e$ (left) and $e e / \mu \mu$ (right) channels. These are made in the 0 -jet bin, after the $\Delta \phi\left(\ell \ell, p_{\mathrm{T}}^{\mathrm{inv}}\right)$ cut. The selection removes events to the left of each dashed line.
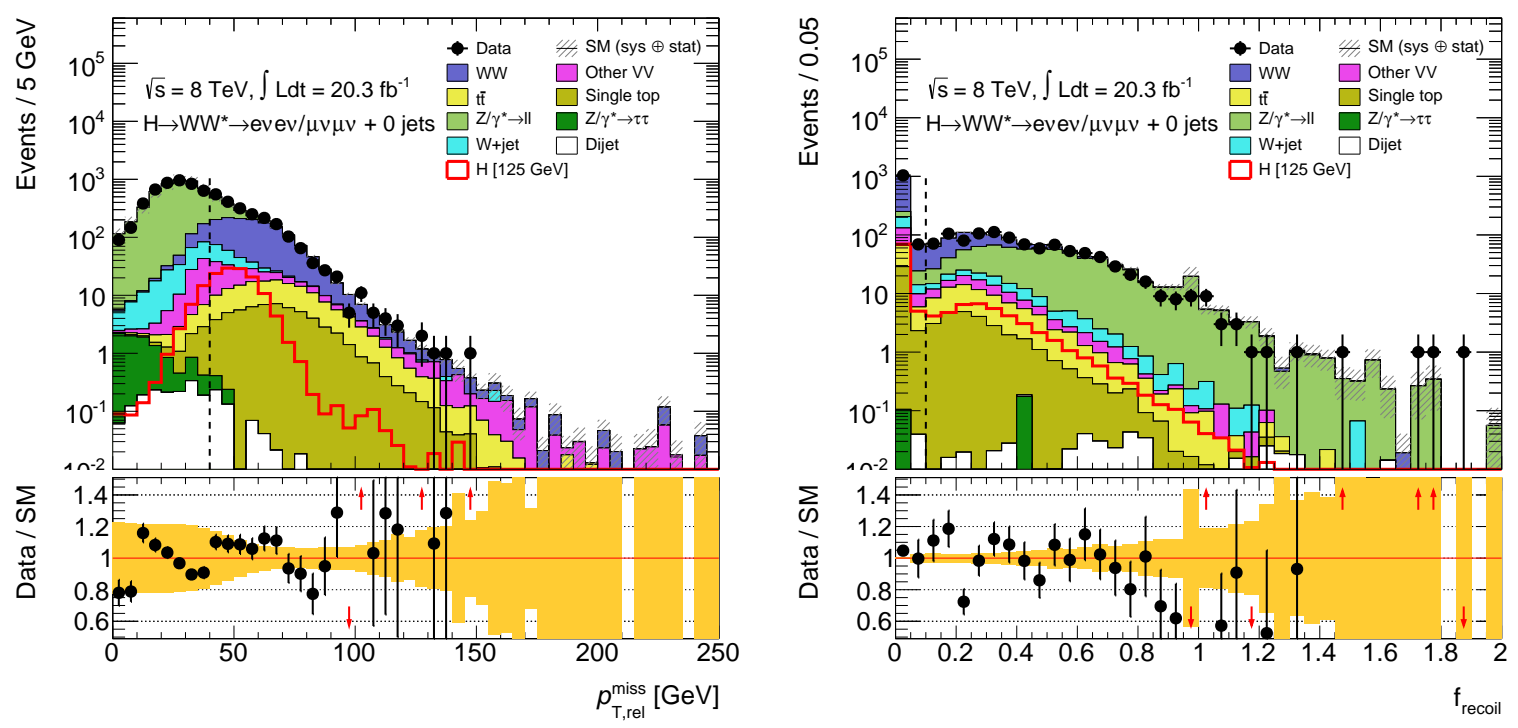

Figure 4.14: The $p_{\mathrm{T} \text {,rel }}^{\text {miss }}$ (left) and $f_{\text {recoil }}$ (right) distributions in the $e e / \mu \mu$ channels. The subsequent selection removes events to the left of the dashed line in the $p_{\mathrm{T} \text {,rel }}^{\text {miss }}$ plot, and removes events to the right of the dashed line in the $f_{\text {recoil }}$ plot. 

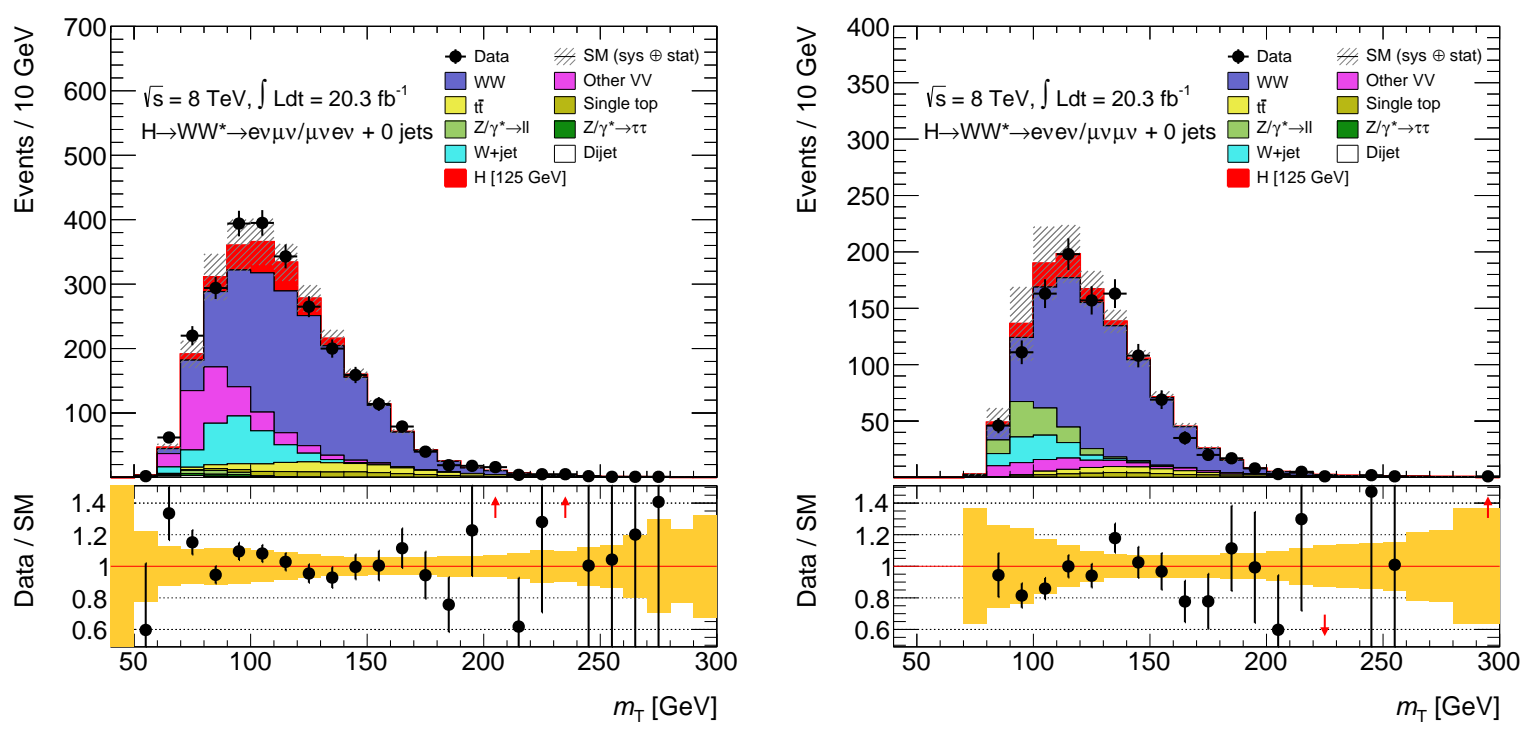

Figure 4.15: The $m_{\mathrm{T}}$ distribution of the selected 0-jet events, in the $e \mu / \mu e$ (left) and $e e / \mu \mu$ (right) channels.

further suppressed by searching for soft hadronic activity to balance the dilepton system. First, jets with $p_{\mathrm{T}}>10 \mathrm{GeV}$ and $|\eta|<4.5$ are found (as detailed in Section 4.2.5, minus the JVF cut). Then a discriminant is defined as

$$
f_{\text {recoil }}=\left|\sum_{j \text { in } \wedge} \mathrm{JVF}_{j} \cdot \boldsymbol{p}_{\mathrm{T}, j}\right| / p_{\mathrm{T}, \ell \ell}
$$

where $\wedge$ is the detector quadrant centred on $-\boldsymbol{p}_{\mathrm{T}, \ell \ell}$. This is essentially the fraction of $p_{\mathrm{T}, \ell \ell}$ that can be balanced by soft hadronic activity in the opposing quadrant, and so is larger in $Z / \gamma^{*} \rightarrow \ell \ell$ than in processes featuring prompt neutrinos. We require $f_{\text {recoil }}<0.1$ in the $e e / \mu \mu$ channels (see Figure 4.14). $f_{\text {recoil }}$ is also instrumental in estimating the $Z / \gamma^{*} \rightarrow \ell \ell$ background, and shall be revisited in Section 7.4.

The $m_{\mathrm{T}}$ distributions of the selected 0-jet events are shown in Figure 4.15. 


\subsubsection{1-jet selection}

The 1 -jet bin is initially dominated by the $Z / \gamma^{*}$ and top backgrounds (see Figure 4.12), though the top background is efficiently reduced by vetoing events containing a $b$-tagged jet with $p_{\mathrm{T}}>20 \mathrm{GeV}$ (see Section 4.2.6).

In order to reduce the dijet background, which has a large uncertainty, two singlelepton transverse mass variables are constructed

$$
m_{\mathrm{T}, \ell_{i}}=\sqrt{2 p_{\mathrm{T}, \ell_{i}} p_{\mathrm{T}}^{\mathrm{miss}, \mathrm{corr}}\left[1-\cos \Delta \phi\left(\ell_{i}, p_{\mathrm{T}}^{\mathrm{miss}, \mathrm{corr}}\right)\right]} .
$$

In processes where $\boldsymbol{p}_{\mathrm{T}}^{\text {miss,corr }}$ is collinear with a lepton, such as those with a leptonic hadron or $\tau$ decay, $\max \left(m_{\mathrm{T}, \ell}\right)$ tends to peak at lower values (see Figure 4.16). Therefore we require $\max \left(m_{\mathrm{T}, \ell}\right)>50 \mathrm{GeV}$ in the $e \mu / \mu e$ channels. In the $e e / \mu \mu$ channels, the tighter $p_{\mathrm{T}}^{\text {inv }}$ cuts reject the dijet background so a $\max \left(m_{\mathrm{T}, \ell}\right)$ cut is not needed.

At this stage, the $Z / \gamma^{*} \rightarrow \tau \tau$ background dominates the $e \mu / \mu e$ channels. To aid discrimination, a ditau mass is constructed:

$$
m_{\tau \tau}= \begin{cases}m_{\ell \ell} / \sqrt{x_{1} x_{2}} & \text { if } x_{1}, x_{2}>0 \\ 0 & \text { otherwise }\end{cases}
$$

where $x_{i}$ is the $p_{\mathrm{T}}$ fraction of the $i$ th $\tau$ imparted to the $i$ th lepton. As $x_{i}$ cannot be directly measured using individual neutrino momenta, $p_{\mathrm{T}}^{\text {miss,corr }}$ is split by assuming the $\tau$ decays collinearly, i.e. $\Delta \phi\left(\ell, \nu_{\tau} \nu_{\ell}\right)=0$ [121]. This approximation is reasonable since each $\tau$ has large $p_{\mathrm{T}}$. We require $m_{\tau \tau}<m_{Z}-25 \mathrm{GeV}$ (see Figure 4.16). In rare cases where the ditau system is poorly reconstructed (i.e. $\left.x_{i}<0\right)$, the event is accepted.

After the signal topology selection, the $Z / \gamma^{*} \rightarrow \ell \ell$ background is suppressed in the $e e / \mu \mu$ channels by requiring $p_{\mathrm{T}, \mathrm{rel}}^{\text {miss }}>35 \mathrm{GeV}$ and $f_{\text {recoil }}<0.1$ (see Figure 4.17 ). In the 1-jet bin, the $f_{\text {recoil }}$ definition is altered with respect to (4.8): the quadrant $\wedge$ is centred upon $-\boldsymbol{p}_{\mathrm{T}, \ell \ell j}$ and the denominator becomes $p_{\mathrm{T}, \ell \ell j}$. This is because a hard jet has been found, and so it is the dilepton + jet system that must be balanced by the soft recoil.

The $m_{\mathrm{T}}$ distributions of the selected 1-jet events are shown in Figure 4.18. 

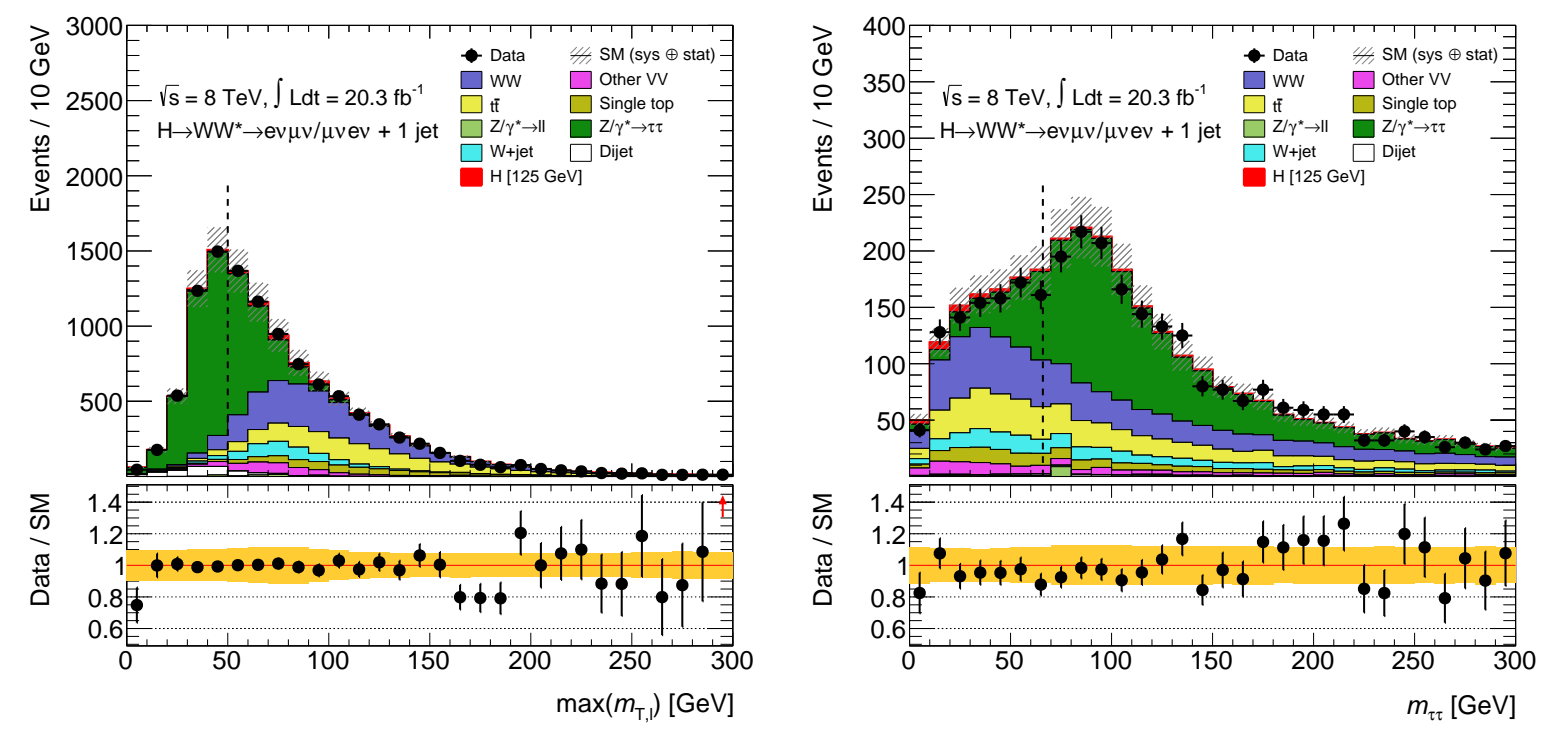

Figure 4.16: The $\max \left(m_{\mathrm{T}, \ell}\right)$ (left) and $m_{\tau \tau}$ (right) distributions in the $e \mu / \mu e$ channels. The selection removes events to the left of the dashed line in the $\max \left(m_{\mathrm{T}, \ell}\right)$ plot, and removes events to the right of the dashed line in the $m_{\tau \tau}$ plot.
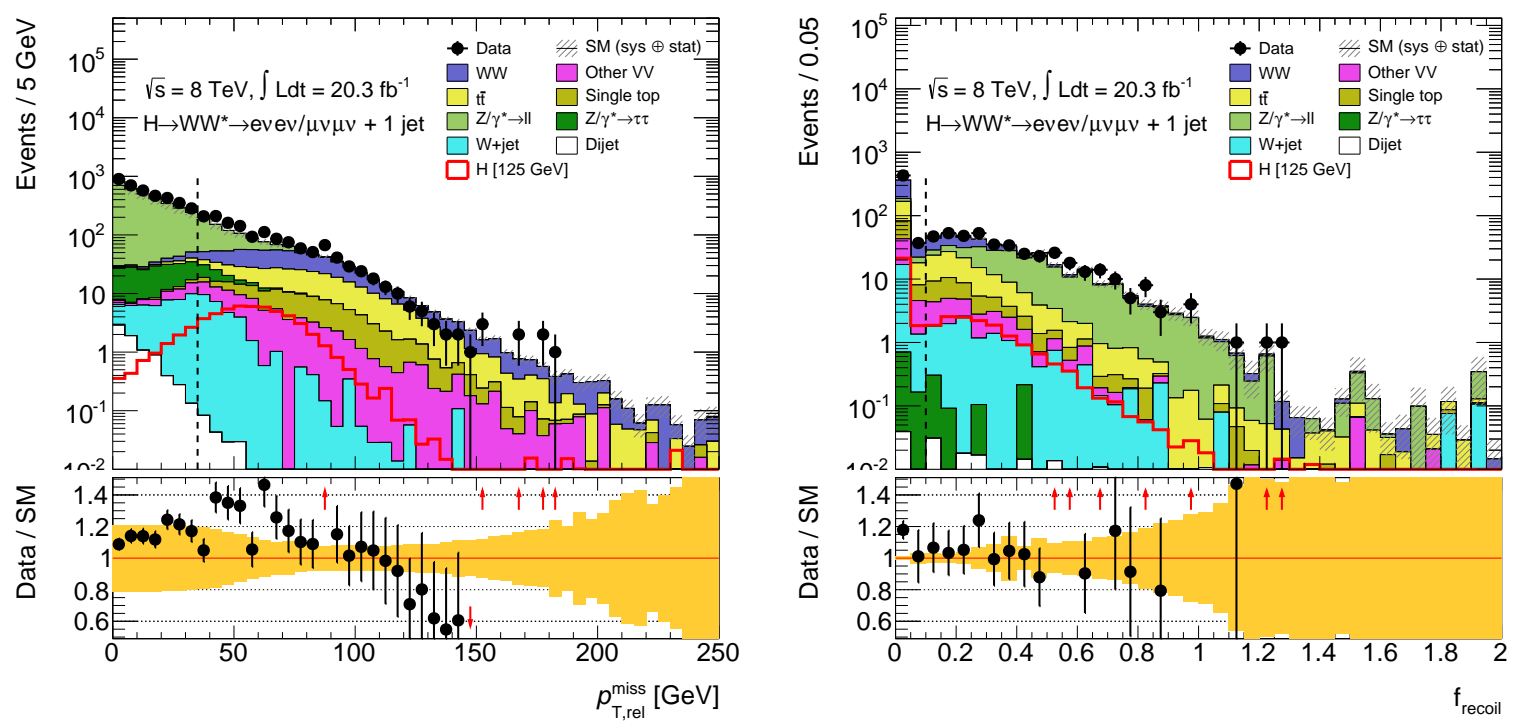

Figure 4.17: The $p_{\mathrm{T}, \mathrm{mel}}^{\text {miss }}$ (left) and $f_{\text {recoil }}$ (right) distributions in the $e e / \mu \mu$ channels. The subsequent selection removes events to the left of the dashed line in the $p_{\mathrm{T}, \mathrm{rel}}^{\text {miss }}$ plot, and removes events to the right of the dashed line in the $f_{\text {recoil }}$ plot. 

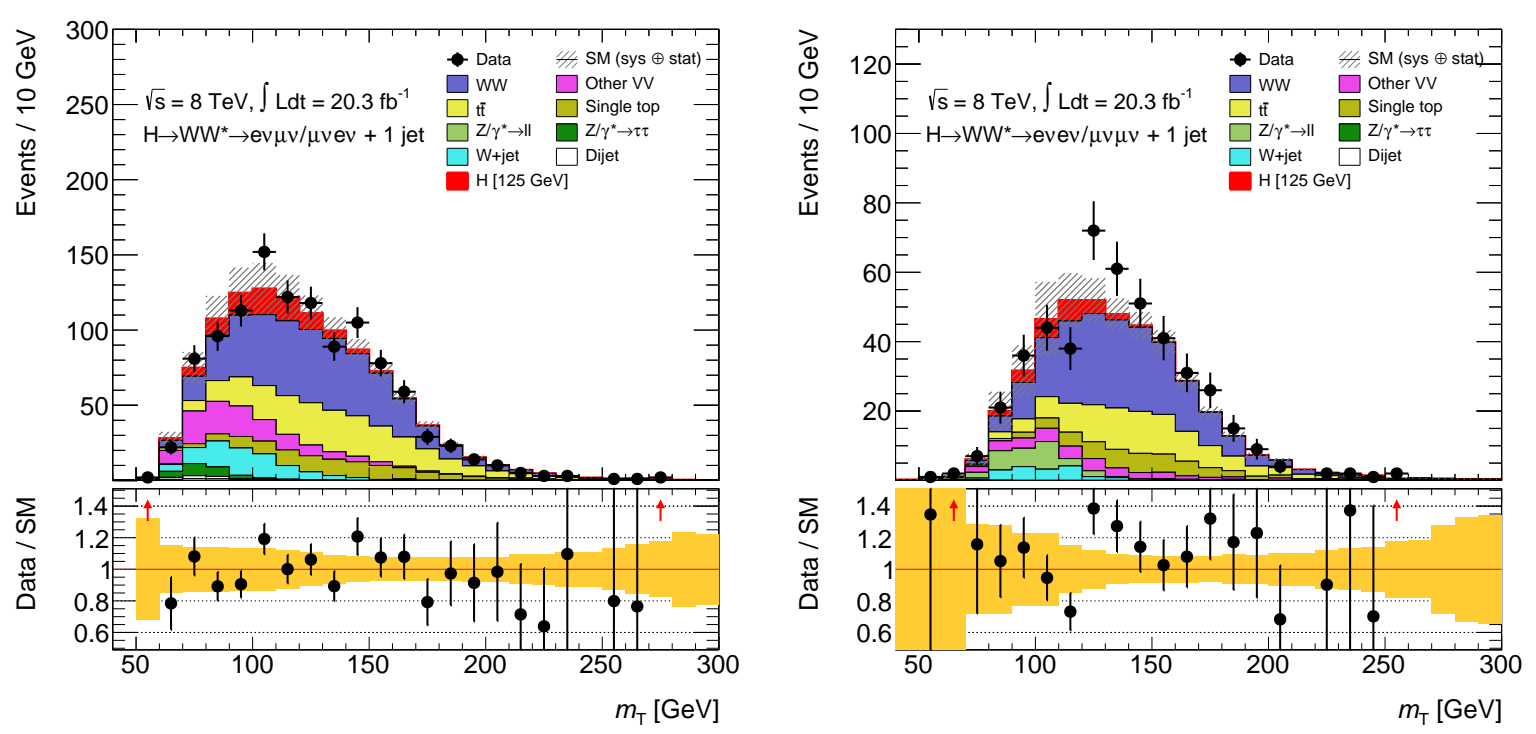

Figure 4.18: The $m_{\mathrm{T}}$ distribution of the selected 1-jet events, in the $e \mu / \mu e$ (left) and $e e / \mu \mu$ (right) channels.

\subsection{7 $\geq 2$-jet selection}

Only the $e \mu / \mu e$ channels are used in the $\geq 2$-jet bin, which is dominated by top background (see Figure 4.12). In the following, observable definitions using only two jets refer to the two hardest jets. As in the 1-jet bin, the top background is greatly suppressed by vetoing events featuring a $b$-tagged jet with $p_{\mathrm{T}}>20 \mathrm{GeV}$ (see Figure 4.19). Also, the $Z / \gamma^{*} \rightarrow \tau \tau$ background is again suppressed by the requirement $m_{\tau \tau}<m_{Z}-25 \mathrm{GeV}$ (see Figure 4.19).

At this point, it is necessary to contextualise the analysis. Although this thesis describes the search for the ggF production mode, searches for the other production modes (see Section 1.3) are also performed by the ATLAS collaboration. To simplify the statistical combination of these searches, it is helpful to avoid overlap between their signal regions. This is particularly important for the $\geq 2$-jet bin.

The VBF production mode features two outgoing quarks at LO (see Figure 1.3b). Therefore the $\geq 2$-jet bin is the starting point for the VBF selection. However, the absence of colour exchange between the quarks leads to dramatically different event topologies compared to ggF events in the $\geq 2$-jet bin; VBF events feature two high- $p_{\mathrm{T}}$ jets, separated by a large rapidity gap devoid of hadronic activity. Thus, events containing a softer jet with $p_{\mathrm{T}}>20 \mathrm{GeV}$ within this rapidity gap are removed from the VBF selection. 

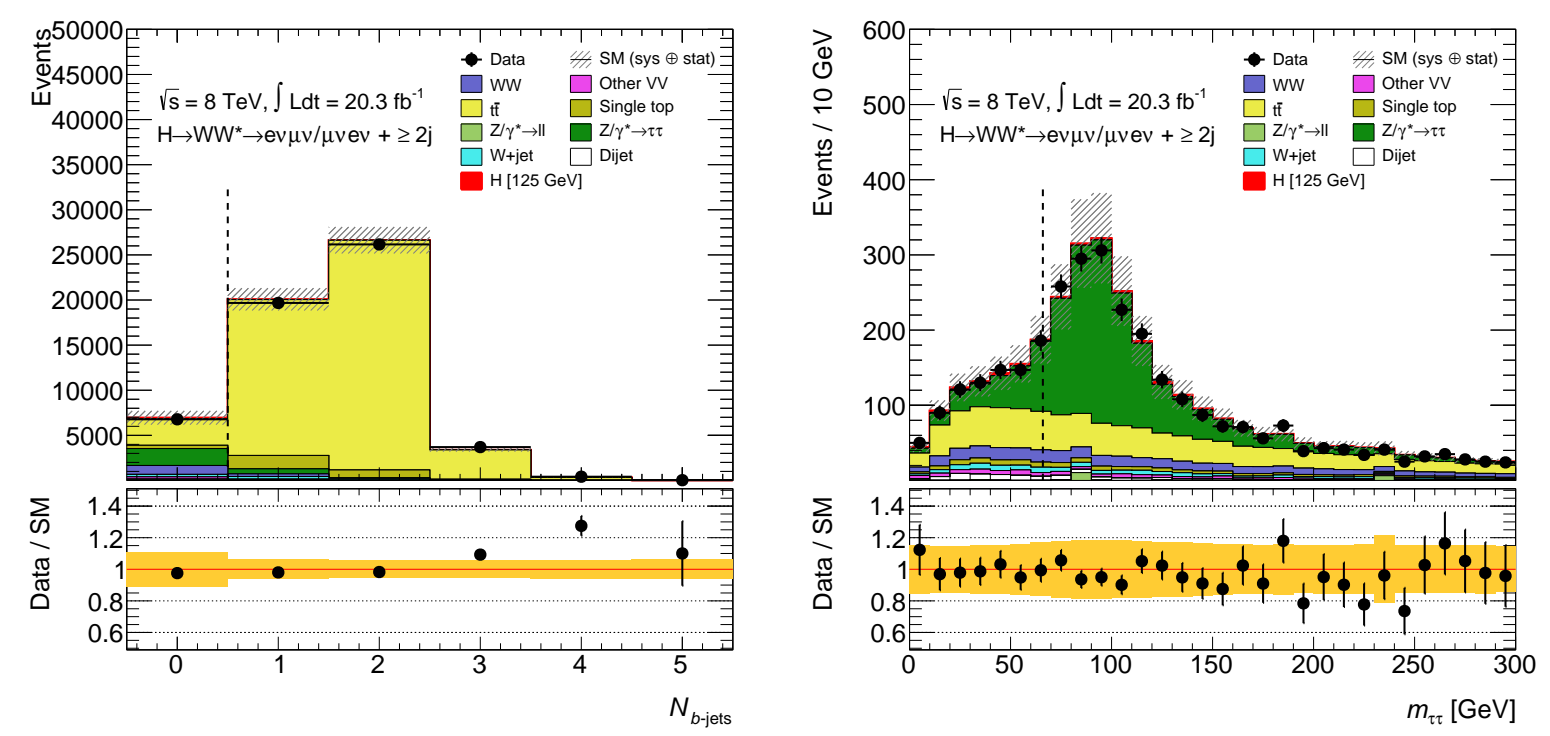

Figure 4.19: The $N_{b \text {-jets }}$ (left) and $m_{\tau \tau}$ (right) distributions in the $e \mu / \mu e$ channels. The subsequent selection removes events to the right of each dashed line.

This is the central jet veto (CJV). The VBF selection also requires that the leptons lie within this rapidity gap, since the Higgs boson is generally produced centrally. This is the outside lepton veto (OLV).

The VBF analysis is then split into two independent analyses: a cut-based analysis and a multivariate analysis. The cut-based VBF analysis requires a high dijet mass, $m_{j j}>600 \mathrm{GeV}$, and a large rapidity gap, $\Delta y(j, j)>3.6$. The multivariate analysis trains a boosted decision tree (BDT) [122] to discriminate VBF events based upon eight input variables:

- VBF topology: $\quad m_{j j}, \quad \Delta y(j, j), \quad \eta_{\ell}$ centrality, $\sum_{\ell, j} m_{\ell j}$

- $H \rightarrow W W$ decay: $m_{\ell \ell}, \quad \Delta \phi(\ell, \ell), m_{\mathrm{T}}$

- top suppression: $\quad p_{\mathrm{T}, \text { tot }}$

$\eta_{\ell}$ centrality characterises how close the leptons are to the jets. $\sum_{\ell, j} m_{\ell j}$ sums the masses of the four lepton + jet systems possible with the two hardest jets; this quantity is higher in VBF events due to the large separations involved. $p_{\mathrm{T}, \text { tot }}$ is the magnitude of the sum of $\boldsymbol{p}_{\mathrm{T}}^{\text {miss,corr }}$ and the $\boldsymbol{p}_{\mathrm{T}}$ of each lepton and jet, capturing the soft hadronic recoil, which is larger in top background events. The BDT scores events between $100 \%$ signal-like $(+1)$ and $100 \%$ background-like $(-1)$, where ggF is considered a background. Events with a score greater than -0.48 are selected for the VBF analysis. 


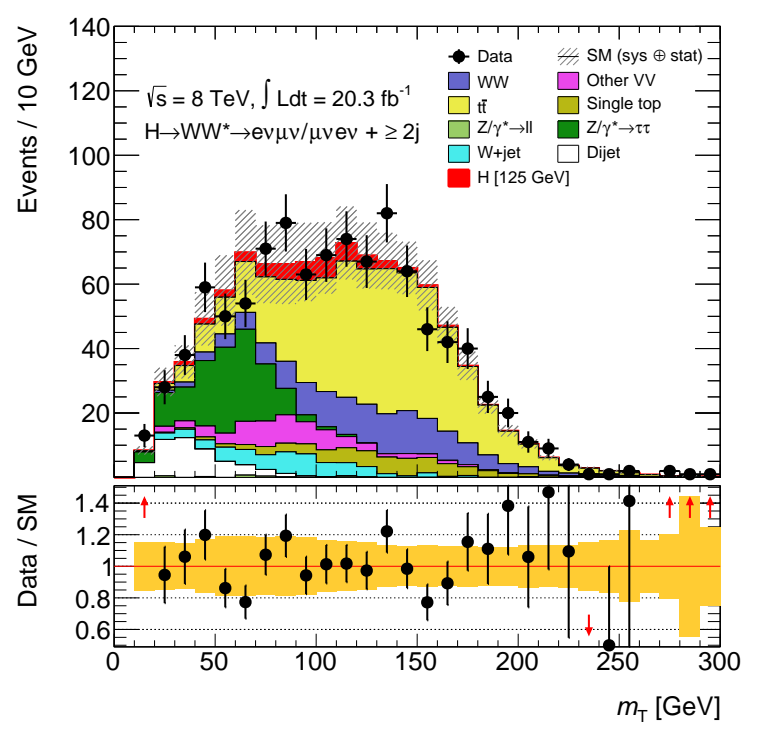

Figure 4.20: The $m_{\mathrm{T}}$ distribution of the selected $\geq 2$-jet events, in the $e \mu / \mu e$ channels.

Another search considers the $W H$ and $Z H$ production modes (collectively known as $V H$ ), where the vector boson decays hadronically (see Figure 1.3c). Again, the $\geq 2$-jet bin is the starting point for this selection. As with VBF, the jet kinematics can be used to distinguish this from ggF. First, a small rapidity gap is required, $\Delta y(j, j)<1.2$. Second, the dijet system must have a mass corresponding to a $W$ or $Z$ boson, $\left|m_{j j}-85\right|<15 \mathrm{GeV}$.

To maintain orthogonality with both the VBF analyses and the $V H$ analysis, the ggF analysis requires that events in the $\geq 2$-jet bin must fail at least one cut from each selection. That is, an event must fail either the CJV, the OLV or the BDT score VBF cut and it must fail either the CJV, the OLV, the $m_{j j}$ or the $\Delta y(j, j) \mathrm{VBF}$ cut and it must fail either the $\Delta y(j, j)$ or the $m_{j j} V H$ cut.

Finally, the usual signal topology selection is made, $m_{\ell \ell}<55 \mathrm{GeV}$ and $\Delta \phi(\ell, \ell)<1.8$. The $m_{\mathrm{T}}$ distribution of the selected $\geq 2$-jet events is shown in Figure 4.20. 


\subsubsection{Summary of signal regions}

The entire event selection is concisely summarised in Table 4.5. The ee and $\mu \mu$ channels are combined into a single "same-flavour" channel, $e e+\mu \mu$. In total, there are 8 different signal regions: $\{e \mu, \mu e, e e+\mu \mu\} \otimes\{0$-jet,1-jet $\} \oplus\{e \mu, \mu e\} \otimes\{\geq 2$-jet $\}$.

Chapter 8 shall describe how statistical limits are extracted from these signal regions, by comparing the observed $m_{\mathrm{T}}$ distributions to those expected. However, some key points are summarised here. The majority of the sensitivity lies in the 0 -jet and 1-jet bins of the $e \mu / \mu e$ channels. In fact, the sensitivity is further optimised by splitting each of these four signal regions into three bins of $p_{\mathrm{T}, \ell}^{\text {sublead }}$ and two bins of $m_{\ell \ell}$ (see Figure 4.21). This equates to performing a three-dimensional fit of $m_{\mathrm{T}}, m_{\ell \ell}$ and $p_{\mathrm{T}, \ell}^{\text {sublead }}$. In the other signal regions, a simple one-dimensional $m_{\mathrm{T}}$ fit is used. 


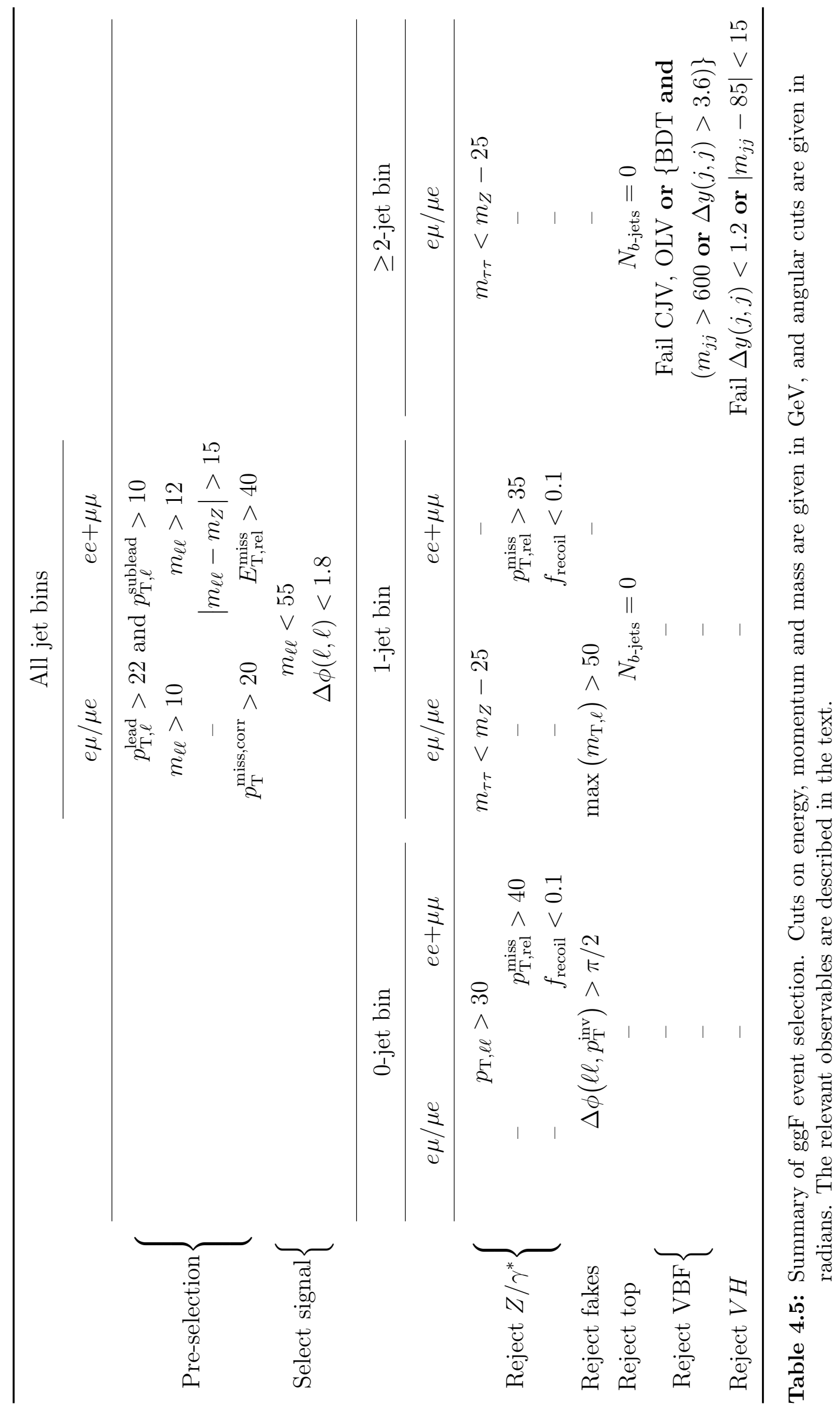



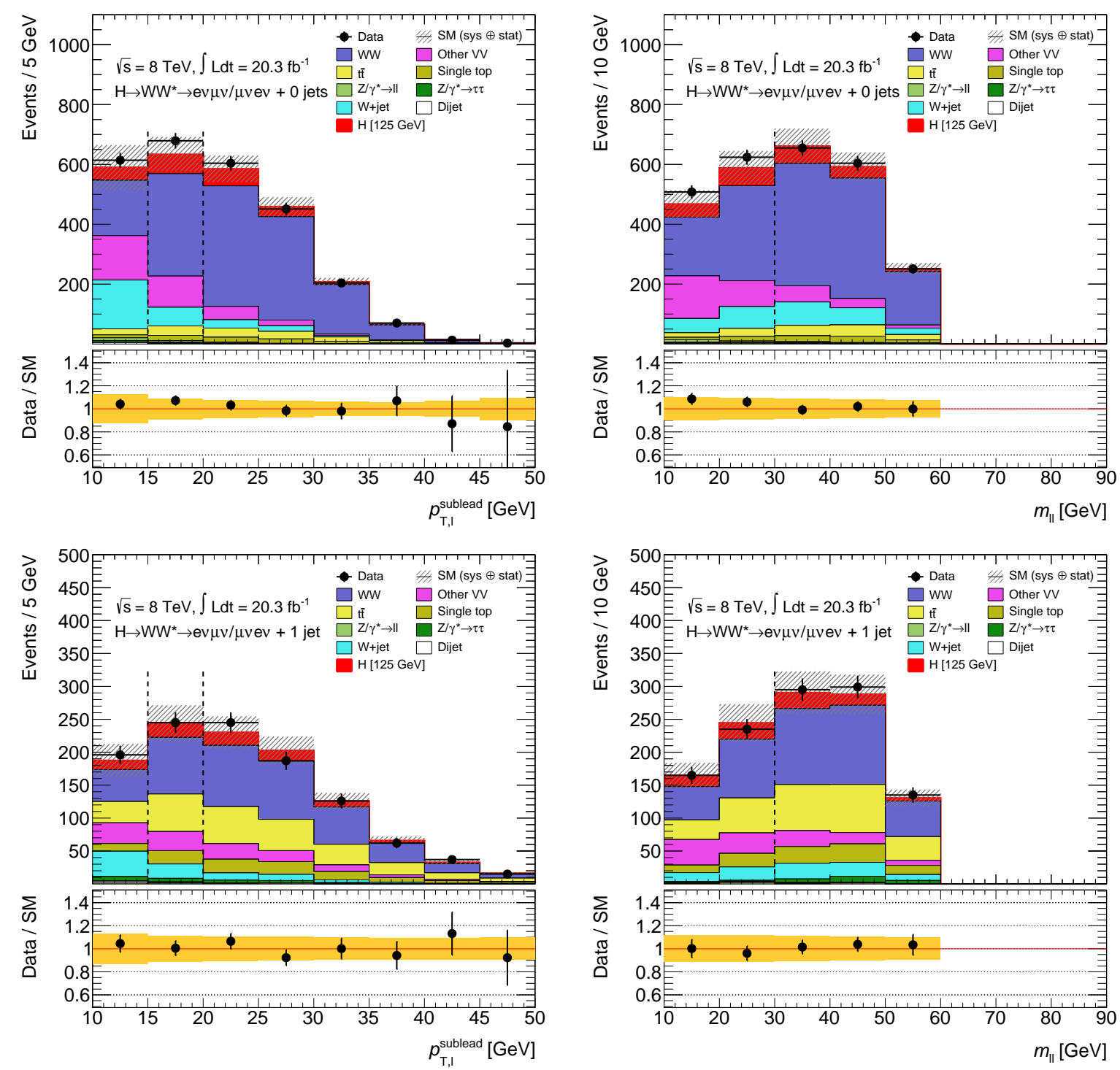

Figure 4.21: The $p_{\mathrm{T}, \ell}^{\text {sublead }}$ (left) and $m_{\ell \ell}$ (right) distributions in the 0-jet (top) and 1-jet (bottom) signal regions of the $e \mu / \mu e$ channels. The dashed lines indicate how the signal regions are split in the fit. 


\section{Chapter 5}

\section{Signal modelling}

This thesis describes a search for the ggF production mode (see Figure 5.1). This exhibits large theoretical uncertainties due to higher order corrections, ${ }^{1}$ and so its cross section is calculated at NNLO+NNLL in QCD and NLO in EW (see Figure 1.4). PDF uncertainties are also significant, since the low- $x$ gluon is relatively poorly constrained (see Figure 2.2). Calculations are also sensitive to the treatment of quark masses in the loop.

Section 5.1 considers the significant theoretical issues introduced by the jet binning of the analysis, and then the MC modelling of ggF is discussed in Section 5.2.

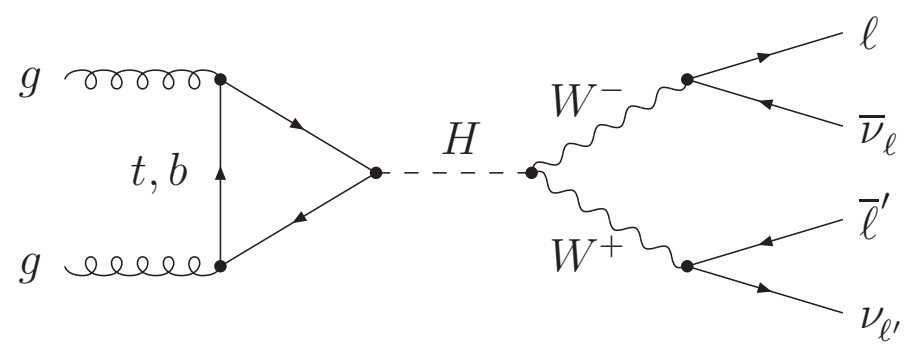

Figure 5.1: Leading order Feynman diagram for gluon-gluon fusion (ggF).

\footnotetext{
${ }^{1}$ The poor convergence of the ggF perturbative series, with respect to similar $q \bar{q}$-initiated processes, is thought to be due to the larger colour factor of the gluon.
} 


\subsection{Jet-binned cross sections}

The $g g \rightarrow H \rightarrow W W$ analysis is binned according to jet multiplicity, in order to exploit the vastly different background compositions in each jet bin (see Figure 4.12); the 0jet, 1 -jet and $\geq 2$-jet bins each have dedicated event selection criteria. Uncertainties in the expected ggF cross section must be evaluated separately for each jet bin, and correlations between these bins must be considered when they are combined. Perturbative uncertainties in the jet binning itself are considered independently from the other selection criteria, since they possess additional subtleties described below.

\subsubsection{Perturbative uncertainties in jet-binned cross sections}

Consider splitting a cross section into two parts: an exclusive 0 -jet cross section, $\sigma_{0}$, and an inclusive $\geq 1$-jet cross section, $\sigma_{\geq 1}$ :

$$
\sigma_{\text {tot }}=\sigma_{0}\left(p_{\mathrm{T}}^{\text {cut }}\right)+\sigma_{\geq 1}\left(p_{\mathrm{T}}^{\text {cut }}\right)
$$

where $p_{\mathrm{T}}^{\text {cut }}$ is the jet $p_{\mathrm{T}}$ threshold [43]. In $\sigma_{\geq 1}$, the requirement of a jet with $p_{\mathrm{T}}>p_{\mathrm{T}}^{\text {cut }}$ introduces double logarithmic contributions $\alpha_{\mathrm{S}}^{k+m} L^{2 m}$, where $L \sim \ln \left(p_{\mathrm{T}}^{\text {cut }} / Q\right)$ and $Q$ is the scale of the hard scatter $\left(Q=m_{H}\right.$ is typical for $\left.\mathrm{ggF}\right)$. These terms are analogous to the logarithms introduced by soft gluon emission (see Section 2.1.3), though they depend upon the process and also the jet algorithm and parameters $\left(e . g\right.$. anti- $k_{\mathrm{T}}$ with $\left.R=0.4\right)$.

The schematic structures of the two inclusive cross sections are

$$
\begin{array}{llll}
\sigma_{\text {tot }} \sim \alpha_{\mathrm{S}}^{k}\{1 & +\alpha_{\mathrm{S}} & +\alpha_{\mathrm{S}}^{2} & \left.+\mathcal{O}\left(\alpha_{\mathrm{S}}^{3}\right)\right\} \\
\sigma_{\geq 1} \sim \alpha_{\mathrm{S}}^{k}\{ & \alpha_{\mathrm{S}}\left(L^{2}+L+1\right) & +\alpha_{\mathrm{S}}^{2}\left(L^{4}+L^{3}+L^{2}+L+1\right) & \left.+\mathcal{O}\left(\alpha_{\mathrm{S}}^{3} L^{6}\right)\right\} .
\end{array}
$$

When $p_{\mathrm{T}}^{\text {cut }} \ll m_{H}$, the logarithms can overcome the $\alpha_{\mathrm{S}}$ suppression and provide significant corrections to $\sigma_{\geq 1}$. When these corrections are similar in size to the perturbative corrections to $\sigma_{\text {tot }}$, the scale dependence of $\sigma_{0}=\sigma_{\text {tot }}-\sigma_{\geq 1}$ is reduced by cancellations between the two series. This suggests that naïvely varying $\mu_{\mathrm{R}}$ and $\mu_{\mathrm{F}}$ might underestimate perturbative uncertainties. This is confirmed in Figure 5.2, which shows that the cancellations at $p_{\mathrm{T}}^{\text {cut }}=25 \mathrm{GeV}$ (used in the $H \rightarrow W W$ analysis) are rather extreme.

When discussing uncertainties in jet-binned cross sections, it is convenient to consider a general parametrisation of the covariance matrix [123]. In the $\left\{\sigma_{0}, \sigma_{\geq 1}\right\}$ basis, the 
covariance matrix is decomposed into two uncertainty sources

$$
\begin{aligned}
C & =C^{\text {yield }}+C^{\text {migration }} \\
& =\left(\begin{array}{cc}
\left(\Delta_{0}^{\mathrm{y}}\right)^{2} & \Delta_{0}^{\mathrm{y}} \Delta_{\geq 1}^{\mathrm{y}} \\
\Delta_{0}^{\mathrm{y}} \Delta_{\geq 1}^{\mathrm{y}} & \left(\Delta_{\geq 1}^{\mathrm{y}}\right)^{2}
\end{array}\right)+\left(\Delta_{0 \rightarrow}^{\mathrm{mig}}\right)^{2}\left(\begin{array}{ll}
+1 & -1 \\
-1 & +1
\end{array}\right)
\end{aligned}
$$

where $\Delta_{N}^{\mathrm{y}}$ is the uncertainty in the $N$-jet cross section due to the yield uncertainty, and $\Delta_{0 \rightarrow}^{\mathrm{mig}}$ is the cross section uncertainty due to bin migrations. The yield component is fully correlated between jet bins, though can affect each with different magnitudes. The migration component is fully anti-correlated and affects both bins equally, conserving the normalisation. In the statistical model (see Section 8.2.2), these two sources are treated as nuisance parameters $\theta$ with uncertainty amplitudes $\nu(\Delta \theta)$ in the $\left\{\sigma_{0}, \sigma_{\geq 1}\right\}$ basis:

$$
\begin{aligned}
\theta^{\text {yield }}: & \left(\begin{array}{rr}
\Delta_{0}^{\mathrm{y}}, & \Delta_{\geq 1}^{\mathrm{y}}
\end{array}\right) \\
\theta_{0 \rightarrow}^{\mathrm{mig}}: & \left(\begin{array}{cc}
\Delta_{0 \rightarrow}^{\mathrm{mig}}, & -\Delta_{0 \rightarrow}^{\mathrm{mig}}
\end{array}\right) .
\end{aligned}
$$

Different prescriptions for evaluating perturbative uncertainties are defined by their choice of $\Delta_{0}^{\mathrm{y}}, \Delta_{\geq 1}^{\mathrm{y}}$ and $\Delta_{0 \rightarrow}^{\mathrm{mig}}$. This includes a choice of which observables to measure uncertainties in, and also the method of measuring the uncertainties. For example, the naïve prescription described above is equivalent to choosing

$$
\text { Naïve: } \quad \Delta_{0}^{\mathrm{y}}=\Delta \sigma_{0}, \quad \Delta_{\geq 1}^{\mathrm{y}}=\Delta \sigma_{\geq 1}, \quad \Delta_{0 \rightarrow}^{\mathrm{mig}}=0
$$

where uncertainties are evaluated at fixed order by varying $\mu_{\mathrm{R}}$ and $\mu_{\mathrm{F}}$.

In the $H \rightarrow W W$ analysis, there is also an exclusive 1 -jet bin. The second jet veto introduces an additional source of migrations, now between the 1 -jet and $\geq 2$-jet bins. 
Therefore, in the $\left\{\sigma_{0}, \sigma_{1}, \sigma_{\geq 2}\right\}$ basis, the covariance matrix has three components

$$
\begin{aligned}
C & =\left(\begin{array}{ccc}
\left(\Delta_{0}^{\mathrm{y}}\right)^{2} & \Delta_{0}^{\mathrm{y}} \Delta_{1}^{\mathrm{y}} & \Delta_{0}^{\mathrm{y}} \Delta_{\geq 2}^{\mathrm{y}} \\
\Delta_{0}^{\mathrm{y}} \Delta_{1}^{\mathrm{y}} & \left(\Delta_{1}^{\mathrm{y}}\right)^{2} & \Delta_{1}^{\mathrm{y}} \Delta_{\geq 2}^{\mathrm{y}} \\
\Delta_{0}^{\mathrm{y}} \Delta_{\geq 2}^{\mathrm{y}} & \Delta_{1}^{\mathrm{y}} \Delta_{\geq 2}^{\mathrm{y}} & \left(\Delta_{\geq 2}^{\mathrm{y}}\right)^{2}
\end{array}\right) \\
& +\left(\Delta_{0 \rightarrow}^{\mathrm{mig}}\right)^{2}\left(\begin{array}{ccc}
+1 & -(1-\rho) & -\rho \\
-(1-\rho) & (1-\rho)^{2} & \rho(1-\rho) \\
-\rho & \rho(1-\rho) & \rho^{2}
\end{array}\right)+\left(\Delta_{1 \rightarrow}^{\mathrm{mig}}\right)^{2}\left(\begin{array}{ccc}
0 & 0 & 0 \\
0 & +1 & -1 \\
0 & -1 & +1
\end{array}\right)
\end{aligned}
$$

where $\rho$ is the fraction of migrations from the 0 -jet bin $\left(\Delta_{0 \rightarrow}^{\mathrm{mig}}\right)$ that enter the $\geq 2$-jet bin. In this case, there are three nuisance parameters $\theta$ with uncertainty amplitudes $\nu(\theta)$ in the $\left\{\sigma_{0}, \sigma_{1}, \sigma_{\geq 2}\right\}$ basis:

$$
\begin{aligned}
& \theta^{\text {yield }}:\left(\begin{array}{ccc}
\Delta_{0}^{\mathrm{y}}, & \Delta_{1}^{\mathrm{y}}, & \Delta_{\geq 2}^{\mathrm{y}}
\end{array}\right) \\
& \theta_{0 \rightarrow}^{\mathrm{mig}}:\left(\Delta_{0 \rightarrow}^{\mathrm{mig}},-(1-\rho) \Delta_{0 \rightarrow}^{\mathrm{mig}},-\rho \Delta_{0 \rightarrow}^{\mathrm{mig}}\right)
\end{aligned}
$$

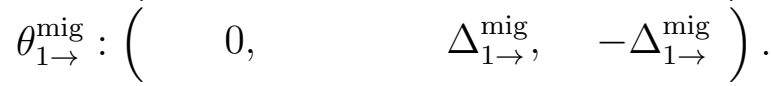

So it is $\Delta_{0}^{\mathrm{y}}, \Delta_{1}^{\mathrm{y}}, \Delta_{\geq 2}^{\mathrm{y}}, \Delta_{0 \rightarrow}^{\mathrm{mig}}, \Delta_{1 \rightarrow}^{\mathrm{mig}}$ and $\rho$ that must be determined. Two different prescriptions for evaluating these shall now be examined.

\subsubsection{Combined inclusive prescription}

The combined inclusive (CI) prescription $^{2}$ [124] uses scale variations of $\sigma_{\geq 1}$ and $\sigma_{\geq 2}$ to probe the size of the higher order logarithmic corrections, and uses these to estimate the bin migration uncertainties. It therefore chooses

$$
\begin{aligned}
& \text { CI: } \quad \Delta_{0}^{\mathrm{y}}=\Delta \sigma_{\text {tot }}, \quad \Delta_{1}^{\mathrm{y}}=0, \quad \Delta_{\geq 2}^{\mathrm{y}}=0 \text {, } \\
& \Delta_{0 \rightarrow}^{\mathrm{mig}}=\Delta \sigma_{\geq 1}, \quad \Delta_{1 \rightarrow}^{\mathrm{mig}}=\Delta \sigma_{\geq 2}, \quad \rho=0
\end{aligned}
$$

where $\Delta \sigma_{\text {tot }}, \Delta \sigma_{\geq 1}$ and $\Delta \sigma_{\geq 2}$ are evaluated, at a common fixed order in $\alpha_{\mathrm{S}}$, through variations of the $\mu_{\mathrm{R}}$ and $\mu_{\mathrm{F}}$ scales.

\footnotetext{
${ }^{2}$ The CI prescription is also called the Stewart-Tackmann prescription, after its original proponents.
} 

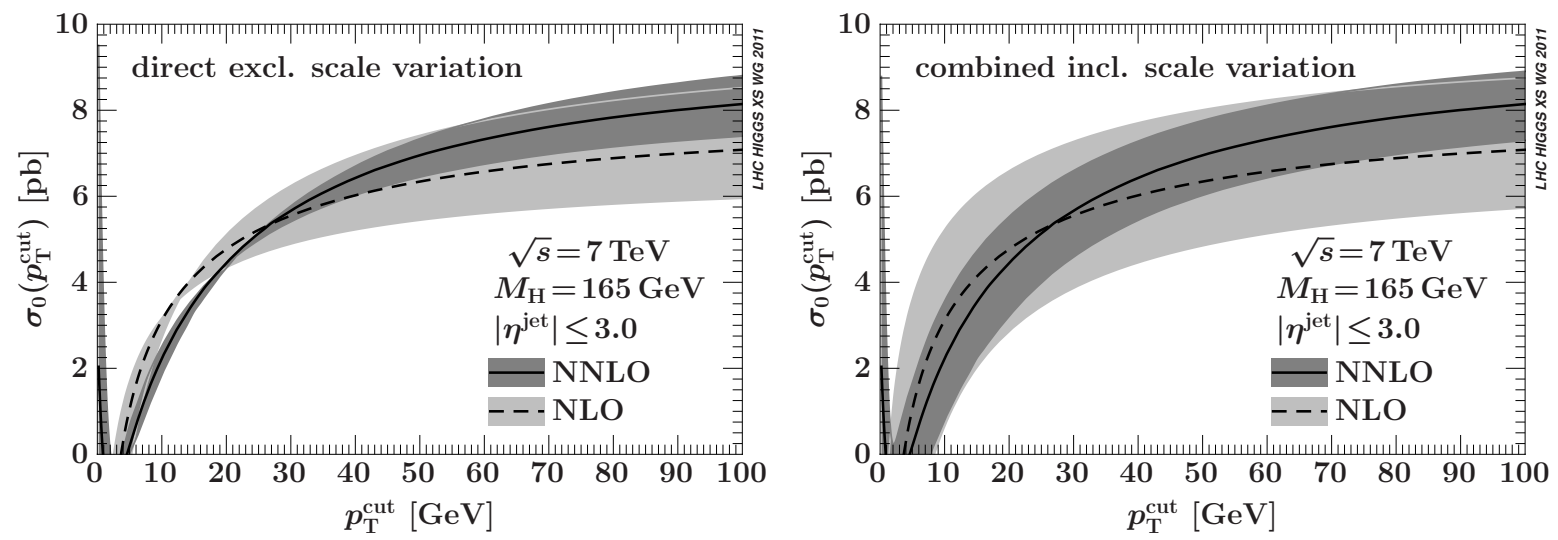

Figure 5.2: The exclusive 0 -jet ggF cross section versus the jet $p_{\mathrm{T}}$ threshold [43]. The bands show the perturbative uncertainties evaluated using the naïve prescription (left) and the combined inclusive prescription (right).

This is equivalent to assuming inclusive cross sections have uncorrelated uncertainties

$$
\text { CI: } \quad \sigma_{N}=\sigma_{\geq N}-\sigma_{\geq N+1} \quad \Rightarrow \quad \Delta \sigma_{N}^{2}=\Delta \sigma_{\geq N}^{2}+\Delta \sigma_{\geq N+1}^{2} .
$$

Although this assumption is not believed to be exact, the CI prescription offers a practical solution to the cancellations described in Section 5.1.1 (see Figure 5.2). It ensures that uncertainties in exclusive cross sections are larger than those in the corresponding inclusive cross section, i.e. $\Delta \sigma_{N} \geq \Delta \sigma_{\geq N}$, with the equality valid in the large- $p_{\mathrm{T}}^{\text {cut }}$ limit.

It should be emphasised that each inclusive cross section must be evaluated at the same order in $\alpha_{\mathrm{S}}\left(e . g . \sigma_{\text {tot }}^{\mathrm{NNO}}, \sigma_{\geq 1}^{\mathrm{NLO}}\right.$ and $\left.\sigma_{\geq 2}^{\mathrm{LO}}\right)$. This can restrict the application of the CI prescription. For example, an exclusive 2-jet bin cannot be added until $\sigma_{\text {tot }}$ is calculated at $\mathrm{N}^{3} \mathrm{LO}$ in QCD. For the ggF contamination to the VBF signal region (defined with a central jet veto), $\sigma_{\geq 2}^{\mathrm{NLO}}$ and $\sigma_{\geq 3}^{\mathrm{LO}}$ can be used since they are evaluated in a significantly different phase space $\left(\Delta \sigma_{\geq 2}^{\mathrm{NLO}}\right.$ and $\Delta \sigma_{\geq 2}^{\mathrm{LO}}$ are treated as fully correlated).

HNNLO [125] is used to compute $\sigma_{\text {tot }}^{\mathrm{NNO}}, \sigma_{\geq 1}^{\mathrm{NLO}}$ and $\sigma_{\geq 2}^{\mathrm{LO}}$. However, the CI prescription can be improved by using the NNLO+NNLL $(\mathrm{QCD})+\mathrm{NLO}(\mathrm{EW}) \sigma_{\text {tot }}$ calculation [10], which has smaller perturbative uncertainties than $\sigma_{\text {tot }}^{\mathrm{NNLO}}$. In order to combine these results whilst preserving the total normalisation, the jet bin fractions $f_{N}=\sigma_{N} / \sigma_{\text {tot }}$ from 


\begin{tabular}{rccr}
\hline \multicolumn{1}{c}{$i$} & $f_{i}=\sigma_{i} / \sigma_{\text {tot }}$ & $\sigma_{i}(\mathrm{pb})$ & $\Delta \sigma_{i} / \sigma_{i}$ \\
\hline$\geq 0$ & - & 19.27 & $7.8 \%$ \\
$\geq 1$ & - & - & $20.2 \%$ \\
$\geq 2$ & - & - & $69.7 \%$ \\
\hline 0 & 0.614 & 11.83 & $18.0 \%$ \\
1 & 0.267 & 5.15 & $42.6 \%$ \\
$\geq 2$ & - & 2.29 & $69.7 \%$ \\
\hline
\end{tabular}

Table 5.1: Inputs and outputs (boxed) of the combined inclusive prescription for ggF, with $m_{H}=125 \mathrm{GeV}$ and $\sqrt{s}=8 \mathrm{TeV}$. All inputs are computed by HNNLO except $\sigma_{\text {tot }}$ and $\Delta \sigma_{\text {tot }}$, which are from reference [10].

HNNLO are used to propagate the $\Delta \sigma_{\geq N}$ to $\Delta \sigma_{N}$ :

$$
\begin{aligned}
& \delta \sigma_{0}^{2}=\frac{1}{f_{0}^{2}} \delta \sigma_{\text {tot }}^{2}+\left(\frac{1}{f_{0}}-1\right)^{2} \delta \sigma_{\geq 1}^{2} \\
& \delta \sigma_{1}^{2}=\left(\frac{1-f_{0}}{f_{1}}\right)^{2} \delta \sigma_{\geq 1}^{2}+\left(\frac{1-f_{0}}{f_{1}}-1\right)^{2} \delta \sigma_{\geq 2}^{2}
\end{aligned}
$$

where $\delta \sigma_{i}=\Delta \sigma_{i} / \sigma_{i}$. This assumes the uncertainties are Gaussian distributed, though the nuisance parameters are constrained by log-normal distributions in the statistical model (see Section 8.2.2). The $\Delta \sigma_{\geq N}$ are evaluated via independent variation of $\mu_{\mathrm{R}}$ and $\mu_{\mathrm{F}}$ in the range $m_{H} / 4 \leq \mu_{\mathrm{R}}, \mu_{\mathrm{F}} \leq m_{H}$, whilst observing the constraint $1 / 2 \leq \mu_{\mathrm{R}} / \mu_{\mathrm{F}} \leq 2$. These are then propagated to $\Delta \sigma_{N}$ using (5.12) and (5.13), as shown in Table 5.1.

\subsubsection{Jet veto efficiency prescription}

The jet veto efficiency (JVE) prescription [126,127] considers each exclusive cross section as a product of the total cross section and jet veto efficiencies

$$
\begin{aligned}
\sigma_{0} & =\sigma_{\text {tot }} \epsilon_{0} \\
\sigma_{1} & =\sigma_{\text {tot }}\left(1-\epsilon_{0}\right) \epsilon_{1} \\
\sigma_{\geq 2} & =\sigma_{\text {tot }}\left(1-\epsilon_{0}\right)\left(1-\epsilon_{1}\right)
\end{aligned}
$$


where $\epsilon_{0}$ and $\epsilon_{1}$ are the first and second jet veto efficiencies. That is, $\epsilon_{0}=\epsilon_{0}\left(p_{\mathrm{T}}^{\text {cut }}\right)$ is the efficiency of a veto upon jets with $p_{\mathrm{T}}>p_{\mathrm{T}}^{\text {cut }}$, and $\epsilon_{1}=\epsilon_{1}\left(p_{\mathrm{T}}^{\text {sel }}, p_{\mathrm{T}}^{\text {cut }}\right)$ is the efficiency of a veto upon additional jets with $p_{\mathrm{T}}>p_{\mathrm{T}}^{\text {cut }}$ given that there is already a jet with $p_{\mathrm{T}}>p_{\mathrm{T}}^{\text {sel }}$. In the $H \rightarrow W W$ analysis, $p_{\mathrm{T}}^{\text {cut }}=p_{\mathrm{T}}^{\text {sel }}=25 \mathrm{GeV} .^{3}$

The JVE prescription assumes that uncertainties in $\sigma_{\text {tot }}$ and the $\epsilon_{N}$ are uncorrelated, which ensures that uncertainties in exclusive cross sections are larger than those in the total cross section, i.e. $\Delta \sigma_{N}>\Delta \sigma_{\text {tot. }}$. This is equivalent to choosing

$$
\begin{aligned}
& \text { JVE: } \quad \Delta_{0}^{\mathrm{y}}=\Delta \sigma_{\text {tot }} \epsilon_{0}, \quad \Delta_{1}^{\mathrm{y}}=\Delta \sigma_{\text {tot }}\left(1-\epsilon_{0}\right) \epsilon_{1}, \quad \Delta_{\geq 2}^{\mathrm{y}}=\Delta \sigma_{\text {tot }}\left(1-\epsilon_{0}\right)\left(1-\epsilon_{1}\right), \\
& \Delta_{0 \rightarrow}^{\mathrm{mig}}=\Delta \epsilon_{0} \sigma_{\mathrm{tot}}, \quad \Delta_{1 \rightarrow}^{\mathrm{mig}}=\Delta \epsilon_{1} \sigma_{\mathrm{tot}}\left(1-\epsilon_{0}\right), \quad \rho=1-\epsilon_{1} .
\end{aligned}
$$

Thus, the JVE prescription requires six inputs: $\sigma_{\text {tot }}, \epsilon_{0}, \epsilon_{1}, \Delta \sigma_{\text {tot }}, \Delta \epsilon_{0}$ and $\Delta \epsilon_{1}$. As in the CI prescription, $\sigma_{\text {tot }}$ and $\Delta \sigma_{\text {tot }}$ are taken from the NNLO+NNLL(QCD)+NLO(EW) calculation. However, the $\epsilon_{N}$ contain similar cancellations to those discussed in Section 5.1.1, and so the $\Delta \epsilon_{N}$ must be treated with care.

There is an ambiguity in the definition of $\epsilon_{N}$ that is not present in the fixed order $\sigma_{\geq N}$ calculations [126]. For example, considering NNLO terms with respect to the $N$-jet process, three alternative definitions of $\epsilon_{N}$ may be identified

$$
\begin{aligned}
\epsilon_{N}^{(\mathrm{a})} & =1-\frac{\sigma_{\geq N+1}^{\mathrm{NLO}}}{\sigma_{\geq N}^{\mathrm{NNLO}}} \\
\epsilon_{N}^{(\mathrm{b})} & =1-\frac{\sigma_{\geq N+1}^{\mathrm{NLO}}}{\sigma_{\geq N}^{\mathrm{NLO}}} \\
\epsilon_{N}^{(\mathrm{c})} & =1-\frac{\sigma_{\geq N+1}^{\mathrm{NLO}}}{\sigma_{\geq N}^{\mathrm{LO}}}+\left(\frac{\sigma_{\geq N}^{\mathrm{NLO}}}{\sigma_{\geq N}^{\mathrm{LO}}}-1\right) \frac{\sigma_{\geq N+1}^{\mathrm{LO}}}{\sigma_{\geq N}^{\mathrm{LO}}} .
\end{aligned}
$$

Although scheme (a) is the most intuitive definition, schemes (b) and (c) differ by $\mathrm{N}^{3} \mathrm{LO}$ terms and therefore probe higher order corrections. ${ }^{4}$ However, this probing is less susceptible to accidental cancellations than scale variations. Thus, $\epsilon_{N}^{(a)}$ is used as the nominal $\epsilon_{N}$, whilst $\Delta \epsilon_{N}$ is evaluated by scale variations in $\epsilon_{N}^{(a)}$ or by the difference between the schemes, whichever is larger.

\footnotetext{
${ }^{3}$ Since the jets are predominantly central, the raising of $p_{\mathrm{T}}^{\text {cut }}$ to $30 \mathrm{GeV}$ in the forward region is neglected. This approximation is conservative, since the $\Delta \sigma_{N}$ decrease with higher $p_{\mathrm{T}}^{\text {cut }}$.

${ }^{4}$ Processes whose perturbative series converge more quickly, such as $q \bar{q} \rightarrow Z$, exhibit better agreement between schemes. This supports using these schemes to evaluate perturbative uncertainties.
} 

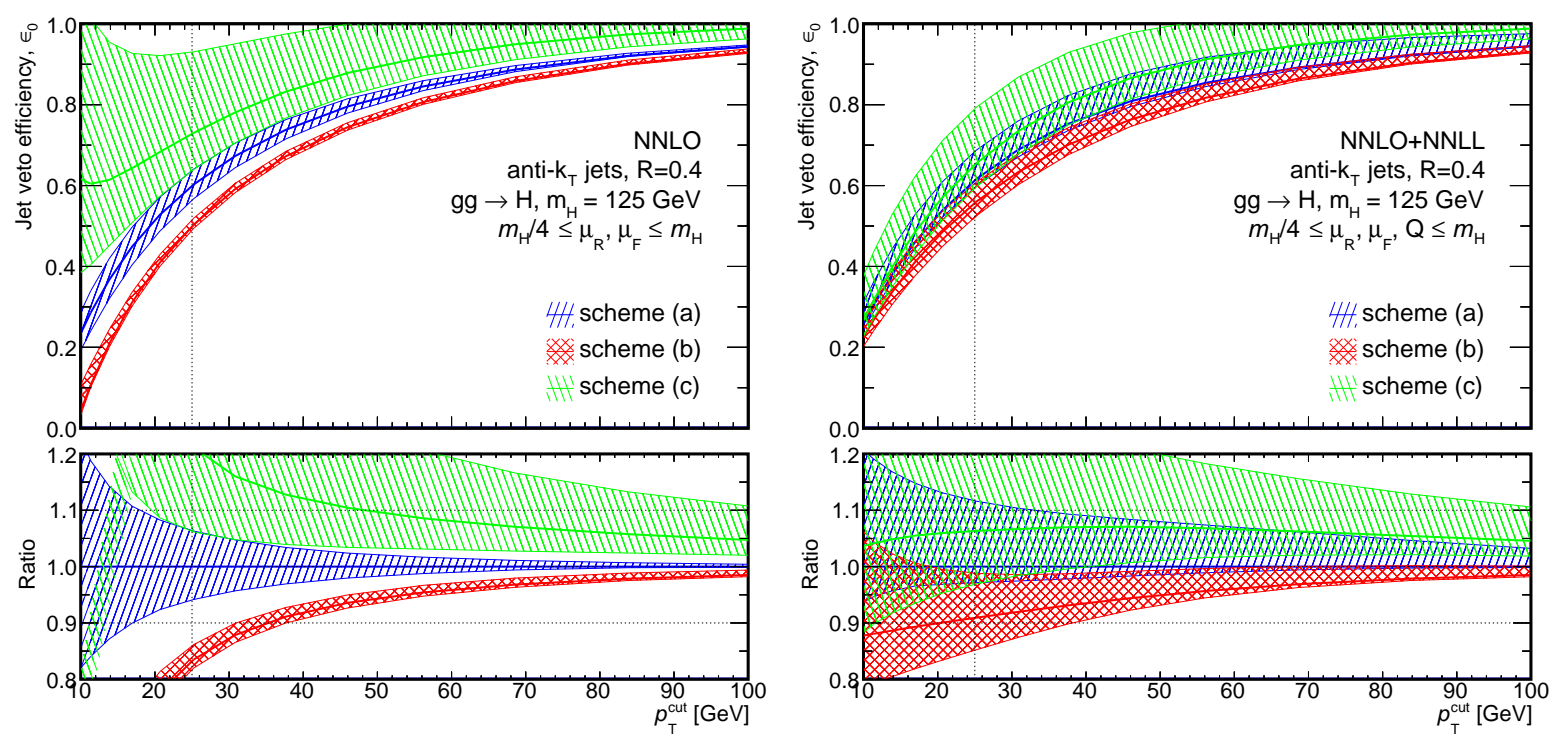

Figure 5.3: Jet veto efficiency $\epsilon_{0}$ versus the jet $p_{\mathrm{T}}$ threshold, computed with fixed order (left) and resummed (right) calculations by JeTVHeTo [127]. The bands show scale uncertainties. The bands of schemes (b) and (c) are not used in $\Delta \epsilon_{0}$.

Figure 5.3 shows how scheme differences of $\epsilon_{0}$ inflate the perturbative uncertainties compared to scale variations of $\epsilon_{0}^{(\mathrm{a})}$. At $p_{\mathrm{T}}^{\text {cut }}=25 \mathrm{GeV}$, it increases $\Delta \epsilon_{0}$ from $\sim 5 \%$ to $\sim 20 \%$. Figure 5.3 also shows how resummation of the $\ln \left(p_{\mathrm{T}}^{\text {cut }} / m_{H}\right)$ logarithms to all orders of $\alpha_{\mathrm{S}}$ can improve the estimation of $\epsilon_{0}$, resulting in better agreement between schemes and consequently reducing $\Delta \epsilon_{0}$. This resummation includes NNLL terms and is performed by JетVHeто [127].

The three NNLO schemes (5.18), (5.19) and (5.20) can also be used to define $\epsilon_{1}$. This offers an improvement compared to the CI prescription, which is currently limited to using $\sigma_{\geq 1}^{\mathrm{NLO}}$ and $\sigma_{\geq 2}^{\mathrm{LO}}$ (see Section 5.1.2). Unfortunately, it is not possible to calculate $\epsilon_{1}^{(\mathrm{a})}$ since a full $\sigma_{\geq 1}^{\mathrm{NNLO}}$ calculation is not yet available. Instead, we choose $\epsilon_{1}=\left(\epsilon_{1}^{(\mathrm{b})}+\epsilon_{1}^{(\mathrm{c})}\right) / 2$ and $\Delta \epsilon_{1}$ is evaluated by an envelope of scale uncertainties in both $\epsilon_{1}^{(\mathrm{b})}$ and $\epsilon_{1}^{(\mathrm{c})}$, which are calculated using MCFM [128]. The validity of this approximation is tested using $g g$-initiated diagrams only, for which a $\sigma_{\geq 1}^{\mathrm{NNLO}}$ calculation exists [129]. For $k_{\mathrm{T}}$ jets with $R=0.5$ and $p_{\mathrm{T}}^{\text {cut }}=30 \mathrm{GeV}$, we find that $\epsilon_{1}^{(\mathrm{a})}=0.831, \epsilon_{1}^{(\mathrm{b})}=0.761$ and $\epsilon_{1}^{(\mathrm{c})}=0.843$.

The NNLO+NNLL $(\mathrm{QCD})+\mathrm{NLO}(\mathrm{EW}) \sigma_{\text {tot }}$ calculation, the JeTVHETo NNLO+NNLL $\epsilon_{0}$ calculation and the MCFM fixed order $\epsilon_{1}$ calculation are used as inputs to the JVE prescription (5.17). Table 5.2 shows the jet-binned cross sections and uncertainties obtained using simple Gaussian propagation of uncertainties, though the nuisance parameters are constrained by log-normal distributions in the statistical model (see Section 8.2.2). 


\begin{tabular}{lcc}
\hline & $x$ & $\Delta x / x$ \\
\hline$\sigma_{\text {tot }}(\mathrm{pb})$ & $19.27 \pm 1.50$ & $7.8 \%$ \\
$\epsilon_{0}$ & $0.613 \pm 0.072$ & $11.7 \%$ \\
$\epsilon_{1}$ & $0.615 \pm 0.061$ & $9.9 \%$ \\
\hline$\sigma_{0}(\mathrm{pb})$ & $11.81 \pm 1.66$ & $14.1 \%$ \\
$\sigma_{1}(\mathrm{pb})$ & $4.59 \pm 1.03$ & $22.4 \%$ \\
$\sigma_{\geq 2}(\mathrm{pb})$ & $2.87 \pm 0.73$ & $25.4 \%$ \\
\hline
\end{tabular}

Table 5.2: Results of the jet veto efficiency prescription for ggF, with $m_{H}=125 \mathrm{GeV}$ and $\sqrt{s}=8 \mathrm{TeV}$. $\sigma_{\text {tot }}$ is from reference [10], $\epsilon_{0}$ is from JetVHeto and $\epsilon_{1}$ is from MCFM.

Figure 5.4 compares the above $\epsilon_{0}$ and $\epsilon_{1}$ calculations to a variety of different PowHEGBox+Pythia 8 configurations. The $\epsilon_{N}$ calculations are performed at parton-level in the large- $m_{t}$ limit; the solid lines show similar configurations of PowhegBox+PYThia 8, and can be considered directly comparable. The effect of consecutively adding hadronisation, MPI and finite quark mass effects are also shown. Finally, the blue line shows how reweighting the Higgs boson $p_{\mathrm{T}}$ distribution (see Section 5.2.1) influences these observables. Figure 5.4 shows that, following $p_{\mathrm{T}, H}$ reweighting, the $\mathrm{MC}$ is within the perturbative uncertainty band for both $\epsilon_{0}$ and $\epsilon_{1}$.

\subsubsection{Discussion of results}

It is helpful to directly compare the predicted jet-binned cross sections of the two prescriptions, as in Figure 5.5. "Fixed order CI" and "resummed JVE" are the prescriptions described in the preceding sections. JVE offers a reduction in uncertainty compared to CI, whilst both prescriptions remain consistent with the PowhegBox+Pythia 8 MC used, both before and after $p_{\mathrm{T}, H}$ reweighting. The improvement is due to the resummation of large logarithms in $\epsilon_{0}$ and including higher order terms in $\epsilon_{1}$. The JVE prescription is chosen to estimate the perturbative uncertainties in the jet binning.

"Fixed order JVE" replaces the resummed $\epsilon_{0}$ calculation with the fixed order $\epsilon_{0}$ calculation (i.e. replace the red band with the green band in Figure 5.4). This has the same formal accuracy as "fixed order CI" when calculating $\sigma_{0}$, and so their comparison 

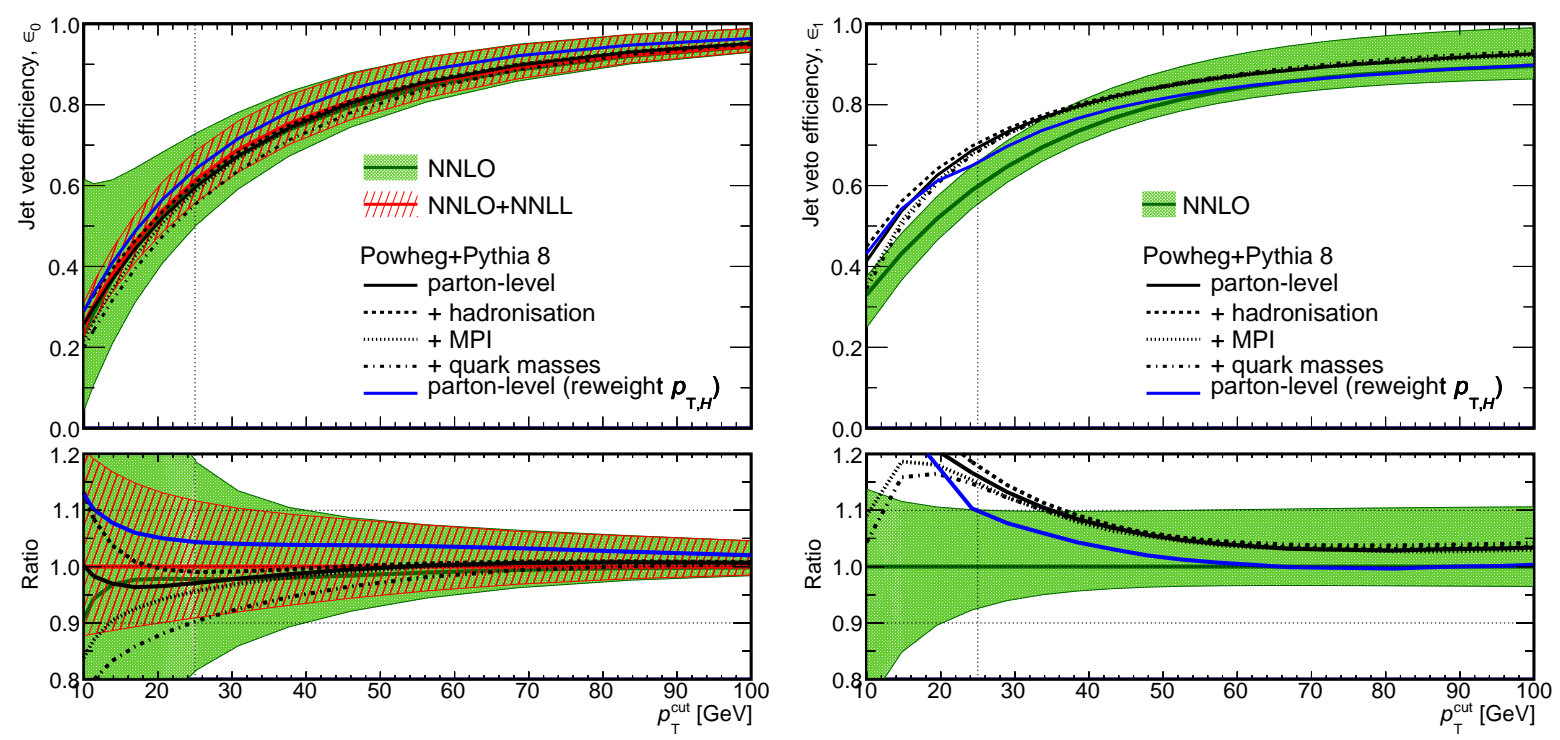

Figure 5.4: Veto efficiencies of a first jet (left) and a second jet (right), versus the jet $p_{\mathrm{T}}$ threshold. Fixed order (green) and resummed (red) results are shown with their perturbative uncertainties, and are compared to different MC scenarios.

in the 0 -jet bin directly evaluates how conservative each prescription is. JVE is found to be more conservative than CI.

Recently, a more general prescription for evaluating perturbative uncertainties in jetbinned cross sections has been proposed, to directly estimate each uncertainty amplitude in (5.9) [123]. Although it offers a further reduction in uncertainty, this prescription is not considered here. It is not viable as it predicts $\sigma_{\text {tot }}$ to be $\sim 10 \%$ larger than that presented in reference [10], which is universally used by the ATLAS and CMS collaborations for analyses of the Run I dataset. Additionally, it includes incomplete higher order terms in the perturbative series, and employs a controversial $\pi^{2}$ resummation technique. 


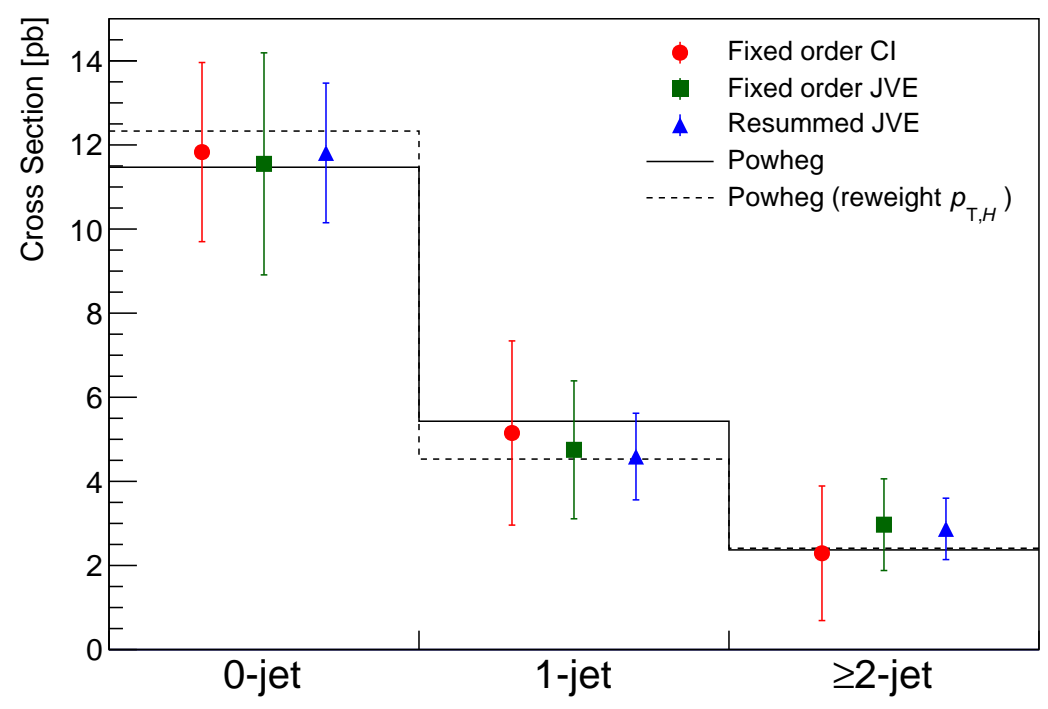

Figure 5.5: Jet-binned cross sections for ggF production with $m_{H}=125 \mathrm{GeV}$ and $\sqrt{s}=8 \mathrm{TeV}$. The error bars show the perturbative uncertainty associated with each prescription. Consistency with PowhegBox+Pythia 8 is also shown, normalised to $19.27 \mathrm{pb}$.

\subsection{Monte Carlo modelling}

The ggF signal is modelled by PowhegBox+Pythia 8, including the exact mass dependence of the $t$ and $b$ quarks in the loop [130], and using the CT10 PDF [62] to describe the incoming partons. Various aspects of this modelling are now discussed.

\subsubsection{Higgs boson transverse momentum}

The MC events are produced with the PowHEGBox parameter hfact tuned to $m_{H} / 1.2 .^{5}$ This setting reproduces the NNLO+NNLL $p_{\mathrm{T}, H}$ distribution calculated by HQT 2.0 [131] in the large- $m_{t}$ limit, when effects of hadronisation, MPI and finite quark masses are turned off in PowHEgBox+PythiA 8.

The $p_{\mathrm{T}, H}$ distribution of the generated MC events is reweighted to the best available prediction. ${ }^{6}$ Simply reweighting the inclusive $p_{\mathrm{T}, H}$ distribution is found to underestimate

\footnotetext{
${ }^{5}$ hfact controls the scale at which the first emission transitions from Sudakov-like to ME-like. Its use in tuning $p_{\mathrm{T}, H}$ is discussed in Sections 4.5 and 4.9 of reference [43].

6 This $p_{\mathrm{T}, H}$ reweighting is motivated by the $H \rightarrow \gamma \gamma$ and $H \rightarrow \tau \tau$ analyses, which feature boosted Higgs boson selection categories.
} 
$\sigma_{\geq 2}$ (c.f. Section 5.1), due to correlations between $p_{\mathrm{T}, H}$ and $N_{\text {jets }}$. Thus, the reweighting is required to simultaneously satisfy three criteria:

- the inclusive $p_{\mathrm{T}, H}$ distribution agrees with HRES 2.1 [132],

- the $p_{\mathrm{T}, H}$ distribution in the $\geq 2$-jet bin agrees with the $g g \rightarrow H j j$ process calculated by MiNLO+PYThia 8 [133],

- and the jet-binned cross sections agree with Section 5.1.

HRES 2.1 computes the inclusive $p_{\mathrm{T}, H}$ distribution with NNLO+NNLL accuracy and includes finite $m_{t}$ and $m_{b}$ effects in the loop. It also employs a dynamic scale of $\mu_{0}=\sqrt{m_{H}^{2}+p_{\mathrm{T}, H}^{2}}$ as the nominal $\mu_{\mathrm{R}}$ and $\mu_{\mathrm{F}}$ scale, which improves results at high $p_{\mathrm{T}, H}$ compared to the fixed $\mu_{0}=m_{H}$ scale used by HQT 2.0. MiNLO is an improved version of PowhegBox, which includes higher order logarithmic contributions through the careful choice of $\mu_{\mathrm{R}}$ and $\mu_{\mathrm{F}}$ scales [133]. Figure 5.5 shows that the reweighting preserves agreement with the predicted $N_{\text {jets }}$ distribution.

\subsubsection{Event selection acceptance}

In order to measure the total ggF cross section, the signal acceptance of the object and event selections must be estimated. The extrapolation from the measured phase space to the inclusive phase space introduces uncertainties. It is helpful to separate theoretical uncertainties from the others by measuring an intermediate cross section in a fiducial region of phase space, as defined in Table 5.3 for each signal region. Events featuring leptonic $\tau$ decays are excluded from the fiducial region, in order to make it easier for theorists to calculate the expected fiducial cross section within their model of choice. These criteria use hadron-level objects and are chosen to closely represent those of the detector-level selection, in order to minimise the extrapolation to the fiducial region.

To define the hadron-level objects, the MC event record is used to identify prompt charged leptons and neutrinos. A $\boldsymbol{E}_{\mathrm{T}, \nu \nu}$ vector is constructed from the neutrinos. Each charged lepton is 'dressed' by adding the four-momenta of photons within a cone of $\Delta R<0.1$, in order to recover energy lost via QED FSR. Jets are found using individual particles as inputs (c.f. topo-clusters at detector-level). Muons and neutrinos are excluded from jet finding since they interact weakly with the calorimeter. Objects must pass the same $p_{\mathrm{T}}, \eta$ and overlap removal criteria applied at detector-level. 


\begin{tabular}{|c|c|}
\hline Jet binning & $e \mu / \mu e$ \\
\hline \multirow[t]{6}{*}{ Inclusive } & $p_{\mathrm{T}, \ell}^{\text {lead }}>22$ and $p_{\mathrm{T}, \ell}^{\text {sublead }}>10$ \\
\hline & $m_{\ell \ell}>10 \quad m_{\ell \ell}>12$ \\
\hline & $\left|m_{\ell \ell}-m_{Z}\right|>15$ \\
\hline & $p_{\mathrm{T}, \nu \nu}>20 \quad E_{\mathrm{T}, \mathrm{rel}, \nu \nu}>40$ \\
\hline & $m_{\ell \ell}<55$ \\
\hline & $\Delta \phi(\ell, \ell)<1.8$ \\
\hline \multirow[t]{2}{*}{0 -jet } & \multirow{2}{*}{$\begin{array}{c}\Delta \phi\left(\ell \ell, p_{\mathrm{T}}^{\text {inv }}\right)>\pi / 2 \\
p_{\mathrm{T}, \ell \ell}>30\end{array}$} \\
\hline & \\
\hline \multirow[t]{2}{*}{1 -jet } & $\max \left(m_{\mathrm{T}, \ell}\right)>50$ \\
\hline & $m_{\tau \tau}<m_{Z}-25$ \\
\hline \multirow[t]{2}{*}{$\geq 2$-jet } & $m_{\tau \tau}<m_{Z}-25$ \\
\hline & Fail $\Delta y(j, j)>3.6$ or $m_{j j}>600$ or CJV or OLV \\
\hline
\end{tabular}

Table 5.3: Hadron-level event selection criteria for each fiducial region. Events featuring leptonic $\tau$ decays are excluded from the fiducial region. Cuts on energy, momentum and mass are given in $\mathrm{GeV}$, and angular cuts are given in radians. The CJV and OLV are the central jet veto and outside lepton veto, respectively. See Chapter 4 for a detailed explanation of the criteria.

The measured fiducial cross section is extracted by

$$
\sigma_{\mathrm{ggF}}^{\mathrm{fid}}=\frac{N_{\mathrm{obs}}-N_{\mathrm{bkg}}}{C_{\mathrm{ggF}} \cdot L}
$$

where $N_{\text {obs }}$ is the observed number of events, $N_{\mathrm{bkg}}$ is the expected number of background events, $L$ is the luminosity, and $C_{\mathrm{ggF}}$ is the ratio of the expected number of $g g \mathrm{~F}$ events passing the detector-level selection to those passing the fiducial selection. $C_{\mathrm{ggF}}$ accounts for detector effects such as lepton trigger and reconstruction efficiencies and object mismeasurement due to the finite resolution of the detector.

On the other hand, the measured total cross section is extracted by

$$
\sigma_{\mathrm{ggF}}=\frac{N_{\mathrm{obs}}-N_{\mathrm{bkg}}}{C_{\mathrm{ggF}} \cdot A_{\mathrm{ggF}} \cdot \mathrm{BR} \cdot L}
$$

where $A_{\mathrm{ggF}}$ is the ratio of the expected number of ggF events passing the fiducial selection to the total expected number of ggF events. $A_{\mathrm{ggF}}$ accounts for the acceptance of the 


\begin{tabular}{lcc}
\hline & 0 -jet & 1 -jet \\
\hline$C_{\text {ggF }}(\times 100)$ & $50.7 \pm 2.7$ & $50.6 \pm 2.2$ \\
Trigger efficiency & $0.7 \%$ & $0.6 \%$ \\
Lepton efficiency & $2.6 \%$ & $2.4 \%$ \\
Lepton $p_{\mathrm{T}}$ scale and resolution & $1.1 \%$ & $0.9 \%$ \\
Jet energy scale and resolution & $4.3 \%$ & $2.2 \%$ \\
Jet $b$-tagging efficiency & $0.0 \%$ & $1.8 \%$ \\
$p_{\mathrm{T}}^{\text {inv } \text { modelling }}$ & $0.1 \%$ & $0.1 \%$ \\
PS/UE & $1.5 \%$ & $2.0 \%$ \\
\hline$A_{\mathrm{ggF}}(\times 100)$ & $20.6 \pm 3.0$ & $7.5 \pm 1.7$ \\
Perturbative QCD & & \\
Jet binning & $14 \%$ & $22 \%$ \\
Other cuts & $1.1 \%$ & $1.4 \%$ \\
PDFs & $3.7 \%$ & $3.4 \%$ \\
PS/UE & $2.2 \%$ & $3.8 \%$ \\
NLO-PS & $1.8 \%$ & $1.1 \%$ \\
\hline$C_{\text {ggF }} \cdot A_{\text {ggF }}(\times 100)$ & $10.4 \pm 1.6$ & $3.8 \pm 0.9$ \\
\hline
\end{tabular}

Table 5.4: The signal acceptances $C_{\mathrm{ggF}}, A_{\mathrm{ggF}}$ and $C_{\mathrm{ggF}} \cdot A_{\mathrm{ggF}}$. A breakdown of the relative uncertainties from different sources is also shown.

event selection criteria. BR incorporates the branching ratios of the Higgs and $W$ bosons for the channel in question.

Fiducial cross sections are only extracted from the 0 -jet and 1-jet bins of the $e \mu / \mu e$ channels, since these are the most sensitive signal regions. The acceptances $C_{\mathrm{ggF}}, A_{\mathrm{ggF}}$ and $C_{\mathrm{ggF}} \cdot A_{\mathrm{ggF}}$ are displayed in Table 5.4, together with their respective uncertainties.

Theoretical uncertainties in the acceptance (other than the jet binning uncertainties discussed in Section 5.1) are evaluated at hadron-level by changing some aspect of the MC modelling and measuring the change in acceptance relative to the jet-binned cross sections. In the case of PDF uncertainties, the acceptance is calculated relative to the total cross section, in order to include PDF uncertainties in the jet binning. Since these uncertainties are used in extracting the signal strength, the fiducial volume is modified to include events featuring lepton $\tau$ decays. 
Four sources of theoretical uncertainty are considered:

- higher order corrections,

- PDFs,

- parton shower, hadronisation and underlying event models,

- NLO-PS matching scheme.

Uncertainties due to higher order corrections are evaluated via independent variation of renormalisation and factorisation scales in the range $m_{H} / 2 \leq \mu_{\mathrm{R}}, \mu_{\mathrm{F}} \leq 2 m_{H}$, whilst observing the constraint $1 / 2 \leq \mu_{\mathrm{R}} / \mu_{\mathrm{F}} \leq 2$. In the $\geq 2$-jet bin, this is evaluated using NLO MC of the $g g \rightarrow H+1$ jet process, since the NLO MC of the inclusive $g g \rightarrow H$ process relies upon the parton shower to model the second jet.

Uncertainties due to PDFs are evaluated in two ways. First, the acceptance is compared to that predicted with the MSTW2008 PDF [58]. Second, the set of PDF eigenvectors corresponding to 90\% CL of the CT10 fit were used to evaluate an uncertainty, which was then rescaled to $68 \% \mathrm{CL}$ (assuming a Gaussian distribution). PDF uncertainties are evaluated using MC@NLO.

Uncertainties due to the parton shower (PS), hadronisation and underlying event (UE) models are evaluated by comparing PowhegBox showered by PYTHIA 8 (nominal), Pythia 6 and Herwig. Uncertainties due to the NLO-PS matching scheme are evaluated by comparing PowhegBox+Herwig to MC@NLO+HerwiG++.

Theoretical acceptance uncertainties are also calculated for every signal region used in the fitting procedure, including the individual signal regions split by $p_{\mathrm{T}, \ell}^{\text {sublead }}$ and $m_{\ell \ell}$. These uncertainties are shown in Table 5.5, and are evaluated in the fiducial regions described in Table 5.3. 


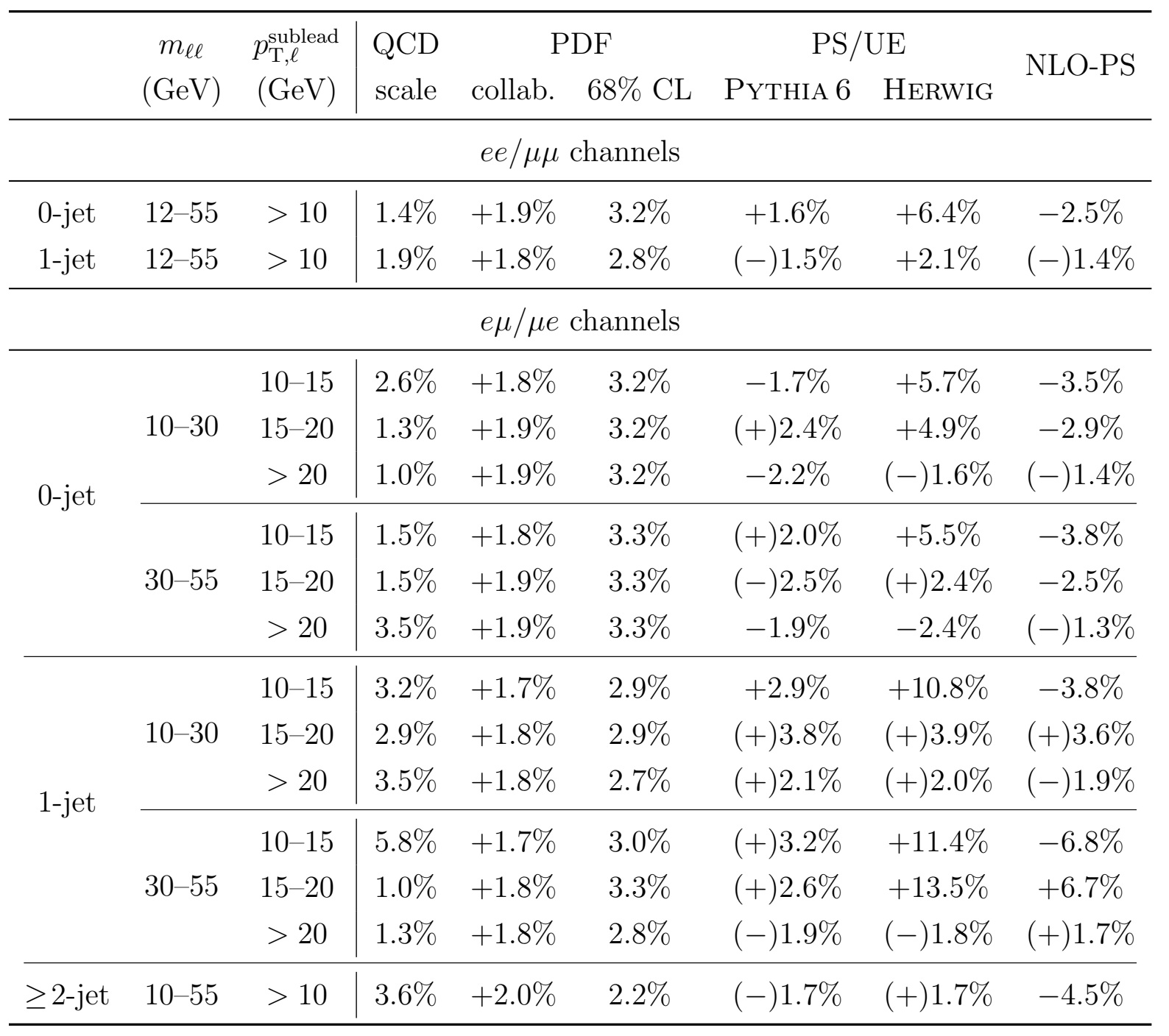

Table 5.5: Theoretical uncertainties in the ggF acceptance for each signal region used in the fitting procedure. PDF uncertainties are in acceptances relative to the inclusive cross section, whereas others are calculated within jet bins. When the uncertainty is statistically insignificant, the statistical uncertainty on the generator difference is given, and the sign of the generator difference is parenthesised. These are the uncertainties used in the fit. 


\subsection{3 $\mathrm{m}_{\mathrm{T}}$ shape modelling}

Theoretical uncertainties in the shape of the $m_{\mathrm{T}}$ distribution are also investigated. Uncertainties due to scale, PS/UE and NLO-PS choices are considered using the methods described above (PDF uncertainties are neglected). The split signal regions are not used in this study since the statistical fluctuations in the $m_{\mathrm{T}}$ distributions are large.

Each uncertainty is parametrised by fitting the ratio of the $m_{\mathrm{T}}$ shapes, and then symmetrising the fit to produce "up" and "down" variations. The $m_{\mathrm{T}}$ distributions are normalised to unit integral in order to remove effects from acceptance uncertainties. In cases where multiple variations exist within a single uncertainty source (such as the six scale variations), the largest deviation from the nominal result is fit. A linear fit is used in the central $m_{\mathrm{T}}$ region, and a constant is used in the low- $m_{\mathrm{T}}$ and high- $m_{\mathrm{T}}$ tails of the distribution where statistical fluctuations dominate.

These fits allow the hadron-level $m_{\mathrm{T}}$ distribution of the ggF signal to be reweighted to the "up" and "down" variations. In this way, the $m_{\mathrm{T}}$ shape uncertainty is treated as a nuisance parameter in the $H \rightarrow W W$ fitting procedure. The uncertainties for the 0 -jet and 1-jet signal regions are displayed in Figure 5.6. 

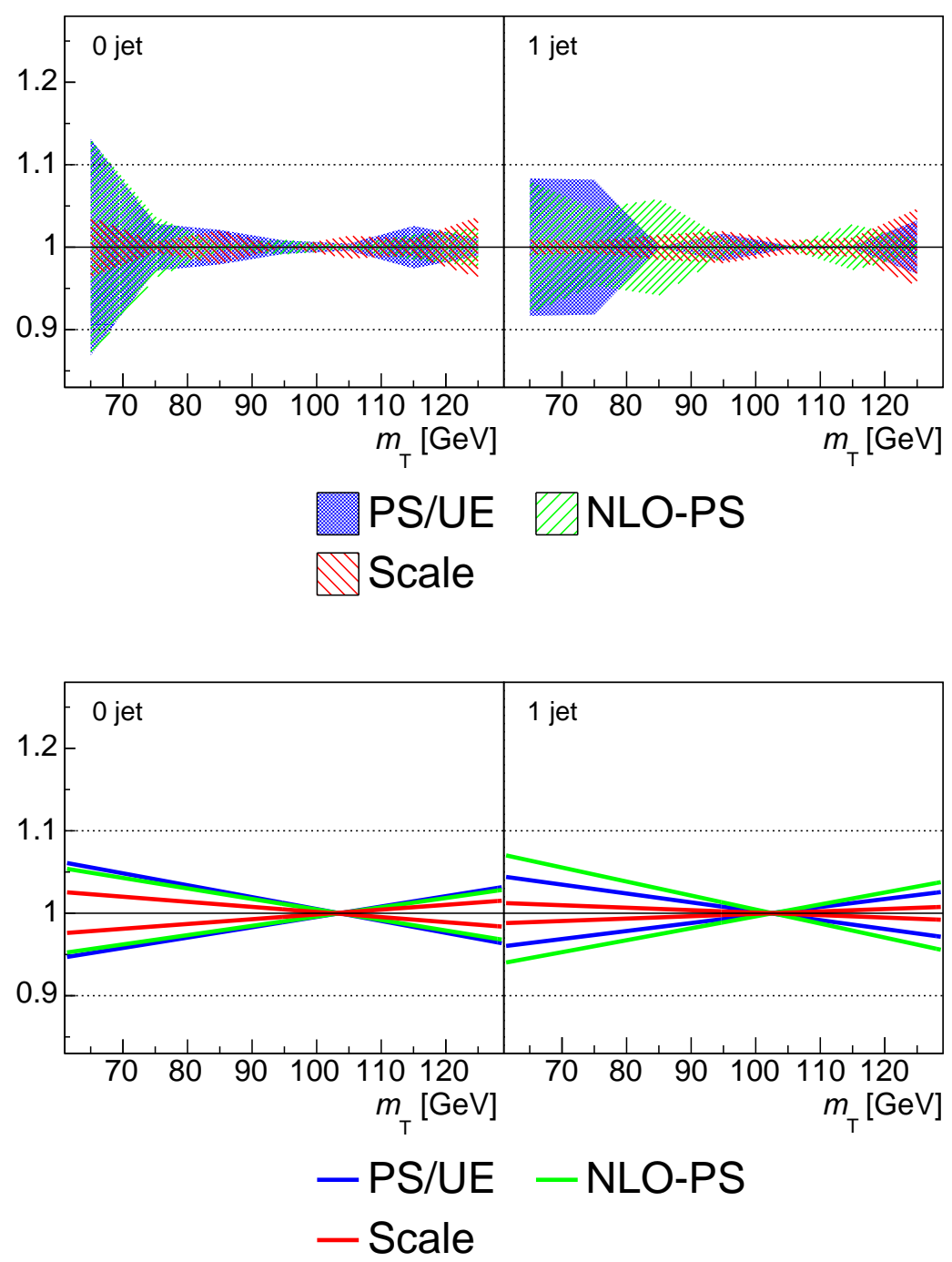

Figure 5.6: $g g F m_{\mathrm{T}}$ shape systematic uncertainties in the 0-jet and 1-jet signal regions of the $e \mu / \mu e$ channels. The raw uncertainties are shown above and the fits are shown below. The constant (flat) terms of the fit are outside the visible $x$-range. 


\section{Chapter 6}

\section{WW measurement and modelling}

Background modelling is of paramount importance to the $H \rightarrow W W$ search. As an irreducible background, continuum $W W \rightarrow \ell \nu \ell \nu$ production dominates the 0 -jet and 1-jet bins, where the majority of the sensitivity lies. Thus, even a small uncertainty in this background can have a large effect on the uncertainty in the measured signal. The process is also of general interest to electroweak phenomenology and is sensitive to anomalous triple gauge couplings (aTGCs), as seen in Figure 6.1.

Section 6.1 describes a dedicated $W W$ cross section measurement performed using the 2011 dataset of $p p$ collisions at $\sqrt{s}=7 \mathrm{TeV}$. Then, Section 6.2 describes how the $W W$ background is estimated in the phase space of the $H \rightarrow W W$ search.
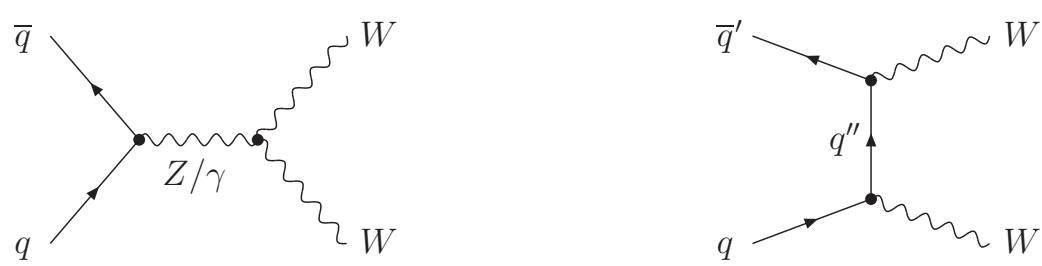

Figure 6.1: LO Feynman diagrams for $W W$ production, for the $s$-channel (left) and $t$-channel (right). The $s$-channel contains a triple gauge coupling vertex. 


\subsection{Cross section measurement in the 0 -jet bin}

This section describes the $W W$ cross section measurement using the $\sqrt{s}=7 \mathrm{TeV}$ dataset, published in reference [15]. As the experimental signature is the same as $H \rightarrow W W$, many aspects are shared. However, there are two key differences between the analyses:

1. The analysis described in Chapter 4 is optimised for low mass $\left(m_{H} \approx 125 \mathrm{GeV}\right)$ resonant $W W$ production. It therefore requires at least one $W$ boson to be off-shell, and consequently employs low lepton $p_{\mathrm{T}}$ thresholds $\left(p_{\mathrm{T}}>10 \mathrm{GeV}\right)$. Conversely, the measurement of non-resonant $W W$ production is optimised for on-shell $W$ bosons, and raises the lepton thresholds to $p_{\mathrm{T}}>20 \mathrm{GeV}$. This reduces hadrons misidentified as leptons, and obviates the need to split the different-flavour channel into $e \mu / \mu e$.

2. The total $W W$ cross section at $\sqrt{s}=7 \mathrm{TeV}$ is $\sigma_{\mathrm{NLO}}=44.7_{-1.9}^{+2.1} \mathrm{pb}$, which is much larger than that of $g g \rightarrow H \rightarrow W W\left(3.3 \pm 0.4 \mathrm{pb}\right.$ for $\left.m_{H}=125 \mathrm{GeV}\right)$. This enables backgrounds to be suppressed by tighter criteria, whilst retaining a large number of signal events. As an example, only the 0 -jet bin is used.

\subsubsection{Reconstruction of physics objects}

Beam conditions in 2011 were quite different to 2012; in particular, the pile-up environment was considerably less harsh (see Figure 3.2b). Consequently, the reconstruction of physics objects is slightly different to that described in Section 4.2:

\section{Electrons}

The Gaussian Sum Filter was not implemented in the 2011 reconstruction, and so the efficiency is lower (see Figure 4.3). To reduce fakes, the cut-based tight identification criteria are used. The reduced pile-up allows tighter calorimeter isolation to be applied, $E_{\mathrm{T}}^{\text {cone }}(0.3) / E_{\mathrm{T}}<0.14$, and this in turn allows for looser tracker isolation $p_{\mathrm{T}}^{\text {cone }}(0.3) / E_{\mathrm{T}}<0.13$. Finally, the association with the primary vertex is relaxed, with the transverse impact parameter $d_{0}$ required to be within ten standard deviations of zero. The $p_{\mathrm{T}}$ threshold is raised to $20 \mathrm{GeV}$.

\section{Muons}

Differences to muon reconstruction are minimal. Slightly tighter quality criteria are applied to the ID tracks. The isolation criteria are $E_{\mathrm{T}}^{\text {cone }}(0.3) / p_{\mathrm{T}}<0.14$ and $p_{\mathrm{T}}^{\text {cone }}(0.3) / p_{\mathrm{T}}<0.15$. The $p_{\mathrm{T}}$ threshold is raised to $20 \mathrm{GeV}$. 


\begin{tabular}{llc}
\hline & EF_e20_medium & 14th Apr -4 th Aug \\
$e$ & EF_e22_medium & 4th Aug - 22nd Aug \\
& EF_e22vh_medium1 & 7th Sep - 30th Oct \\
\hline \multirow{2}{*}{$\mu$} & EF_mu18_MG & 14th Apr - 29th Jul \\
& EF_mu18_MG_medium & 30th Jul - 30th Oct \\
\hline
\end{tabular}

Table 6.1: Single lepton triggers employed in the $2011 W W$ cross section measurement. Trigger names are explained in Table 4.4.

Jets

A lower pile-up noise threshold is used in topo-clustering, corresponding to $\mu=8$ (c.f. $\mu=30$ in 2012). Local cluster weighting (LCW) is not performed on topo-clusters, and so jets are corrected directly from the EM scale to the Jet Energy Scale (JES). In 2011, the pile-up subtraction step of the calibration is less sophisticated, and is averaged over $N_{\mathrm{PV}}$ and $\mu$ rather than an event-by-event correction [134]. The jet vertex fraction criterion is also removed. A unified $p_{\mathrm{T}}$ threshold of $25 \mathrm{GeV}$ is used over the entire range $|\eta|<4.5$.

\section{Missing transverse momentum}

Calorimeter-based $p_{\mathrm{T}}^{\mathrm{inv}}$ is used exclusively throughout the analysis.

\subsubsection{Event selection criteria}

As mentioned above, the event selection of the $W W$ measurement can be tighter than that of the $H \rightarrow W W$ search, and does not require such stringent optimisation.

\section{Data quality}

The $2011 p p$ dataset (see Section 3.2.2) is subject to data quality criteria as in 2012, though some criteria are specific to the data-taking conditions of 2011 . The selected dataset corresponds to an integrated luminosity of $4.64 \pm 0.18 \mathrm{fb}^{-1}$.

\section{Trigger}

The lowest unprescaled single lepton triggers were used to support $p_{\mathrm{T}, \ell}^{\text {lead }}>25 \mathrm{GeV}$ in the offline analysis, whilst operating on the plateau. These changed throughout the year as beam conditions changed, and are displayed in Table 6.1. 


\begin{tabular}{cc}
\hline$e \mu$ & $e e / \mu \mu$ \\
\hline$p_{\mathrm{T}, \ell}^{\text {lead }}>25$ and $p_{\mathrm{T}, \ell}^{\text {sublead }}>20$ \\
$m_{\ell \ell}>10$ & $m_{\ell \ell}>15$ \\
- & $\left|m_{\ell \ell}-m_{Z}\right|>15$ \\
$E_{\mathrm{T}, \text { rel }}^{\text {miss }}>25$ & $E_{\mathrm{T}, \text { rel }}^{\text {miss }}>45$ \\
\multicolumn{3}{c}{$N_{\text {jets }}=0$} \\
$p_{\mathrm{T}, \ell \ell}>30$ \\
\hline
\end{tabular}

Table 6.2: Summary of $W W$ event selection. Cuts are given in $\mathrm{GeV}$. The relevant observables are described in Chapter 4.

\section{Event selection}

The event selection is shown in Table 6.2 and is similar to the 0 -jet $H \rightarrow W W$ selection in Table 4.5, although the topological cuts of the Higgs boson decay are obviously not applied. The major differences are the raised lepton thresholds, the raised $p_{\mathrm{T}}^{\text {inv }}$ cuts, and the lack of $\Delta \phi\left(\ell \ell, p_{\mathrm{T}}^{\text {inv }}\right)$ and $f_{\text {recoil }}$ cuts.

\subsubsection{Analysis strategy}

In contrast to the $H \rightarrow W W$ search, the signal region of the $W W$ measurement has a high signal-to-background ratio. Thus, it is sufficient to simply count the number of events passing the selection, rather than fit a discriminating observable like $m_{\mathrm{T}}$.

In order to measure the total $W W$ cross section, it is necessary to estimate the signal acceptance of the event selection (see Section 6.1.4). This extrapolation from the measured phase space to the inclusive phase space introduces theoretical uncertainties. It is helpful to separate these theoretical uncertainties from the others, by measuring an intermediate cross section in a fiducial region of phase space, chosen to be similar to that used in the detector-level selection in order to minimise the extrapolation.

The fiducial region is defined by the criteria in Table 6.2 applied to hadron-level objects, which are now described. The MC event record is used to identify prompt leptons and neutrinos. An $\boldsymbol{E}_{\mathrm{T}, \nu \nu}$ vector is constructed from the neutrinos. Each lepton is 'dressed' by adding the four-momenta of photons within a cone of $\Delta R<0.1$, in order to recover energy lost via QED FSR. Jets are found using particle four-momenta as inputs 
(c.f. topo-clusters at detector-level). Muons and neutrinos are excluded from jet finding since they interact weakly with the calorimeter. Objects must pass the same $p_{\mathrm{T}}, \eta$ and overlap-removal criteria applied at detector-level.

The fiducial cross section is extracted from measurements using

$$
\sigma_{W W}^{\mathrm{fid}}=\frac{N_{\mathrm{obs}}-N_{\mathrm{bkg}}}{C_{W W} \cdot L}
$$

where $N_{\text {obs }}$ is the observed number of events passing the event selection, $N_{\mathrm{bkg}}$ is the expected number of background events (see Section 6.1.5), $L$ is the luminosity of the dataset, and $C_{W W}$ is the ratio of the expected number of signal events passing the detector-level selection to those passing the fiducial selection. $C_{W W}$ accounts for detector effects such as trigger and reconstruction efficiencies, and object mismeasurement due to the finite resolution of the detector.

The total cross section can be extracted using

$$
\sigma_{W W}=\frac{N_{\mathrm{obs}}-N_{\mathrm{bkg}}}{C_{W W} \cdot A_{W W} \cdot \mathrm{BR} \cdot L}
$$

where $A_{W W}$ is the ratio of the expected number of signal events passing the fiducial selection to the total expected number of signal events. $A_{W W}$ accounts for the signal acceptance of the event selection criteria. BR incorporates the branching ratios of both $W$ bosons for the channel in question, and includes contributions from leptonic $\tau$ decays.

It is apparent from (6.1) and (6.2) that both signal and background modelling are important inputs in measuring a cross section.

\subsubsection{Signal modelling}

The $W W$ process is modelled at NLO by MC@NLO+Herwig. The NNLO $g g \rightarrow W W$ diagrams contribute $\sim 3 \%$ to the total cross section, due to the large gluon luminosities at the LHC, and are modelled by GG2Ww+HeRwiG [135]. The signal acceptances $C_{W W}$ and $A_{W W}$ are corrected for the small mismodelling of trigger and lepton reconstruction efficiencies, through in situ tag-and-probe studies (see Section 4.2.3).

The jet veto in the event selection is responsible for the dominant uncertainties in both $C_{W W}$ and $A_{W W}$. Uncertainties in the jet energy scale (JES) and jet energy resolution (JER) lead to large uncertainties in $C_{W W}$, since the leading jet $p_{\mathrm{T}}$ is a rapidly 


\begin{tabular}{c|cc|cc}
\hline \multirow{2}{*}{\begin{tabular}{c|c|c} 
Nontribution to correction factor \\
Exper.
\end{tabular}} & Theor. & \multicolumn{2}{c}{$\begin{array}{c}\text { Jet veto correction factor } \\
\text { Exper. }\end{array}$} & Theor. \\
\hline$C_{W W}$ & $\Delta \epsilon_{W W}^{\mathrm{MC}}$ & - & $\Delta\left(\epsilon_{W W}^{\mathrm{MC}} / \epsilon_{Z}^{\mathrm{MC}}\right)$ & $\Delta \epsilon_{Z}^{\mathrm{MC}}$ \\
$A_{W W}$ & - & $\Delta \epsilon_{W W}^{\mathrm{MC}}$ & - & $\Delta \epsilon_{W W}^{\mathrm{MC}}$ \\
$C_{W W} \cdot A_{W W}$ & $\Delta \epsilon_{W W}^{\mathrm{MC}}$ & $\Delta \epsilon_{W W}^{\mathrm{MC}}$ & $\Delta\left(\epsilon_{W W}^{\mathrm{MC}} / \epsilon_{Z}^{\mathrm{MC}}\right)$ & $\Delta\left(\epsilon_{W W}^{\mathrm{MC}} / \epsilon_{Z}^{\mathrm{MC}}\right)$ \\
\hline
\end{tabular}

Table 6.3: Summary of how the experimental and theoretical uncertainties on the jet veto acceptance $\epsilon$ contribute to $C_{W W}, A_{W W}$ and their product. Strategies with and without the jet veto correction factor are considered.

falling distribution. As discussed in Section 5.1, restricting QCD emissions via a jet veto introduces large theoretical uncertainties to $A_{W W}$, though these are expected to be smaller in $W W$ than in ggF since the perturbative corrections are smaller.

For these reasons, a data-driven correction factor is included within $C_{W W}$ in order to improve the modelling of the jet veto acceptance $\epsilon_{W W}$. This correction factor is derived using $Z \rightarrow \ell \ell$ events. Thus, the predicted jet veto acceptance is

$$
\epsilon_{W W}^{\mathrm{pred}}=\epsilon_{W W}^{\mathrm{MC}} \cdot \frac{\epsilon_{Z}^{\mathrm{data}}}{\epsilon_{Z}^{\mathrm{MC}}} .
$$

Application of this correction factor effectively calibrates the MC generator to data (thus the same MC generator must be used to model $\epsilon_{W W}^{\mathrm{MC}}$ and $\epsilon_{Z}^{\mathrm{MC}}$ ). As shown in Table 6.3, this enables the experimental uncertainty in the jet veto acceptance to be reduced, at the expense of introducing a theoretical uncertainty to $C_{W W}$. However, in the product $C_{W W} \cdot A_{W W}$ the theoretical uncertainty is also reduced.

Correction factors $\epsilon_{Z}^{\mathrm{data}} / \epsilon_{Z}^{\mathrm{MC}}$ are measured in control regions with high $Z \rightarrow \ell \ell$ purity. Events are selected with $p_{\mathrm{T}, \ell}^{\text {lead }}>25 \mathrm{GeV}, p_{\mathrm{T}, \ell}^{\text {sublead }}>20 \mathrm{GeV}$ and $\left|m_{\ell \ell}-m_{Z}\right|<15 \mathrm{GeV}$ in the $e e$ and $\mu \mu$ channels. The correction factor for the $e \mu$ channel is taken as the average of the other two. The correction factors are 0.957, 0.954 and 0.956 for the ee, $\mu \mu$ and $e \mu$ channels respectively. Statistical uncertainties are negligible compared to the experimental and theoretical uncertainties.

Uncertainties in $\epsilon_{W W}^{\mathrm{MC}}$ and $\epsilon_{Z}^{\mathrm{MC}}$ due to the JES are evaluated by increasing and decreasing jet energies by one standard deviation, and using the average of the absolute deviations. The JES uncertainty is determined during the in situ calibration [111]. 


\begin{tabular}{|c|c|c|c|c|c|c|c|}
\hline & \multicolumn{5}{|c|}{ Sources of relative uncertainty } & \multicolumn{2}{|c|}{ Total } \\
\hline & JES & JER & Scale & $\mathrm{PDF}$ & $\mathrm{PS} / \mathrm{UE}$ & Exper. & Theor. \\
\hline \multicolumn{8}{|c|}{$e \mu$ channel } \\
\hline$\epsilon_{W W}^{\mathrm{MC}}=0.662$ & $4.6 \%$ & $2.4 \%$ & $5.3 \%$ & $1.6 \%$ & $0.5 \%$ & $5.1 \%$ & $5.6 \%$ \\
\hline$\epsilon_{Z}^{\mathrm{MC}}=0.793$ & $3.8 \%$ & $2.6 \%$ & $2.4 \%$ & $0.8 \%$ & $0.4 \%$ & $4.6 \%$ & $2.6 \%$ \\
\hline$\epsilon_{W W}^{\mathrm{MC}} / \epsilon_{Z}^{\mathrm{MC}}=0.835$ & $0.7 \%$ & $0.2 \%$ & $3.4 \%$ & $0.9 \%$ & $0.1 \%$ & $0.7 \%$ & $3.5 \%$ \\
\hline \multicolumn{8}{|c|}{ ee channel } \\
\hline$\epsilon_{W W}^{\mathrm{MC}}=0.652$ & $4.8 \%$ & $2.0 \%$ & $5.3 \%$ & $1.6 \%$ & $0.5 \%$ & $5.2 \%$ & $5.6 \%$ \\
\hline$\epsilon_{Z}^{\mathrm{MC}}=0.798$ & $3.7 \%$ & $2.4 \%$ & $2.4 \%$ & $0.8 \%$ & $0.4 \%$ & $4.4 \%$ & $2.6 \%$ \\
\hline$\epsilon_{W W}^{\mathrm{MC}} / \epsilon_{Z}^{\mathrm{MC}}=0.817$ & $1.1 \%$ & $0.4 \%$ & $3.4 \%$ & $0.9 \%$ & $0.1 \%$ & $1.2 \%$ & $3.5 \%$ \\
\hline \multicolumn{8}{|c|}{$\mu \mu$ channel } \\
\hline$\epsilon_{W W}^{\mathrm{MC}}=0.655$ & $4.8 \%$ & $2.1 \%$ & $5.3 \%$ & $1.6 \%$ & $0.5 \%$ & $5.2 \%$ & $5.6 \%$ \\
\hline$\epsilon_{Z}^{\mathrm{MC}}=0.788$ & $4.0 \%$ & $2.8 \%$ & $2.4 \%$ & $0.8 \%$ & $0.4 \%$ & $4.9 \%$ & $2.6 \%$ \\
\hline$\epsilon_{W W}^{\mathrm{MC}} / \epsilon_{Z}^{\mathrm{MC}}=0.831$ & $0.8 \%$ & $0.7 \%$ & $3.4 \%$ & $0.9 \%$ & $0.1 \%$ & $1.0 \%$ & $3.5 \%$ \\
\hline
\end{tabular}

Table 6.4: Relative uncertainties in the $W W$ and $Z$ jet veto acceptances, and in their ratio. Results for the $e e, \mu \mu$ and $e \mu$ channels are shown separately, with $\epsilon_{Z}^{\mathrm{MC}}$ for the $e \mu$ channel taken as the average of the other two.

Similarly, uncertainties due to the JER are evaluated by increasing the JER by one standard deviation. The JER uncertainty is determined using other in situ techniques [136]. These uncertainties are displayed in Table 6.4.

Theoretical uncertainties are evaluated at hadron-level by changing some aspect of the MC modelling and measuring the effect upon the jet veto acceptance $\epsilon$. These uncertainties are displayed in Table 6.4, and their estimation is described below.

Uncertainties due to higher order corrections are evaluated using the combined inclusive method described in Section 5.1.2. The renormalisation and factorisation scales are independently varied in the range $\mu_{0} / 2 \leq \mu_{\mathrm{R}}, \mu_{\mathrm{F}} \leq 2 \mu_{0}$, where $\mu_{0}$ is the default scale for the process in question ${ }^{1}$, whilst observing the constraint $1 / 2 \leq \mu_{\mathrm{R}} / \mu_{\mathrm{F}} \leq 2$. The largest deviation is used as the uncertainty.

${ }^{1}$ The default scales used by MC@NLO are determined by $\mu_{0}^{2}=m_{\ell \ell}^{2}+p_{\mathrm{T}, \ell \ell}^{2}$ for $Z$ production and $\mu_{0}^{2}=\left(m_{\ell \nu}^{2}+p_{\mathrm{T}, \ell \nu}^{2}+m_{\ell^{\prime} \nu^{\prime}}^{2}+p_{\mathrm{T}, \ell^{\prime} \nu^{\prime}}^{2}\right) / 2$ for $W W$ production. 
Uncertainties due to parton distribution functions (PDFs) are evaluated in two ways, and added in quadrature. The jet veto acceptance predicted with the CT10 PDF is compared to that predicted with the MSTW2008 PDF [58]. The set of PDF eigenvectors corresponding to $90 \%$ CL of the CT10 fit are also used.

Uncertainties due to the parton shower (PS), hadronisation and underlying event (UE) models are evaluated by recomputing the jet veto acceptances with PowHEGBox events, and comparing the result when showered by HeRwig and by PYTHIA 6 .

By substituting uncertainties from Table 6.4 into the two strategies outlined in Table 6.3 , it is clear that the jet veto correction factor offers a significant reduction in uncertainty. Considering the $\sigma_{W W}$ extraction from the $e \mu$ channel, the contribution of the experimental uncertainty in $\epsilon_{W W}$ reduces from $5.1 \%$ to $0.7 \%$, while the theoretical uncertainty reduces from $5.6 \%$ to $3.5 \%$. However, it does introduce a theoretical uncertainty to $\sigma_{W W}^{\mathrm{fid}}$ of $2.6 \%$.

The signal acceptances $A_{W W}, C_{W W}$ and $C_{W W} \cdot A_{W W}$ are displayed in Table 6.5. The $\mu \mu$ channel has the best reconstruction efficiency and the event selection of the $e \mu$ channel has the highest yield. The uncertainties arising from different systematic sources are also shown, with those associated with the jet veto separated for clarity. The JES and JER uncertainties in the jet veto acceptance are approximated to be uncorrelated with those of the other cuts. The same is true of the theoretical uncertainties in $A_{W W}$.

\subsubsection{Background modelling}

The background estimation techniques of the $H \rightarrow W W$ search are more sophisticated than those of the $W W$ measurement at $\sqrt{s}=7 \mathrm{TeV}$. For this reason, the techniques used in the $W W$ measurement are only described briefly here, whereas those of the $H \rightarrow W W$ search shall be discussed in detail in Section 6.2 and Chapter 7 .

Although many backgrounds are modelled by a data-driven technique, there is often some underlying dependence upon MC. For this reason, Table 6.6 states the MC generators used to model each process. 


\begin{tabular}{lcccc}
\hline & $e \mu$ & $e e$ & $\mu \mu$ & All \\
\hline$C_{W W}(\times 100)$ & $50.5 \pm 1.6$ & $40.3 \pm 1.7$ & $68.7 \pm 2.1$ & $52.0 \pm 1.7$ \\
Trigger efficiency & $0.3 \%$ & $0.1 \%$ & $0.6 \%$ & $0.4 \%$ \\
Lepton efficiency & $1.4 \%$ & $2.9 \%$ & $0.7 \%$ & $1.3 \%$ \\
Lepton $p_{\mathrm{T}}$ scale and resolution & $0.6 \%$ & $0.9 \%$ & $0.8 \%$ & $0.5 \%$ \\
Jet energy scale and resolution & & & & \\
Jet veto & $0.7 \%$ & $1.2 \%$ & $1.0 \%$ & $1.0 \%$ \\
Other cuts & $0.5 \%$ & $0.6 \%$ & $0.5 \%$ & $0.5 \%$ \\
$p_{\mathrm{T}}^{\text {inv } \text { modelling }}$ & $0.4 \%$ & $0.5 \%$ & $0.2 \%$ & $0.2 \%$ \\
PDFs, $\mu_{\mathrm{R}}$ and $\mu_{\mathrm{F}}$ scales & & & & \\
Acceptance & $0.3 \%$ & $0.7 \%$ & $0.7 \%$ & $0.3 \%$ \\
Jet veto correction factor & $2.6 \%$ & $2.6 \%$ & $2.6 \%$ & $2.6 \%$ \\
\hline$A_{W W}(\times 100)$ & $15.9 \pm 0.9$ & $7.5 \pm 0.4$ & $8.1 \pm 0.5$ & $11.9 \pm 0.7$ \\
PDFs, $\mu_{\mathrm{R}}$ and $\mu_{\mathrm{F}}$ scales & & & & \\
Jet veto & $5.6 \%$ & $5.6 \%$ & $5.6 \%$ & $5.6 \%$ \\
Other cuts & $1.1 \%$ & $1.0 \%$ & $1.0 \%$ & $1.0 \%$ \\
\hline$C_{W W} \cdot A_{W W}(\times 100)$ & $8.03 \pm 0.33$ & $3.02 \pm 0.15$ & $5.56 \pm 0.22$ & $6.19 \pm 0.25$ \\
PDFs, $\mu_{\mathrm{R}}$ and $\mu_{\mathrm{F}}$ scales & & & & $3.5 \%$ \\
Jet veto & $3.5 \%$ & $3.5 \%$ & $3.5 \%$ & \\
\hline
\end{tabular}

Table 6.5: The signal acceptances $C_{W W}, A_{W W}$ and $C_{W W} \cdot A_{W W}$. A breakdown of the relative uncertainties from different sources is also shown. The contribution to $\Delta\left(C_{W W} \cdot A_{W W}\right)$ of the theoretical uncertainty in the jet veto is given, in order to explicitly exhibit the cancellations between $\Delta C_{W W}$ and $\Delta A_{W W}$. 


\begin{tabular}{cc}
\hline Process & MC generator \\
\hline$t \bar{t}$ & MC@NLO+HERWIG \\
$t W, t b, t b q$ & ACERMC+Pythia 6 \\
$W+$ jet $, Z / \gamma^{*}, W \gamma$ & ALPGEN+HeRwiG \\
$W \gamma^{*}$ & MADGRAPH+PYTHIA 6 \\
$W Z, Z Z$ & HeRWiG \\
\hline
\end{tabular}

Table 6.6: $\mathrm{MC}$ generators used to model backgrounds to the $W W$ measurement.

\section{$\mathrm{W}+$ jet and dijet}

The $W+$ jet background comprises events where a jet is misidentified as a lepton. The rate of misidentification, or "fake rate", is poorly modelled by MC, and so a data-driven fake factor method is used. The dijet background, where two jets fake leptons, is very small and is simultaneously estimated by this method.

A $W+$ jet anti-ID region (AR) is defined similarly to the signal region (SR), but where one of the leptons is replaced with an anti-identified lepton, $\ell$. The $\&$ objects are ensured to be highly contaminated with jets by loosening the selection criteria and vetoing leptons passing the full identification. Anti-ID electrons, $\phi$, have looser calorimeter isolation criteria and do not have to pass the tight identification criteria. Anti-ID muons, $\mu$, have looser calorimeter isolation criteria and the tracker isolation removed completely. The predicted $W+$ jet background is the measured number of events in the AR, scaled by a fake factor $f_{\ell}$ :

$$
\begin{aligned}
N_{W+\text { jet }}^{\text {pred,SR }} & =f_{e} \cdot N_{e \phi}^{\text {data,AR }} \\
N_{W+\text { jet }}^{\text {pred,SR }} & =f_{\mu} \cdot N_{\mu \mu}^{\text {data,AR }} \\
N_{W+\text { jet }}^{\text {pred,en }} & =f_{e} \cdot N_{\mu \phi}^{\text {data,AR }}+f_{\mu} \cdot N_{\mu e}^{\text {data,AR }} .
\end{aligned}
$$

The fake factor $f_{\ell}$ is defined as the ratio of efficiencies for jets passing the lepton ID criteria to jets passing the anti-ID criteria. Each fake factor is measured from dijet events as a function of $p_{\mathrm{T}}$ and $\eta$ :

$$
f_{\ell}=\frac{N_{\ell}^{\text {data }}}{N_{\ell}^{\text {data }}} .
$$


To avoid bias, the dijet events were selected with a very loose (but prescaled) trigger, without identification or isolation criteria applied. A $Z$ veto $\left(\left|m_{\ell \ell}-m_{Z}\right|>15 \mathrm{GeV}\right)$ and a $W$ veto $\left(m_{\mathrm{T}}>30 \mathrm{GeV}\right)$ reject most of the prompt leptons, and residual electroweak contamination is subtracted using MC.

The dominant source of uncertainty stems from the fact that the fake factor is derived from dijet events but applied to $W+$ jet events. This process dependence is estimated with MC. It would be preferable to measure the fake factor from $Z+$ jet events, though this was statistically limited in the $\sqrt{s}=7 \mathrm{TeV}$ dataset. This point shall be revisited in Section 7.1 when considering the $W+$ jet background to the $H \rightarrow W W$ search.

\section{Top}

The top background is suppressed by the jet veto, and its yield is estimated by a data-driven template method. This constrains the top $N_{\text {jets }}$ distribution within a $b$-tagged control region (CR), subtracting contamination via a data-driven template fit. Then the 0-jet yield is extrapolated from the CR to the SR using MC.

In detail, an extended signal region (ESR) is defined by removing the jet veto and the $p_{\mathrm{T}, \ell \ell}$ cut. The top CR is a subset of the ESR, requiring at least one $b$-tagged jet with $p_{\mathrm{T}}>20 \mathrm{GeV}$. The top $N_{\text {jets }}$ distribution, $\mathcal{T}$, in the ESR is predicted by measuring $\mathcal{T}$ in the $\mathrm{CR}$ and extrapolating using $\mathrm{MC}$ :

$$
\mathcal{T}_{\text {top }}^{\text {pred,ESR }}=\frac{\mathcal{T}_{\text {top }}^{\mathrm{MC}, \mathrm{ESR}}}{\mathcal{T}_{\text {top }}^{\mathrm{MC}, \mathrm{CR}}}\left(\mathcal{T}^{\text {data,CR }}-K_{\text {non-top }}^{\mathrm{fit}} \cdot \mathcal{T}_{\text {non-top }}^{\mathrm{MC}, \mathrm{CR}}\right)
$$

The non-top contamination in the $\mathrm{CR}$ is described by $\mathrm{MC}$ and scaled by a datadriven normalisation factor $K_{\text {non-top }}^{\text {fit }}$ This $K_{\text {non-top }}^{\text {fit }}$ is assumed to be the same in the CR and the ESR, and is constrained by an $N_{\text {jets }}$ fit in the ESR, i.e.

$$
\mathcal{T}^{\text {data,ESR }}=\mathcal{T}_{\text {top }}^{\text {pred,ESR }}+K_{\text {non-top }}^{\text {fit }} \cdot \mathcal{T}_{\text {non-top }}^{\mathrm{MCESR}}
$$

Note that this fit depends upon $K_{\text {non-top }}^{\text {fit }}$ twice: directly and via $\mathcal{T}_{\text {top }}^{\text {pred,ESR }}$. The fit yields $K_{\text {non-top }}^{\text {fit }}=1.07 \pm 0.03$. Finally, the 0 -jet bin of $\mathcal{T}_{\text {top }}^{\text {pred,ESR }}$ is extrapolated to the SR using MC.

The small number of 0 -jet events in the top CR leads to a large statistical uncertainty in this background. The systematic uncertainty is dominated by uncertainties in the (mis)tag efficiency of the $b$-tagging algorithm. 


\section{$\mathrm{Z} / \gamma^{*}$}

The $Z / \gamma^{*}$ background is suppressed by the $Z$ mass veto and the $E_{\mathrm{T}, \text { rel }}^{\text {miss }}$ and $p_{\mathrm{T}, \ell \ell}$ cuts, though remains a significant background to the $e e$ and $\mu \mu$ channels. Since MC might mismodel the $E_{\mathrm{T} \text {,rel }}^{\text {miss }}$ distribution in $Z / \gamma^{*}$ events, this background is normalised to data in a control region $(\mathrm{CR})$ that includes the $E_{\mathrm{T}, \text { rel }}^{\text {miss }}$ cut.

The $Z / \gamma^{*} \mathrm{CR}$ is defined similarly to the signal region (SR), with the $p_{\mathrm{T}, \ell \ell}$ cut inverted. The number of events in the $\mathrm{CR}$ is measured, the non- $Z / \gamma^{*}$ contamination is subtracted via MC, and the result is extrapolated to the SR using MC:

$$
N_{Z / \gamma^{*}}^{\mathrm{pred}, \mathrm{SR}}=\frac{N_{Z / \gamma^{*}}^{\mathrm{MC}}}{N_{Z / \gamma^{*}}^{\mathrm{MC} C \mathrm{CR}}}\left(N^{\mathrm{data}, \mathrm{CR}}-N_{\mathrm{non}-Z / \gamma^{*}}^{\mathrm{MC}, \mathrm{CR}}\right) .
$$

The dominant uncertainties are due to the small number of events in the CR and the energy scale of the soft terms in the $E_{\mathrm{T}, \mathrm{rel}}^{\text {miss }}$ (see Section 4.2.7).

\section{Non-WW diboson}

The non- $W W$ diboson backgrounds $\left(W Z, W \gamma^{*}, Z Z, W \gamma\right)$ are estimated purely from MC simulation (see Table 6.6). The cross section of each process is calculated at NLO with MCFM [137]. The dominant uncertainties are theoretical uncertainties in the cross section and JES uncertainties in the jet veto.

\subsubsection{Experimental results}

The observed and expected $p_{\mathrm{T}, \ell}^{\text {lead }}$ and $\Delta \phi(\ell, \ell)$ distributions in the signal region are displayed in Figure 6.2, and the observed number of events passing the event selection is shown for each channel in Table 6.7. The expected number of events is also shown, with the signal and background contributions estimated as described in Sections 6.1.4 and 6.1.5, respectively. Systematic uncertainties in the $Z / \gamma^{*}$ and non- $W W$ diboson backgrounds are considered correlated (since they are both dominated by uncertainties in the calorimeter energy scale), and those in the other backgrounds are considered uncorrelated. A comparison of data to expectation suggests that the measured cross section shall be higher than predicted.

The fiducial and total cross sections are calculated for each channel with (6.1) and (6.2) respectively, using the values in Tables 6.5 and 6.7 and an integrated luminosity of $L=4.64 \pm 0.18 \mathrm{fb}^{-1}$. Systematic uncertainties in the signal acceptance and the total 

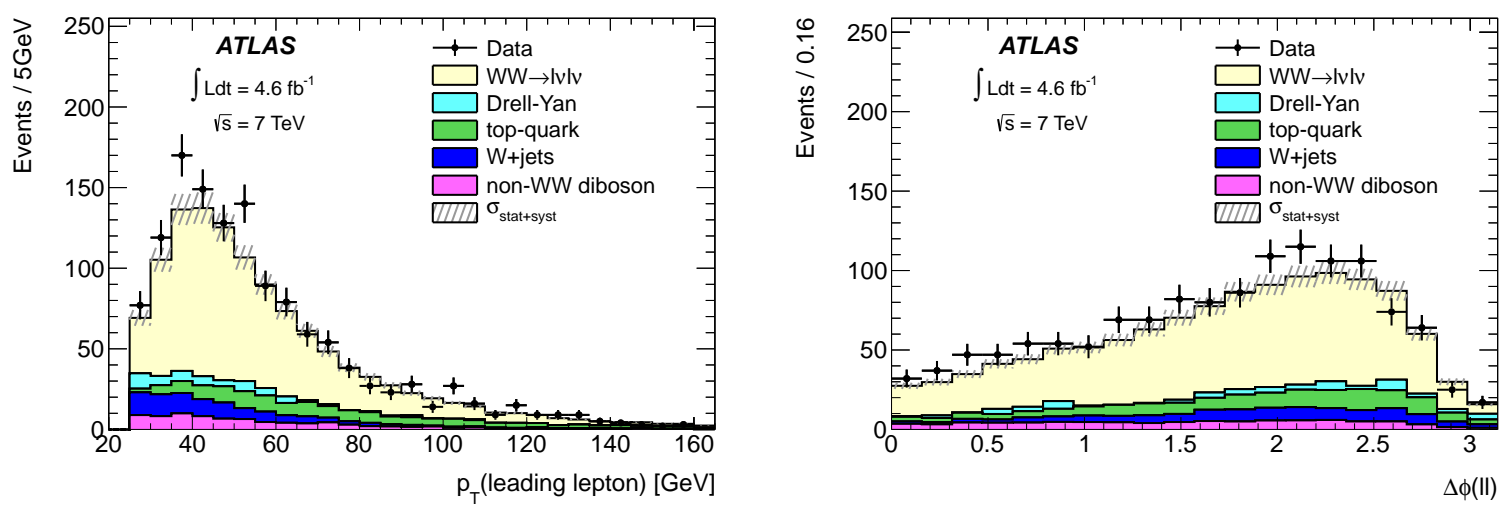

Figure 6.2: The $p_{\mathrm{T}, \ell}^{\text {lead }}($ left) and $\Delta \phi(\ell, \ell)$ (right) distributions for the selected $W W$ candidates, including $e \mu$, ee and $\mu \mu$ channel events.

\begin{tabular}{|c|c|c|c|c|}
\hline & $e \mu$ & $e e$ & $\mu \mu$ & All \\
\hline Observed & 821 & 174 & 330 & 1325 \\
\hline Expected & $744 \pm 24 \pm 57$ & $169 \pm 12 \pm 16$ & $280 \pm 16 \pm 20$ & $1192 \pm 31 \pm 87$ \\
\hline$W W$ & $538 \pm 3 \pm 45$ & $100 \pm 2 \pm 9$ & $186 \pm 2 \pm 15$ & $824 \pm 4 \pm 69$ \\
\hline Background & $206 \pm 24 \pm 35$ & $68 \pm 12 \pm 13$ & $94 \pm 15 \pm 13$ & $369 \pm 31 \pm 53$ \\
\hline Top & $87 \pm 23 \pm 13$ & $22 \pm 12 \pm 3$ & $32 \pm 14 \pm 5$ & $141 \pm 30 \pm 22$ \\
\hline$W+$ jet & $70 \pm 2 \pm 31$ & $21 \pm 1 \pm 11$ & $7 \pm 1 \pm 3$ & $98 \pm 2 \pm 43$ \\
\hline$Z / \gamma^{*}$ & $5 \pm 2 \pm 1$ & $12 \pm 3 \pm 3$ & $34 \pm 6 \pm 10$ & $51 \pm 7 \pm 12$ \\
\hline Diboson & $44 \pm 2 \pm 6$ & $13 \pm 1 \pm 2$ & $21 \pm 1 \pm 2$ & $78 \pm 2 \pm 10$ \\
\hline
\end{tabular}

Table 6.7: The number of events observed and expected in the $4.6 \mathrm{fb}^{-1}$ dataset in each signal region. A breakdown of the expected signal and background contributions is also shown, with statistical and systematic uncertainties. The $W W$ signal is normalised to the NLO cross section of $44.7 \mathrm{pb}$. 
background estimation are assumed uncorrelated, and propagate to the cross sections via

$$
\begin{aligned}
& \left(\frac{\Delta \sigma_{W W}^{\mathrm{fid}}}{\sigma_{W W}^{\mathrm{fid}}}\right)_{\mathrm{syst}}^{2}=\left(\frac{\Delta C_{W W}}{C_{W W}}\right)^{2}+\left(\frac{\Delta N_{\mathrm{bkg}}}{N_{\mathrm{obs}}-N_{\mathrm{bkg}}}\right)^{2}+\left(\frac{\Delta L}{L}\right)^{2} \\
& \left(\frac{\Delta \sigma_{W W}}{\sigma_{W W}}\right)_{\mathrm{syst}}^{2}=\left(\frac{\Delta\left(C_{W W} \cdot A_{W W}\right)}{C_{W W} \cdot A_{W W}}\right)^{2}+\left(\frac{\Delta N_{\mathrm{bkg}}}{N_{\mathrm{obs}}-N_{\mathrm{bkg}}}\right)^{2}+\left(\frac{\Delta L}{L}\right)^{2} .
\end{aligned}
$$

The relative statistical uncertainty in each channel is simply $\sqrt{N_{\mathrm{obs}}} /\left(N_{\mathrm{obs}}-N_{\mathrm{bkg}}\right)$.

The combined total cross section from the three channels is obtained by maximising the likelihood function of a Poisson process

$$
\begin{aligned}
\mathcal{L}\left(\sigma_{W W} \mid N_{\mathrm{obs}}^{e \mu}, N_{\mathrm{obs}}^{e e}, N_{\mathrm{obs}}^{\mu \mu}\right) & =\operatorname{Pois}\left(N_{\mathrm{obs}}^{e \mu} ; \sigma_{W W}\right) \cdot \operatorname{Pois}\left(N_{\mathrm{obs}}^{e e} ; \sigma_{W W}\right) \cdot \operatorname{Pois}\left(N_{\mathrm{obs}}^{\mu \mu} ; \sigma_{W W}\right) \\
& =\prod_{i=1}^{3} \frac{e^{-\left(N_{\mathrm{sig}}^{i}+N_{\mathrm{bkg}}^{i}\right)} \cdot\left(N_{\mathrm{sig}}^{i}+N_{\mathrm{bkg}}^{i}\right)^{N_{\mathrm{obs}}^{i}}}{N_{\mathrm{obs}}^{i} !}
\end{aligned}
$$

where $N_{\mathrm{sig}}^{i}=\sigma_{W W} \cdot C_{W W}^{i} \cdot A_{W W}^{i} \cdot \mathrm{BR}^{i} \cdot L$ and the product over $i$ corresponds to the three channels. The best fit $\hat{\sigma}_{W W}$ is that which maximises the likelihood, and the statistical uncertainty is obtained by finding where $\ln \mathcal{L}\left(\hat{\sigma}_{W W} \pm \Delta \sigma_{W W}\right)=\ln \mathcal{L}\left(\hat{\sigma}_{W W}\right)-\frac{1}{2}$.

The systematic uncertainty in the combined cross section is calculated with (6.12), again using the values in Tables 6.5 and 6.7. The top and non- $W W$ diboson systematic uncertainties are considered correlated between all three channels (since the $b$-tagging efficiency, JES and total diboson cross sections are independent of lepton flavour). The $W+$ jet and $Z / \gamma^{*}$ systematic uncertainties are treated as uncorrelated between the ee and $\mu \mu$ channels, though the $e e+\mu \mu$ combination is considered correlated with the $e \mu$ channel (since the $f_{e}$ and $f_{\mu}$ fake factors are determined independently, and the $Z / \gamma^{*} \rightarrow \ell \ell$ uncertainty is related to lepton energy mismeasurement).

The measured cross sections are shown in Table 6.8. The combined total cross section is $51.9 \pm 2.0$ (stat) \pm 3.9 (syst) \pm 2.0 (lumi) pb, which is slightly higher than the theoretical prediction of $44.7 \pm 2.0 \mathrm{pb}$. However, the discrepancy is not significant. If contributions from $H \rightarrow W W$ and VBF $W W$ production are included as backgrounds, the measured cross section reduces to $50.5 \pm 2.0$ (stat) \pm 3.9 (syst) \pm 2.0 (lumi) pb. 


\begin{tabular}{ccccc}
\hline & \multicolumn{2}{c}{ Fiducial cross section (fb) } & \multicolumn{2}{c}{ Total cross section (pb) } \\
& Measured & Predicted & Measured & Predicted \\
\hline$e \mu$ & $262.3 \pm 12.3 \pm 20.7 \pm 10.2$ & $231.4 \pm 15.7$ & $51.1 \pm 2.4 \pm 4.2 \pm 2.0$ & $44.7 \pm 2.0$ \\
$e e$ & $56.4 \pm 6.8 \pm 9.8 \pm 2.2$ & $54.6 \pm 3.7$ & $46.9 \pm 5.7 \pm 8.2 \pm 1.8$ & $44.7 \pm 2.0$ \\
$\mu \mu$ & $73.9 \pm 5.9 \pm 6.9 \pm 2.9$ & $58.9 \pm 4.0$ & $56.7 \pm 4.5 \pm 5.5 \pm 2.2$ & $44.7 \pm 2.0$ \\
\hline All & & & $51.9 \pm 2.0 \pm 3.9 \pm 2.0$ & $44.7 \pm 2.0$ \\
\hline
\end{tabular}

Table 6.8: Measured fiducial and total $W W$ cross sections extracted from each signal region. Theoretical predictions are shown for comparison. The uncertainties in measured quantities are statistical, systematic and luminosity, respectively.

\subsection{Background estimation for $\mathrm{H} \rightarrow \mathrm{WW}$ search}

Non-resonant $W W$ production is an irreducible background to the $H \rightarrow W W$ search, and is the dominant background contribution following the event selection described in Chapter 4. Thus, it is critical that it is accurately estimated.

The $W W$ process is modelled at NLO by PowhegBox+Pythia 6. It is necessary to use PowhegBox since MC@NLO does not feature "singly resonant diagrams", where the mass of the dilepton + dineutrino system is that of a single $Z$ boson. This occurs in $Z / \gamma^{*} \rightarrow \ell \ell$ events where a lepton radiates a $W$ boson, and the final state is indistinguishable from the $W W$ process. Since the $H \rightarrow W W$ search is sensitive to offshell $W$ bosons, it is important to include these diagrams. The PowhegBox+Pythia 6 event generator is used as it is found to better describe the experimental data in many exclusive observables, when compared to PowhegBox+Pythia 8. This is likely to be related to the PowhegBox+Pythia 8 matching issues mentioned in Section 2.2.6. Also, the ATLAS underlying event AUET2B tune [138] was found to be overtuned to dijet data and consequently its description of electroweak processes suffered. For this reason, the updated Perugia 2011C Pyтhia 6 tune [139] was used. NNLO $g g \rightarrow W W$ diagrams are modelled by GG2Ww+HERWIG [135].

To estimate the $W W$ contribution to the signal region (SR), a data-driven technique is employed whereby MC is used to extrapolate from a high-purity control region (CR) 


\begin{tabular}{|c|c|}
\hline \multicolumn{2}{|c|}{$e \mu / \mu e$} \\
\hline \multicolumn{2}{|c|}{$\begin{array}{c}p_{\mathrm{T}, \ell}^{\text {lead }}>22 \text { and } p_{\mathrm{T}, \ell}^{\text {sublead }}>15 \\
p_{\mathrm{T}}^{\text {miss,corr }}>20\end{array}$} \\
\hline 0 -jet bin & 1-jet bin \\
\hline$p_{\mathrm{T}, \ell \ell}>30$ & $\left|m_{\tau \tau}-m_{Z}\right|>25$ \\
\hline$\Delta \phi\left(\ell \ell, p_{\mathrm{T}}^{\mathrm{inv}}\right)>\pi / 2$ & $\max \left(m_{\mathrm{T}, \ell}\right)>50$ \\
\hline- & $N_{b \text {-jets }}=0$ \\
\hline $55<m_{\ell \ell}<110$ & $m_{\ell \ell}>80$ \\
\hline$\Delta \phi(\ell, \ell)<2.6$ & - \\
\hline
\end{tabular}

Table 6.9: Event selection criteria of the $W W$ control regions (not used in the $\geq 2$-jet bin). Cuts on energy, momentum and mass are given in $\mathrm{GeV}$, and angular cuts are given in radians. The relevant observables are described in Chapter 4.

to the SR:

$$
\begin{aligned}
N_{W W}^{\mathrm{pred}, \mathrm{SR}} & =\alpha_{W W} \cdot\left(N^{\mathrm{data}, \mathrm{CR}}-N_{\mathrm{non}-W W}^{\mathrm{pred}, \mathrm{CR}}\right) \\
\alpha_{W W} & =N_{W W}^{\mathrm{MC}, \mathrm{SR}} / N_{W W}^{\mathrm{MC}, \mathrm{CR}}
\end{aligned}
$$

where $N_{\mathrm{non}-W W}^{\mathrm{pred}, C \mathrm{R}}$ is determined by dedicated methods (see Chapter 7 ). The CR is chosen to reduce $N_{\text {non- } W W}^{\text {pred }}$ to avoid bias, whilst maintaining a large $N^{\text {data,CR }}$ to minimise the statistical uncertainty in $N_{W W}^{\mathrm{pred}, \mathrm{SR}}$. The MC-based extrapolation $\alpha_{W W}$ introduces experimental and theoretical uncertainties to $N_{W W}^{\mathrm{pred}, \mathrm{SR}}$.

As explained in Section 4.3.4, the resonant $H \rightarrow W W$ and non-resonant $W W$ processes are distinguished by the scalar nature of the Higgs boson, which affects the decay topology of the $W$ bosons. This causes $H \rightarrow W W$ events to have a small dilepton opening angle, and consequently low $m_{\ell \ell}$ and $\Delta \phi(\ell, \ell)$. It is therefore intuitive to define the $W W \mathrm{CR}$ at high $m_{\ell \ell}$, with a relaxed $\Delta \phi(\ell, \ell)$ requirement.

Since jet binning can introduce large uncertainties, a $W W \mathrm{CR}$ is defined for each of the 0 -jet and 1-jet bins separately (see Table 6.9). Unfortunately, it is not possible to define a $W W \mathrm{CR}$ in the $\geq 2$-jet bin with sufficient purity owing to the large top background. Thus, the $W W$ background estimation in the $\geq 2$-jet bin is MC-based (see Section 6.2.3). 

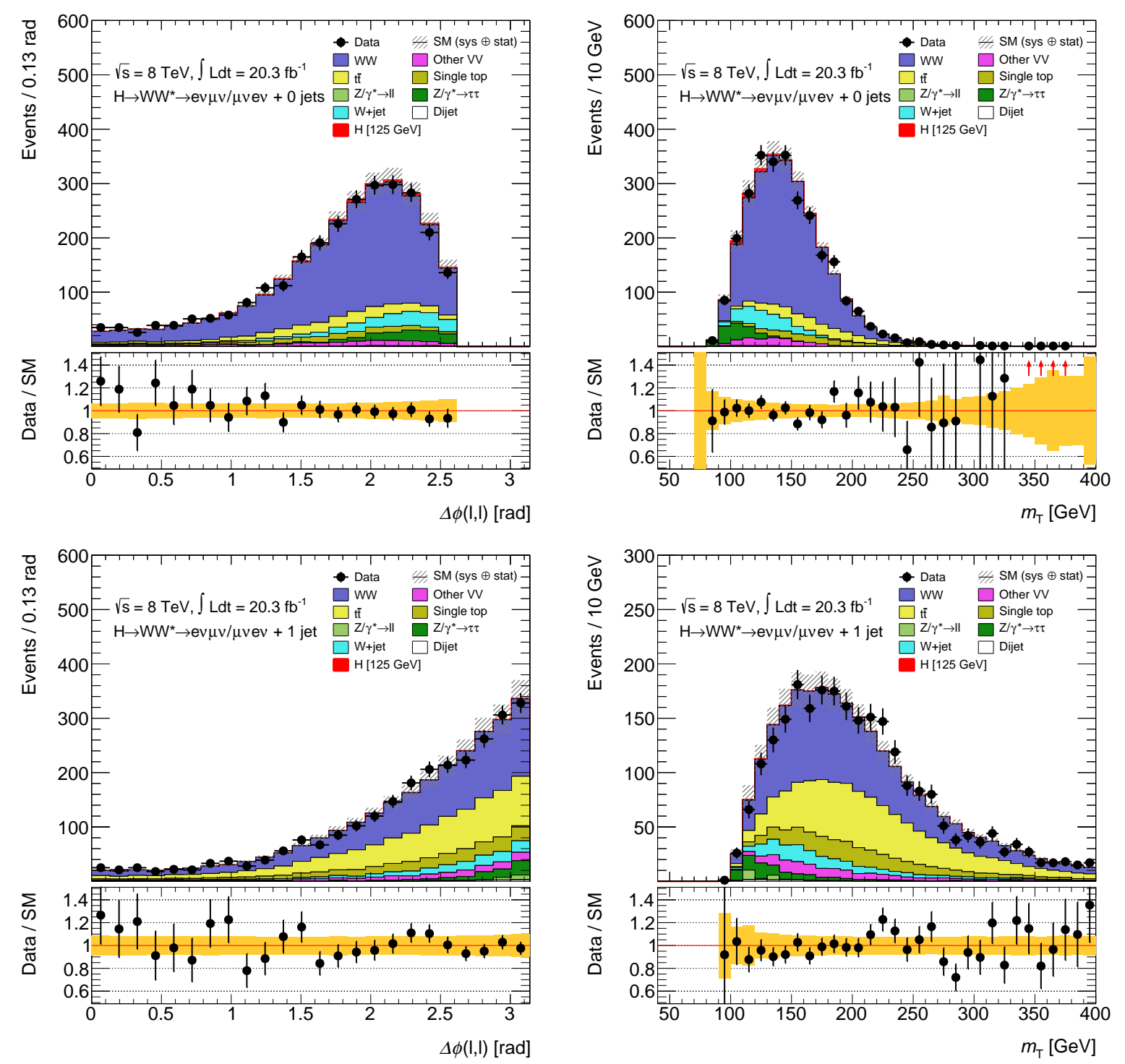

Figure 6.3: The $\Delta \phi(\ell, \ell)$ (left) and $m_{\mathrm{T}}$ (right) distributions in the $W W$ control regions of the 0-jet (top) and 1-jet (bottom) bins. Normalisation factors are applied.

Since the $e e / \mu \mu$ channels are dominated by $Z / \gamma^{*} \rightarrow \ell \ell$, they are extrapolated from $e \mu / \mu e$ CRs. The selection criteria follow those of the SRs (see Table 4.5), with different $m_{\ell \ell}$ and $\Delta \phi(\ell, \ell)$ cuts. However, the $p_{\mathrm{T}, \ell}^{\text {sublead }}$ threshold is raised from $10 \mathrm{GeV}$ to $15 \mathrm{GeV}$ in order to reduce contamination from the $W+$ jet background. A validation region (VR) is also defined in the 0 -jet bin with $m_{\ell \ell}>110 \mathrm{GeV}$, to test the extrapolation from the CR.

Figure 6.3 exhibits the excellent description of the shapes of distributions in the CRs, following application of the normalisation factors. 


\subsubsection{Theoretical uncertainties in $\alpha_{w w}$}

Theoretical uncertainties in the extrapolation parameters $\alpha_{W W}$ are evaluated at hadronlevel (i.e. before detector simulation) by changing some aspect of the MC modelling and measuring the effect upon each $\alpha_{W W}$. This is done using NLO $W W$ MC. Uncertainties due to $g g \rightarrow W W$ diagrams are $\sim 7 \%$, but have small impact on the uncertainty in the measured signal since these diagrams only contribute $\sim 5 \%(\sim 7 \%)$ of the $W W$ background in the 0-jet (1-jet) bin SRs.

The hadron-level event selection criteria used to evaluate these uncertainties are similar to the detector-level criteria of the SRs (see Table 4.5) and CRs (see Table 6.9), except the $b$-jet veto and $f_{\text {recoil }}$ cuts are omitted. Hadron-level object definitions are the same as those used for the $W W$ measurement (see Section 6.1.3), with updated $p_{\mathrm{T}}$ requirements.

Four sources of theoretical uncertainty are considered:

- higher order corrections (QCD and EW),

- PDFs,

- parton shower, hadronisation and underlying event models,

- NLO-PS matching scheme.

Uncertainties due to QCD higher order corrections are evaluated via independent variation of renormalisation and factorisation scales in the range $\mu_{0} / 2 \leq \mu_{\mathrm{R}}, \mu_{\mathrm{F}} \leq 2 \mu_{0}$, where $\mu_{0}=m_{\ell \nu \ell^{\prime} \nu^{\prime}}$, whilst observing the constraint $1 / 2 \leq \mu_{\mathrm{R}} / \mu_{\mathrm{F}} \leq 2$. These are evaluated with AMC@NLO and validated with MCFM. The largest deviation is used as the uncertainty.

Uncertainties due to EW higher order corrections are evaluated by reweighting PowheGBox events (based upon the kinematics of the initial state and diboson system) to include NLO EW corrections. Such corrections are derived in reference [140].

Uncertainties due to PDFs are evaluated in two ways, which are added in quadrature. Predictions with the MSTW 2008 [58] and NNPDF 2.3 [63] PDF sets are compared to those with the CT10 [62] PDF sets, and the maximum deviation is found. Also, the set of PDF eigenvectors corresponding to $90 \% \mathrm{CL}$ of the CT10 fit were used to evaluate an uncertainty, which was then rescaled to 68\% CL. These are evaluated using AMC@NLO.

Uncertainties due to the PS, hadronisation and UE models are evaluated by comparing PowhegBox showered by Pythia 6 (nominal) and by Herwig. Uncertainties due to 
the NLO-PS matching scheme are evaluated by comparing PowheGBox+HeRwig to AMC@NLO+HERWIG.

The $\alpha_{W W}$ theoretical uncertainties for each SR are shown in Table 6.10 (experimental uncertainties are discussed in Chapter 8). Although small, they have a large effect in the final fit because the $W W$ background yield is much larger than the signal yield in the SR. Extrapolation uncertainties for the VR are also shown. The observed number of events in the $W W$ VR is $10 \%$ higher than expected, corresponding to a $0.65 \sigma$ deviation when statistical and systematic uncertainties are considered. This agreement supports the extrapolation uncertainties assigned to the SRs.

\subsection{2 $\mathrm{m}_{\mathrm{T}}$ shape modelling}

Theoretical uncertainties in the shape of the $m_{\mathrm{T}}$ distribution are also investigated, as they affect the fit. Uncertainties due to scale, PS/UE and NLO-PS choices are considered within each signal region separately, using the methods described above.

Each uncertainty is parametrised by fitting the ratio of the $m_{\mathrm{T}}$ shapes, and then symmetrising the fit to produce "up" and "down" variations. The $m_{\mathrm{T}}$ distributions are normalised to unit integral in order to probe only shape uncertainties. A linear fit is used in the central $m_{\mathrm{T}}$ region, and a constant is used in the low- $m_{\mathrm{T}}$ and high- $m_{\mathrm{T}}$ tails of the distribution where statistical fluctuations dominate.

These fits allow the hadron-level $m_{\mathrm{T}}$ distribution of the $W W$ background to be reweighted to the "up" and "down" variations. In this way, the $m_{\mathrm{T}}$ shape uncertainty is treated as a nuisance parameter in the $H \rightarrow W W$ fitting procedure (see Chapter 8). The uncertainties for the split signal regions of the $e \mu / \mu e$ channels are displayed in Figures 6.4 and 6.5 for the 0 -jet and 1 -jet bins, respectively. 


\begin{tabular}{|c|c|c|c|c|c|c|c|c|}
\hline & \multirow{2}{*}{$\begin{array}{c}m_{\ell \ell} \\
(\mathrm{GeV})\end{array}$} & \multirow{2}{*}{$\begin{array}{c}p_{\mathrm{T}, \ell}^{\text {sublead }} \\
(\mathrm{GeV})\end{array}$} & \multicolumn{2}{|c|}{ Scale } & \multicolumn{2}{|c|}{ PDF } & \multirow{2}{*}{$\mathrm{PS} / \mathrm{UE}$} & \multirow{2}{*}{ NLO-PS } \\
\hline & & & $\mathrm{QCD}$ & EW & collab. & $68 \% \mathrm{CL}$ & & \\
\hline \multicolumn{9}{|c|}{$e e / \mu \mu$ channels } \\
\hline 0 -jet & $12-55$ & $>10$ & $0.8 \%$ & $+0.1 \%$ & $0.5 \%$ & $1.0 \%$ & $-1.2 \%$ & $+2.4 \%$ \\
\hline 1-jet & $12-55$ & $>10$ & $0.8 \%$ & $-2.1 \%$ & $0.5 \%$ & $0.7 \%$ & $-2.3 \%$ & $+3.8 \%$ \\
\hline \multicolumn{9}{|c|}{$e \mu / \mu e$ channels } \\
\hline \multirow{6}{*}{0 -jet } & & $10-15$ & $0.7 \%$ & $+1.2 \%$ & $0.9 \%$ & $0.2 \%$ & $+2.2 \%$ & $+0.4 \%$ \\
\hline & $10-30$ & $15-20$ & $1.2 \%$ & $+0.7 \%$ & $0.8 \%$ & $0.2 \%$ & $+1.7 \%$ & $+0.9 \%$ \\
\hline & & $>20$ & $0.7 \%$ & $-0.3 \%$ & $0.5 \%$ & $0.3 \%$ & $-1.9 \%$ & $+3.1 \%$ \\
\hline & & $10-15$ & $0.7 \%$ & $+0.8 \%$ & $0.8 \%$ & $0.1 \%$ & $+1.5 \%$ & $+0.5 \%$ \\
\hline & $30-55$ & $15-20$ & $0.8 \%$ & $+0.5 \%$ & $0.7 \%$ & $0.2 \%$ & $+1.0 \%$ & $+1.0 \%$ \\
\hline & & $>20$ & $0.8 \%$ & $-0.4 \%$ & $0.4 \%$ & $0.5 \%$ & $-2.4 \%$ & $+3.9 \%$ \\
\hline \multirow{6}{*}{ 1-jet } & & $10-15$ & $3.1 \%$ & $-0.9 \%$ & $0.5 \%$ & $0.1 \%$ & $-2.4 \%$ & $-3.4 \%$ \\
\hline & $10-30$ & $15-20$ & $1.6 \%$ & $-1.5 \%$ & $0.5 \%$ & $0.1 \%$ & $-3.0 \%$ & $+0.7 \%$ \\
\hline & & $>20$ & $1.0 \%$ & $-2.8 \%$ & $0.6 \%$ & $0.2 \%$ & $-3.6 \%$ & $+5.3 \%$ \\
\hline & & $10-15$ & $3.2 \%$ & $-0.9 \%$ & $0.5 \%$ & $0.1 \%$ & $-2.0 \%$ & $+1.9 \%$ \\
\hline & $30-55$ & $15-20$ & $1.5 \%$ & $-1.6 \%$ & $0.4 \%$ & $0.1 \%$ & $-3.0 \%$ & $+2.4 \%$ \\
\hline & & $>20$ & $1.3 \%$ & $-2.7 \%$ & $0.5 \%$ & $0.4 \%$ & $-3.1 \%$ & $+5.6 \%$ \\
\hline \multicolumn{9}{|c|}{ Validation region $(e \mu / \mu e$ channels) } \\
\hline 0 -jet & $>110$ & $>15$ & $0.6 \%$ & $+1.6 \%$ & $0.6 \%$ & $2.0 \%$ & $+4.3 \%$ & $-5.1 \%$ \\
\hline
\end{tabular}

Table 6.10: Theoretical uncertainties in the $W W$ extrapolation parameter $\alpha_{W W}$ for each signal region used in the fitting procedure, and also for the validation region. 


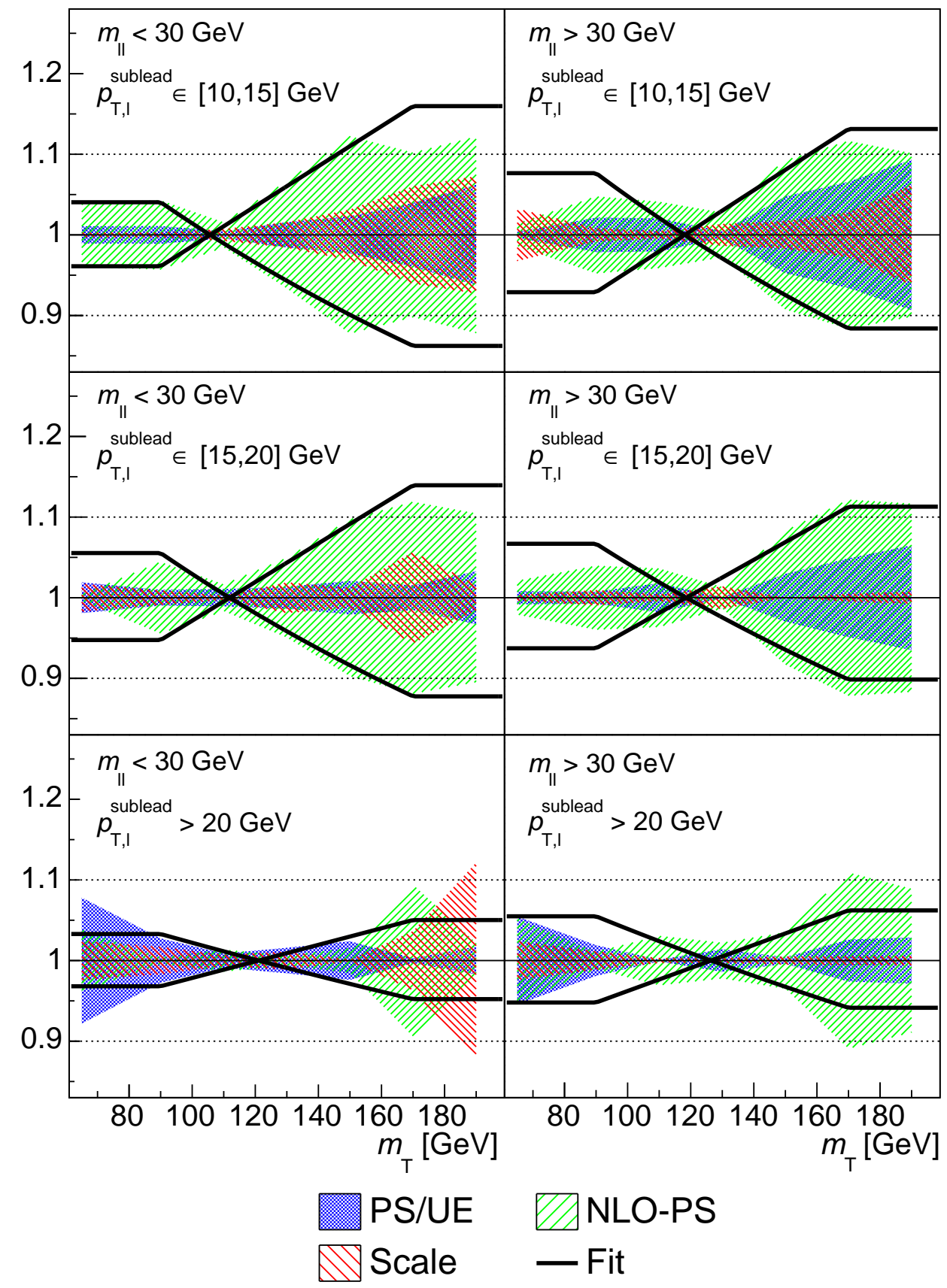

Figure 6.4: $W W m_{\mathrm{T}}$ shape systematic uncertainties in each 0-jet signal region for the $e \mu / \mu e$ channels. The fit shown is the sum in quadrature of the three individual fits, and successfully envelopes the uncertainty sources. 


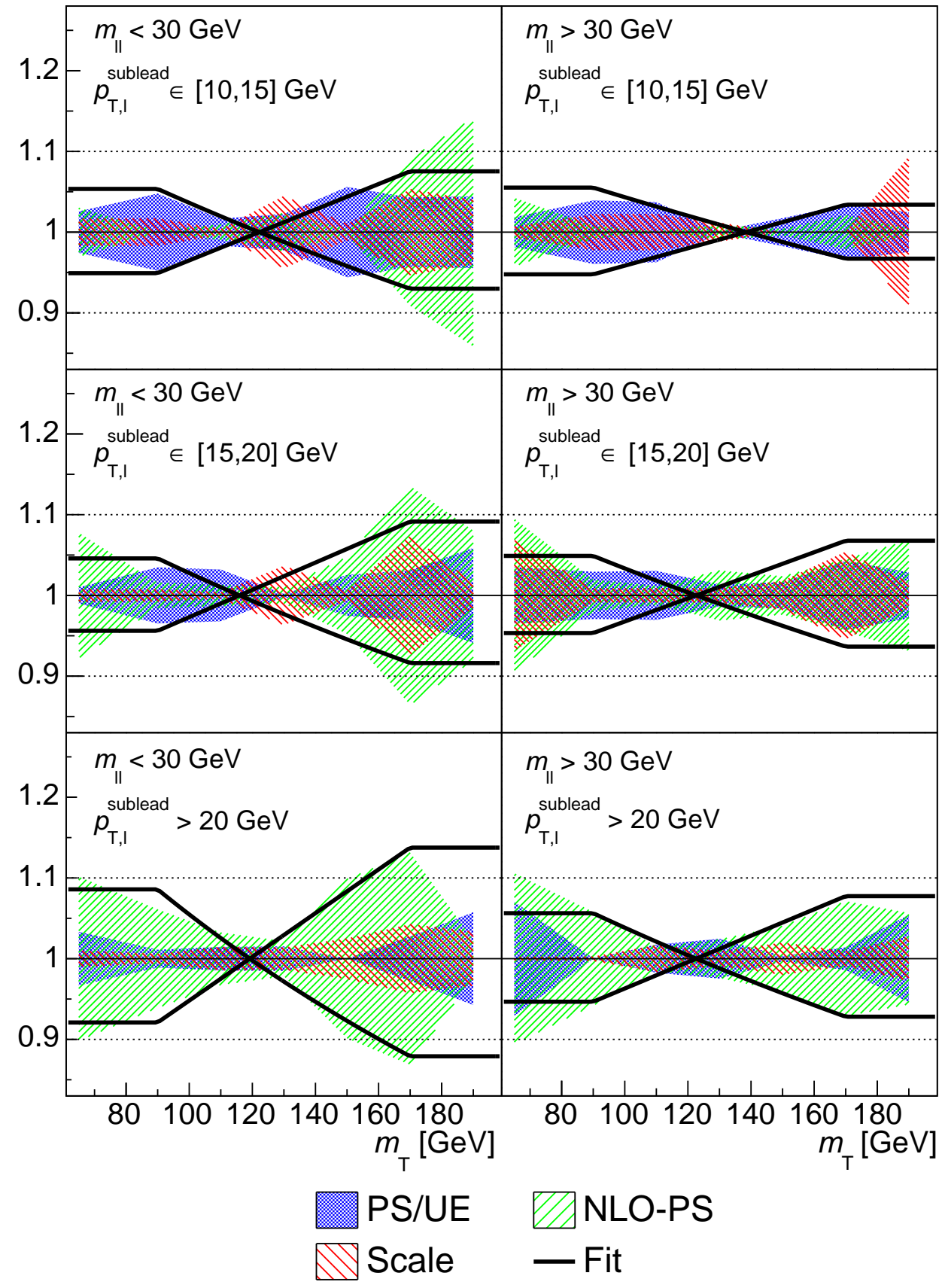

Figure 6.5: $W W m_{\mathrm{T}}$ shape systematic uncertainties in each 1-jet signal region for the $e \mu / \mu e$ channels. The fit shown is the sum in quadrature of the three individual fits, and successfully envelopes the uncertainty sources. 


\subsubsection{WW background in the $\geq 2$-jet bin}

PowhegBox relies upon the parton shower to produce the second emission; consequently events with two or more hard jets are likely to be poorly modelled. For this reason, SHERPA is used to describe the $W W$ background in the $\geq 2$-jet bin, including up to three partons in the matrix element using LO ME-PS merging (see Section 2.2.3).

Two kinds of diagrams can be identified for the $W W+2$ jets process: EW production (zero QCD vertices at $\mathrm{LO}$ ) and QCD production (two QCD vertices at $\mathrm{LO}$ ), where VBF $W W$ production is included in the former. The two production types are simulated separately by SHERPA, and this causes their interference to be neglected. Interference with VBF Higgs boson production is also neglected. The effect of each interference is investigated and treated as a systematic uncertainty.

Uncertainties due to the choice of $\mu_{\mathrm{R}}$ and $\mu_{\mathrm{F}}$ scale are considered, and also a modelling uncertainty is evaluated by comparing the SHERPA yield to that of MADGRAPH+PYTHIA 6. The uncertainty in QCD $W W+2$ jets production is $\sim 20 \%$, and the uncertainty in $\mathrm{EW} W W+2$ jets production is $\sim 10 \%$. 


\section{Chapter 7}

\section{Other backgrounds}

In addition to the $W W$ background, several other processes contribute significant background. As they are often difficult to model accurately in the $H \rightarrow W W$ phase space, they are estimated by sophisticated data-driven methods. Even so, there is usually some underlying dependence upon MC, which is summarised in Table 7.1.

This chapter describes the estimation of non- $W W$ backgrounds: $W+$ jet and dijet in Section 7.1, non- $W W$ diboson in Section 7.2, top in Section 7.3, and $Z / \gamma^{*}$ in Section 7.4.

\begin{tabular}{|c|c|}
\hline Process & MC generator $(\geq 2$-jet bin $)$ \\
\hline$W W$ & PowhegBox+Pythia 6, GG2Ww+Herwig (Sherpa) \\
\hline top & PowhegBox+Pythia 6 , AcerMC+Pythia 6 \\
\hline$W+$ jet $, Z / \gamma^{*}, W \gamma$ & AlPGEN+HERWIG \\
\hline$W Z$ & POWHEGBOX+PYTHIA 8 \\
\hline$Z Z$ & PowhegBox+Pythia 8, GG2ZZ+HeRWIG \\
\hline$W \gamma^{*}, Z \gamma^{*}, Z \gamma$ & SHERPA \\
\hline
\end{tabular}

Table 7.1: MC generators used to model backgrounds to the $H \rightarrow W W$ search. These are used with the data-driven techniques described in the text. 


\section{$7.1 \mathrm{~W}+$ jet and dijet}

$W+$ jet events contribute to the background when a jet is misidentified as a lepton, and dijet events contribute when two jets are misidentified as leptons. This is due to leptonic decays of heavy flavour hadrons, hadronic showers mimicking the electromagnetic showers, or punchthrough into the muon spectrometer. Although the fake rates are very low, these backgrounds are significant because the $W+$ jet and dijet cross sections are many orders of magnitude larger than those of Higgs boson production. Since these fake rates are sensitive to effects that are difficult to accurately model (such as jet flavour composition, jet substructure and hadronic shower shapes), a data-driven fake factor method is used to estimate these backgrounds.

As these two backgrounds have large uncertainties, their suppression is critically important. The electron and muon selection criteria are chosen to be tighter at low $p_{\mathrm{T}}$ (see Section 4.2), in order to reduce the fake rates where these backgrounds are largest. The dijet background is additionally rejected by requiring significant $p_{\mathrm{T}}^{\text {inv }}$ and by the $\max \left(m_{\mathrm{T}, \ell}\right)>50 \mathrm{GeV}$ cut in the 1-jet bin of the $e \mu / \mu e$ channels.

\subsubsection{The fake factor method}

The fake factor method defines two new objects: anti-identified electrons $\phi$ and muons $\mu$, collectively known as anti-identified leptons $\&$. Loosened selection criteria ensure they are highly contaminated by jets, while a veto on identified leptons $\ell$ removes overlap between $\ell$ and $\ell$ objects (see Section 7.1.2). The fake factor $f_{\ell}$ is then defined as the ratio of efficiencies (i.e. fake rates) for jets passing the $\ell$ criteria to passing the $\ell$ criteria.

In the following discussion, a sample shall refer to a collection of events featuring a specific set of objects, e.g. the signal sample $\mathcal{N}_{\ell \ell, \text { os }}$ refers to $\ell \ell$ events with opposite-sign charges (the $H \rightarrow W W$ analysis uses three signal samples: $\ell \ell=e e+\mu \mu, e \mu$ and $\mu e$ ). A region shall refer to events whose objects satisfy a set of criteria (also known as cuts), e.g. the signal region refers to events passing the criteria in Table 4.5.

A dilepton sample $\mathcal{N}_{\ell \ell}$, a $W+$ jet control sample $\mathcal{N}_{\ell \ell}$ and a dijet control sample $\mathcal{N}_{\text {lq }}$ are defined, each with opposite-sign (OS) and same-sign (SS) charge variants. Note that $\mathcal{N}$ indicates a sample to which cuts may be applied, and an $\ell$ is treated as an $\ell$ in such cuts when appropriate. Each sample has contributions from $W+$ jet and dijet events, and 
events with prompt leptons from the hard scatter or photon conversions (labelled EW):

$$
\begin{aligned}
& \mathcal{N}_{\ell \ell, i}=\mathcal{N}_{\ell \ell, i}^{W+\text { jet }}+\mathcal{N}_{\ell \ell, i}^{\text {dijet }}+\mathcal{N}_{\ell \ell, i}^{\mathrm{EW}} \\
& \mathcal{N}_{\ell \ell, i}=\mathcal{N}_{\ell \ell, i}^{W+\text { jet }}+\mathcal{N}_{\ell \ell, i}^{\text {dijet }}+\mathcal{N}_{\ell \ell, i}^{\mathrm{EW}} \\
& \mathcal{N}_{\ell \ell, i}=\mathcal{N}_{\ell \ell, i}^{W+\text { jet }}+\mathcal{N}_{\ell \ell, i}^{\text {dijet }}+\mathcal{N}_{\ell \ell, i}^{\mathrm{EW}}
\end{aligned}
$$

where $i=\mathrm{OS}$, SS. $\mathcal{N}_{\ell \ell, i}$ is dominated by $\mathcal{N}_{\ell \ell, i}^{\mathrm{EW}}, \mathcal{N}_{\ell \ell, i}$ is dominated by $\mathcal{N}_{\ell \phi, i}^{W+\text { jet }}$ and $\mathcal{N}_{\ell \phi, i}$ is dominated by $\mathcal{N}_{\& \text { dijet }}^{\text {diet }}$.

The purpose of the fake factor method is to estimate the $\mathcal{N}_{\ell \ell, \text { OS }}^{W+\text { jet }}$ and $\mathcal{N}_{\ell \ell \text {,OS }}^{\text {dijet }}$ contributions to the $\mathcal{N}_{\ell \ell, \text { OS }}$ signal sample. The SS versions are required in the non- $W W$ diboson background estimation (see Section 7.2). It does this in a data-driven way by using the $W+$ jet and dijet control samples, subtracting the expected contaminations, and then multiplying by a data-driven fake factor $f_{\ell}$ (defined earlier):

$$
\begin{aligned}
& \mathcal{N}_{\ell \ell, i}^{\text {pred,dijet }}=\left(\mathcal{N}_{\ell \ell, i}^{\text {data }}-\mathcal{N}_{\ell \phi, i}^{\mathrm{MC}, W+\text { jet }}-\mathcal{N}_{\ell \ell, i}^{\mathrm{MC}, \mathrm{EW}}\right) \cdot f_{\ell \mid \ell}^{\text {pred,dijet }} \cdot f_{\ell \mid \ell}^{\text {pred,dijet }} \\
& \mathcal{N}_{\ell \phi, i}^{\text {pred,dijet }}=\left(\mathcal{N}_{\ell \phi, i}^{\text {data }}-\mathcal{N}_{\ell \phi, i}^{\mathrm{MC}, W+\text { jet }}-\mathcal{N}_{\ell \phi, i}^{\mathrm{MC}, \mathrm{EW}}\right) \cdot \sum_{\ell} f_{\ell \mid \ell}^{\text {pred,dijet }} \\
& \mathcal{N}_{\ell \ell, i}^{\text {pred, } W+\text { jet }}=\left(\mathcal{N}_{\ell \ell, i}^{\text {data }}-\mathcal{N}_{\ell \ell, i}^{\text {pred,dijet }}-\mathcal{N}_{\ell \ell, i}^{\mathrm{MC}, \mathrm{EW}}\right) \cdot f_{\ell, i}^{\mathrm{pred}, W+\text { jet }}
\end{aligned}
$$

where $i=\mathrm{OS}, \mathrm{SS}$. In the $e \mu / \mu$ channels, there are in fact two terms because an electron or a muon could be the fake. The dijet contamination to the $W+$ jet control sample $\mathcal{N}_{\ell \ell, i}^{\text {pred,dijet }}$ is data-driven from the dijet control sample. As discussed later, the fake factors are determined by the flavour composition of the jets, and thus depend upon the process. Also, $f_{\ell}^{\text {pred,dijet }}$ depends upon whether the other object in the event is an ID lepton $\left(f_{\ell \mid \ell}^{\text {pred,dijet }}\right)$ or an anti-ID lepton $\left(f_{\ell \mid \ell}^{\text {pred,dijet }}\right)$. This shall be discussed in Section 7.1.6. Finally, note that $f_{\ell}^{\text {pred, } W+\text { jet }}$ depends upon whether the event is OS or SS. This shall be discussed in Section 7.1.5.

An advantage of this sample-based fake factor method compared to that used in the $W W$ cross section measurement (see Section 6.1.5) is that it provides a background estimation in regions other than the signal region.

The rest of this section on the $W+$ jet and dijet backgrounds shall be spent describing the anti-identification lepton selection criteria (Section 7.1.2), the measurement of fake factors in experimental data (Sections 7.1.3 and 7.1.4), and MC-based corrections to these fake factors to improve the background estimations (Sections 7.1.5 and 7.1.6). 


\subsubsection{Lepton anti-identification criteria}

The $\&$ selection criteria are loosened with respect to the $\ell$ selection criteria in order to accept more jets. An explicit veto upon $\ell$ objects avoids overlap between samples.

Relative to the $e$ definition in Section 4.2.3, anti-ID electrons of any $p_{\mathrm{T}}$ must fail the medium identification criteria (though instead must have $n_{\text {pixel }}^{\text {hit }}+n_{\mathrm{SCT}}^{\text {hit }} \geq 4$ ). Also, the tracker and calorimeter isolation are loosened to $p_{\mathrm{T}}^{\text {cone }}(0.3) / E_{\mathrm{T}}<0.16$ and $E_{\mathrm{T}}^{\text {cone }}(0.3) / E_{\mathrm{T}}<0.30$.

Relative to the $\mu$ definition in Section 4.2.4, anti-ID muons have their transverse impact parameter $d_{0}$ requirement removed. Also, the tracker isolation is removed and the calorimeter isolation is loosened to $E_{\mathrm{T}}^{\text {cone }}(0.3) / p_{\mathrm{T}}<0.15$ for $p_{\mathrm{T}} \in[10,15] \mathrm{GeV}$, $E_{\mathrm{T}}^{\text {cone }}(0.3) / p_{\mathrm{T}}<0.25$ for $p_{\mathrm{T}} \in[15,20] \mathrm{GeV}$ and $E_{\mathrm{T}}^{\text {cone }}(0.3) / p_{\mathrm{T}}<0.30$ for $p_{\mathrm{T}}>20 \mathrm{GeV}$.

\subsubsection{Dijet fake factor measurement}

In situ fake factor measurements are made using dijet events. This involves counting the numbers of $\ell$ and $\ell$ objects in a dijet control region (CR), subtracting the expected contamination of prompt leptons from $W$ and $Z$ boson events, and calculating the ratio $f_{\ell}=N_{\ell} / N_{\ell} \cdot f_{\ell}^{\text {data,dijet }}$ is measured as a function of $p_{\mathrm{T}}$ and $\eta$.

Following data quality requirements, events are selected using very loose (no isolation or electron identification criteria), but highly prescaled, lepton triggers. To reduce the effects of prescaling, different triggers were used for different $p_{\mathrm{T}}$ ranges, and a trigger containing electron identification criteria was added to aid measurement of $N_{\ell}$. In the $f_{e}$ measurement, the EF_e5_etcut $\left(0.012 \mathrm{pb}^{-1}\right)$ and $\mathrm{EF} \_$e5_medium1 $\left(0.24 \mathrm{pb}^{-1}\right)$ triggers were used for $p_{\mathrm{T}}<20 \mathrm{GeV}$ and the EF_g24_etcut $\left(2.1 \mathrm{pb}^{-1}\right)$ trigger was used for $p_{\mathrm{T}}>24 \mathrm{GeV}$. In the $f_{\mu}$ measurement, the EF_mu6 $\left(0.94 \mathrm{pb}^{-1}\right)$ trigger was used for $p_{\mathrm{T}}<15 \mathrm{GeV}$ and the EF_mu15 $\left(23 \mathrm{pb}^{-1}\right)$ trigger was used for $p_{\mathrm{T}}>15 \mathrm{GeV}$. The trigger naming scheme is explained in the caption of Table 4.4 .

The dijet CR requires events to have a jet with $p_{\mathrm{T}}>15 \mathrm{GeV}$ (see Section 4.2.5 for jet selection) balancing the triggered lepton object, $\Delta \phi(\ell, j)>0.7$. To suppress contamination from $W$ boson events we require $m_{\mathrm{T}, \ell}<30 \mathrm{GeV}$, and to suppress the $Z$ boson background we veto events with a lepton pair satisfying $\left|m_{\ell \ell}-m_{Z}\right|<13 \mathrm{GeV}$. Note that these criteria apply to both $\ell$ and $\ell$ objects. Normalisation factors for the 

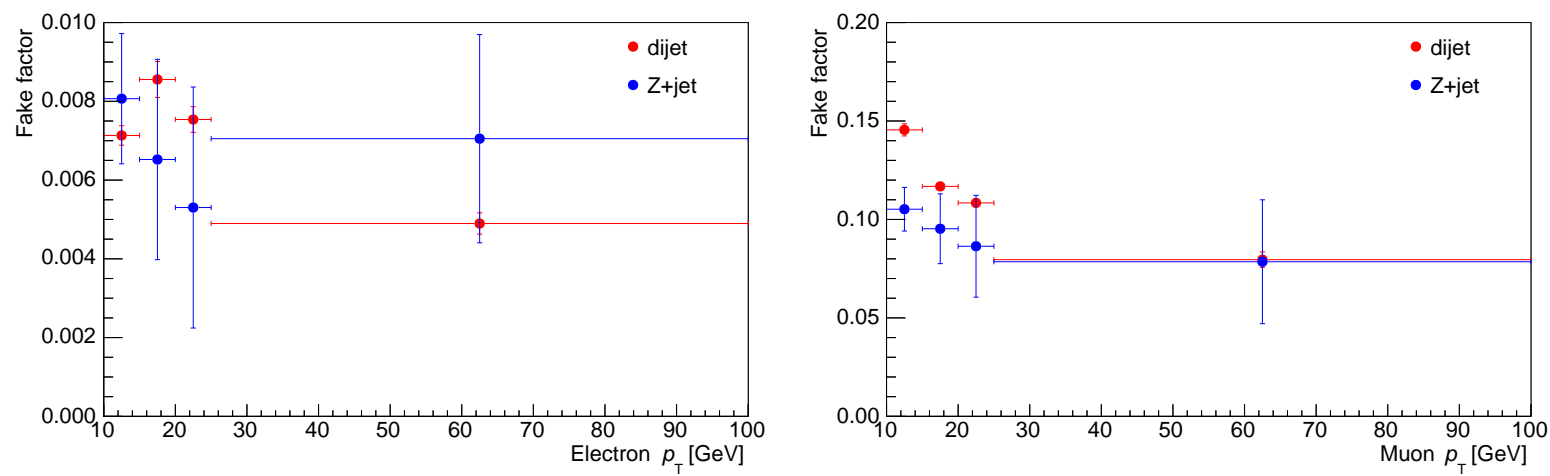

Figure 7.1: The fake factor measured in dijet (red) and $Z+$ jet (blue) events versus $p_{\mathrm{T}}$, for electrons (left) and muons (right) [141]. The error bars include statistical uncertainties and uncertainties in the background subtraction.

MC predictions of the residual $W$ and $Z$ boson backgrounds are derived by inverting the corresponding veto.

The measured electron and muon fake factors are shown in Figure 7.1. The uncertainty is dominated by uncertainties in the subtracted electroweak contamination (which is conservatively scaled up and down by $20 \%$ ). $f_{\ell}^{\text {data,dijet }}$ is used in the dijet background estimation, as described in Section 7.1.6.

\subsubsection{Z+jet fake factor measurement}

In situ fake factor measurements are also made using $Z+$ jet events. This involves counting the numbers of $\ell$ and $\ell$ objects in a $Z+$ jet control region (CR), subtracting the expected prompt leptons and photon conversions from electroweak contamination $(Z \gamma$, $\left.Z Z, Z \gamma^{*}, W \gamma, W Z, W \gamma^{*}\right)$, and calculating their ratio $f_{\ell}=N_{\ell} / N_{\ell}$.

Following data quality requirements, events are selected using unprescaled lepton triggers. This is possible because the triggered object is a lepton from the $Z$ boson decay, and the $p_{\mathrm{T}}$ threshold can therefore be relatively high. In the $f_{e}$ measurement, the EF_e24vhi_medium1 and EF_e60_medium1 triggers support $p_{\mathrm{T}}>25 \mathrm{GeV}$. In the $f_{\mu}$ measurement, the EF_mu24i_tight and EF_mu36_tight triggers are used with the dilepton EF_mu18_tight_mu8_EFFS trigger to support $p_{\mathrm{T}}>22 \mathrm{GeV}$. The trigger naming scheme is explained in the caption of Table 4.4 .

The $Z+$ jet $C R$ requires a pair of same-flavour and oppositely charged $\ell$ objects to reconstruct the $Z$ boson mass, $81<m_{\ell \ell}<107 \mathrm{GeV}$. These leptons are excluded from 
the $f_{\ell}$ calculation. Electroweak contamination is suppressed by cuts on additional $\ell$ and $\&$ objects: $Z Z$ is rejected by a veto on $76<m_{\ell \ell}<107 \mathrm{GeV}$ for additional dilepton systems, and $W Z$ is rejected by $m_{\mathrm{T}, \ell}<30 \mathrm{GeV}$. Residual diboson backgrounds $(Z \gamma$, $\left.Z Z, Z \gamma^{*}, W \gamma, W Z, W \gamma^{*}\right)$ are subtracted using MC predictions.

The measured electron and muon fake factors are shown in Figure 7.1. The uncertainty is dominated by statistical uncertainty. For this reason, $f_{\ell}^{\text {data, } Z+\text { jet }}$ is measured as a function of $p_{\mathrm{T}}$ only, and the $\eta$ dependence is injected from $f_{\ell}^{\text {data,dijet }}$. The uncertainty due to electroweak subtraction is also significant (estimated by varying the diboson cross sections), because the contamination to the $Z+$ jet $\mathrm{CR}$ is not negligible. Although

$f_{\ell}^{\text {data, } Z+\text { jet }}$ has larger uncertainties than $f_{\ell}^{\text {data,dijet }}$, it shall be used in the $W+$ jet background estimation. This is because jets in $Z+$ jet and $W+$ jet events are expected to have similar flavour composition, and therefore similar fake factors (see Section 7.1.5).

\subsubsection{W+jet background estimation}

The $W+$ jet background to the opposite-sign (OS) and same-sign (SS) dilepton samples are estimated from the $W+$ jet control sample, using (7.6). The $W+$ jet control sample $\mathcal{N}_{\text {lq }}$ contains events with one ID lepton and one anti-ID lepton, selected from events passing the data quality criteria and triggers specified in Section 4.3. Generally, it is the lepton from the $W$ boson decay that fires the single lepton triggers. Contamination is small, and is estimated by the fake factor method for dijet events (see Section 7.1.6) and $\mathrm{MC}$ for other processes.

The fake factor of a process is determined by the jet flavour composition of that process. Specifically, $f_{e}$ is larger for heavy flavour because the electron particle identification is tuned to reject light flavour jets, and $f_{\mu}$ is larger for light flavour because the $d_{0}$ criterion suppresses long-lived heavy flavour hadrons. 

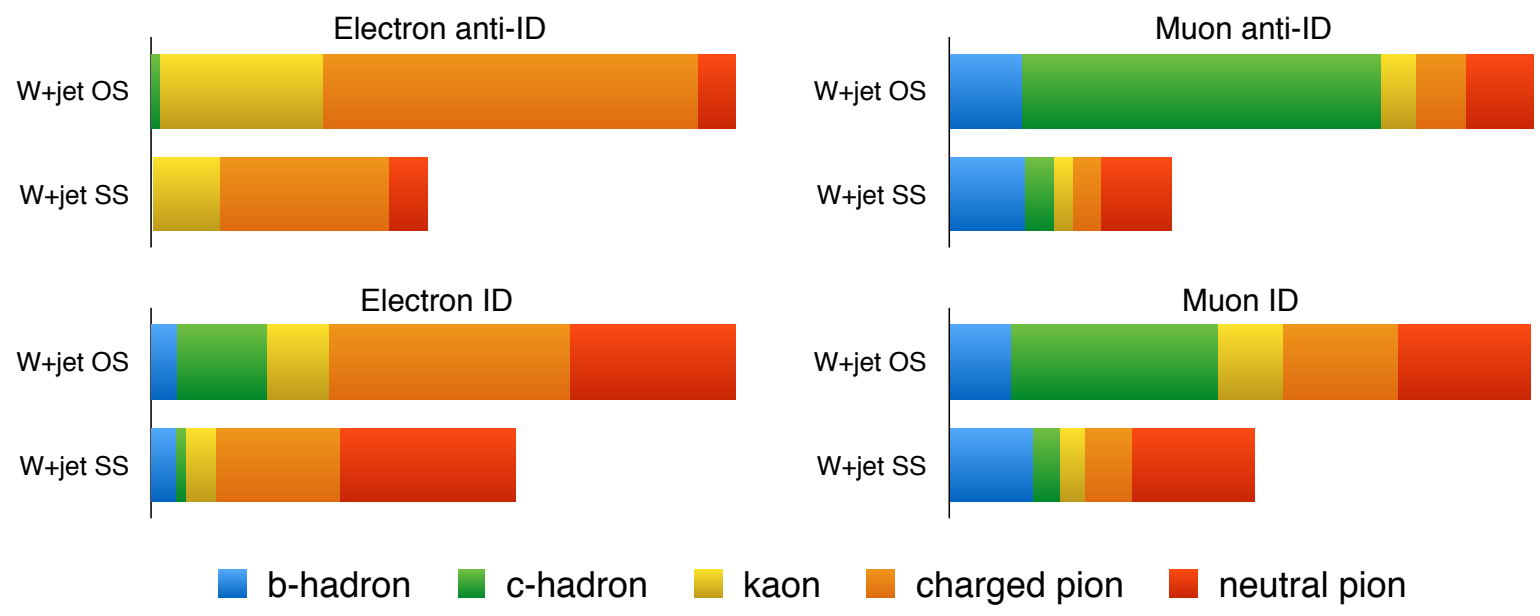

Figure 7.2: The jet flavour composition of fake leptons, for opposite-sign (OS) and same-sign (SS) $W+$ jet events. Each SS axis is scaled to the cross section of the corresponding OS axis.

Since we use both OS and SS samples, it is useful to consider the jet flavour composition of the $W+$ jet process in each sample. There are diagrams like $q g \rightarrow W q^{\prime}$, where the outgoing quark has OS charge to the $W$ boson $($ e.g. $W+c),{ }^{1}$ and there are diagrams like $q \bar{q}^{\prime} \rightarrow W g$, where the gluon subsequently splits to a $q \bar{q}$ pair $(e . g . W+b \bar{b})$. Therefore, in terms of the $W$ and quark charges, the former diagrams contribute to OS only and the latter contribute to OS and SS equally. However, the samples are categorised according to the relative signs of the leptons, and so the extent to which the quark charge is preserved in the reconstructed lepton is important. When a hadron decays leptonically, its sign is preserved in the lepton. Without such a non-prompt lepton, the sign of the jet charge is more likely to be inverted in the reconstructed lepton. In the case of photon conversions from $\pi^{0} \rightarrow \gamma \gamma$, there is no asymmetry. Thus, considering the above points, there is a strong OS/SS asymmetry in $c$-jets, a mild asymmetry in light flavour jets, and no asymmetry in $b$-jets and $\pi^{0} \rightarrow \gamma \gamma$ (see Figure 7.2).

MC-based corrections are applied to the measured $Z+$ jet fake factors in order to account for the different jet flavour compositions of the OS and SS $W+$ jet processes

$$
f_{\ell, i}^{\text {pred, } W+\text { jet }}\left(p_{\mathrm{T}}, \eta\right)=f_{\ell}^{\text {data, } Z+\text { jet }}\left(p_{\mathrm{T}}\right) \cdot \frac{f_{\ell}^{\text {data,dijet }}\left(p_{\mathrm{T}}, \eta\right)}{f_{\ell}^{\text {data,dijet }}\left(p_{\mathrm{T}}\right)} \cdot \frac{f_{\ell, i}^{\mathrm{MC}, W+\text { jet }}}{f_{\ell}^{\mathrm{MC}, Z+\text { jet }}}
$$

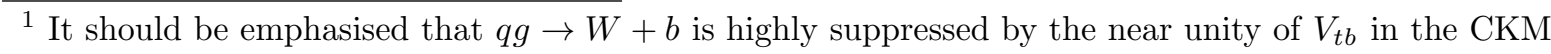
matrix and the negligible top contribution to the incoming PDFs.
} 
where $i=\mathrm{OS}$, SS. The injection of $\eta$-dependence from the dijet fake factor is also shown in (7.7). The $Z+$ jet fake factor is used because $Z+$ jet has a similar jet flavour composition to $W+$ jet. The correction factors are derived with Alpgen+Pythia 6 , and compared to those of Alpgen+Herwig and PowhegBox+Pythia 8 to obtain a systematic uncertainty. The correction factors are $0.99 \pm 0.05$ (stat) \pm 0.19 (syst) for OS electrons, $1.00 \pm 0.08$ (stat) \pm 0.21 (syst) for OS muons, $1.25 \pm 0.08$ (stat) \pm 0.30 (syst) for SS electrons and $1.40 \pm 0.14$ (stat) \pm 0.47 (syst) for SS muons.

The $W+$ jet background is dominated by uncertainties in the fake factor. These are split into components that are correlated and uncorrelated between $f_{\ell, \mathrm{OS}}^{W+\text { jet }}$ and $f_{\ell, \mathrm{SS}}^{W+\text { jet }}$. Components correlated between OS and SS largely cancel in the same-sign control region method of estimating the non- $W W$ diboson background (see Section 7.2.1). This control region also offers validation of the $W+$ jet background estimation.

\subsubsection{Dijet background estimation}

The dijet backgrounds to the dilepton and $W+$ jet samples are estimated from the dijet control sample, using (7.4) and (7.5) respectively. The dijet control sample $\mathcal{N}_{\text {\&f }}$ contains events with two anti-ID leptons, selected from events passing the data quality criteria and triggers specified in Section 4.3. They are accepted by the dilepton triggers since these have looser lepton selections. Contamination from $W+$ jet and other processes is estimated with MC, though is generally small.

The dijet fake factor $f_{\ell}^{\text {data,dijet }}$ is measured in events containing a balancing jet (passing the offline jet selection). However, in (7.4) and (7.5) the fake factors are applied to events containing another anti-ID lepton $\left(f_{\ell \mid \mathcal{f}}^{\text {pred,dijet }}\right)$, or as if there is an ID lepton in the event $\left(f_{\ell \mid \ell}^{\text {pred,dijet }}\right)$. This can heavily bias the jet flavour composition of the selected events, and this in turn can affect the fake factor of interest. For example, if the other object is a muon, this increases the probability that the event contains heavy flavour jets. Consequently, $f_{e}$ would increase and $f_{\mu}$ would decrease (see Section 7.1.5). 

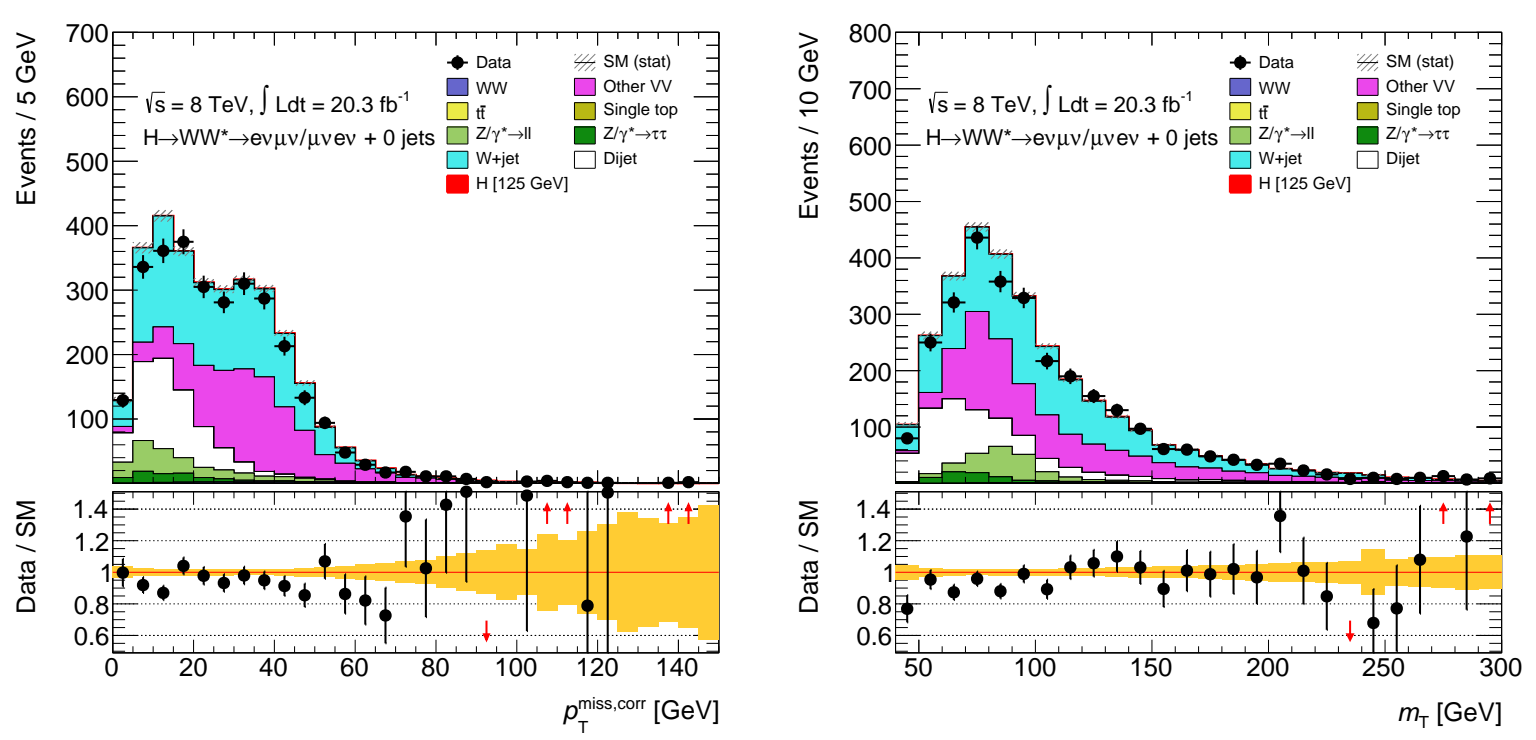

Figure 7.3: The $p_{\mathrm{T}}^{\text {miss,corr }}$ (left) and $m_{\mathrm{T}}$ (right) distributions in the $e \mu / \mu e$ channels of same-sign dilepton events. Selected events have passed the $p_{\mathrm{T}, \ell}^{\text {lead }}>22 \mathrm{GeV}, m_{\ell \ell}>10 \mathrm{GeV}$, $N_{\text {jets }}=0$ and $\Delta \phi\left(\ell \ell, p_{\mathrm{T}}^{\text {inv }}\right)>\pi / 2$ criteria.

MC-based corrections are applied to the measured dijet fake factor in order to account for the correlation between $f_{\ell}$ and the other object in the event

$$
\begin{aligned}
& f_{\ell \mid \ell}^{\text {pred,dijet }}\left(p_{\mathrm{T}}, \eta\right)=f_{\ell}^{\text {data,dijet }}\left(p_{\mathrm{T}}, \eta\right) \cdot \frac{f_{\ell \mid \ell}^{\mathrm{MC}, \text { dijet }}}{f_{\ell \mid j}^{\mathrm{MC}, \mathrm{dijet}}} \\
& f_{\ell \mid \ell}^{\text {pred,dijet }}\left(p_{\mathrm{T}}, \eta\right)=f_{\ell}^{\text {data,dijet }}\left(p_{\mathrm{T}}, \eta\right) \cdot \frac{f_{\ell \mid \ell}^{\mathrm{MC} \text { dijet }}}{f_{\ell \mid j}^{\mathrm{MC}, \mathrm{dijet}}} .
\end{aligned}
$$

The dijet background has a very small contribution to the $H \rightarrow W W$ signal regions, but has large uncertainties dominated by the MC-based corrections. It is difficult to define a high purity dijet validation region in the dilepton sample, though good agreement with experimental data is observed in the low $p_{\mathrm{T}}^{\text {inv }}$ regions where this background is enhanced (see Figure 7.3). 


\subsection{Non-WW diboson}

The non- $W W$ diboson background comprises the $W \gamma, W \gamma^{*}, W Z$ and $Z Z$ processes (in order of contribution to the signal region). $W \gamma$ events feature a prompt photon that passes the electron selection, via an asymmetric conversion $\left(e^{+} e^{-}\right.$production). The other processes have signatures of $\ell \nu \ell \ell, \ell \ell \ell \ell$ or $\ell \ell \nu \nu$, and usually contribute when one or more leptons fail object selection (e.g. $p_{\mathrm{T}}<10 \mathrm{GeV}$ or $|\eta|>2.5$ ).

\subsubsection{Same-sign control region}

In non- $W W$ diboson backgrounds, a symmetry is expected to exist between oppositesign (OS) and same-sign (SS) dilepton events; this is particularly true in the $e \mu / \mu e$ channels, where asymmetric final states are reduced. Conversely, the $W W$, top and $Z / \gamma^{*}$ backgrounds only contribute to the OS sample. Finally, the $W+$ jet background displays a partial OS/SS symmetry, as described in Section 7.1.5. Thus, SS events can be used to estimate the non- $W W$ diboson background in the OS signal region, whilst validating the $W+$ jet estimation.

An SS control region (CR) is defined using identical criteria to the OS signal region (SR). In the language of Section 7.1, the SS CR events are in the SR of the $\mathcal{N}_{\ell \ell, S S}$ sample. This is used to determine the normalisation of the non- $W W$ diboson background, whilst the shapes of observables used in the fitting procedure (i.e. $m_{\mathrm{T}}, m_{\ell \ell}$ and $p_{\mathrm{T}, \ell}^{\text {sublead }}$ ) are modelled by MC. This is equivalent to

$$
\begin{aligned}
N_{V V}^{\mathrm{pred}, \mathrm{SR}} & =\alpha_{V V} \cdot\left(N^{\text {data,CR }}-N_{\mathrm{non}-V V}^{\mathrm{pred}, \mathrm{CR}}\right) \\
\alpha_{V V} & =N_{V V}^{\mathrm{MC}, \mathrm{SR}} / N_{V V}^{\mathrm{MC}, \mathrm{CR}}
\end{aligned}
$$

where $V V=W \gamma+W \gamma^{*}+W Z+Z Z$, and $N_{\text {non- } V V}^{\text {pred } V}$ is dominated by $W+$ jet. The extrapolation $\alpha_{V V}$ represents the MC predictions for shapes of observables, since the OS/SS symmetry implies that the total normalisation is largely unchanged.

The SS CR method is only used in the 0-jet and 1-jet bins of the $e \mu / \mu e$ channels, using a combined $e \mu+\mu e$ channel to define the CR. All other signal regions estimate the non- $W W$ diboson background using MC only.

Uncertainties in the normalisation of the constituent processes will cancel if the composition of the non- $W W$ diboson background is the same in SS and OS events; this is 
modelled by MC. However, uncertainties in the shapes of distributions remain, since these are not constrained by the SS CR method. ${ }^{2}$ Additionally, the uncertainty component of the OS $W+$ jet background that is correlated between OS and SS will cancel in the SS CR method; an increase in the SS $W+$ jet background will be compensated by a decrease in the non- $W W$ diboson background prediction, and vice versa.

Figure 7.4 validates the MC shape modelling of the fit observables in the SS CRs, following application of the normalisation factors. Good agreement with experimental data is observed. Note that these SS distributions are not directly used, since only the total number of events in the SS CRs are used in (7.10).

\subsection{2 $\mathrm{W} \gamma$}

$W \gamma$ events enter the dilepton sample when the photon fakes an electron. This is usually caused by an asymmetric $\gamma \rightarrow e^{+} e^{-}$conversion, where only one electron is reconstructed in the tracker and calorimeter. This background is suppressed by the electron identification criteria, which require a hit in the first pixel layer and no conversion vertex (see Section 4.2.3).

$W \gamma$ is modelled by ALPGEn+Herwig and normalised to the NLO cross section calculated with MCFM. Modelling is tested with a SS dilepton sample of $e \mu / \mu e$ events, but where the electron object is required to have a conversion vertex and no hit in the first pixel layer (i.e. the photon conversion rejection criteria are inverted). Validation regions (VRs) are defined in the 0-jet and 1-jet bins, using the corresponding signal region cuts. Experimental data are well described, as seen in Figure 7.5.

As the conversion and pixel hit criteria are inverted in the VR, their SR modelling is not tested in Figure 7.5. Unfortunately, it is not possible to define a high-purity $W \gamma$ VR when including these criteria. Instead, a $Z \rightarrow \mu \mu \gamma \mathrm{VR}$ is used to test this modelling. Events are selected with an OS muon pair with $p_{\mathrm{T}, \mu}^{\text {lead }}>22 \mathrm{GeV}$ and $p_{\mathrm{T}, \mu}^{\text {sublead }}>10 \mathrm{GeV}$, and an additional electron candidate with $p_{\mathrm{T}, e}>10 \mathrm{GeV}$. Low mass resonances are vetoed by $m_{\mu \mu}>12 \mathrm{GeV}$, and events featuring QED FSR are selected by $\left|m_{\mu \mu e}-m_{Z}\right|<15 \mathrm{GeV}$. The selected events are $\sim 55 \% Z \gamma$ and $\sim 45 \% W Z / Z \gamma^{*}$. A mismodelling is found depending upon $p_{\mathrm{T}, e}$ and so a conversion systematic uncertainty is derived: $25 \%$ for $10-15 \mathrm{GeV}, 18 \%$ for $15-20 \mathrm{GeV}$ and $5 \%$ for $>20 \mathrm{GeV}$.

\footnotetext{
${ }^{2}$ Shape uncertainties are also introduced by normalisation uncertainties in the individual processes comprising the non- $W W$ diboson background, which lead to a composition uncertainty.
} 

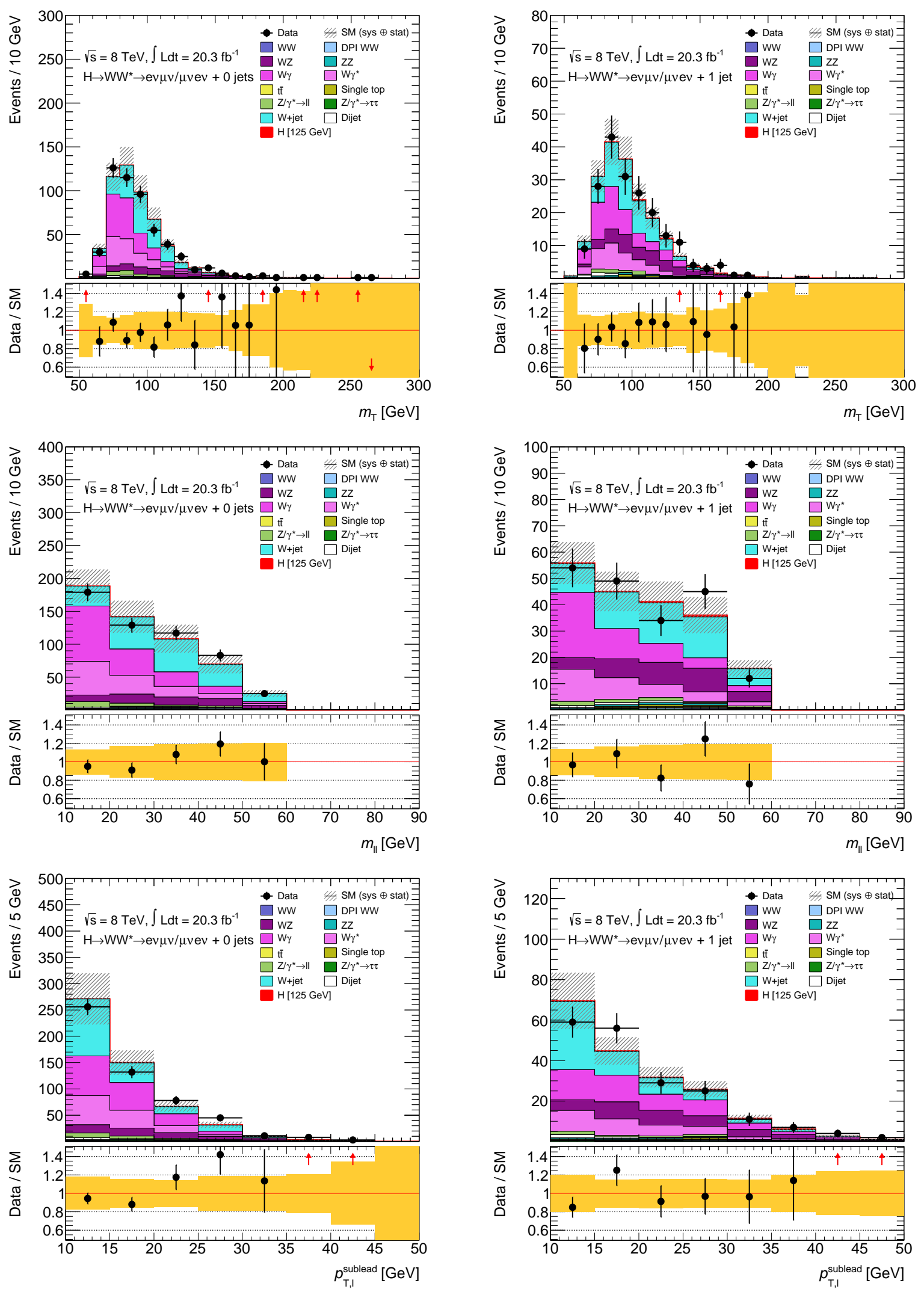

Figure 7.4: The $m_{\mathrm{T}}$ (top), $m_{\ell \ell}$ (middle) and $p_{\mathrm{T}, \ell}^{\text {sublead }}$ (bottom) distributions in the 0-jet (left) and 1-jet (right) same-sign control regions. Normalisation factors are applied. 

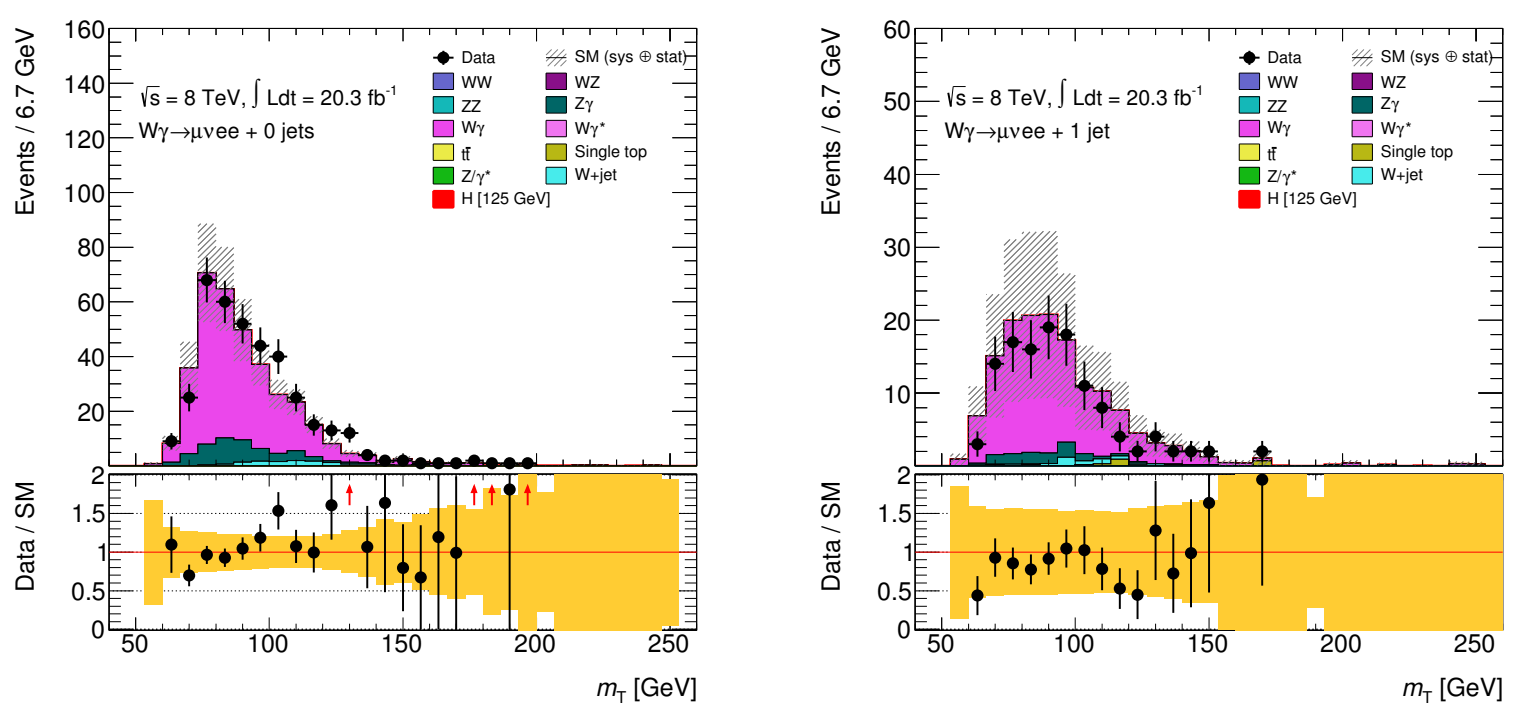

Figure 7.5: The $m_{\mathrm{T}}$ distribution in the 0-jet (left) and 1-jet (right) $W \gamma$ validation regions [141]. The shaded error band includes statistical and theoretical uncertainties, in addition to those associated with conversion modelling.

\subsubsection{WZ and $\mathrm{W} \gamma^{*}$}

Both the $W Z$ and $W \gamma^{*}$ processes result in a $\ell \nu \ell^{\prime} \ell^{\prime}$ final state, peaking in $m_{\ell^{\prime} \ell^{\prime}}$ at $m_{Z}$ and $2 m_{\ell^{\prime}}$ respectively (due to the different $Z$ and $\gamma$ propagators). Their interference at intermediate $m_{\ell^{\prime} \ell^{\prime}}$ is non-negligible, and so these processes are generated together.

It is technically difficult to generate $\mathrm{MC}$ events with very low $m_{\ell^{\prime} \ell^{\prime}}$. For this reason, the phase space is generated in two parts: high mass " $W Z$ " events with $m_{\ell^{\prime} \ell^{\prime}}>7 \mathrm{GeV}$ and low mass " $W \gamma^{*}$ " events with $m_{\ell^{\prime} \ell^{\prime}}<7 \mathrm{GeV} .{ }^{3}$ In ambiguous cases $\left(\ell=\ell^{\prime}\right)$, the lowest mass opposite-sign same-flavour dilepton pair is used to split the phase space. $W Z$ is modelled at NLO by PowhegBox+Pythia 8.

$W \gamma^{*}$ is technically difficult to model because it involves integrating over a phase space with extremely low mass; the threshold for production is $m_{\ell^{\prime} \ell^{\prime}}=2 m_{\ell^{\prime}}$, which is $1.022 \mathrm{MeV}$ for $\ell$ vee. In previous analysis iterations [8], $W \gamma^{*}$ was modelled by MADGRAPH+PYTHIA 6 . However, this implementation is not designed to produce gauge-invariant results when $m_{\ell^{\prime} \ell^{\prime}} \ll \Gamma_{W}$ [142], and numerical limitations result in wildly unphysical events in this region of phase space (e.g. leptons with $p_{\mathrm{T}} \sim 1 \mathrm{TeV}$ ). In an attempt to resolve this problem, events with $m_{\ell^{\prime} \ell^{\prime}}<3 \mathrm{MeV}$ were removed from the MC sample, and events

\footnotetext{
${ }^{3}$ It is these " $W Z$ " and " $W \gamma^{*}$ " MC samples that are referred to as the $W Z$ and $W \gamma^{*}$ backgrounds, though each sample contains both the $W Z$ and $W \gamma^{*}$ processes and all interference.
} 
with $3 \mathrm{MeV}<m_{\ell^{\prime} \ell^{\prime}}<10 \mathrm{MeV}$ were reweighted in order to recover the cross section. Even with this fix, this background was associated with a modelling uncertainty of $40 \%$ (evaluated by comparing to SHERPA).

SHERPA can produce gauge-invariant results for the full mass range, since it employs a complex mass scheme [143]. A simple LO SHERPA sample underestimates the FSR phase space with $p_{\mathrm{T}, \ell^{\prime} \ell^{\prime}}>m_{W} / 2$. For this reason, $W \gamma^{*}$ is modelled by ME-PS merging with up to one additional parton (see Section 2.2.3). It is not currently possible to include further partons in the ME-PS merging over the full mass range.

The SHERPA sample is normalised using an NLO $K$-factor of $0.94 \pm 0.07$ (scale), calculated with MCFM. Due to technical limitations, this is calculated in a high mass region $m_{\ell^{\prime} \ell^{\prime}} \in[0.5,7] \mathrm{GeV}$ and then extrapolated down in mass. It is calculated with the $p_{\mathrm{T}, \ell}^{\text {lead }}>22 \mathrm{GeV}$ and $p_{\mathrm{T}, \ell}^{\text {sublead }}>10 \mathrm{GeV}$ criteria.

Since the $W \gamma^{*}$ acceptance of the lepton $p_{\mathrm{T}}$ criteria is strongly related to $N_{\text {jets }}$ and because the $H \rightarrow W W$ analysis itself is jet binned, the jet multiplicity distribution must be well modelled. For this reason, the $N_{\text {jets }}$ distribution is reweighted to that of a SHERPA ME-PS merged sample with up to two additional partons, using corrections of $0.91 \pm 0.06$ (scale) in the 0-jet bin, $1.09 \pm 0.33$ (scale) in the 1-jet bin, and $2.0 \pm 0.5$ (scale) in the $\geq 2$-jet bin. Again, due to technical limitations, this is calculated in a high mass region $m_{\ell^{\prime} \ell^{\prime}} \in[0.5,7] \mathrm{GeV}$ and then extrapolated down in mass. It is also calculated with the $p_{\mathrm{T}, \ell}^{\text {lead }}>22 \mathrm{GeV}$ and $p_{\mathrm{T}, \ell}^{\text {sublead }}>10 \mathrm{GeV}$ criteria.

Two sources of theoretical uncertainties in the signal region acceptances are considered: higher order corrections, estimated by varying $\mu_{\mathrm{R}}$ and $\mu_{\mathrm{F}}$ as described elsewhere in this thesis; and modelling uncertainties, evaluated by comparing the SHERPA ME-PS samples with $\leq 1$ parton (the default) and $\leq 2$ partons in a high mass region $m_{\ell^{\prime} \ell^{\prime}} \in[0.5,7] \mathrm{GeV}$. The uncertainty in the selection acceptance are negligible compared to the theoretical uncertainties in the $K$-factor and jet-bin correction. Scale uncertainties in the shape of the $m_{\mathrm{T}}$ distribution are also evaluated.

The $W \gamma^{*}$ modelling is tested in a $W \gamma^{*} \rightarrow e \nu \mu \mu$ validation region (VR). Since $\Delta R(\mu, \mu)$ is generally small, the muon isolation criteria are altered: tracks associated with other muons are removed from the $p_{\mathrm{T}}^{\text {cone }}$ definition, and the calorimeter isolation is loosened to $E_{\mathrm{T}}^{\text {cone }}(0.3) / p_{\mathrm{T}}<0.4$ for $p_{\mathrm{T}}<15 \mathrm{GeV}$ (c.f. Section 4.2.4). Events are selected with $p_{\mathrm{T}, e}>22 \mathrm{GeV}, p_{\mathrm{T}, \mu}^{\text {lead }}>10 \mathrm{GeV}$ and $p_{\mathrm{T}, \mu}^{\text {sublead }}>3 \mathrm{GeV}$. Then, the $\mathrm{VR}$ is defined by $m_{\mu \mu}<7 \mathrm{GeV},\left|m_{\mu \mu}-m_{J / \psi}\right|>100 \mathrm{MeV}, E_{\mathrm{T}, \text { rel }}^{\text {miss }}>20 \mathrm{GeV}$ and $\max (\Delta \phi(\ell, \ell))<2.8$. The experimental data in the VR is well described by SHERPA, as seen in Figure 7.6, 

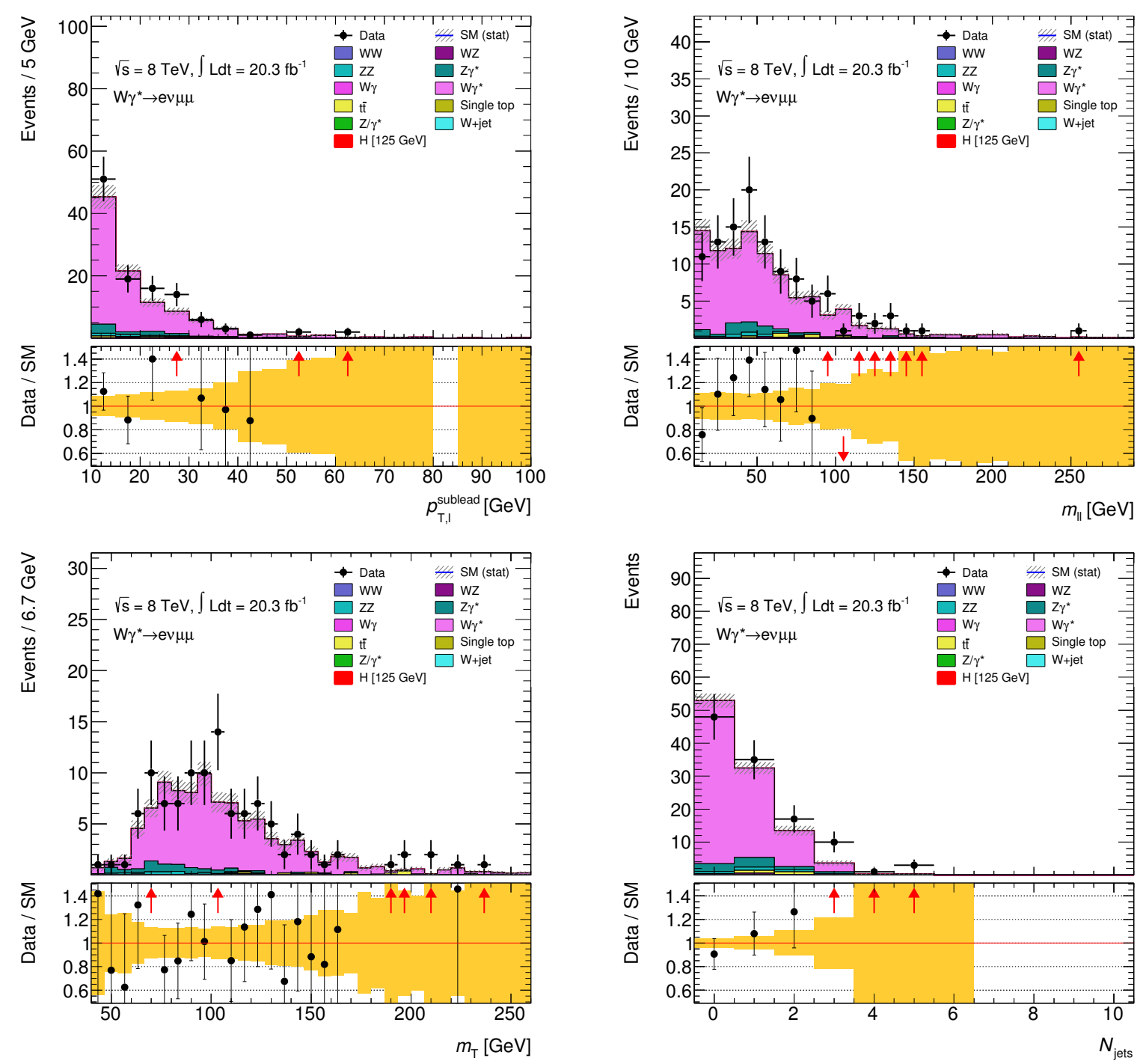

Figure 7.6: The $p_{\mathrm{T}, \ell}^{\text {sublead }}$ (top left), $m_{\ell \ell}$ (top right), $m_{\mathrm{T}}$ (bottom left) and $N_{\text {jets }}$ (bottom right) distributions in the $W \gamma^{*}$ validation region [141]. Leptonic observables are defined with the electron and the leading muon.

and supports the normalisation used. It appears that $N_{\text {jets }}$ is underestimated, though this effect is within theoretical uncertainties (which are not shown in Figure 7.6).

It should be noted that the $W \gamma^{*} \mathrm{VR}$ does not test the modelling of $W \gamma^{*} \rightarrow \ell \nu e e$, which can contribute background events via an additional mechanism: both electrons can be reconstructed as a single electron when $\Delta R(e, e)$ is very small. However, the good agreement in the same-sign control region suggests that this is well-modelled. 


\subsection{4 $\mathrm{ZZ}$ and $\mathrm{Z} \gamma^{*}$}

Similar to Section 7.2.3, the $Z Z$ and $Z \gamma^{*}$ processes share a $\ell \ell \ell^{\prime} \ell^{\prime}$ final state, and are modelled together in order to include their interference. Again, the phase space is split: high mass " $Z Z$ " events with $m_{\ell \ell}>4 \mathrm{GeV}$ and $m_{\ell^{\prime} \ell^{\prime}}>4 \mathrm{GeV}$, and low mass " $Z \gamma^{*}$ " events with $m_{\ell \ell}>4 \mathrm{GeV}$ and $m_{\ell^{\prime} \ell^{\prime}}<4 \mathrm{GeV}$. The corresponding " $\gamma^{*} \gamma^{*}$ " process is not modelled due to technical limitations. In ambiguous cases $\left(\ell=\ell^{\prime}\right)$, the lowest mass opposite-sign same-flavour dilepton pair is used to split the phase space. The $\ell \ell \nu^{\prime} \nu^{\prime}$ final state is also considered, though its contribution is negligible.

$Z Z$ is modelled at NLO by PowhegBox+Pythia 8 and the NNLO $g g \rightarrow Z Z$ diagrams are also modelled by GG2zz+HERwiG. $Z \gamma^{*}$ is modelled by SHERPA and is normalised using an NLO $K$-factor of $0.88 \pm 0.05$ (scale), calculated with MCFM. Neither background contributes significantly to the signal region. However, it is important to model the $Z \gamma^{*}$ background in the $Z \gamma$ validation region (see Section 7.2.2) and when measuring the $Z+$ jet fake factor (see Section 7.1.4).

\subsection{Top}

The leading and subleading contributions to the top background are the $t \bar{t}$ and $t W$ processes, respectively. These are irreducible backgrounds, in that they both exhibit the opposite-sign dilepton $+p_{\mathrm{T}}^{\text {inv }}$ experimental signature. This signature results from the top decays, $\mathrm{BR}(t \rightarrow W b) \approx 100 \%$ (occurring before hadronisation), followed by leptonic $W$ boson decays.

The $b$-jets present in top events motivate the jet binning of the $H \rightarrow W W$ analysis (see Figure 4.12). Jets with a $p_{\mathrm{T}}$ threshold of 25 (30) $\mathrm{GeV}$ in the central (forward) region are used for this binning (see Section 4.2.5). The top background is further discriminated by counting the number of $b$-tagged jets with $p_{\mathrm{T}}>20 \mathrm{GeV}$ in the central region, using an algorithm with a tagging efficiency of $85 \%$ (see Section 4.2 .6 ). In the 1 -jet and $\geq 2$-jet bins, the top background is suppressed by vetoing events with $b$-tagged jets.

$t \bar{t}, t W$ and $s$-channel single top $(t b)$ are modelled by PowhegBox+Pythia 6 , while $t$ channel single top $(t b q)$ is modelled by ACERMC+PYTHIA 6. However, the jet binning and

\footnotetext{
${ }^{4}$ It is these " $Z Z$ " and " $Z \gamma^{*}$ " MC samples that are referred to as the $Z Z$ and $Z \gamma^{*}$ backgrounds, though each sample contains the $Z Z, Z \gamma^{*}$ and $\gamma^{*} \gamma^{*}$ processes and all interference.
} 
$b$-tagged jet veto introduce large modelling uncertainties, and so data-driven techniques are used to estimate this background.

\subsubsection{0-jet bin estimation}

In the 0 -jet bin, the top background is very small because both $b$-jets must fail the jet selection. It is estimated by the data-driven jet veto survival probability method.

An extended signal region (ESR) is defined by the pre-selection in Section 4.3.3, with an additional $\Delta \phi(\ell, \ell)<2.8$ criterion to suppress the $Z / \gamma^{*} \rightarrow \tau \tau$ background. The aim is to estimate the number of events in the ESR passing the jet veto $N_{\text {top }}^{\text {pred,ESR,0j }}$, and then extrapolate to the 0-jet signal region (SR) with $\mathrm{MC}$

$$
\begin{aligned}
N_{\mathrm{top}}^{\mathrm{pred}, \mathrm{SR}, 0 \mathrm{j}} & =\alpha_{\mathrm{top}}^{0 \mathrm{j}} \cdot \epsilon_{0, \mathrm{top}}^{\mathrm{pred}, \mathrm{ESR}} \cdot\left(N^{\mathrm{data}, \mathrm{ESR}}-N_{\text {non-top }}^{\mathrm{pred}, \mathrm{ESR}}\right) \\
\alpha_{\mathrm{top}}^{0 \mathrm{j}} & =N_{\mathrm{top}}^{\mathrm{MC}, \mathrm{SR}, 0 \mathrm{j}} / N_{\mathrm{top}}^{\mathrm{MC}, \mathrm{ESR}, 0 \mathrm{j}}
\end{aligned}
$$

where $\epsilon_{0}$ is the jet veto efficiency. Figure 4.12 shows that, after pre-selection, top dominates the $e \mu / \mu e$ channels, but $Z / \gamma^{*} \rightarrow \ell \ell$ dominates the $e e / \mu \mu$ channels. For this reason, the top normalisation is derived from a combined $e \mu+\mu e$ channel and extrapolated to each $e \mu / \mu e / e e+\mu \mu \mathrm{SR}$.

The ESR is dominated by $t \bar{t}$ events featuring two $b$-jets. Since each $b$-quark originates from the decay of a top quark, the kinematic distributions of the two jets are similar. Thus, it is possible to suppress systematic uncertainties by correcting the MC using the second jet veto efficiency $\epsilon_{1}$ measured in a top control region (CR). The square of the data-driven correction to $\epsilon_{1, \text { top }}$ is used to correct the $\epsilon_{0, \text { top }}$ modelled by MC in the ESR:

$$
\epsilon_{0, \text { top }}^{\text {pred,ESR }}=\epsilon_{0, \text { top }}^{\mathrm{MCSR}}\left(\frac{\epsilon_{1}^{\mathrm{data}, \mathrm{CR}}}{\epsilon_{1, \mathrm{top}}^{\mathrm{MC}, \mathrm{CR}}}\right)^{2} .
$$

The top CR requires at least one $b$-tagged jet with $p_{\mathrm{T}}>25 \mathrm{GeV}$. Then $\epsilon_{1}^{\text {data,CR }}$ is measured by counting the fraction of $\mathrm{CR}$ events with zero additional jets. These are defined as jets with $\Delta R>1$ from the highest scoring $b$-tagged jet (see Figure 7.7). It is found that (7.14) results in reduced uncertainties even when considering contributions from other top processes and jets other than the two $b$-jets. 

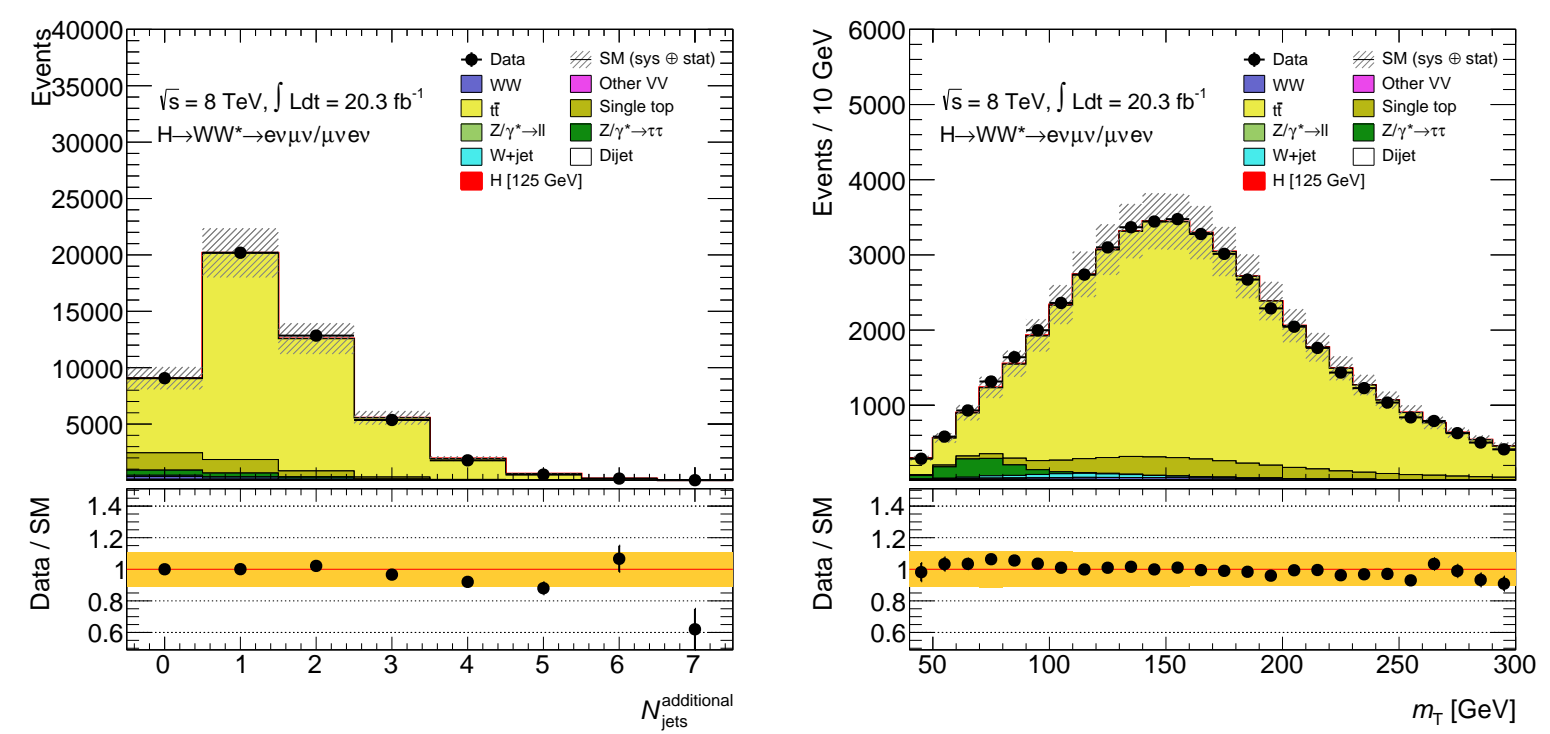

Figure 7.7: The number of additional jets (left) and the $m_{\mathrm{T}}$ distribution (right) in the top control region used by the jet veto survival probability method. The fraction of events with zero additional jets is $\epsilon_{1, \text { top }}^{\text {data,CR }}$, as described in the text.

The total uncertainties in the expected 0 -jet top background is $\sim 8 \%$, dominated by theoretical uncertainties in the extrapolation $\alpha_{\text {top }}^{0 \mathrm{j}}$ and JES/JER uncertainties in the predicted jet veto efficiency $\epsilon_{0, \text { top }}^{\text {pred,ESR }}$.

\subsubsection{1-jet bin estimation}

In the 1-jet bin, the top background is suppressed by removing events with a $b$-tagged jet with $p_{\mathrm{T}}>20 \mathrm{GeV}$. Since the $b$-tagging efficiency is associated with large uncertainties, the data-driven jet b-tagging efficiency extrapolation method is used to estimate this background.

An extended signal region (ESR) is defined by the pre-selection in Section 4.3.3. Since it is the $N_{\text {jets }}=1$ selection and the $b$-tagged jet veto that introduce the largest uncertainties to this background, the aim of the method is to estimate the number of

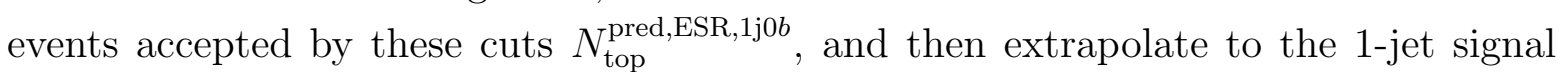
region (SR) with $\mathrm{MC}$

$$
\begin{aligned}
N_{\text {top }}^{\text {pred,SR, } 1 \mathrm{j} 0 b} & =\alpha_{\text {top }}^{1 \mathrm{j}} \cdot N_{\text {top }}^{\text {pred,ESR, } 1 \mathrm{j} 0 b} \\
\alpha_{\text {top }}^{1 \mathrm{j}} & =N_{\text {top }}^{\mathrm{MC}, \mathrm{SR}, 1 \mathrm{j} 0 b} / N_{\text {top }}^{\mathrm{MC}, \mathrm{ESR}, 1 \mathrm{j} 0 b} .
\end{aligned}
$$



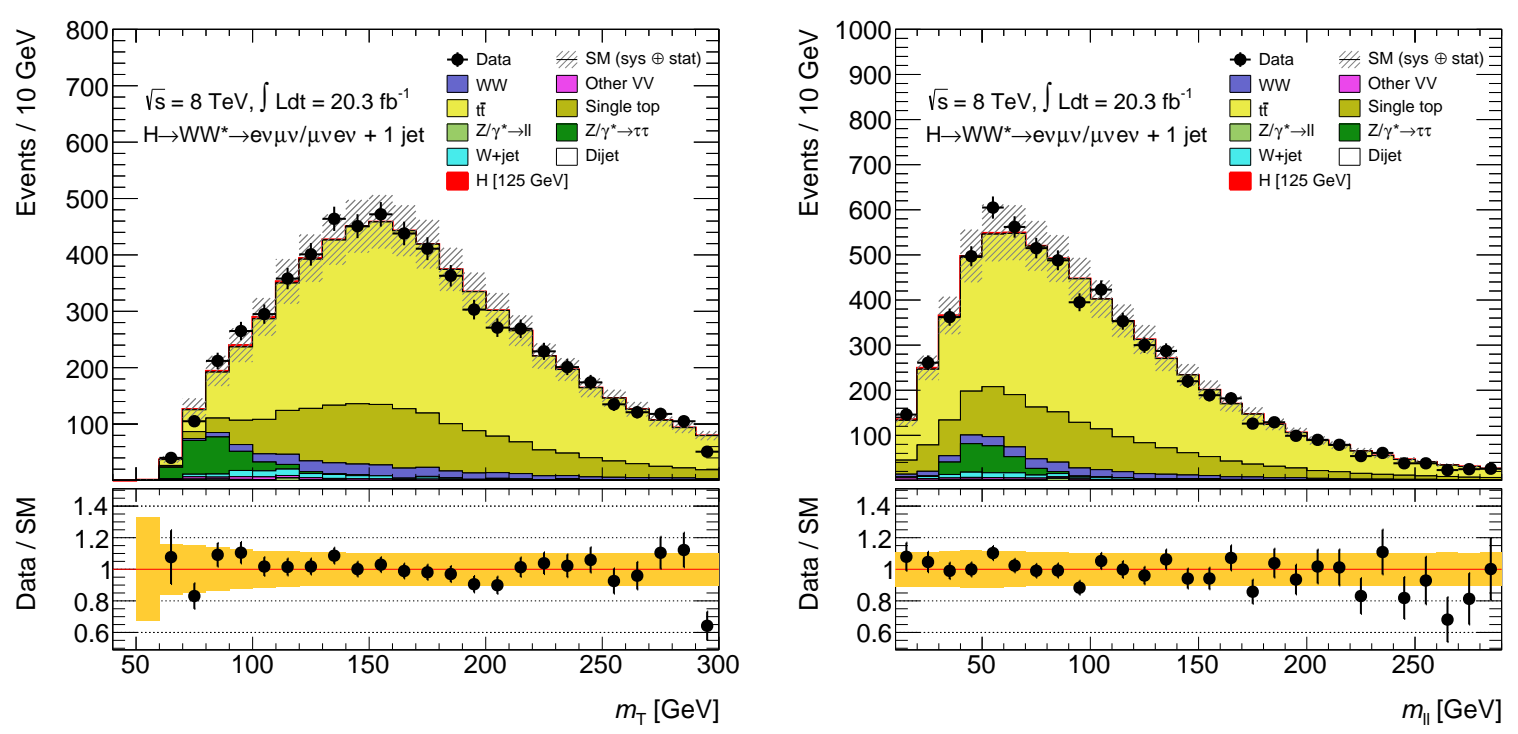

Figure 7.8: The $m_{\mathrm{T}}$ (left) and $m_{\ell \ell}$ (right) distributions for events passing the pre-selection and containing $1 b$-tagged jet. The jet $b$-tagging efficiency extrapolation method uses the top normalisation in this region and extrapolates to events containing 1 jet that is not $b$-tagged. Normalisation factors are applied.

The top normalisation is derived from a combined $e \mu+\mu e$ channel and extrapolated to each $e \mu / \mu e / e e+\mu \mu \mathrm{SR}$.

In order to estimate $N_{\text {top }}^{\text {pred,ESR,1j0b }}$, the number of 1-jet events in the ESR where the jet is $b$-tagged $N^{\text {data,ESR, } 1 \mathrm{j} 1 b}$ is measured, which is highly pure in top events (see Figure 7.8). Then the non-top contamination is subtracted, and the $b$-tagged jet selection is inverted

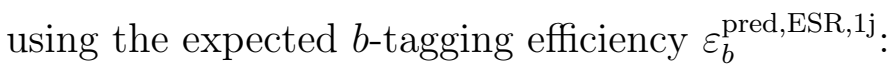

$$
N_{\mathrm{top}}^{\mathrm{pred}, \mathrm{SR}, 1 \mathrm{j} 0 b}=\alpha_{\mathrm{top}}^{1 \mathrm{j}} \cdot \frac{1-\varepsilon_{b}^{\mathrm{pred}, \mathrm{ESR}, 1 \mathrm{j}}}{\varepsilon_{b}^{\text {pred,ESR,1j }}} \cdot\left(N^{\mathrm{data}, \mathrm{ESR}, 1 \mathrm{j} 1 b}-N_{\text {non-top }}^{\text {pred,ESR } 1 \mathrm{j} 1 b}\right)
$$

It should be emphasised that $\varepsilon_{b}$ is a per-jet efficiency, rather than a per-event efficiency (c.f. $\left.\epsilon_{0}\right)$. Also, $\varepsilon_{b}$ is the average $b$-tagging efficiency for a jet in a top event, and will include non- $b$-jets (i.e. it is a mixture of tagged $b$-jets and mis-tagged other jets).

The $b$-tagging efficiency of jets in top events $\varepsilon_{b}$ is measured in a high-purity top control region (CR). These events pass the pre-selection (minus the $p_{\mathrm{T}}^{\text {miss,corr }}$ cut) and feature two jets, at least one of which is $b$-tagged. $\varepsilon_{b}$ is then measured using a tag-and-probe method (see Section 4.2.3), where the tag is a $b$-tagged jet and the probe is the other jet. Since $\varepsilon_{b}$ is measured in 2-jet events but applied to 1 -jet events, an MC-based correction 
is applied to the measured $b$-tagging efficiency

$$
\varepsilon_{b}^{\mathrm{pred}, \mathrm{ESR}, 1 \mathrm{j}}=\frac{\varepsilon_{b}^{\mathrm{MC}, \mathrm{ESR}, 1 \mathrm{j}}}{\varepsilon_{b}^{\mathrm{MC}, \mathrm{CR}, 2 \mathrm{j}}} \cdot \varepsilon_{b}^{\mathrm{data}, \mathrm{CR}, 2 \mathrm{j}} .
$$

The total uncertainty in the expected 1-jet top background is $\sim 7 \%$, dominated by theoretical uncertainties in the MC-based correction to $\varepsilon_{b}$ in (7.18).

\subsection{3 $\geq 2$-jet bin estimation}

As in the 1-jet bin, a veto on b-tagged jets rejects the majority of the top background. However, even after this veto, top remains the largest background in the $\geq 2$-jet bin. Fortunately, this enables a top control region (CR) to be defined which includes the $b$-tagged jet veto, greatly reducing the uncertainties due to $b$-tagging efficiencies.

The top CR is defined in a high- $m_{\ell \ell}$ region, similarly to the $W W$ CRs in the 0-jet and 1 -jet bins. It is defined by the same criteria as the $\geq 2$-jet $\mathrm{SR}$ (minus the $m_{\tau \tau}, \Delta \phi(\ell, \ell)$ and $V H$ cuts), but the $m_{\ell \ell}$ cut is changed to $m_{\ell \ell}>80 \mathrm{GeV}$. The observed number of events is then extrapolated to the signal region (SR) using $\mathrm{MC}$

$$
\begin{aligned}
N_{\text {top }}^{\text {pred,SR }} & =\alpha_{\text {top }}^{\geq 2 \mathrm{j}}\left(N^{\text {data,CR }}-N_{\text {non-top }}^{\text {pred,CR }}\right) \\
\alpha_{\text {top }}^{\geq 2 \mathrm{j}} & =N_{\text {top }}^{\mathrm{MC}, \mathrm{SR}} / N_{\text {top }}^{\mathrm{MC}, \mathrm{CR}} .
\end{aligned}
$$

The uncertainty is dominated by theoretical uncertainties in the extrapolation $\alpha_{\text {top }}^{\geq 2 j}$. Figure 7.9 shows the good description of experimental data in the CR, after application of the normalisation factors. 

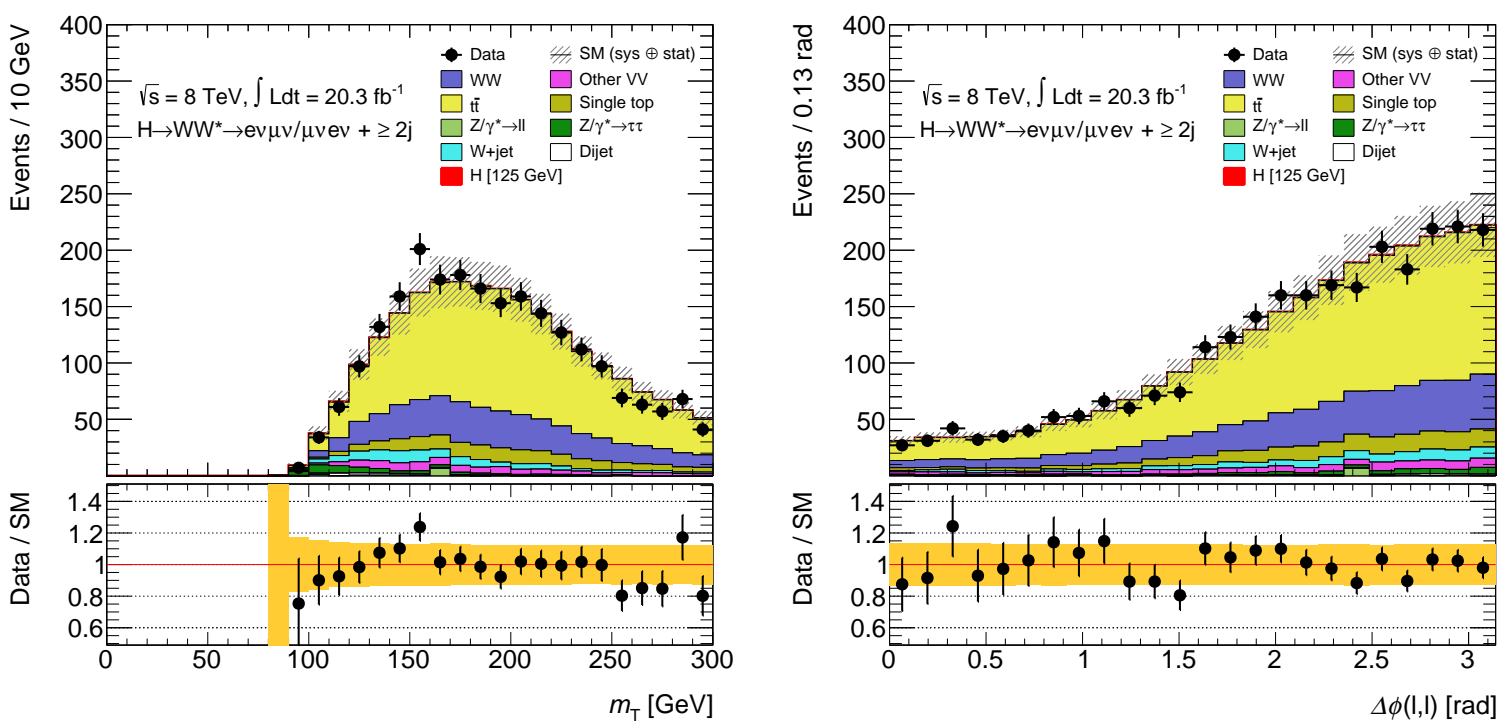

Figure 7.9: The $m_{\mathrm{T}}$ (left) and $\Delta \phi(\ell, \ell)$ (right) distributions in the top control region of the $\geq 2$-jet bin. Normalisation factors are applied. 


\section{$7.4 \mathrm{Z} / \gamma^{*}$}

The $Z / \gamma^{*}$ background is naturally split into $Z / \gamma^{*} \rightarrow \ell \ell$ and $Z / \gamma^{*} \rightarrow \tau \tau$ processes. The former contributes almost exclusively to the $e e / \mu \mu$ channels,${ }^{5}$ where it is the dominant background. The latter can contribute to any channel since the two $\tau \rightarrow \ell \nu_{\ell} \nu_{\tau}$ decays are independent, though is doubly suppressed by the small $\operatorname{BR}\left(\tau \rightarrow \ell \nu_{\ell} \nu_{\tau}\right)=17.6 \%$ [26].

Although $Z / \gamma^{*} \rightarrow \ell \ell$ does not feature prompt neutrinos, degradation of the $p_{\mathrm{T}}^{\text {inv }}$ resolution due to high pile-up can cause some $Z / \gamma^{*} \rightarrow \ell \ell$ events to exhibit significant $p_{\mathrm{T}}^{\mathrm{inv}}$. Since $Z / \gamma^{*} \rightarrow \ell \ell$ is the dominant background in the ee/ $\mu \mu$ channels, a high threshold of $E_{\mathrm{T}, \mathrm{rel}}^{\text {miss }}>40 \mathrm{GeV}$ is used in the pre-selection, which is further tightened by trackerbased $p_{\mathrm{T}, \mathrm{mel}}^{\text {miss }}$ cuts. On the other hand, $Z / \gamma^{*} \rightarrow \tau \tau$ does feature prompt neutrinos and is suppressed by other cuts, such as the $m_{\tau \tau}$ veto.

The $Z / \gamma^{*}$ backgrounds are estimated by data-driven techniques, in combination with MC modelling provided by Alpgen+Herwig. Overlap between the $Z / \gamma^{*} \mathrm{MC}$ and the $Z \gamma \mathrm{MC}$ is removed through careful consideration of the $\mathrm{MC}$ event records.

\subsubsection{Z/ $\gamma^{*}$ boson transverse momentum}

Selecting $e e / \mu \mu$ events with $\left|m_{\ell \ell}-m_{Z}\right|<15 \mathrm{GeV}$ results in a very pure sample of $Z / \gamma^{*} \rightarrow \ell \ell$ events, enabling the MC to be validated. In doing so, the $p_{\mathrm{T}, \ell \ell}$ distribution is found to be poorly modelled in the 0 -jet bin for $p_{\mathrm{T}, \ell \ell}>30 \mathrm{GeV}$ (see Figure 7.10), despite being well modelled inclusively. This is unsurprising: by vetoing events with jets whilst requiring a highly boosted $Z / \gamma^{*}$ boson, we have selected a difficult phase space to model, sensitive to soft hadronic activity and jet shapes. It is important to model $p_{\mathrm{T}, \ell \ell}$ accurately, as other observables such as $\Delta \phi(\ell, \ell)$ and $p_{\mathrm{T}, \ell}^{\text {lead }}$ are correlated.

For this reason, a data-driven correction to the $p_{\mathrm{T}, Z}$ distribution is employed. It is derived by comparing the observed and predicted $p_{\mathrm{T}, \ell \ell}$ distributions in 0 -jet $\mu \mu$ events with $\left|m_{\ell \ell}-m_{Z}\right|<15 \mathrm{GeV}$. The $p_{\mathrm{T}, Z}$ distribution in $\mathrm{MC}$ is then multiplied by this $p_{\mathrm{T}, \ell \ell}$ correction. This is found to improve the modelling of detector-level observables such as $p_{\mathrm{T}, \ell \ell}, \Delta \phi(\ell, \ell)$ and $p_{\mathrm{T}, \ell}^{\text {lead }}$ (see Figure 7.10). This correction is applied to the $Z / \gamma^{*} \rightarrow e e / \mu \mu / \tau \tau$ processes, though only in the 0 -jet bin.

\footnotetext{
${ }^{5}$ In rare cases, $Z / \gamma^{*} \rightarrow \ell \ell$ events can enter the $e \mu / \mu e$ channels. For example $Z / \gamma^{*} \rightarrow \mu \mu \gamma$, where a muon radiates a photon which subsequently converts and is reconstructed as an electron.
} 

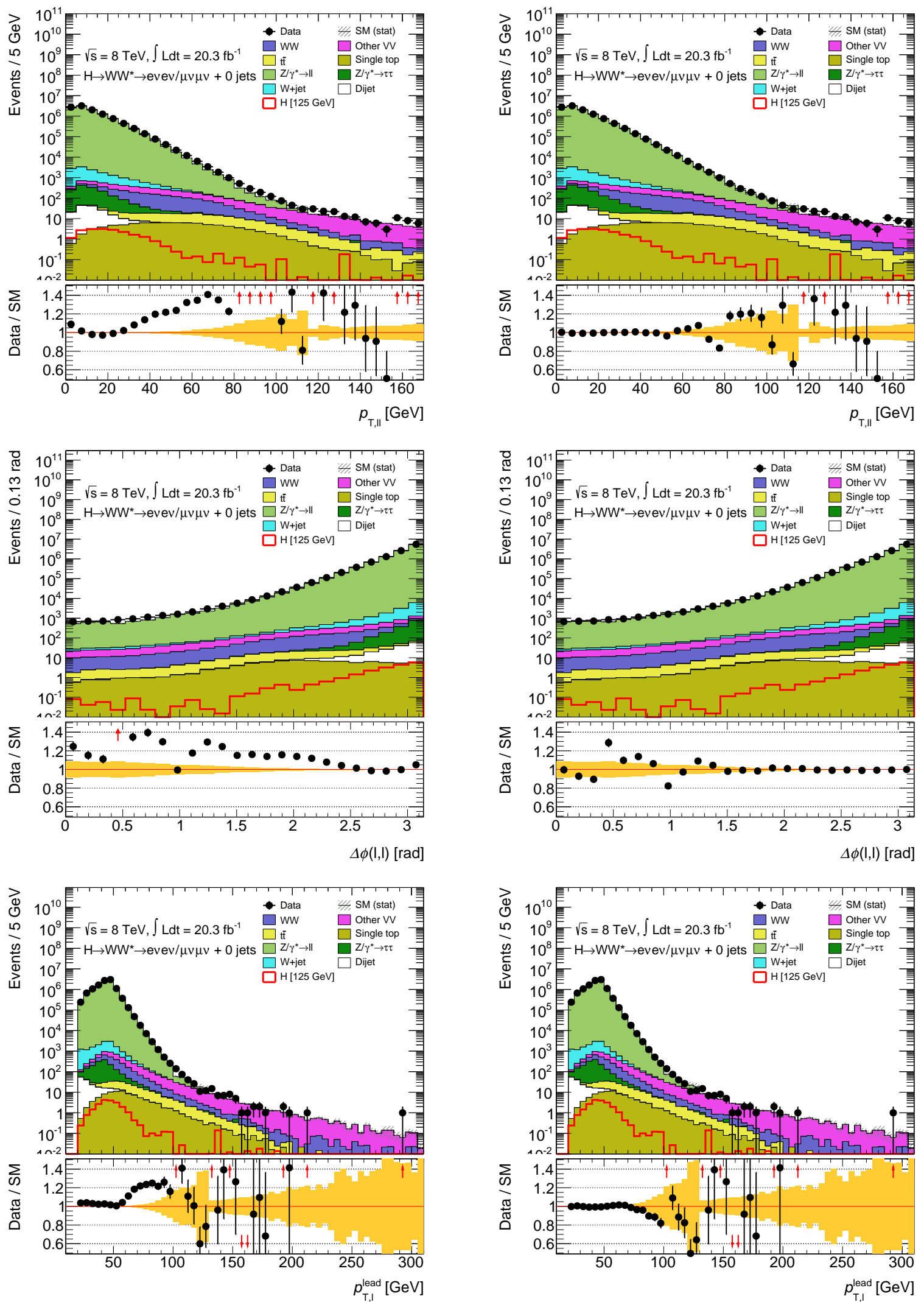

Figure 7.10: Leptonic distributions in the 0 -jet $Z / \gamma^{*} \rightarrow \ell \ell$ control region, before (left) and after (right) the $p_{\mathrm{T}, Z}$ correction is applied. 


\begin{tabular}{|c|c|c|}
\hline \multicolumn{3}{|c|}{$e \mu / \mu e$} \\
\hline \multicolumn{3}{|c|}{$\begin{array}{c}p_{\mathrm{T}, \ell}^{\text {lead }}>22 \text { and } p_{\mathrm{T}, \ell}^{\text {sublead }}>10 \\
m_{\ell \ell}>12 \\
p_{\mathrm{T}}^{\text {miss,corr }}>20 \\
\end{array}$} \\
\hline 0-jet bin & 1-jet bin & $\geq 2$-jet bin \\
\hline- & $N_{b \text {-jets }}=0$ & $N_{b \text {-jets }}=0$ \\
\hline- & $\max \left(m_{\mathrm{T}, \ell}\right)>50$ & - \\
\hline- & $m_{\tau \tau}>m_{Z}-25$ & - \\
\hline- & - & Fail CJV or OLV \\
\hline$m_{\ell \ell}<80$ & $m_{\ell \ell}<80$ & $m_{\ell \ell}<70$ \\
\hline$\Delta \phi(\ell, \ell)>2.8$ & - & $\Delta \phi(\ell, \ell)>2.8$ \\
\hline
\end{tabular}

Table 7.2: Event selection criteria of the $Z / \gamma^{*} \rightarrow \tau \tau$ control regions. Cuts on energy, momentum and mass are given in $\mathrm{GeV}$, and angular cuts are given in radians. The relevant observables are described in Chapter 4.

The $p_{\mathrm{T}, Z}$ mismodelling might be different in the signal region to the $Z$ control region where it is derived. The validity of this extrapolation of the correction was tested by deriving similar corrections to a SHERPA sample (instead of to experimental data). This indicated there is a correlation between the correction and the $p_{\mathrm{T}}^{\text {inv }}$ requirement. Thus, another data-driven correction is derived with an additional $p_{\mathrm{T}}^{\text {miss,corr }}>20 \mathrm{GeV}$ cut, which is used to estimate the uncertainty in the correction.

\subsubsection{Z/ $\gamma^{*} \rightarrow \tau \tau$ estimation}

The $Z / \gamma^{*} \rightarrow \tau \tau$ background is measured in a dedicated control region (CR), and then extrapolated to the signal region (SR) using $\mathrm{MC}$

$$
\begin{aligned}
N_{Z \rightarrow \tau \tau}^{\mathrm{pred}, \mathrm{SR}} & =\alpha_{Z \rightarrow \tau \tau} \cdot\left(N^{\text {data,CR }}-N_{\text {non- } Z \rightarrow \tau \tau}^{\mathrm{pred}, \mathrm{CR}}\right) \\
\alpha_{Z \rightarrow \tau \tau} & =N_{Z \rightarrow \tau \tau}^{\mathrm{MC}, \mathrm{SR}} / N_{Z \rightarrow \tau \tau}^{\mathrm{MC}, \mathrm{CR}} .
\end{aligned}
$$

CRs are defined in each jet bin of the $e \mu+\mu e$ channel, according to the event selection criteria in Table 7.2. Each $e \mu+\mu e$ CR is used to extrapolate to the corresponding 

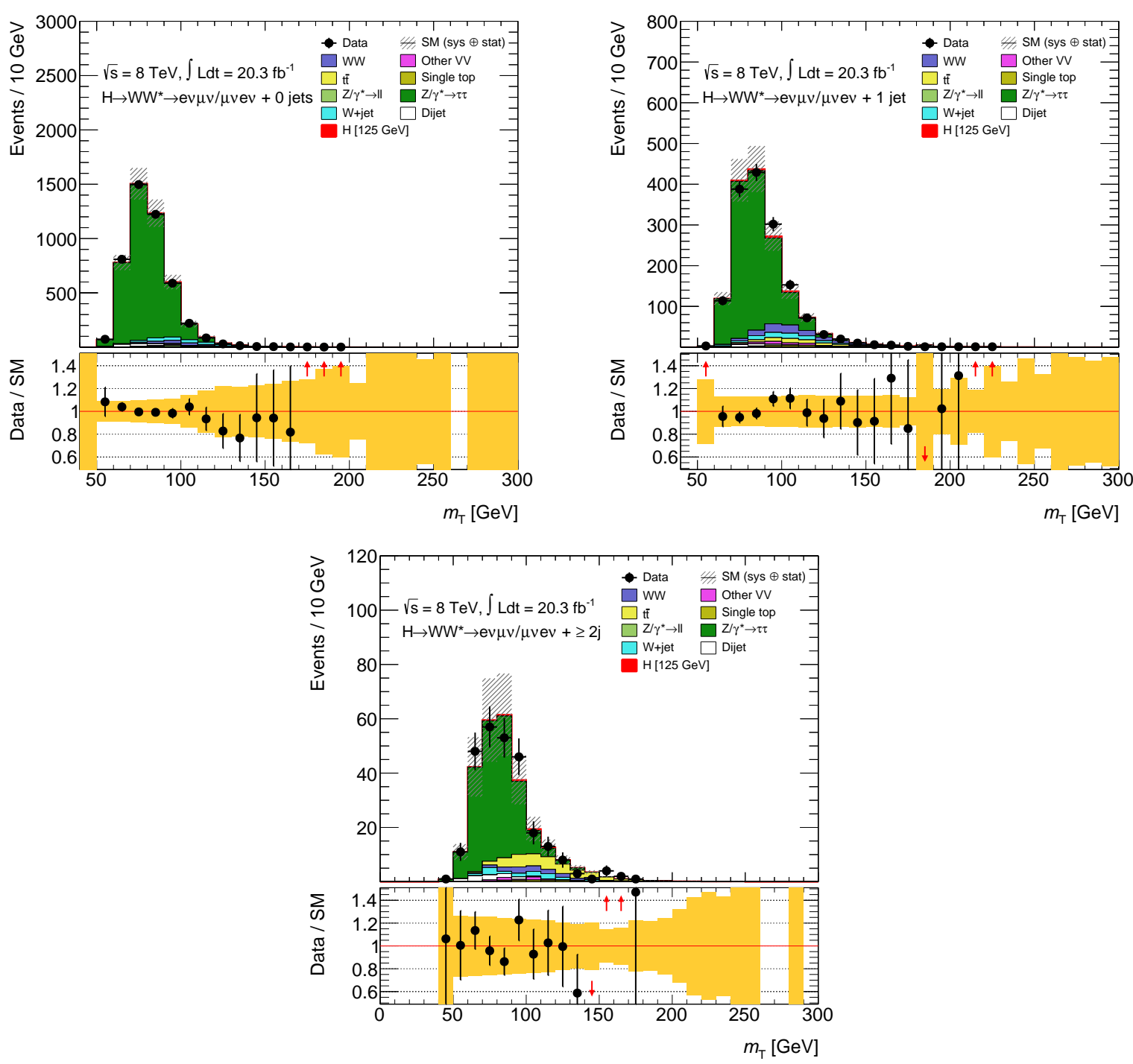

Figure 7.11: The $m_{\mathrm{T}}$ distribution in the $Z / \gamma^{*} \rightarrow \tau \tau$ control regions of the 0 -jet (left), 1-jet (right) and $\geq 2$-jet (bottom) bins. Normalisation factors are applied.

jet-binned $e \mu / \mu e / e e+\mu \mu$ SRs. Figure 7.11 exhibits the excellent MC modelling of the shapes of this background, following application of the normalisation factors. 


\subsubsection{Z $/ \gamma^{*} \rightarrow \ell \ell$ estimation}

$Z / \gamma^{*} \rightarrow \ell \ell$ is the dominant background to the $e e / \mu \mu$ channels, and this section will describe how it is estimated in the corresponding 0 -jet and 1 -jet bins (the $\geq 2$-jet bin is not used in the $e e / \mu \mu$ channels).

$Z / \gamma^{*} \rightarrow \ell \ell$ is largely rejected by the $Z$ boson mass veto, $\left|m_{\ell \ell}-m_{Z}\right|>15 \mathrm{GeV}$, and the requirement of large missing transverse momentum, $E_{\mathrm{T}, \mathrm{rel}}^{\mathrm{miss}}>40$ (40) GeV and $p_{\mathrm{T}, \text { rel }}^{\text {miss }}>40(35) \mathrm{GeV}$ in the 0-jet (1-jet) bin. Although the $p_{\mathrm{T}}^{\text {inv }}$ resolution is broadened by pile-up, for events to possess such high $E_{\mathrm{T} \text {,rel }}^{\text {miss }}$ and $p_{\mathrm{T}, \text { rel }}^{\text {miss }}$ suggests there is some mismeasured hadronic activity recoiling against the dilepton $(+$ jet $)$ system. This situation is also enhanced by the $p_{\mathrm{T}, \ell \ell}>30 \mathrm{GeV}$ cut in the 0 -jet bin.

This hadronic recoil must be soft and broad in order to avoid passing the jet reconstruction, and might not be modelled accurately. It is therefore preferable to use a data-driven estimation of this background. Fortunately, the $f_{\text {recoil }}$ observable (described below) can discriminate $Z / \gamma^{*} \rightarrow \ell \ell$ from other processes in this phase space, and can be used to simultaneously suppress and estimate the $Z / \gamma^{*} \rightarrow \ell \ell$ background.

First, soft jets with $p_{\mathrm{T}}>10 \mathrm{GeV}$ and $|\eta|<4.5$ are found, following the jet selection detailed in Section 4.2.5 minus the JVF cut. In the 0 -jet bin, $f_{\text {recoil }}$ is defined by

$$
f_{\text {recoil }}=\left|\sum_{j \text { in } \wedge} \mathrm{JVF}_{j} \cdot \boldsymbol{p}_{\mathrm{T}, j}\right| / p_{\mathrm{T}, \ell \ell}
$$

where $\wedge$ is the detector quadrant centred on $-\boldsymbol{p}_{\mathrm{T}, \ell \ell}$. Approximately speaking, this is the fraction of $p_{\mathrm{T}, \ell \ell}$ that can be balanced by soft hadronic activity in the opposing quadrant. In the 1-jet bin, it is the dilepton + jet system that must be balanced, and thus the quadrant $\wedge$ is centred upon $-\boldsymbol{p}_{\mathrm{T}, \ell \ell j}$ and the denominator becomes $p_{\mathrm{T}, \ell \ell j}$.

The tight $p_{\mathrm{T}}^{\text {inv }}$ criteria sculpt $f_{\text {recoil }}$ in $Z / \gamma^{*} \rightarrow \ell \ell$ towards higher values, whereas the other background processes (and signal) feature high- $p_{\mathrm{T}}$ neutrinos and peak at lower values (see Figure 7.12). A template method exploits the markedly different $f_{\text {recoil }}$ shape of the $Z / \gamma^{*} \rightarrow \ell \ell$ background to estimate its contribution to the $e e+\mu \mu$ signal region.

In the template method, the signal region (SR) is defined by the full event selection of the $e e / \mu \mu$ channels, excluding the $f_{\text {recoil }}$ cut (whose efficiency shall be predicted by the method). The $f_{\text {recoil }}$ distribution $\mathcal{T}$ in the $\mathrm{SR}$ of the same-flavour (SF) channels, i.e. the $e e / \mu \mu$ channels, has two components with distinct shapes: $Z / \gamma^{*} \rightarrow \ell \ell$, and other 

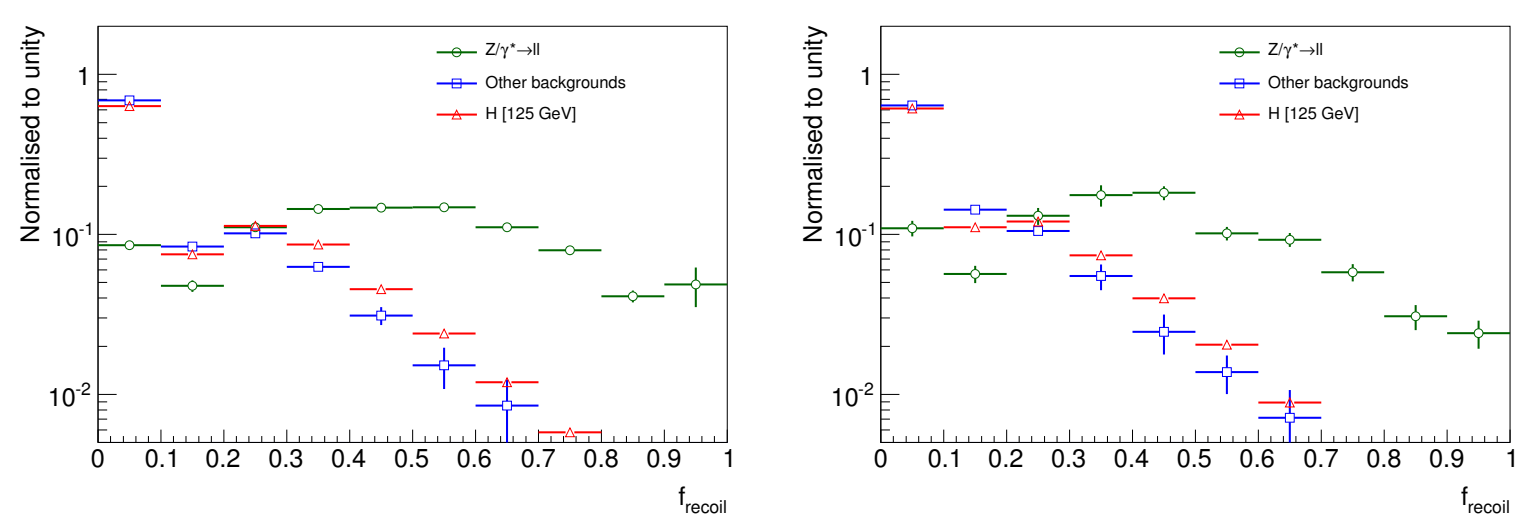

Figure 7.12: $f_{\text {recoil }}$ shape in the 0 -jet (left) and 1-jet (right) signal regions of the $e e / \mu \mu$ channels, excluding the $f_{\text {recoil }}$ cut itself. The $W+$ jet and dijet backgrounds are data-driven and all other processes are described by MC. Error bars show statistical uncertainties.

processes (including signal). If the shape of both components are predicted, then their relative contributions can be fit using the observed $f_{\text {recoil }}$ distribution in the SR

$$
\mathcal{T}^{\text {data,SR,SF }}=K_{\text {non- } Z \rightarrow \ell \ell}^{\mathrm{fit}} \cdot \mathcal{T}_{\text {non- } Z \rightarrow \ell \ell}^{\mathrm{pred}, \mathrm{SR}, \mathrm{SF}}+K_{Z \rightarrow \ell \ell}^{\mathrm{fit}} \cdot \mathcal{T}_{Z \rightarrow \ell \ell}^{\mathrm{pred}, \mathrm{SR}, \mathrm{SF}}
$$

where $K_{i}^{\text {fit }}$ are prefactors determined by the fitting procedure, and the input distributions $\mathcal{T}_{i}^{\text {pred,SR,SF }}$ are determined by data-driven methods described below.

$\mathcal{T}_{\text {non- } Z \rightarrow \ell \ell}^{\text {pred,SR }}$ is determined from the observed distribution in different-flavour (DF) events, i.e. the $e \mu / \mu$ e channels, $\mathcal{T}^{\text {data,SR,DF }}$. Note that this DF distribution is measured in the SR defined by the SF event selection criteria. In doing this, the normalisation factor $K_{\text {non- } Z \rightarrow \ell \ell}^{\mathrm{fit}}$ becomes an extrapolation parameter $\alpha_{\text {non- } Z \rightarrow \ell \ell}^{\mathrm{fit}}$ from DF to SF. This method is valid because the $Z / \gamma^{*} \rightarrow \ell \ell$ background to the DF channels is negligible, and the composition of the other processes is consistent between DF and SF events.

$\mathcal{T}_{Z \rightarrow \ell \ell}^{\text {pred,SR,SF }}$ is determined from the SF distribution measured in a $Z / \gamma^{*} \rightarrow \ell \ell$ control region $(\mathrm{CR}) \mathcal{T}^{\text {data, } \mathrm{CR}, \mathrm{SF}}$. The $\mathrm{CR}$ is defined by the same selection criteria as the $\mathrm{SR}$, except that the $m_{\ell \ell}<55 \mathrm{GeV}$ criteria becomes $\left|m_{\ell \ell}-m_{Z}\right|<15 \mathrm{GeV}$. As the $p_{\mathrm{T}}^{\mathrm{inv}}$ and $p_{\mathrm{T}, \ell \ell}$ criteria remain in the $\mathrm{CR}$ definition, $f_{\text {recoil }}$ is sculpted similarly to the SR. However, this also means that the contribution of other processes in the CR is non-negligible and must be subtracted. This contamination is estimated from the distribution measured in $\mathrm{DF}$ events passing the same $\mathrm{CR}$ selection $\mathcal{T}^{\text {data, } \mathrm{CR}, \mathrm{DF}}$, with the extrapolation from $\mathrm{DF}$ to SF predicted by dedicated data-driven methods described elsewhere or by MC. 
Thus, (7.24) becomes

$$
\mathcal{T}^{\text {data,SR,SF }}=\alpha_{\text {non- } Z \rightarrow \ell \ell}^{\text {fit }} \cdot \mathcal{T}^{\text {data,SR,DF }}+\alpha_{Z \rightarrow \ell \ell}^{\text {fit }} \cdot\left(\mathcal{T}^{\text {data, } \mathrm{CR}, \mathrm{SF}}-\alpha_{\text {non- } Z \rightarrow \ell \ell}^{\text {pred }} \cdot \mathcal{T}^{\text {data, } \mathrm{CR}, \mathrm{DF}}\right)
$$

where $\alpha_{\text {non- } Z \rightarrow \ell \ell}^{\mathrm{fit}}$ and $\alpha_{\text {non- } Z \rightarrow \ell \ell}^{\text {pred }}$ are extrapolations from DF to SF, and $\alpha_{Z \rightarrow \ell \ell}^{\mathrm{fit}}$ is an extrapolation in $m_{\ell \ell}$. Note that the $\alpha_{i}$ are prefactors and leave template shapes unchanged. The sensitivity of this method to the signal strength is tested and found to be negligible.

The final event selection criterion in the $e e / \mu \mu$ channels is $f_{\text {recoil }}<0.1$. The efficiency of this cut for both $Z / \gamma^{*} \rightarrow \ell \ell$ and other processes is inherently predicted by the template method. To simplify matters, the templates are reduced to two bins: $f_{\text {recoil }}<0.1$ and $f_{\text {recoil }}>0.1$. With two bins and two free parameters $\alpha_{i}^{\text {fit }}$, the system can be solved exactly: instead of fitting the $f_{\text {recoil }}$ shape, the method estimates the efficiency of the $f_{\text {recoil }}$ cut. The dominant uncertainty in the 0 -jet $Z / \gamma^{*} \rightarrow \ell \ell$ estimation arises from correlations between $f_{\text {recoil }}$ and $m_{\ell \ell}$, and is evaluated with MC to be $32 \%$. The 1 -jet bin is dominated by statistical uncertainties.

\subsection{Summary of normalisation factors}

It is helpful to express the data-driven estimations of several backgrounds in terms of a normalisation factor, which is the ratio of the data-driven predicted yield to the MC-only predicted yield. Their pre-fit and post-fit values are summarised in Table 7.3, where the fit can change the values due to the pull of nuisance parameters (see Section 8.2.2).

These can also be used to improve the background estimations in regions other than the signal region; however, this does neglect theoretical uncertainties in the extrapolation from the corresponding control region. Plotted distributions in Chapters 4-7 use pre-fit normalisation factors in this way. 


\begin{tabular}{lccc}
\hline \multirow{2}{*}{ Process } & \multicolumn{3}{c}{ Pre-fit (post-fit) normalisation factor } \\
& 0-jet & 1-jet & $\geq 2$-jet \\
\hline$W W$ & $1.22(1.22)$ & $1.06(1.12)$ & - \\
Non- $W W$ diboson & $0.91(0.95)$ & $0.95(0.84)$ & - \\
Top & $1.09(1.09)$ & $1.04(1.03)$ & $1.00(1.00)$ \\
$Z / \gamma^{*} \rightarrow \tau \tau$ & $1.00(0.99)$ & $1.05(1.05)$ & $0.96(0.97)$ \\
\hline
\end{tabular}

Table 7.3: The data-driven normalisation factor used to scale the MC description of each background process. These can change in the fit due to the pull of nuisance parameters (see Section 8.2.2). The $W+$ jet and dijet processes are fully datadriven. 


\section{Chapter 8}

\section{Experimental results}

Chapters 4-7 describe the details of the $H \rightarrow W W$ analysis: the selection of signal events and the rejection of backgrounds, and how each process is modelled. By comparing the expected and observed events passing the selection criteria, with a careful treatment of statistical and systematic uncertainties, it is possible to make statistically meaningful statements about the $H \rightarrow W W$ process, based upon the Run I dataset.

Section 8.1 briefly summarises the experimental and theoretical sources of systematic uncertainty. Then, Section 8.2 describes the statistical treatment used to extract the experimental results, which are finally presented in Section 8.3.

\subsection{Systematic uncertainties}

In addition to statistical uncertainties in the observed number of events and the expected number of events (due to finite MC sample sizes), multiple sources of systematic uncertainty should be considered. Many of these were introduced throughout Chapters 4-7, but a short summary is presented here.

\subsubsection{Experimental uncertainties}

Experimental uncertainties arise due to a mismodelling of the detector performance, particularly in the reconstruction efficiency and energy calibration of physics objects. These introduce uncertainties either directly into the expected acceptance (as with signal) or via the extrapolation from a control region (as with many backgrounds). 


\section{Trigger efficiency}

The lepton trigger efficiencies and their uncertainties are measured as a function of $p_{\mathrm{T}}$ and $\eta$ using tag-and-probe of $Z \rightarrow \ell \ell$ events (see Section 4.3.2). This includes the efficiency of matching the trigger to the lepton object.

\section{Lepton reconstruction efficiency}

The lepton selection efficiencies and their uncertainties are measured as a function of $p_{\mathrm{T}}$ and $\eta$ using tag-and-probe of $Z \rightarrow \ell \ell$ events (see Sections 4.2.3 and 4.2.4). This is performed separately for each step in the lepton selection, i.e. reconstruction, identification, isolation and primary vertex association. The efficiency uncertainties are $<0.5 \%$ for muons and $<3 \%$ for electrons.

\section{Lepton energy scale and resolution}

Lepton energy scales and resolutions are calibrated in situ as a function of $p_{\mathrm{T}}$ and $\eta$ using $J / \psi \rightarrow \ell \ell$ and $Z \rightarrow \ell \ell$ resonance events, and the associated uncertainties are also derived during this calibration. Scale uncertainties are $<0.5 \%$ and their impact is assessed by varying lepton energies by $\pm 1 \sigma$. Resolution uncertainties are $<1 \%$ and their impact is assessed by varying the smearing of lepton energies by $\pm 1 \sigma$.

\section{Jet reconstruction efficiency}

The jet selection criteria feature a cut upon the jet vertex fraction (JVF) in order to reduce pile-up jets (see Section 4.2.5). Unlike the trigger, lepton reconstruction and $b$-tagging efficiencies, the JVF efficiency is not subject to a data-driven scale factor and so MC mismodelling introduces a systematic uncertainty to the $N_{\text {jets }}$ distribution. The mismodelling of the JVF efficiency is evaluated as $1.2 \%$ in a $Z / \gamma^{*} \rightarrow \ell \ell$ control region, resulting in negligible jet bin migrations. The data-driven background estimations are jet-binned and mostly avoid this issue.

\section{Jet energy scale and resolution}

The jet energy scale (JES) is calibrated as a function of $p_{\mathrm{T}}$ and $\eta$, as described in Section 4.2.5. The in situ calibration method of balancing jets against well-measured reference objects introduces experimental and theoretical uncertainties to the JES. Also, uncertainties in the pile-up environment introduce uncertainties to the JES through the pile-up subtraction step of the calibration. Finally, the JES is affected by uncertainties in the jet flavour composition and the detector response to each flavour. The uncertainty components are treated separately (the total uncertainty is $<7 \%$ ) and their impact is assessed by varying jet energies by $\pm 1 \sigma$. 
The jet energy resolution (JER) and its uncertainty are measured in situ as a function of $p_{\mathrm{T}}$ and $\eta$ using dijet events [136]. The bisector method projects the dijet $p_{\mathrm{T}}$ imbalance in the direction bisecting the two jets and in the orthogonal direction. At hadron-level the two components are expected to have the same variance, but at detector-level the variance of the latter is increased by the JER. Thus, measurements of the two components allow the JER to be evaluated. The uncertainty is $<10 \%$ and its impact is assessed by increasing the smearing of jet energies by $+1 \sigma$.

\section{Jet b-tagging efficiency}

The $b$-tagging efficiency for $b$-jets is calibrated as a function of jet $p_{\mathrm{T}}$, using dileptonic $t \bar{t}$ decays in a combinational likelihood method [116]. At $p_{\mathrm{T}} \approx 20 \mathrm{GeV}$ the measurement is limited by JES uncertainties, but at $p_{\mathrm{T}}>40 \mathrm{GeV}$ it is limited by modelling uncertainties in the jet flavour composition. Uncertainties in the $b$-tagging efficiency of $c$-jets and light jets are also considered, though have little effect.

\section{$p_{\mathrm{T}}^{\text {inv }}$ modelling}

As described in Section 4.2.7, $E_{\mathrm{T}}^{\text {miss }}, p_{\mathrm{T}}^{\text {miss }}$ and $p_{\mathrm{T}}^{\text {miss,corr }}$ are defined using the calibrated electron, muon and jet objects, and are therefore correlated to the electron, muon and jet energy scales whose uncertainties are outlined above. In the $E_{\mathrm{T}}^{\mathrm{miss}}$ calculation, uncertainties in the scale of the unassociated soft calorimeter deposits are considered. In the $p_{\mathrm{T}}^{\text {miss }}$ and $p_{\mathrm{T}}^{\text {miss,corr }}$ calculations, uncertainties in the $\boldsymbol{p}_{\mathrm{T}}$ imbalance of tracks unassociated with a physics object are considered (e.g. due to the presence of neutral particles).

\section{Lepton fake factors}

The lepton fake factors $f_{\ell}$ are important to the $W+$ jet and dijet background estimations, and are detailed in Section 7.1. $f_{\ell}^{W+\text { jet }}$ is measured in $Z+$ jet events, and is dominated by statistical uncertainties, uncertainties in the electroweak background subtraction and theoretical uncertainties in MC-based corrections. However, a component of the uncertainties cancels in the same-sign control region method (see Section 7.2.1). $f_{\ell}^{\text {dijet }}$ is measured in dijet events and is dominated by theoretical uncertainties in MC-based corrections.

\section{Photon conversions}

Modelling photon conversions is important to the $W \gamma$ background estimation, in particular the efficiency of two electron identification criteria: the hit in the first pixel layer and the conversion vertex reconstruction. Mismodelling of these criteria is tested in $Z \gamma$ events and applied as a systematic uncertainty (see Section 7.2.2). 


\section{Pile-up}

Uncertainties in the pile-up environment affect the jet calibration, as outlined above. Pile-up can also introduce additional hard jets that lead to migrations between jet bins. Uncertainties in pile-up lead to migration uncertainties of $0.5 \%$ and $1.0 \%$ in the 0 -jet and 1-jet bins respectively, and are neglected as they are much smaller than the ggF jet binning uncertainties.

The effect of out-of-time pile-up upon detector electronics might also be mismodelled. This is found to be negligible.

\section{Luminosity}

The luminosity measurement is calibrated during van der Meer scans (see Section 3.2.1), with a relative uncertainty of $2.8 \%$ for the $\sqrt{s}=8 \mathrm{TeV}$ dataset.

\subsubsection{Theoretical uncertainties}

Many theoretical uncertainties are also considered (and indeed are included within the experimental uncertainties above). Theoretical uncertainties in the expected signal are described in Chapter 5. Theoretical uncertainties in the expected background appear when some aspect of a data-driven technique relies upon MC modelling. Some of the uncertainties considered are presented in Chapters 6 and 7.

The sources of uncertainty usually considered are: higher order corrections in the perturbative series, incoming parton distribution functions, and aspects of the MC modelling (e.g. hadronisation and underlying event models).

\subsection{Statistical model}

A statistical model is built within the HistFACTORY software package [144], incorporating the various data-driven techniques described in Chapters 6 and 7 . The experimental data and MC-modelled expectations found within each signal and control region allow the model to be constrained, and for various hypotheses to be tested. 


\begin{tabular}{|c|c|c|c|c|c|}
\hline \multirow{2}{*}{$N_{\text {jets }}$} & \multirow{2}{*}{ Channel } & \multicolumn{3}{|c|}{ Bin boundaries $(\mathrm{GeV})$} & \multirow{2}{*}{$N_{\text {bin }}$} \\
\hline & & $p_{\mathrm{T}, \ell}^{\text {sublead }}$ & $m_{\ell \ell}$ & $m_{\mathrm{T}}$ & \\
\hline 0 -jet & $e \mu, \mu e$ & {$[10,15,20, \infty]$} & {$[10,30,55]$} & 10 bins & 60 \\
\hline 0 -jet & $e e+\mu \mu$ & {$[10, \infty]$} & {$[12,55]$} & 10 bins & 10 \\
\hline 1-jet & $e \mu, \mu e$ & {$[10,15,20, \infty]$} & {$[10,30,55]$} & 6 bins & 36 \\
\hline 1-jet & $e e+\mu \mu$ & {$[10, \infty]$} & {$[12,55]$} & 6 bins & 6 \\
\hline$\geq 2$-jet & $e \mu, \mu e$ & {$[10, \infty]$} & {$[10,55]$} & {$[0,50,80,130, \infty]$} & 4 \\
\hline
\end{tabular}

Table 8.1: The binning of each discriminant observable for each signal region. The $m_{\mathrm{T}}$ bin boundaries in the 0 -jet and 1 -jet bins are chosen to produce a flat signal.

\subsubsection{Discriminant observables}

The fitting procedure discriminates between the signal and background processes through the distribution of certain sensitive observables. However, the degree to which this information can be exploited is limited by statistical uncertainties. The sensitivity of the fitting procedure can be optimised through the choice of discriminating observables, the number of bins in each observable, and the location of the bin boundaries.

The analysis features eight signal regions, defined by the criteria in Section 4.3: $\{e \mu, \mu e, e e+\mu \mu\} \otimes\{0$-jet,1-jet $\} \oplus\{e \mu, \mu e\} \otimes\{\geq 2$-jet $\}$. A three-dimensional fit of $m_{\mathrm{T}}, p_{\mathrm{T}, \ell}^{\text {sublead }}$ and $m_{\ell \ell}$ is performed in the $\{e \mu, \mu e\} \otimes\{0$-jet,1-jet $\}$ signal regions, whereas a one-dimensional $m_{\mathrm{T}}$ fit is used in the others.

Additionally, in the $\{e \mu, \mu e, e e+\mu \mu\} \otimes\{0$-jet,1-jet $\}$ signal regions, the $m_{\mathrm{T}}$ bin boundaries are chosen such that there are an equal number of expected signal events in each bin. This is applied independently within each $p_{\mathrm{T}, \ell}^{\text {sublead }} m_{\ell \ell}$ bin in the $e \mu / \mu e$ channels. In the $\geq 2$-jet bin, fixed $m_{\mathrm{T}}$ bin boundaries are used.

The binning of each discriminant observable is shown in Table 8.1, and the inclusive $m_{\mathrm{T}}$ distribution of each jet bin is shown in Figure 8.1. 

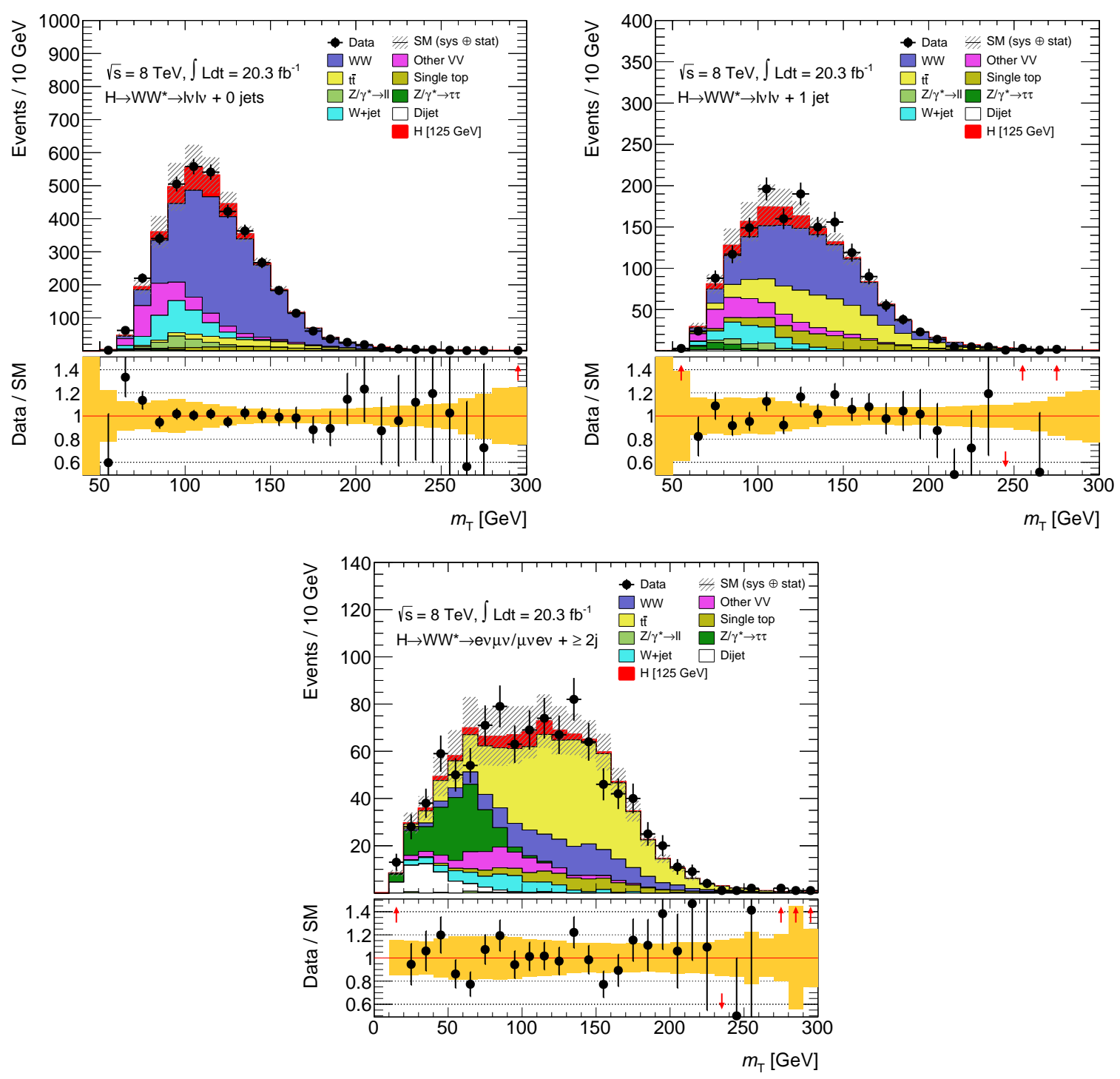

Figure 8.1: The $m_{\mathrm{T}}$ distribution in the signal region of the 0-jet (left), 1-jet (right) and $\geq 2$-jet (bottom) bin. Normalisation factors are applied. 


\subsubsection{Likelihood function}

The statistical model describing the experiment depends upon a set of parameters $\boldsymbol{\alpha}=(\mu, \boldsymbol{\theta})$, where $\mu=\sigma / \sigma_{\mathrm{SM}}$ is the signal strength (a parameter of interest) and $\boldsymbol{\theta}$ is the set of nuisance parameters (e.g. trigger efficiency, $W W$ extrapolation parameter $\left.\alpha_{W W}\right)$. The Higgs boson mass $m_{H}$ could also be treated as a parameter of interest, but hypothesis testing is usually performed as a raster scan of $m_{H}$ in practice.

The likelihood function expresses how likely a set of parameter values are, given that a particular dataset is observed. It is defined as the probability of producing the observed dataset, when the parameter values are input into the statistical model,

$$
\begin{aligned}
\mathcal{L}(\mu, \boldsymbol{\theta}) & =\mathcal{L}\left(\mu, \boldsymbol{\theta} \mid \mathcal{D}^{\mathrm{SR}}, \mathcal{D}^{\mathrm{CR}}\right) \\
& =f\left(\mathcal{D}^{\mathrm{SR}}, \mathcal{D}^{\mathrm{CR}} ; \mu, \boldsymbol{\theta}\right)
\end{aligned}
$$

where $\mathcal{D}^{\mathrm{SR}}$ and $\mathcal{D}^{\mathrm{CR}}$ are the observed datasets in the signal regions (SRs) and control regions (CRs), respectively. This can be decomposed into a product of probabilities

$$
\mathcal{L}(\mu, \boldsymbol{\theta})=\prod_{c \in \text { channels }} f\left(\mathcal{D}_{c}^{\mathrm{SR}} ; \mu, \boldsymbol{\theta}\right) \prod_{c \in \text { channels }} f\left(\mathcal{D}_{c}^{\mathrm{CR}} ; \mu, \boldsymbol{\theta}\right) \prod_{i \in \text { n.p. }} f_{i}\left(\tilde{\theta}_{i} ; \theta_{i}, \Delta \tilde{\theta}_{i}\right)
$$

where the SR channels are the eight SRs described in Section 8.2.1, and the CR channels include the data used in data-driven background estimations. Each $f_{i}\left(\tilde{\theta}_{i} ; \theta_{i}, \Delta \tilde{\theta}_{i}\right)$ acts to constrain the corresponding nuisance parameter, and is often a Gaussian distribution. The nominal value $\tilde{\theta}$ and uncertainty $\Delta \tilde{\theta}$ are determined by an auxiliary measurement, either experimental (e.g. trigger efficiency) or theoretical (e.g. $\alpha_{W W}$ ).

The probability of producing an observed distribution $\mathcal{D}_{c}$ is described by a product of Poisson distributions

$$
f\left(\mathcal{D}_{c} ; \mu, \boldsymbol{\theta}\right)=\prod_{b \in \text { bins }} \operatorname{Pois}\left(N_{c b}^{\mathrm{obs}} ; \mu N_{c b}^{\mathrm{sig}}(\boldsymbol{\theta})+N_{c b}^{\mathrm{bkg}}(\boldsymbol{\theta})\right)
$$

where $\operatorname{Pois}(x ; \lambda)=\lambda^{x} e^{-\lambda} / x !, N_{c b}^{\text {obs }}$ is the number of observed events, $N_{c b}^{\text {sig }}(\boldsymbol{\theta})$ is the number of expected signal events for a Standard Model Higgs boson (estimated by $\mathrm{MC}$ ), and $N_{c b}^{\mathrm{bkg}}(\boldsymbol{\theta})$ is the number of expected background events (estimated by datadriven techniques embedded in the statistical model). The product is over the bins of the distribution $\mathcal{D}_{c}$, which is one- or three-dimensional in the case of the SRs, and zero-dimensional in many of the CRs (i.e. a single bin). 
The expected numbers of events depend upon the nuisance parameters according to

$$
\begin{aligned}
N_{c b}^{\mathrm{sig}}(\boldsymbol{\theta}) & =N_{c b}^{\mathrm{sig}}(\tilde{\boldsymbol{\theta}}) \prod_{i \in \text { n.p. }} \nu_{c b i}\left(\theta_{i}-\tilde{\theta}_{i}\right) \\
N_{c b}^{\mathrm{bkg}}(\boldsymbol{\theta}) & =\sum_{p \in \text { processes }} N_{c b p}^{\mathrm{bkg}}(\tilde{\boldsymbol{\theta}}) \prod_{i \in \text { n.p. }} \nu_{c b p i}\left(\theta_{i}-\tilde{\theta}_{i}\right)
\end{aligned}
$$

where $N(\tilde{\boldsymbol{\theta}})$ is the expected events with the nominal nuisance parameters as determined by auxiliary measurements, and $\nu_{i}\left(\theta_{i}-\tilde{\theta}_{i}\right)$ factorises the dependence upon the nuisance parameter $\theta_{i}$. The $\nu_{i}\left(\theta_{i}-\tilde{\theta}_{i}\right)$ are often determined by evaluating $\nu_{i}\left(+\Delta \tilde{\theta}_{i}\right)$ and $\nu_{i}\left(-\Delta \tilde{\theta}_{i}\right)$, and then interpolating and extrapolating. Some nuisance parameters affect all processes (e.g. trigger efficiency), whilst others only affect a particular process (e.g. $\left.\alpha_{W W}\right)$. Correlations can exist between bins and channels, e.g. the $\nu_{i}\left(\theta_{i}-\tilde{\theta}_{i}\right)$ for a normalisation parameter would be $100 \%$ correlated between bins. Statistical uncertainties in the MC samples are implemented as uncorrelated uncertainties in the total expected events in each bin, where the corresponding constraint $f_{i}\left(\tilde{\theta}_{i} ; \theta_{i}, \Delta \tilde{\theta}_{i}\right)$ is a Poisson distribution.

\subsubsection{Hypothesis testing}

The statistical techniques of hypothesis testing employed by Higgs boson searches at the LHC are fully described in references $[145,146]$. Those used in the $H \rightarrow W W$ search to exclude, discover and measure the Higgs boson are briefly summarised below.

Hypothesis testing is based around the profile likelihood ratio, defined as

$$
\lambda(\mu)=\frac{\mathcal{L}(\mu, \hat{\hat{\boldsymbol{\theta}}}(\mu))}{\mathcal{L}(\hat{\mu}, \hat{\boldsymbol{\theta}})}
$$

where $\hat{\mu}$ and $\hat{\boldsymbol{\theta}}$ are the parameter values that maximise the likelihood, and $\hat{\hat{\boldsymbol{\theta}}}(\mu)$ are the nuisance parameter values that maximise the likelihood when $\mu$ is fixed. Maximal agreement with experimental data is expressed by the maximum $\lambda(\hat{\mu})=1$, and statistical and systematic uncertainties determine the shape of $\lambda(\mu)$. The maximised $\hat{\boldsymbol{\theta}}$ and $\hat{\hat{\boldsymbol{\theta}}}(\mu)$ can be pulled, and constrained differently, with respect to the auxiliary measurements $\tilde{\boldsymbol{\theta}}$. 
An alternative version of the profile likelihood ratio is defined as

$$
\tilde{\lambda}(\mu)= \begin{cases}\frac{\mathcal{L}(\mu, \hat{\boldsymbol{\theta}}(\mu))}{\mathcal{L}(\hat{\mu}, \hat{\boldsymbol{\theta}})} & \text { if } \hat{\mu} \geq 0 \\ \frac{\mathcal{L}(\mu, \hat{\boldsymbol{\theta}}(\mu))}{\mathcal{L}(0, \hat{\hat{\boldsymbol{\theta}}}(0))} & \text { if } \hat{\mu}<0\end{cases}
$$

which limits the model to $\hat{\mu} \geq 0$.

Frequentist techniques are used to test three different scenarios. First, we attempt to exclude a Standard Model (i.e. $\mu=1$ ) Higgs boson at the 95\% confidence level (CL), by testing $\mu<1$. Second, we attempt to discover a Higgs boson $(i . e . \mu \neq 0$ ) with $\geq 5 \sigma$ significance, by testing $\mu>0$. Third, we make a measurement of the signal strength $\mu$ (with a two-sided confidence interval). Each scenario is tested as a raster scan of $m_{H}$.

Under each scenario (detailed below), a test statistic is constructed to quantify the disagreement between the observed data and a null hypothesis. A $p$-value is then computed, which is the probability of observing a test statistic at least as extreme as that observed, under the assumption of the null hypothesis. The $p$-value is computed by integrating a sampling distribution of the test statistic, which is based upon the likelihood function. This is done using an asymptotic formula, which is valid in the large-sample limit, though an ensemble of pseudoexperiments could be used instead [145]. The validity of the asymptotic formula for this analysis has previously been checked against pseudoexperiments.

Finally, the median confidence levels expected under the assumption of an alternative hypothesis are calculated. This is done using a single pseudoexperiment, known as the Asimov dataset, corresponding to the exact expectation (i.e. pseudodata with fractional numbers of events) [145].

\section{Exclusion}

To determine an upper limit on the signal strength, a one-sided test statistic is constructed under the assumption of a signal-plus-background null hypothesis:

$$
\tilde{q}_{\mu}= \begin{cases}-2 \ln \tilde{\lambda}(\mu) & \text { if } \hat{\mu} \leq \mu \\ 0 & \text { if } \hat{\mu}>\mu\end{cases}
$$


where use of $\tilde{\lambda}(\mu)$ ensures that only $\mu \geq 0$ is considered physical. The compatibility of the data with the null hypothesis is quantified by the $p$-value

$$
p_{\mu}=\int_{\tilde{q}_{\mu, \text { obs }}}^{\infty} f\left(\tilde{q}_{\mu} \mid \mu, \hat{\hat{\boldsymbol{\theta}}}(\mu)\right) \mathrm{d} \tilde{q}_{\mu}
$$

where $f\left(\tilde{q}_{\mu} \mid \mu, \hat{\hat{\boldsymbol{\theta}}}(\mu)\right)$ is the sampling distribution of the test statistic under the assumption of an underlying signal strength $\mu$, and $\tilde{q}_{\mu, \text { obs }}$ is the observed test statistic under the same assumption. Thus, a 95\% CL upper limit upon $\mu$ is set at the value which satisfies $p_{\mu}=0.05$

An unfortunate feature of this method is that a downward fluctuation in the observed data can exclude models where little sensitivity is expected. For this reason the modified frequentist $\mathrm{CL}_{s}$ technique is used [147,148], which defines the $p$-value

$$
p_{\mu}^{\prime}=\frac{p_{\mu}}{1-p_{b}}
$$

where incompatibilities with the null hypothesis are down-weighted if the data is also incompatible with the background-only hypothesis, through

$$
p_{b}=\int_{-\infty}^{\tilde{q}_{\mu, \text { obs }}} f\left(\tilde{q}_{\mu} \mid 0, \hat{\hat{\boldsymbol{\theta}}}(0)\right) \mathrm{d} \tilde{q}_{\mu} .
$$

Thus, a 95\% CL upper limit upon $\mu$ is set at the value which satisfies $p_{\mu}^{\prime}=0.05$. The Standard Model Higgs boson is considered excluded if $\mu<1$ at the 95\% CL. The exclusion sensitivity is evaluated by calculating the expected $\mu$ upper limit under the assumption of a background-only alternative hypothesis.

\section{Discovery}

For discovery, a one-sided test statistic is constructed under the assumption of a background-only null hypothesis:

$$
q_{0}= \begin{cases}-2 \ln \lambda(0) & \text { if } \hat{\mu} \geq 0 \\ 0 & \text { if } \hat{\mu}<0\end{cases}
$$


The compatibility of the data with the null hypothesis is quantified by the $p$-value

$$
p_{0}=\int_{q_{0, \mathrm{obs}}}^{\infty} f\left(q_{0} \mid 0, \hat{\hat{\boldsymbol{\theta}}}(0)\right) \mathrm{d} q_{0} .
$$

The Higgs boson is considered discovered if the background-only hypothesis is excluded with $p_{0}<2.87 \times 10^{-7}$, corresponding to a significance of at least five standard deviations (i.e. $\geq 5 \sigma)$ in a Gaussian distribution. The discovery sensitivity is evaluated by calculating the expected $p_{0}$ under the assumption of a signal-plusbackground alternative hypothesis, with $\mu=1$. As seen in Section 8.3, an $m_{H}$ value must also be chosen for this hypothesis.

\section{Measurement}

For the $\mu$ measurement, a two-sided test statistic is constructed under the assumption of a signal-plus-background null hypothesis:

$$
t_{\mu}=-2 \ln \lambda(\mu)
$$

The compatibility of the data with the null hypothesis is quantified by the $p$-value

$$
p_{\mu}=\int_{t_{\mu, \mathrm{obs}}}^{\infty} f\left(t_{\mu} \mid \mu, \hat{\hat{\boldsymbol{\theta}}}(\mu)\right) \mathrm{d} t_{\mu}
$$

The nominal $\mu$ value will be $\hat{\mu}$, and the $68 \%$ CL confidence interval is set by the values which satisfy $p_{\mu}=0.16$. The measurement sensitivity is evaluated by calculating the expected $\Delta \mu$ under the assumption of a signal-plus-background hypothesis, with $\mu=1$. Again, an $m_{H}$ value must be chosen for this hypothesis.

\subsection{Results}

The entire LHC Run I dataset was analysed, corresponding to an integrated luminosity of $4.5 \mathrm{fb}^{-1}$ at $\sqrt{s}=7 \mathrm{TeV}$ and $20.3 \mathrm{fb}^{-1}$ at $\sqrt{s}=8 \mathrm{TeV}$. The major differences of the $7 \mathrm{TeV}$ analysis, with respect to Chapter 4 , are the absence of the $\geq 2$-jet bin and the dilepton triggers, and the use of a dijet fake factor in the $W+$ jet background estimation. Additionally, the number of $m_{\mathrm{T}}$ bins used in the fit is reduced by a factor of two. Some 
minor differences also exist in the object and event selection criteria due to the less harsh pile-up environment at $7 \mathrm{TeV}$ (see Section 3.2.2).

\subsubsection{Exclusion, discovery and measurement of $\mathrm{gg} \rightarrow \mathrm{H} \rightarrow \mathrm{WW}$}

The event selection criteria of the $g g \rightarrow H \rightarrow W W$ analysis are chosen such that the dominant production mode of the selected Higgs boson events is ggF. Nevertheless, other production modes contribute to the signal acceptance. This effect is small in the 0 -jet and 1-jet bins, but VBF ( $V H)$ contributes $15 \%$ (10\%) of signal events to the $\geq 2$-jet bin. Such events are treated as signal in the fit, and their expected yield is scaled by the same $\mu$ as ggF. This has a small effect upon the results, and splitting $\mu$ according to production mode shall be revisited in Section 8.3.2.

The observed and expected (under a background-only hypothesis) 95\% CL upper limit on $\mu$ is shown as a function of the mass under test $m_{H}$ in Figure 8.2a. The mass range where this limit is below unity is excluded at the $95 \% \mathrm{CL}$, when considering an SM Higgs boson $(\mu=1)$. In the absence of a Higgs boson, the expected excluded region is $116 \mathrm{GeV}$ to $200 \mathrm{GeV} .^{1}$ However, the observed excluded region is $132 \mathrm{GeV}$ to $200 \mathrm{GeV}$. The fact that the observed exclusion is weaker than expected indicates an excess of events consistent with a Higgs boson. Since the mass resolution of the $H \rightarrow W W$ analysis is poor, the impact upon the exclusion is broad in $m_{H}$.

To quantify the significance of the excess of events, Figure $8.2 \mathrm{~b}$ shows the observed $p_{0}$ as a function of $m_{H}$. The maximum observed significance is $4.8 \sigma$, which occurs when testing $m_{H}=130 \mathrm{GeV}$, though the poor mass resolution leads to a relatively broad $p_{0}$ curve. This is very strong evidence for the existence of the Higgs boson, though does not pass the $5 \sigma$ criterion for "discovery".

The best-fit signal strength $\hat{\mu}$ is shown as a function of the $m_{H}$ under test in Figure 8.2c. A range of Higgs boson masses are consistent with the assumption that the Higgs boson behaves as predicted by the SM (i.e. $\mu=1)$. The data are also consistent with a lower mass Higgs boson with $\mu>1$ or a higher mass Higgs boson with $\mu<1$.

\footnotetext{
1 This analysis is optimised to search for a low-mass Higgs boson and therefore the region $m_{H}>200 \mathrm{GeV}$ is not considered. A dedicated high-mass search for $H \rightarrow W W$ is described in reference [11].
} 


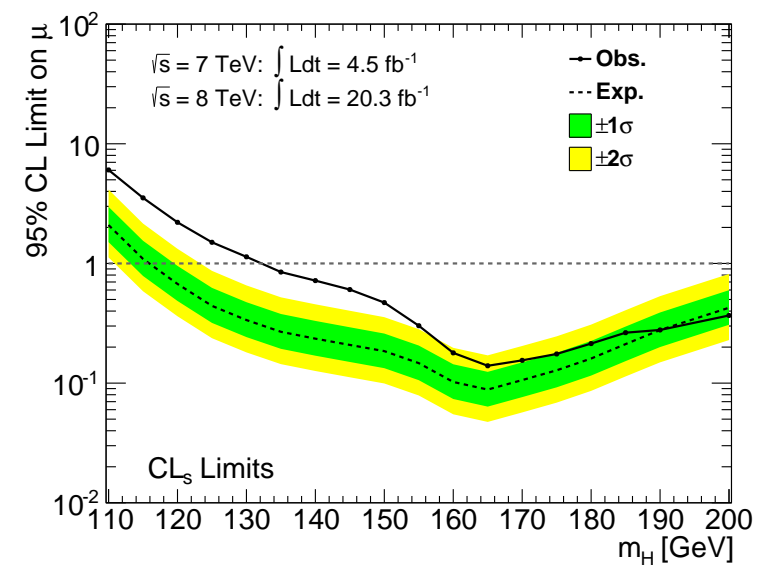

(a) Exclusion

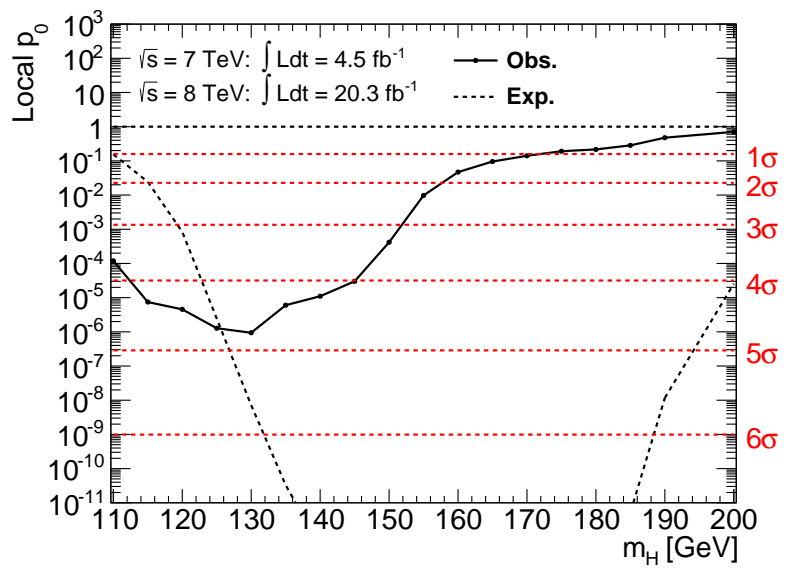

(b) Discovery

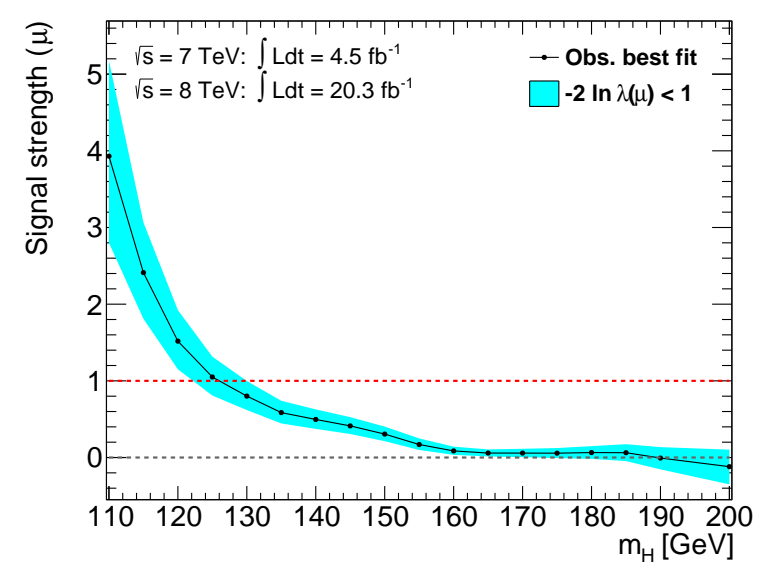

(c) Measurement of $\mu$

Figure 8.2: Results of the ggF analysis. (a) The observed (solid) 95\% CL upper limit on the signal strength $\mu$ as a function of the mass under test $m_{H}$, and the expectation (dashed) under the background-only hypothesis. (b) The observed (solid) $p_{0}$ as a function of $m_{H}$ and the expectation (dashed) under the signal-plus-background hypothesis with $\mu=1$ and a hypothesised mass equal to that under test. (c) The best-fit signal strength $\hat{\mu}$ (solid) as a function of $m_{H}$, with the $68 \%$ CL interval shown (blue band). 


\subsubsection{Combination with VBF analysis}

The ggF analysis described in this thesis is combined with a multivariate VBF analysis, which selects events featuring two high- $p_{\mathrm{T}}$ jets separated by a large rapidity gap devoid of hadronic activity. This analysis features a higher signal-to-background ratio than the $\mathrm{ggF}$ analysis, but has a smaller expected number of events. It was briefly introduced in Section 4.3.7, because the $\geq 2$-jet bin of the ggF analysis vetoes events selected by the VBF analysis in order to maintain orthogonality. The VBF analysis will be fully described in the upcoming paper [9].

The 95\% CL upper limit on $\mu$ is shown as a function of $m_{H}$ in Figure 8.3a. The expected (in the absence of a Higgs boson) excluded region is $114 \mathrm{GeV}$ to $200 \mathrm{GeV}$, exhibiting only a small improvement upon the limit from the ggF analysis. The observed excluded region is $132 \mathrm{GeV}$ to $200 \mathrm{GeV}$.

The observed $p_{0}$ is shown in Figure $8.3 \mathrm{~b}$ as a function of the mass under test $m_{H}$. The maximum observed significance is $6.1 \sigma$, occurring when testing $m_{H}=130 \mathrm{GeV}$. This constitutes a discovery of the Higgs boson, made by searching for the ggF and VBF production modes and the $W W$ decay channel.

The best-fit signal strength $\hat{\mu}$ is shown as a function of the $m_{H}$ under test in Figure 8.3c. By including $m_{H}$ as a parameter of interest in the fit, it is possible to test which $\left(\mu, m_{H}\right)$ pair are most favoured by the data. This is presented in Figure 8.3d, together with likelihood contours. The best fit values are $\left(\hat{\mu}, \hat{m}_{H}\right)=(0.90,128 \mathrm{GeV})$.

These results exhibit more than $5 \sigma$ significance for $H \rightarrow W W \rightarrow \ell \nu \ell \nu$, and consequently for the existence of the Higgs boson itself. LHC searches for $H \rightarrow \gamma \gamma$ and $H \rightarrow Z Z$ have found consistent evidence, which is summarised in Section 9.1. These two channels yield much better mass sensitivity, and observe $m_{H} \approx 125 \mathrm{GeV}$. For this reason, the expected $p_{0}$ and $\hat{\mu}$ under the assumption of a SM Higgs boson with $m_{H}=125 \mathrm{GeV}$ are also included in Figures 8.3b and 8.3c respectively. It can be seen that the observed $p_{0}$ is smaller than expected (i.e. the significance of the incompatibility with the null hypothesis is greater), though the shape remains consistent. The best-fit signal strength at $m_{H}=125 \mathrm{GeV}$ is observed to be

$$
\hat{\mu}=1.11 \pm 0.22 \text {. }
$$




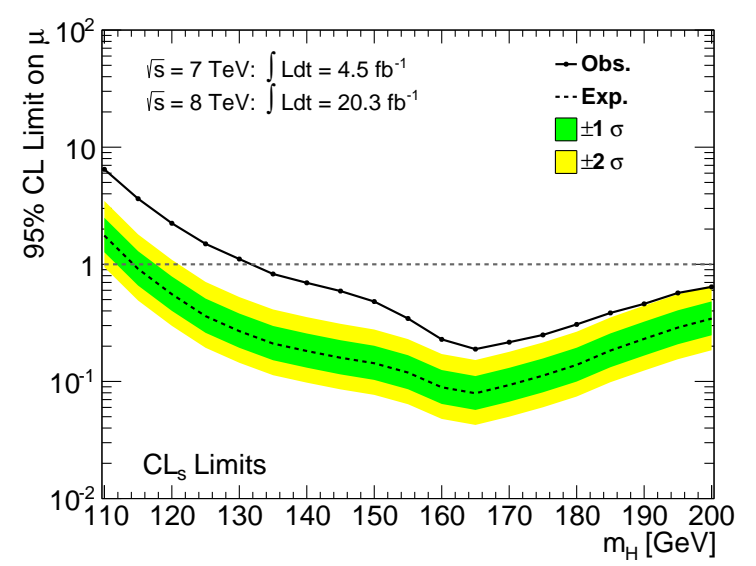

(a) Exclusion

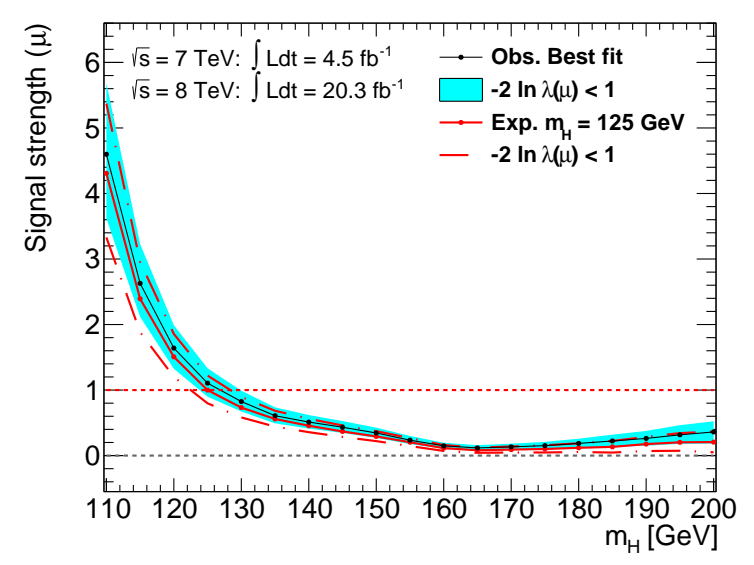

(c) Measurement of $\mu$

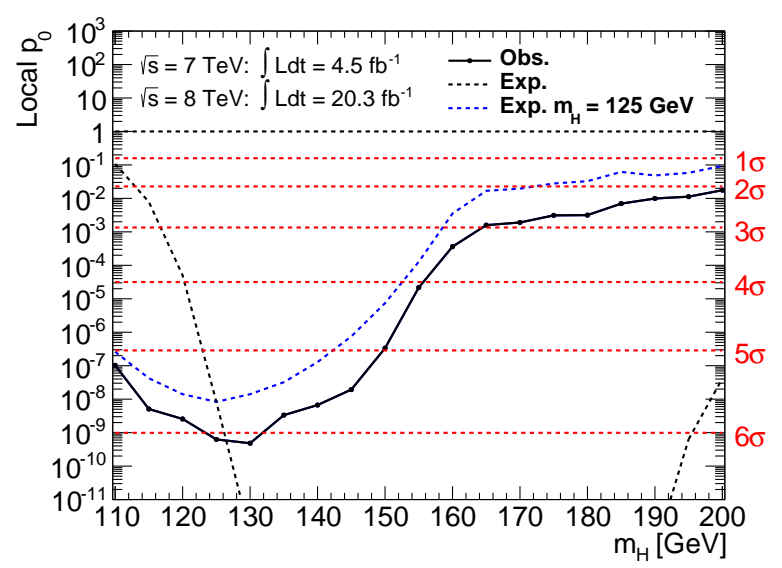

(b) Discovery

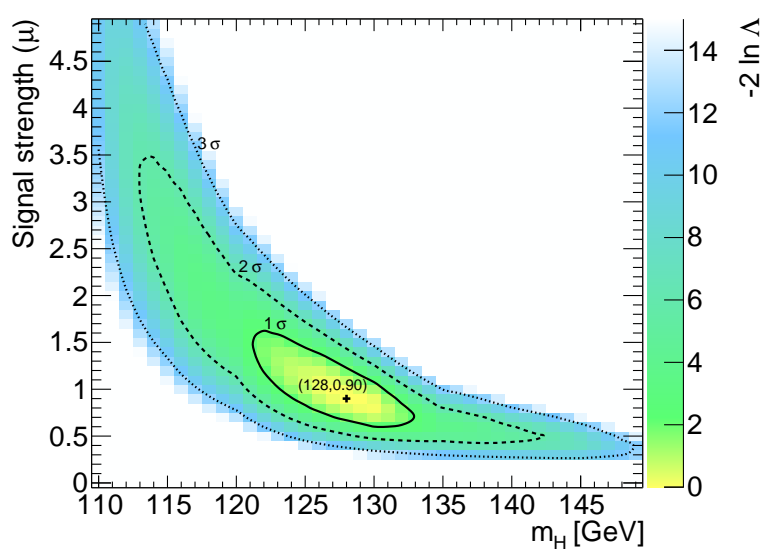

(d) Measurement of $\left(\mu, m_{H}\right)$

Figure 8.3: Results of the combined ggF+VBF analysis. (a) The observed (solid) $95 \% \mathrm{CL}$ upper limit on the signal strength $\mu$ as a function of the mass under test $m_{H}$, and the expectation (dashed) under the background-only hypothesis. (b) The observed (solid) $p_{0}$ as a function of $m_{H}$ and the expectation (black dashed) under the signal-plus-background hypothesis with $\mu=1$ and a hypothesised mass equal to that under test. The expectation (blue dashed) for a hypothesised mass $m_{H}=125 \mathrm{GeV}$ is also shown. (c) The best-fit signal strength $\hat{\mu}$ (solid) as a function of $m_{H}$, with the $68 \% \mathrm{CL}$ interval shown (blue band). The expectation (red) for a hypothesised mass $m_{H}=125 \mathrm{GeV}$ is also shown. (d) The best-fit signal strength $\hat{\mu}$ and mass $\hat{m}_{H}$ (marker), with the likelihood contours also shown. 
The excess of events is further characterised in Figure 8.4. In comparison, the combined $H \rightarrow W W$ analysis performed by the CMS experiment measures $\hat{\mu}=0.72 \pm 0.19$ at $m_{H}=125.6 \mathrm{GeV}[149]$.

It is possible to assign the ggF and VBF production modes independent signal strength parameters, with $\mu_{\mathrm{ggF}}$ constrained by the ggF analysis and $\mu_{\mathrm{VBF}}$ constrained by the VBF analysis. This is interesting because they are dominated by different couplings, and the loop in the ggF process can be sensitive to undiscovered massive particles. When doing this, the $V H$ production mode is assigned the same signal strength as VBF because they are both dominated by bosonic couplings. The resulting limits upon $\left(\mu_{\mathrm{ggF}}, \mu_{\mathrm{VBF}}\right)$ are shown in Figure 8.5. It can be seen that both ggF and VBF are in excellent agreement with the SM, though VBF is observed to be produced more often than expected. The best-fit values are $\left(\hat{\mu}_{\mathrm{ggF}}, \hat{\mu}_{\mathrm{VBF}}\right)=(0.99,1.32)$.

Table 8.2 shows a breakdown of the uncertainties in $\hat{\mu}$. It can be seen that $\hat{\mu}_{\mathrm{VBF}}$ is dominated by statistical uncertainties, whereas $\hat{\mu}_{\mathrm{ggF}}$ is almost equally limited by statistical and systematic uncertainties. It should be noted that theoretical uncertainties (particularly in signal modelling) are the largest source of systematic uncertainty, highlighting the importance of the ggF and $W W$ studies in Chapters 5 and 6 respectively. 


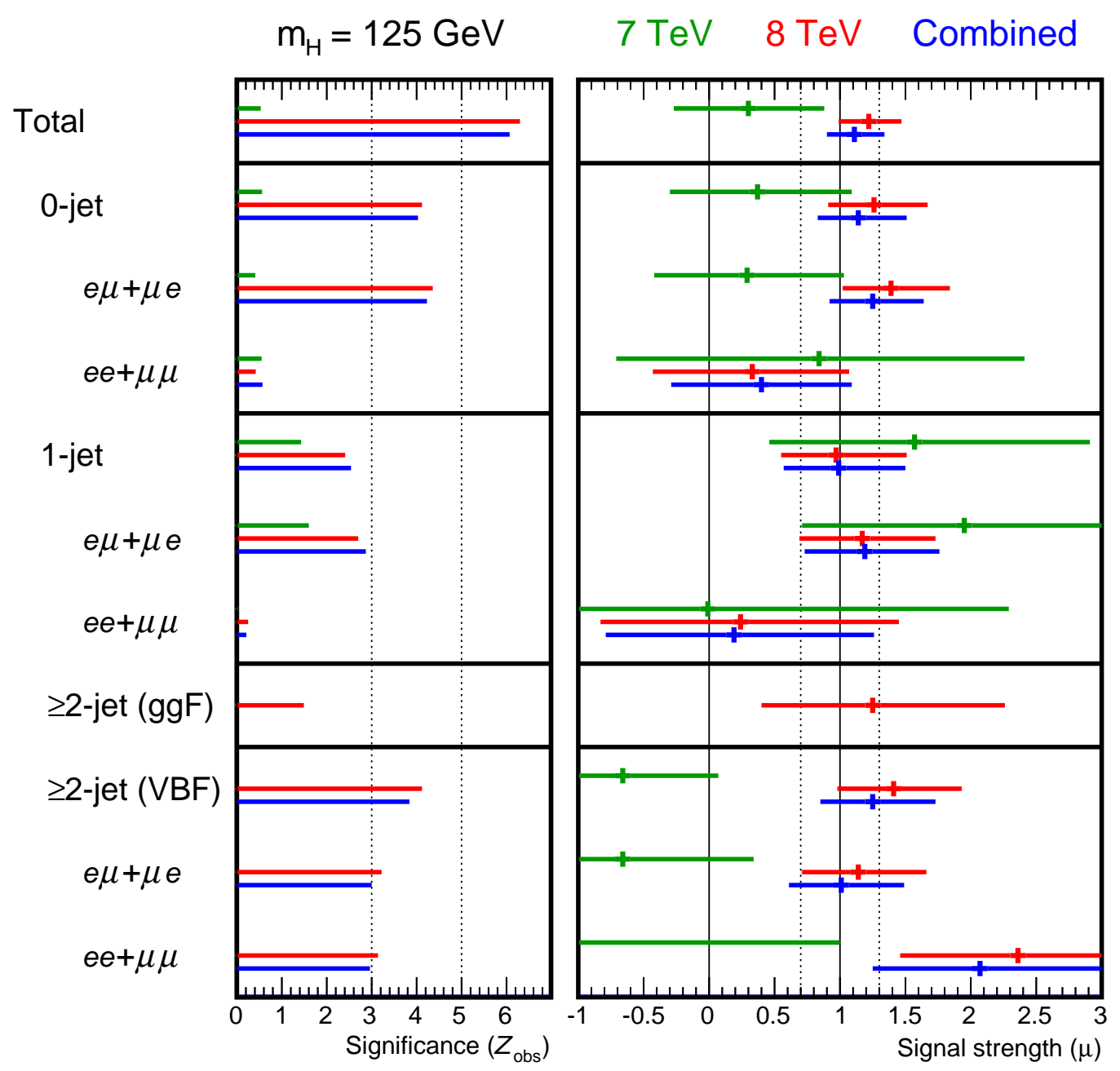

Figure 8.4: The observed significance in units of Gaussian standard deviations, $Z$, and the measured signal strength, $\hat{\mu}$, when testing $m_{H}=125 \mathrm{GeV}$. The contribution of each signal region is shown. The fit results using the $\sqrt{s}=7 \mathrm{TeV}$ dataset (green), the $\sqrt{s}=8 \mathrm{TeV}$ dataset (red), and their combination (blue) are also separated. 


\begin{tabular}{|c|c|c|c|}
\hline & \multicolumn{3}{|c|}{ Relative uncertainty $\Delta \mu / \mu(\%)$} \\
\hline & $\operatorname{ggF}$ & VBF & Combined \\
\hline Statistical & 20 & 33 & 16 \\
\hline Signal regions & 14 & 29 & 12 \\
\hline Control regions & 12 & 15 & 9 \\
\hline Signal contamination & 3 & 7 & 0 \\
\hline Finite MC size & 6 & 4 & 4 \\
\hline Systematic & 18 & 21 & 13 \\
\hline Experimental & 10 & 13 & 8 \\
\hline Leptons and triggers & 5 & 2 & 4 \\
\hline Jets and $b$-tagging & 3 & 10 & 2 \\
\hline$p_{\mathrm{T}}^{\mathrm{inv}}$ modelling & 2 & 4 & 2 \\
\hline Fake factor & 8 & 2 & 5 \\
\hline Other & 4 & 5 & 3 \\
\hline Theoretical & 15 & 16 & 11 \\
\hline Signal modelling & 11 & 14 & 9 \\
\hline Background modelling & 9 & 8 & 7 \\
\hline Luminosity & 3 & 4 & 3 \\
\hline Total & 26 & 39 & 20 \\
\hline
\end{tabular}

Table 8.2: A breakdown of the relative uncertainty upon the fitted signal strengths $\hat{\mu}_{\mathrm{ggF}}$ and $\hat{\mu}_{\mathrm{VBF}}$, and in the combined signal strength $\hat{\mu}$. "Signal contamination" refers to how the finite number of events in the signal region of the VBF analysis introduces a statistical uncertainty in the expected number of VBF events in the signal region of the ggF analysis, and vice versa. Uncertainties are symmetrised by averaging the high and low variations. 


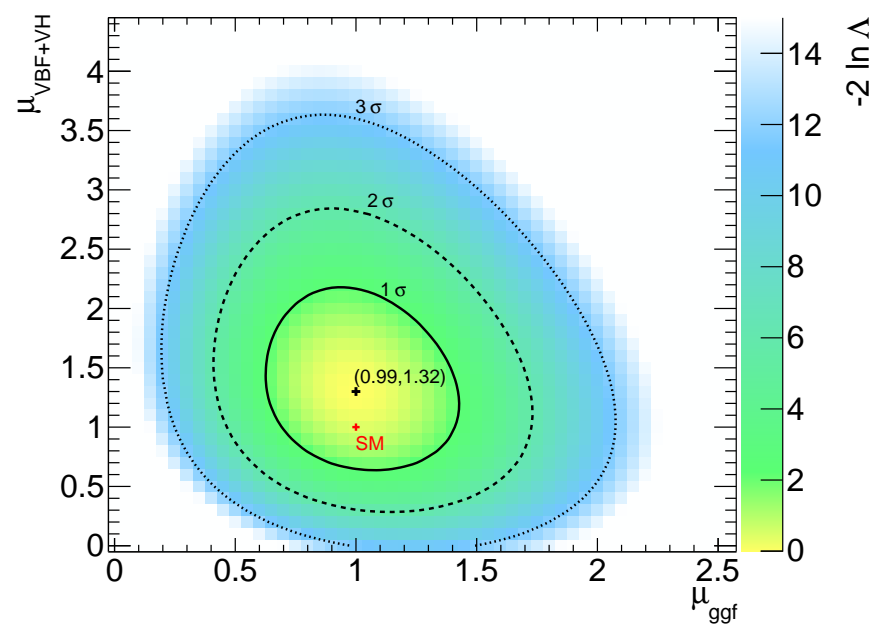

Figure 8.5: Likelihood contours for $\mu_{\mathrm{ggF}}$ and $\mu_{\mathrm{VBF}+\mathrm{VH}}$, when testing $m_{H}=125 \mathrm{GeV}$. The markers show the best-fit values observed (black) and expected under an $m_{H}=125 \mathrm{GeV}$ hypothesis (red). 


\subsubsection{Cross section measurements}

Following the method described in Section 5.2.2, fiducial ggF cross sections are extracted for fiducial regions defined for the 0 -jet and 1-jet bins of the $e \mu+\mu e$ channel. These are only extracted at $\sqrt{s}=8 \mathrm{TeV}$. This is done using

$$
\begin{aligned}
\sigma_{\mathrm{ggF}}^{\mathrm{fid}} & =\frac{N_{\mathrm{obs}}-N_{\mathrm{bkg}}}{C_{\mathrm{ggF}} \cdot L} \\
& =\hat{\mu}_{\mathrm{ggF}} \cdot \sigma_{\mathrm{ggF}}^{\mathrm{SM}} \cdot \mathrm{BR}^{\mathrm{SM}} \cdot A_{\mathrm{ggF}}
\end{aligned}
$$

where $L$ is the luminosity, $C_{\mathrm{ggF}}$ is the ratio of the expected number of ggF events passing the detector-level selection to those passing the fiducial selection, and $A_{\mathrm{ggF}}$ is the ratio of the expected number of ggF events passing the fiducial selection to the total expected number of ggF events. Here, $\hat{\mu}$ is determined by a dedicated fit in which the nuisance parameters associated with theoretical uncertainties in the total ggF cross section, the $\mathrm{BR}$ and the ggF acceptance are fixed, such that their effect upon $\Delta \mu$ is removed. The $\mathrm{VBF}$ and $V H$ processes are set to their SM expectations (i.e. $\mu_{\mathrm{VBF}}=1$ ). The measured fiducial cross sections for $m_{H}=125 \mathrm{GeV}$ are displayed in Table 8.3. The measured values are higher than predicted, which is consistent with Figure 8.4.

The product of the total cross section and the $H \rightarrow W W$ branching ratio is extracted for ggF (at $\sqrt{s}=7 \mathrm{TeV}$ and $8 \mathrm{TeV}$ ) and $\mathrm{VBF}($ at $\sqrt{s}=8 \mathrm{TeV}$ ) using

$$
\begin{aligned}
\sigma_{i} \cdot \mathrm{BR} & =\frac{N_{\mathrm{obs}}-N_{\mathrm{bkg}}}{C_{i} \cdot A_{i} \cdot L} \\
& =\hat{\mu}_{i} \cdot \sigma_{i}^{\mathrm{SM}} \cdot \mathrm{BR}^{\mathrm{SM}}
\end{aligned}
$$

where $i=g g F, V B F$. The $\hat{\mu}_{i}$ are determined by a dedicated fit in which the nuisance parameters associated with theoretical uncertainties in the total cross sections and branching ratio are fixed, such that their effect is removed. When $\hat{\mu}_{\mathrm{ggF}}$ is measured $\mu_{\mathrm{VBF}}$ is profiled, and vice versa. The measured cross sections for $m_{H}=125 \mathrm{GeV}$ are displayed in Table 8.4 and show good agreement with the theoretical predictions. 


\begin{tabular}{crr}
\hline Fiducial & \multicolumn{2}{c}{$\sigma^{\text {fid }}(\mathrm{fb})$} \\
region & Measured & Predicted \\
\hline 0-jet $e \mu+\mu e$ & $28.1 \pm 5.5 \pm 4.1$ & $19.9 \pm 3.3$ \\
1-jet $e \mu+\mu e$ & $8.4 \pm 3.1 \pm 1.9$ & $7.3 \pm 1.8$ \\
\hline
\end{tabular}

Table 8.3: Measured fiducial ggF cross sections at $\sqrt{s}=8 \mathrm{TeV}$, assuming $m_{H}=125 \mathrm{GeV}$. Theoretical predictions are shown for comparison. The uncertainties in measured quantities are statistical and systematic, respectively.

\begin{tabular}{ccccc}
\hline \multirow{2}{*}{ Process } & $\sqrt{s}$ & \multicolumn{3}{c}{$\sigma \cdot \mathrm{BR}(\mathrm{pb})$} \\
& & Measured & Predicted \\
\hline $\operatorname{ggF}$ & $7 \mathrm{TeV}$ & $1.7 \pm 1.8 \pm 1.2$ & 3.3 & \pm 0.4 \\
$\mathrm{ggF}$ & $8 \mathrm{TeV}$ & $4.7 \pm 0.8 \pm 0.8$ & 4.1 & \pm 0.5 \\
$\mathrm{VBF}$ & $8 \mathrm{TeV}$ & $0.53 \pm 0.16 \pm 0.10$ & $0.339 \pm 0.017$ \\
\hline
\end{tabular}

Table 8.4: Measured ggF and VBF total cross sections multiplied by the $H \rightarrow W W$ branching ratio, assuming $m_{H}=125 \mathrm{GeV}$. Theoretical predictions are shown for comparison. The uncertainties in measured quantities are statistical and systematic, respectively. 


\section{Chapter 9}

\section{Status of Higgs physics}

The analysis presented in this thesis has found significant experimental evidence for the process $g g \rightarrow H \rightarrow W W \rightarrow \ell \nu \ell \nu$. However, it was on 4th July 2012 that the ATLAS and CMS collaborations independently announced the discovery of a new particle consistent with the Higgs boson of the Standard Model (SM), using a combination of decay channels to reject the null hypothesis with more than $5 \sigma$ significance $[4,150]$. Further $p p$ collisions were recorded until the end of December 2012, and then the entire Run I dataset was used to study the new particle in detail.

Section 9.1 summarises the most important measurements of this new particle, which confirm that it has the qualitative properties of the Higgs boson of the SM. The theoretical implications of the discovery are considered in Section 9.2. Finally, in Section 9.3, the outlook of Higgs boson measurements is assessed in the short and long term future.

\subsection{Properties of the discovered Higgs boson}

For a particle to be considered "discovered" within the particle physics community, the null hypothesis must be rejected with $\geq 5 \sigma$ significance, corresponding to $<0.0001 \%$ chance that the observation is a statistical fluctuation. In the summer of 2012, this level of significance was reached independently by the ATLAS and CMS collaborations, primarily driven by searches for $H \rightarrow \gamma \gamma$ and $H \rightarrow Z Z$ and supported by the $H \rightarrow W W$ search [4,150]. Both experiments observed a resonance at $m \approx 125 \mathrm{GeV}$.

This observation was quickly corroborated by $V H \rightarrow V b \bar{b}$ searches performed by the CDF and D $\varnothing$ experiments at the Tevatron, which yielded consistent results with $3.1 \sigma$ combined significance [151]. 
Using the entire Run I dataset, the significance of the particle discovery was extended by the ATLAS experiment to $5.2 \sigma$ in $H \rightarrow \gamma \gamma$ [152], 8.1 $\sigma$ in $H \rightarrow Z Z$ [153], and $6.1 \sigma$ in $H \rightarrow W W$ (see Chapter 8). Focus inevitably shifted to measuring its properties (mass, couplings, spin, parity) in order to test the hypothesis that it is a Higgs boson. Observations confirm this hypothesis, though more precise measurements are required to ascertain whether or not it is the Higgs boson predicted by the SM.

\subsubsection{Mass measurement}

The mass of the Higgs boson, $m_{H}$, is unpredicted by the SM, and is very important to measure precisely. Once $m_{H}$ is known, all the Higgs boson couplings, production cross sections and branching ratios can be calculated (see Section 1.3). The value of $m_{H}$ is also important when assessing the theoretical implications of the discovery (see Section 9.2).

The $H \rightarrow \gamma \gamma$ and $H \rightarrow Z Z$ decay channels offer the best sensitivity to $m_{H}$, since they exhibit very clean experimental signatures and allow full reconstruction of the Higgs boson four-momentum. A combination of these two channels results in a measured mass of $125.36 \pm 0.37$ (stat) \pm 0.18 (syst) GeV by the ATLAS collaboration [154] and $125.7 \pm 0.3$ (stat) \pm 0.3 (syst) GeV by the CMS collaboration [155]. These are in excellent agreement, although some tension exists between the two ATLAS channels. The Higgs boson is as heavy as an atom of caesium-133.

A Higgs boson with $m_{H} \approx 125 \mathrm{GeV}$ is fortuitous, since many different production modes and decay channels should be experimentally accessible at the LHC (see Figures 1.4 and 1.5). In particular, couplings to both bosons and fermions can be measured, directly testing the two types of mass generation the Higgs field is responsible for in the SM.

\subsubsection{Coupling measurements and limits}

Coupling strengths of the Higgs boson to other SM particles are probed by searching for different experimental signatures, which correspond to specific combinations of production mode and decay channel. For example, the $W H$ production mode with the $H \rightarrow W W$ decay mode is sensitive only to the $H W W$ coupling. On the other hand, the ggF production mode and the $H \rightarrow \gamma \gamma$ decay channel feature loops, and interpreting results in terms of couplings is less straightforward. Undiscovered massive particles may contribute to such loops, distorting the effective couplings to gluons and photons. 


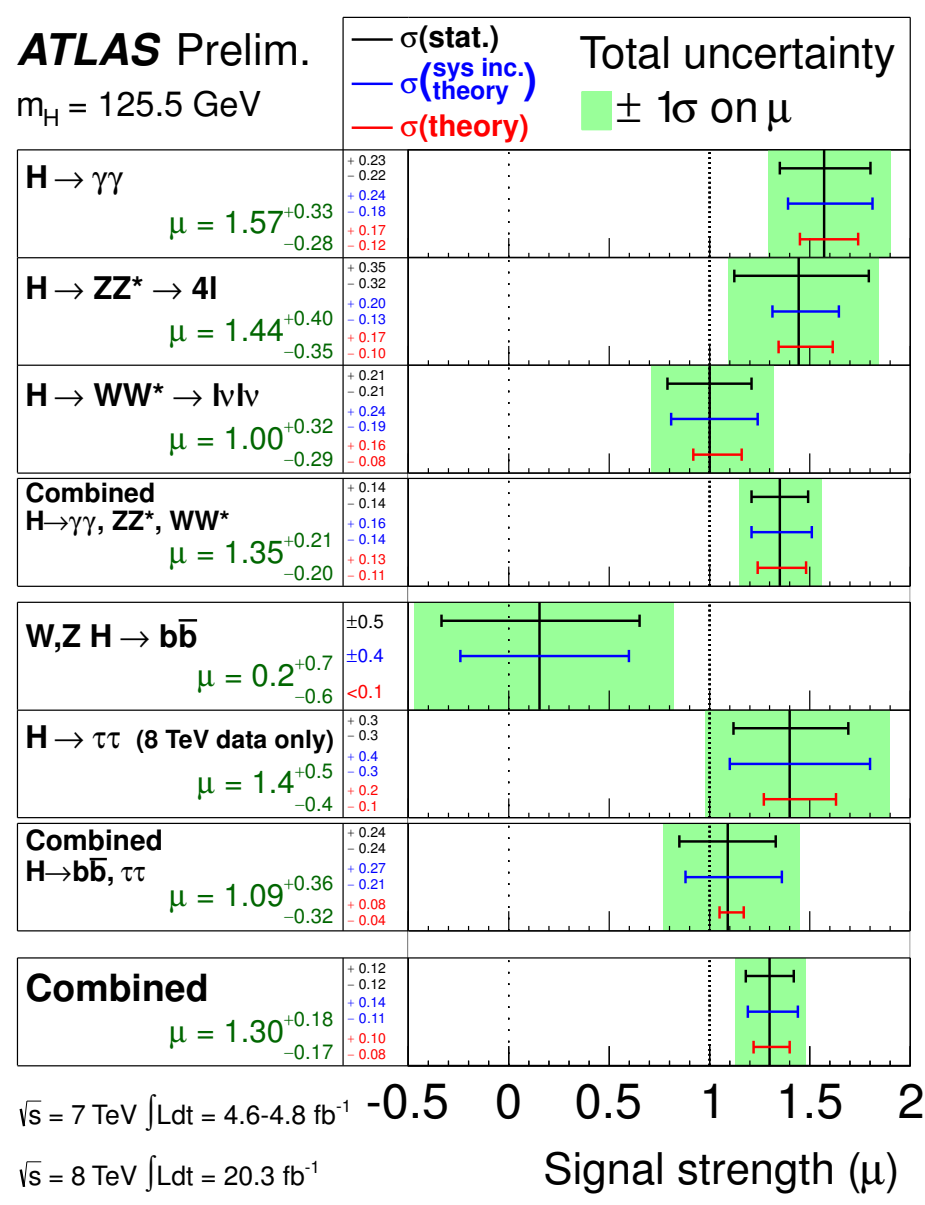

Figure 9.1: Measured signal strength $\left(m_{H}=125.5 \mathrm{GeV}\right)$ for each final state [160]. Combinations of bosonic, fermionic and all decay channels are also shown. The $H \rightarrow W W$ result is from a previous analysis of the Run I dataset, before the final sensitivity optimisation described in this thesis.

Measurements of $H \rightarrow \gamma \gamma, H \rightarrow Z Z, H \rightarrow W W$ and $H \rightarrow \tau \tau$ were made with the Run I dataset, with signal regions optimised for ggF and VBF production. A measurement of $V H \rightarrow V b \bar{b}$ was also made. Assuming SM couplings, the measured cross sections can be expressed as a signal strength $\mu=\sigma_{\text {meas }} / \sigma_{\mathrm{SM}}$. The signal strengths measured with the ATLAS experiment are consistent with the SM predictions, as shown in Figure 9.1. Similar measurements were made with the CMS detector, also yielding signal strengths compatible with the SM [149, 156-159].

It is also informative to split the signal strength according to the production mode. Figure 9.2 shows the likelihood contours for a fit with two signal strength parameters: one for production modes dominated by a fermionic coupling ( $g g \mathrm{~F}$ and $t t H$ ), and the other for modes dominated by a bosonic coupling (VBF and $V H$ ). Again, this assumes 


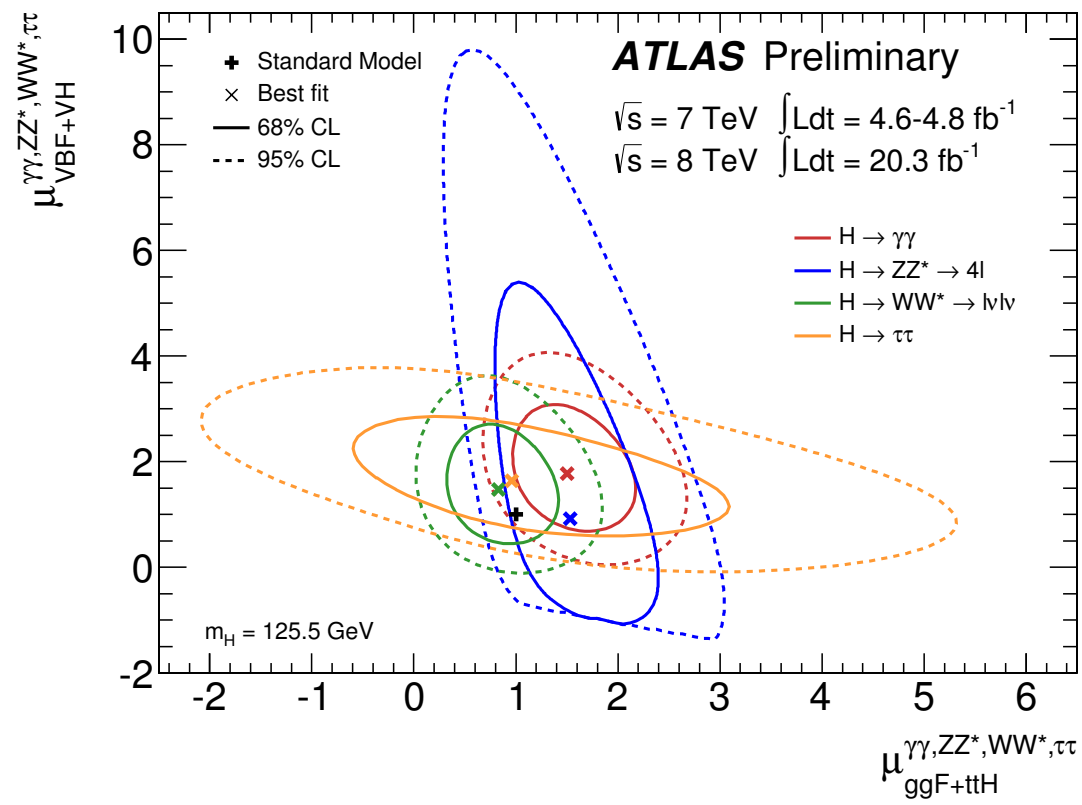

Figure 9.2: Likelihood contours of measured signal strength $\left(m_{H}=125.5 \mathrm{GeV}\right)$ for each final state [160]. Signal strengths are split according to the dominant coupling in the production mode: fermionic ( $\mathrm{ggF}$ and $t t H)$ or bosonic (VBF and $V H$ ). The $H \rightarrow W W$ result is from a previous analysis of the Run I dataset.

the couplings themselves are those predicted by the SM, but it is useful to see which analyses are sensitive to which types of couplings. It is clear that $H \rightarrow W W$ is a very sensitive analysis for coupling measurements.

These measurements were used to test specific coupling scenarios in reference [160], and no significant deviations from the SM were observed. The two most generic scenarios are highlighted here.

The first scenario assumes a SM particle content. The probed couplings are free parameters in the fit, and are expressed as coupling scale factors: $\kappa_{Z}, \kappa_{W}, \kappa_{t}, \kappa_{b}, \kappa_{\tau}$. The $H \gamma \gamma$ and $H g g$ effective couplings and the total width are then calculated within the framework of the SM, as functions of the $\kappa_{i}$. Compatibility with the SM is $p=0.13$ (see Figure 9.3a), with differences driven by the $H \rightarrow b \bar{b}$ result.

The second scenario allows for undiscovered particles, which may contribute through loops or to the total width. Thus, two effective coupling scale factors are introduced $\left(\kappa_{\gamma}, \kappa_{g}\right)$, and the absence of a total width constraint means that only coupling ratios can be probed. The free parameters of the fit are $\lambda_{\gamma Z}, \lambda_{W Z}, \lambda_{b Z}, \lambda_{\tau Z}, \lambda_{g Z}, \lambda_{t g}, \kappa_{g Z}$ where $\lambda_{i j}=\kappa_{i} / \kappa_{j}, \kappa_{i j}=\kappa_{i} \cdot \kappa_{j} / \kappa_{H}$ and $\kappa_{H}$ is the scale factor of the total width. Compatibility 


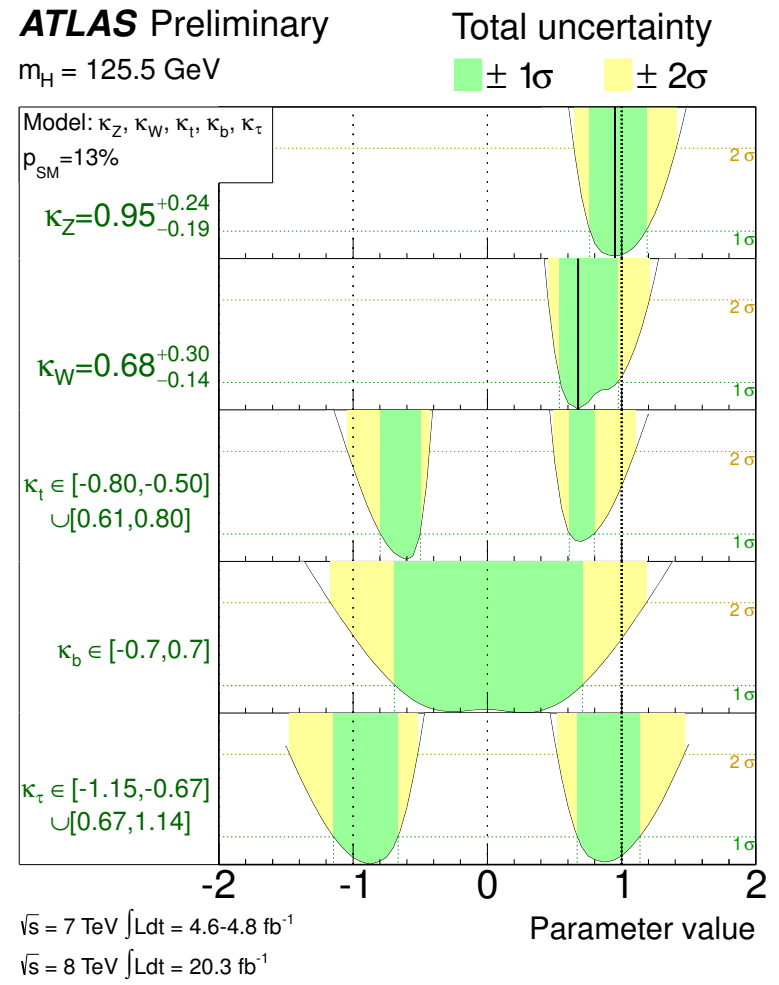

(a) SM particle content

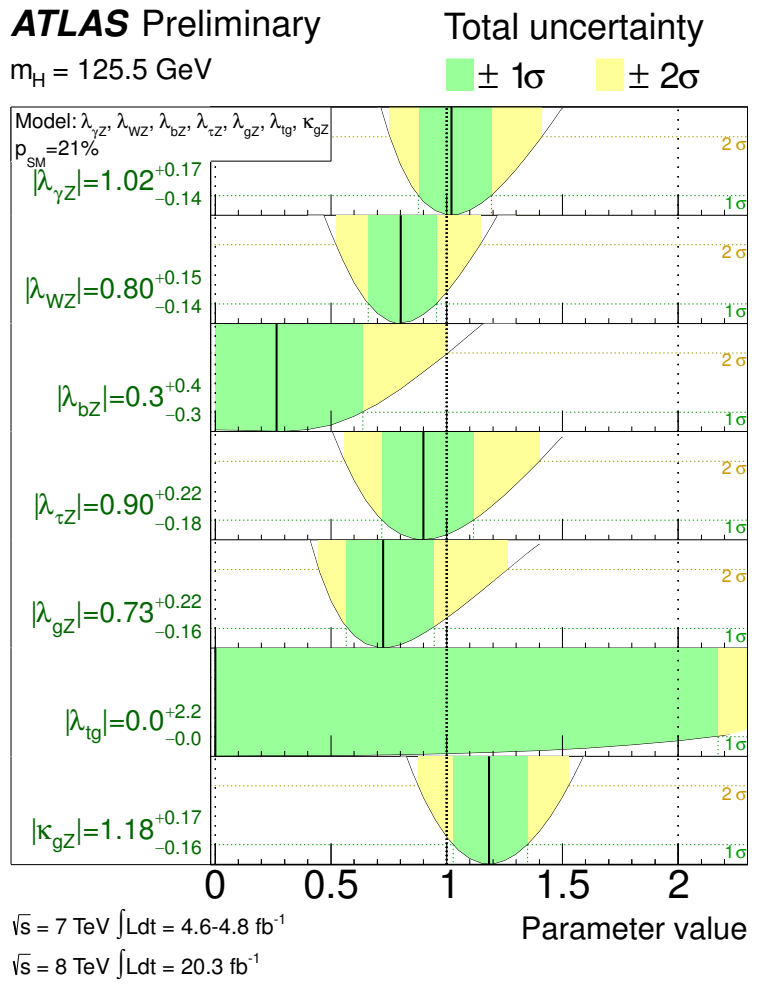

(b) SM + undiscovered particle content

Figure 9.3: Measured likelihood curves of coupling scale factors $\kappa_{i}$ and ratios $\lambda_{i j}=\kappa_{i} / \kappa_{j}$, for two generic models described in the text [160].

with the SM is $p=0.21$ (see Figure $9.3 \mathrm{~b}$ ). The large uncertainty on $\lambda_{t g}$ could be improved by a measurement of the $t t H$ production mode.

In addition to the five measured channels described above, upper bounds on rare decay channels have also been set by ATLAS. The observed limits at $m_{H}=125 \mathrm{GeV}$ are $\sigma<11 \sigma_{\mathrm{SM}}$ for $H \rightarrow Z \gamma$ [161] and $\sigma<7 \sigma_{\mathrm{SM}}$ for $H \rightarrow \mu \mu$ [162], at the $95 \% \mathrm{CL}$. Also, a search for $Z H \rightarrow \ell \ell p_{\mathrm{T}}^{\text {inv }}$ constrained the branching ratio to invisible particles to $<75 \%$ at the $95 \% \mathrm{CL}$, assuming $\sigma=\sigma_{\mathrm{SM}}$ and $m_{H}=125 \mathrm{GeV}$ [163]. Such a limit is important because undiscovered massive particles that interact weakly (e.g. dark matter) could enhance the invisible width $\Gamma_{\text {inv }}$. Slightly tighter limits have been set by the CMS collaboration [164-166]. 


\subsubsection{Spin and parity measurement}

The SM Higgs boson is a spin-0 and CP-even particle, i.e. $J^{P}=0^{+}$. To establish that the observed particle is the Higgs boson, it is important to experimentally confirm these properties. According to the Landau-Yang theorem, the spin-1 hypothesis is excluded by the observation of $H \rightarrow \gamma \gamma[167,168]$. Since there may be coincident resonances, this hypothesis is tested by the other channels.

The $J^{P}$ was probed by considering the decay topologies of events in the $H \rightarrow Z Z$, $H \rightarrow W W$ and $H \rightarrow \gamma \gamma$ measurements with ATLAS. However, some event selection criteria were altered with respect to the coupling measurements, in order to improve sensitivity. For example, the $H \rightarrow W W$ selection exploits the spin-0 hypothesis through the $m_{\ell \ell}<55 \mathrm{GeV}$ and $\Delta \phi(\ell, \ell)<1.8$ criteria (see Section 4.3.4).

The $0^{-}, 1^{+}, 1^{-}, 2^{+}$hypotheses are excluded at greater than $97.8 \% \mathrm{CL}$, whilst the data are compatible with the $0^{+}$hypothesis [169]. This provides strong evidence that the discovered particle is spin-0, and is the only observed fundamental scalar particle. It could be a mixture of CP-even and CP-odd states, though a preference for CP-even is observed.

\subsection{Theoretical implications}

The discovery of the Higgs boson appears to "complete" the SM in the most minimal way, with no significant deviations from predictions observed. That its $H W W$ and $H Z Z$ couplings agree with expectations confirms that the Higgs mechanism underlies electroweak symmetry breaking. That its fermionic couplings are proportional to mass supports that fermion masses are generated by Yukawa interactions.

In Sections 9.2.1 and 9.2.2, the discovered Higgs boson shall be interpreted assuming that the SM is valid up to the Planck energy scale (at which point gravity becomes strong and dominates phenomenology). Then, Section 9.2.3 considers why the observed particle might indicate that new physics should be observed at lower scales. 


\subsubsection{Global electroweak fit}

In Section 1.4.2, a global fit of electroweak data was used to motivate a low mass Higgs boson. The discovery of the Higgs boson and the measurement of its mass overconstrains the electroweak theory, allowing a test of its validity.

The updated fit exhibits a $p$-value of 0.176 , corresponding to a deviation from the Standard Model of significance $1.35 \sigma$ [170]. Thus, the experimental data included in the fit are consistent with electroweak theory. The pulls of individual fit parameters are shown in Figure 9.4; the dominant tension is unrelated to $m_{H}$. The measurement of $m_{H}$ does cause some tension with the measured $m_{W}$ and $m_{t}$, which are sensitive to $m_{H}$ through loop corrections.

\subsubsection{Vacuum stability}

In Section 1.4.3, theoretical arguments were used to constrain $m_{H}$ under the assumption that the Standard Model is valid up to the reduced Planck scale $\bar{\Lambda}_{\mathrm{P}} \sim 10^{18} \mathrm{GeV}$. Requiring the Higgs quartic coupling $\lambda$ remain perturbative implied $m_{H}<175 \mathrm{GeV}$, while requiring the electroweak vacuum to remain a stable minimum implied $m_{H}>129 \mathrm{GeV}$ [48].

The measurement of $m_{H} \approx 125 \mathrm{GeV}$ excludes the stability of the SM vacuum at 98.6\% CL [171]. However, a potential barrier separates the vacuum in which the Universe currently resides and the true SM vacuum. The probability of quantum tunnelling through this barrier is sufficiently small that the lifetime of the Universe far exceeds its age. Thus, measurements suggest that we exist in a metastable vacuum (see Figure 9.5).

The fact that experimental measurements place the Universe very close to the critical boundary for vacuum stability means that $\lambda$ and $\beta_{\lambda}$ are both very close to zero at the instability scale. The potential significance of this observation is an active area of theoretical research. One interesting idea also uses the scalar nature of the Higgs boson to lend credence to slow-roll models of cosmic inflation with the Higgs boson acting as the inflaton. However, minimal configurations of such models may fail to predict the power spectrum of anisotropies observed in the cosmic microwave background $[172,173]$. 


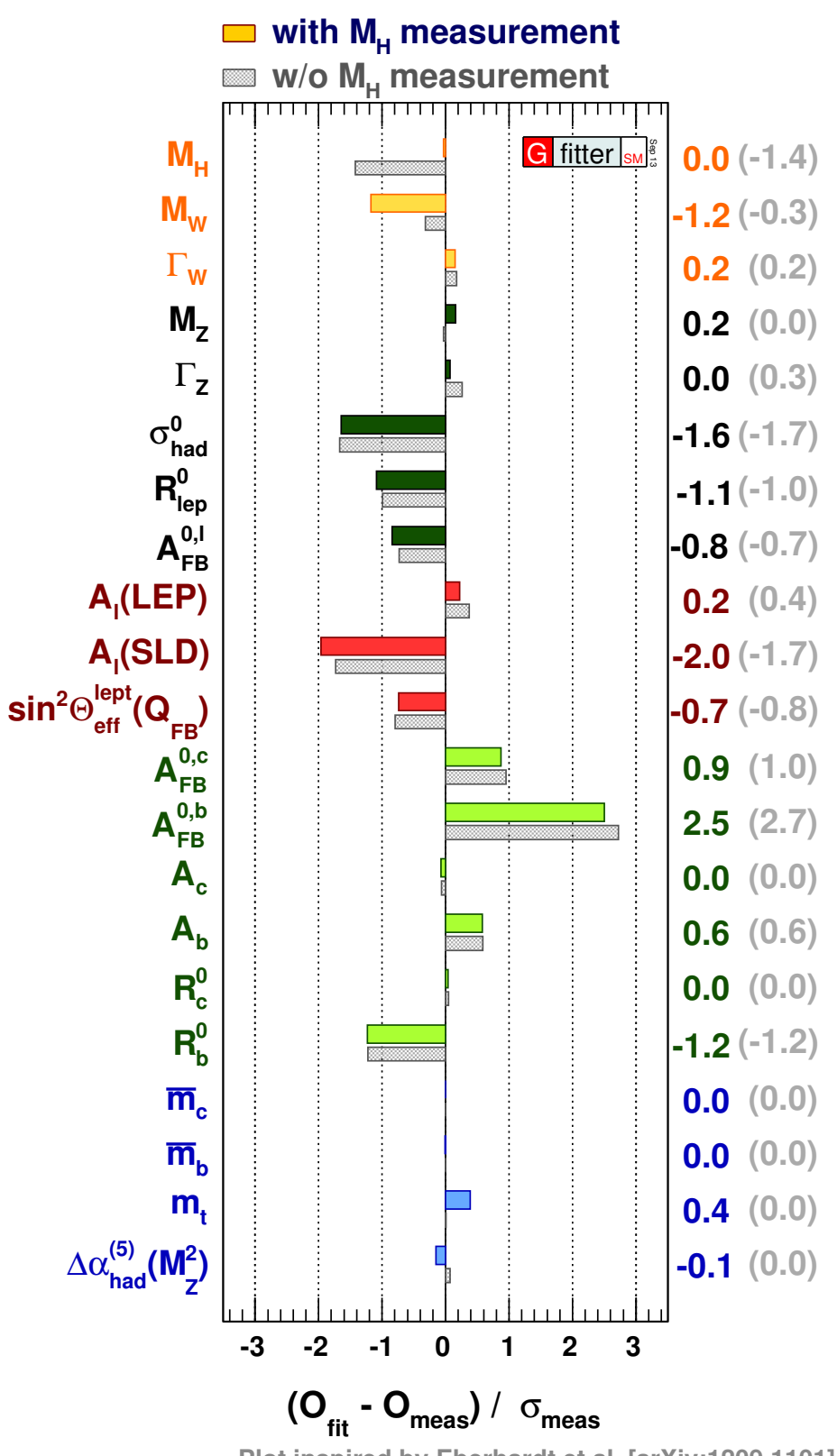

Figure 9.4: Pull values of the electroweak fit parameters, with (colour) and without (grey) the $m_{H}$ measurement included [170]. The pull value is the deviation of the fitted value from the experimental measurement, in units of the experimental uncertainty. 

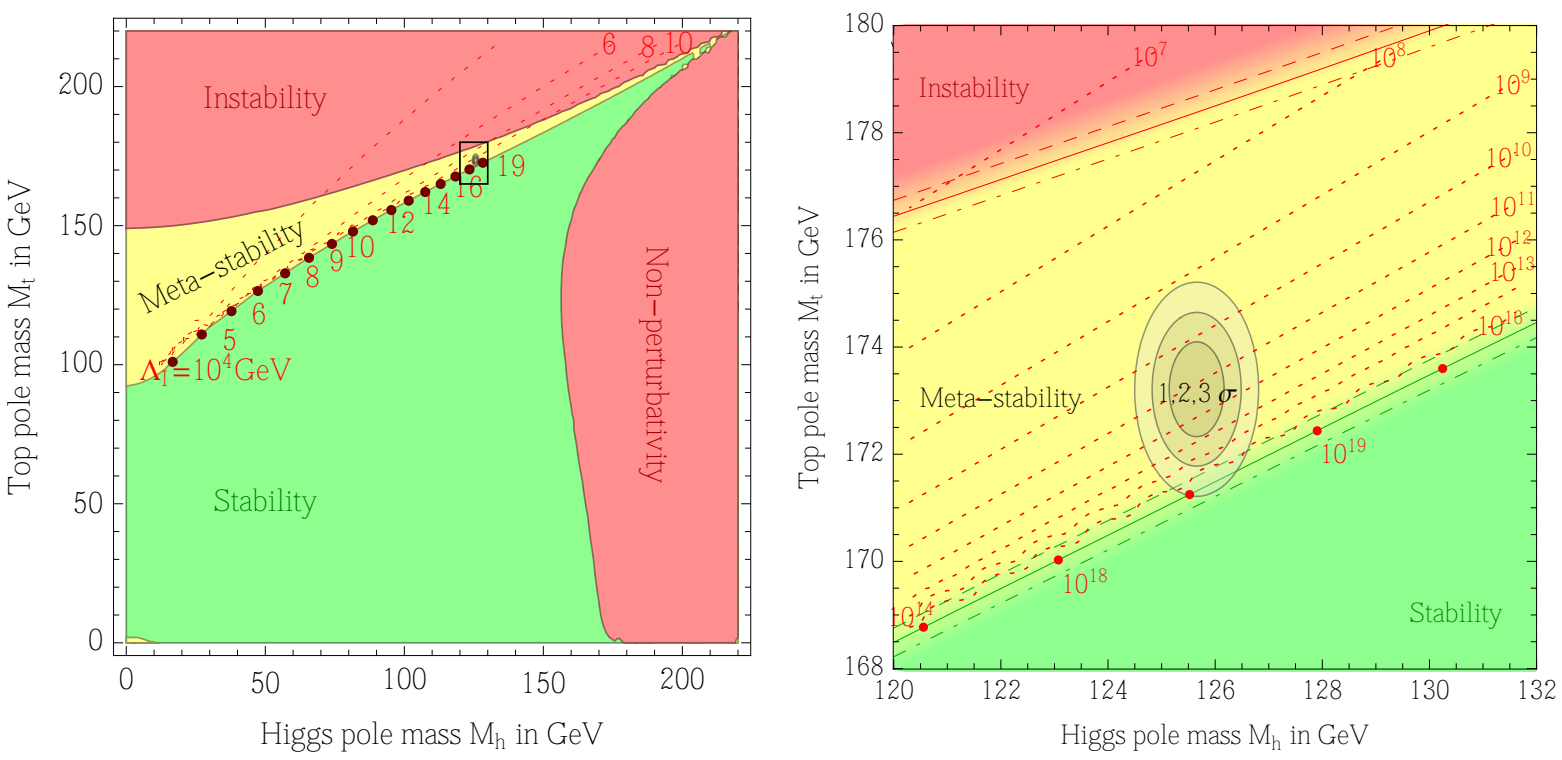

Figure 9.5: Phase diagram of the Standard Model, expressed in terms of $m_{H}$ and $m_{t}$ [171]. The phases correspond to stable, metastable and unstable vacuum states and a non-perturbative Higgs quartic coupling $\lambda$, assuming that the scale at which new physics is introduced is $\Lambda_{\mathrm{NP}}=\Lambda_{\mathrm{P}} \sim 10^{19} \mathrm{GeV}$. Dotted lines indicate the scale at which the instability phase transition occurs. A zoomed version (right) elucidates the experimentally measured situation.

\subsubsection{The hierarchy problem}

The Higgs boson acquires a mass through oscillations about the non-zero vacuum expectation value of the Higgs field. However, this tree-level mass is subject to loop corrections containing massive particles; the top quark loop dominates, though the $W$, $Z$ and Higgs bosons also contribute significantly. Similar corrections to $m_{W}$ and $m_{t}$ were used to constrain $m_{H}$ in Section 1.4.2.

Such loop diagrams should in principle be calculated to infinitely high scale, introducing ultraviolet (UV) divergences. They can be calculated within the SM for scales up to $\Lambda_{\mathrm{P}} \sim 10^{19} \mathrm{GeV}$, but a theory of everything (ToE) would be needed above $\Lambda_{\mathrm{P}}$. These corrections to $m_{H}$ are quadratically divergent, and so the measured $m_{H}$ can be 
schematically written as

$$
\begin{aligned}
m_{H, \text { physical }}^{2} & =m_{H, \text { bare }}^{2}+\int_{0}^{\Lambda_{\mathrm{P}}} \mathrm{SM} \text { loops }+\int_{\Lambda_{\mathrm{P}}}^{\infty} \text { ToE loops } \\
& =m_{H, \text { bare }}^{2}+\mathcal{O}\left(\Lambda_{\mathrm{P}}^{2}\right)+\int_{\Lambda_{\mathrm{P}}}^{\infty} \mathrm{ToE} \text { loops } .
\end{aligned}
$$

The bare mass $m_{H \text {,bare }}$ is not predicted by the SM, but when combined with the ToE loops it must largely cancel the $\mathcal{O}\left(\Lambda_{\mathrm{P}}^{2}\right)$ term, leaving behind $m_{H \text {,physical }}^{2}=(125 \mathrm{GeV})^{2}$. This requires the ToE to produce a fine tuning of 1 part in $\left(\Lambda_{\mathrm{P}} / m_{H}\right)^{2} \sim 10^{34}$, which is highly unnatural.

One may ask why the hierarchy problem is specific to the Higgs boson, and the same fine tuning is not required for other particles. This is because corrections to the masses of other particles are only logarithmically divergent (rather than quadratically divergent); their masses are protected by gauge or chiral symmetries.

If new physics exists at a scale $\Lambda_{\mathrm{NP}} \ll \Lambda_{\mathrm{P}}$, say $\mathcal{O}(1 \mathrm{TeV})$, the degree of fine tuning can be reduced dramatically. This is a primary motivation for many new physics models. Supersymmetry introduces a new symmetry between fermions and scalars, which protects $m_{H}$ [174]. Technicolour regards the Higgs boson as a composite state [175]. Models with extra dimensions assume that gravity is actually strong, but $\Lambda_{\mathrm{P}}$ appears large because the gravitational flux is diluted in the extra dimensions [176].

Thus, the existence of the Higgs boson motivates new physics at a scale $\mathcal{O}(1 \mathrm{TeV})$. However, no experimental evidence in support of the candidate models has been found at the LHC, and indeed many configurations have been excluded.

\subsection{Outlook}

In the short term future, CERN expects to operate Run II of the LHC in 2015 - 2017, and Run III in 2019 - 2021. Run II is designed to deliver $\sim 100 \mathrm{fb}^{-1}$ at $\sqrt{s}=13-14 \mathrm{TeV}$, whilst Run III is designed to deliver $\sim 300 \mathrm{fb}^{-1}$ at $\sqrt{s}=14 \mathrm{TeV}$. In the long term future, 
CERN plans to further upgrade the LHC instantaneous luminosity (HL-LHC), to start running in 2023 and deliver $\sim 3000 \mathrm{fb}^{-1}$ at $\sqrt{s}=14 \mathrm{TeV} .^{1}$

Compared to $\sqrt{s}=8 \mathrm{TeV}$, the Higgs boson production cross sections at $\sqrt{s}=14 \mathrm{TeV}$ are $\sim 2.5$ times larger for ggF, $\mathrm{VBF}$ and $V H$, and $\sim 4.7$ times larger for $t t H$. Many of the background processes increase by a smaller factor, which should aid the sensitivity of analyses. However, the harsher pile-up environment will degrade detector performance. These considerations, together with the large expected luminosities, should yield more precise signal strength measurements and enable observations of rare decays (e.g. $H \rightarrow Z \gamma$ and $H \rightarrow \mu \mu$ ) and additional production channels (e.g. $t t H)$. Prospects for the $300 \mathrm{fb}^{-1}$ and $3000 \mathrm{fb}^{-1}$ datasets were investigated by ATLAS [177], and the expected precision of signal strengths and coupling scale factors are shown in Figure 9.6.

The total width is predicted to be $\Gamma_{H}=4.07 \pm 0.16 \mathrm{MeV}$ at $m_{H}=125 \mathrm{GeV}$ [10]. Although this is not experimentally resolvable, it should be possible to indirectly constrain $\Gamma_{H}$ at future LHC runs. The Higgs boson propagator $1 /\left(\left(\hat{s}-m_{H}^{2}\right)^{2}+m_{H}^{2} \Gamma_{H}^{2}\right)$ indicates that the on-shell production cross section is sensitive to $\Gamma_{H}$, though it is difficult to disentangle this information from coupling scale factors. However, by comparing the off-shell region to the on-shell region, it is possible to constrain $\Gamma_{H}$ with $H \rightarrow Z Z$ and $H \rightarrow W W$ events [178-180]. Also, interference between signal and continuum background can produce a $\Gamma_{H}$-dependent shift in $m_{H}$, which could be measured at the HL-LHC with $H \rightarrow \gamma \gamma$ events $[181,182]$.

At the HL-LHC it should be possible to measure diHiggs production, which would yield a first direct measurement of the Higgs self-coupling $\lambda$ [183]. This could prove important in understanding the metastability of the vacuum (see Section 9.2.2). A measurement of triHiggs production would allow the final Higgs term in the Lagrangian to be measured, though this has an extremely small cross section.

Finally, a solution to the hierarchy problem (see Section 9.2.3) shall be sought in future LHC runs. This is a very large topic in itself, though Higgs measurements will play their role in this. Additional (sometimes charged) Higgs bosons often feature in such models, and so searches for these shall continue. Also, it shall be important to further constrain the invisible Higgs width, which can act as a probe of dark matter candidates.

\footnotetext{
1 Another post-LHC scenario is to upgrade the LHC energy to $\sqrt{s}=33 \mathrm{TeV}$ (HE-LHC). Highly speculative longer term ideas include building a $100 \mathrm{~km}$ tunnel beneath Geneva to house a $\sqrt{s}=1 \mathrm{TeV}$ $e^{+} e^{-}$collider (TLEP), and then later a $\sqrt{s}=100 \mathrm{TeV} p p$ collider. The international community is also considering proposals for $e^{+} e^{-}$linear colliders, ILC and CLIC, which would have $\sqrt{s} \sim 1 \mathrm{TeV}$.
} 
ATLAS Simulation Preliminary

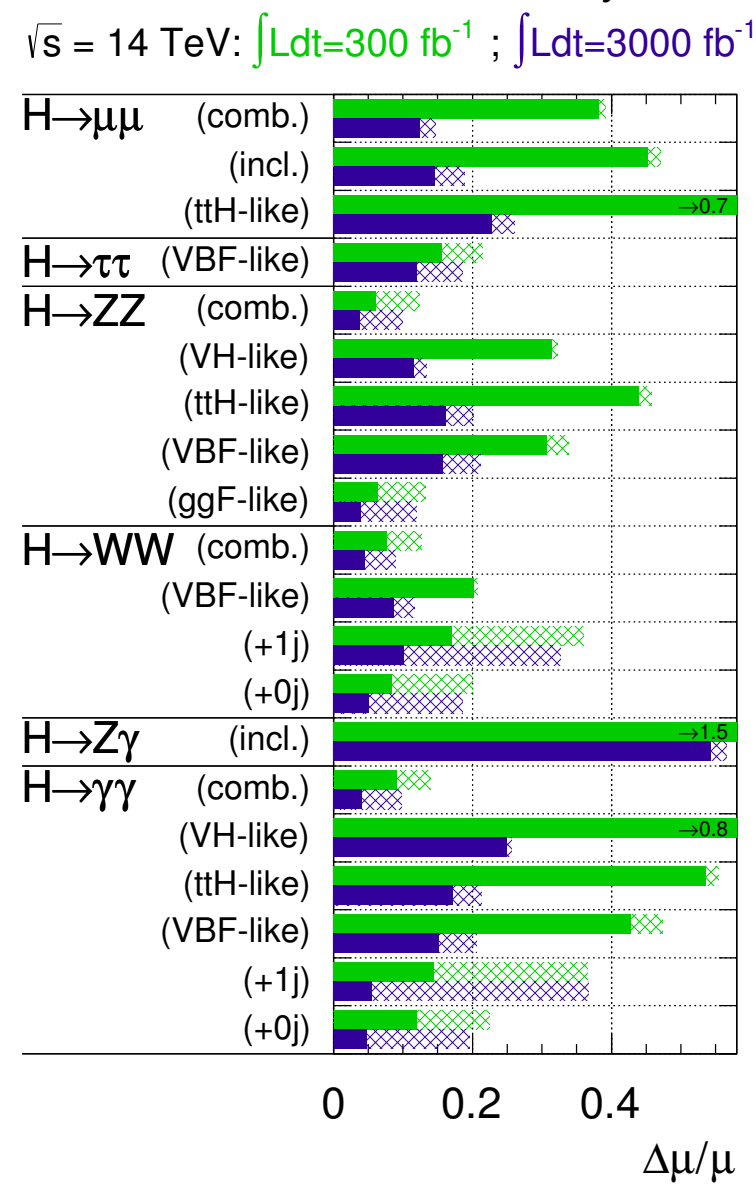

ATLAS Simulation Preliminary

$\sqrt{\mathrm{s}}=14 \mathrm{TeV}: \int \mathrm{Ldt}=300 \mathrm{fb}^{-1} ; \int \mathrm{Ldt}=3000 \mathrm{fb}^{-1}$

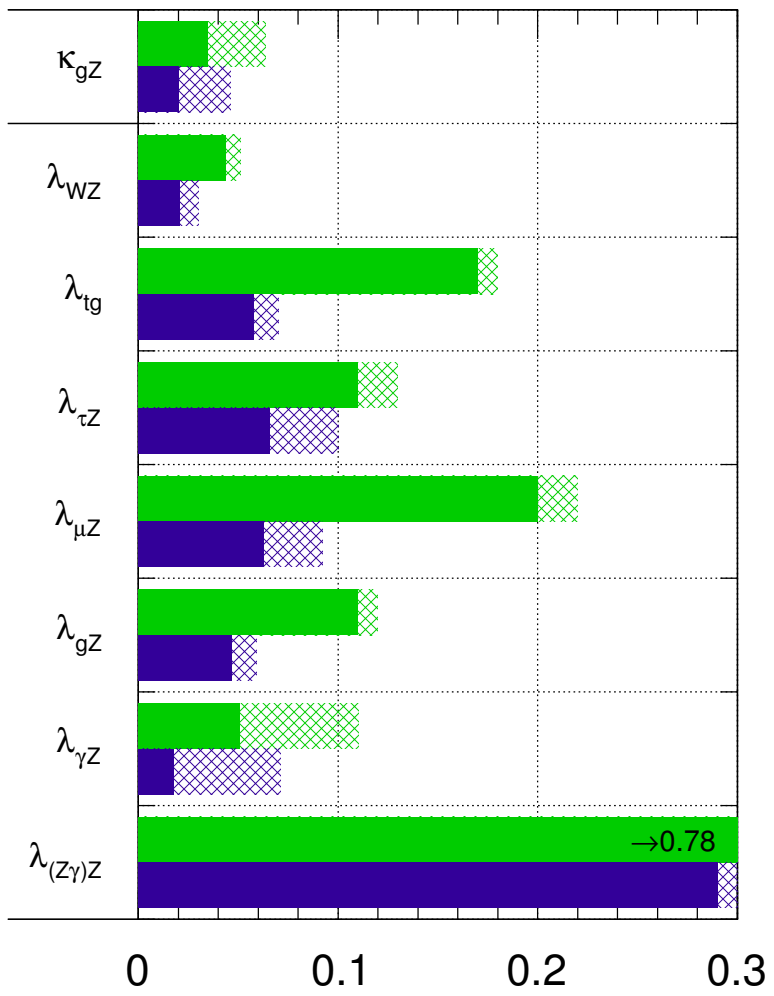

$\Delta \lambda_{\mathrm{XY}}=\Delta\left(\frac{\kappa_{\mathrm{X}}}{\kappa_{\mathrm{Y}}}\right)$

Figure 9.6: Expected precision of Higgs boson measurements at Run III of the LHC (green) and the HL-LHC (blue), assuming $m_{H}=125 \mathrm{GeV}$ [177]. The left shows signal strength precision for a variety of experimental signatures. The right shows the precision of coupling scale factor ratios, following the second generic parametrisation described in Section 9.1.2. The hatched areas indicate the decrease in precision due to theoretical uncertainties.

The discovery of the Higgs boson has initiated a new era in high energy physics. It confirms the Higgs mechanism of electroweak symmetry breaking and the mass generation of fermions via Yukawa interactions, whilst appearing to leave the Standard Model intact and self-consistent. However, it does present interesting opportunities to probe models of new physics. 


\section{Chapter 10}

\section{Conclusions}

This thesis has described the experimental search for the $g g \rightarrow H \rightarrow W W \rightarrow \ell \nu \ell \nu$ process of Higgs boson production and decay. It uses the LHC Run I dataset of $p p$ collisions recorded by the ATLAS detector, which corresponds to an integrated luminosity of $4.5 \mathrm{fb}^{-1}$ at $\sqrt{s}=7 \mathrm{TeV}$ and $20.3 \mathrm{fb}^{-1}$ at $\sqrt{s}=8 \mathrm{TeV}$. An excess of events is observed with a significance of 4.8 standard deviations $(4.8 \sigma)$, which is consistent with Higgs boson production. The significance is extended to $6.1 \sigma$ when the vector boson fusion production process is included. According to the convention adopted by the particle physics community, this constitutes a first observation, or discovery, of this process. The observed resonance is found to be consistent with the Higgs boson of the Standard Model with $m_{H}=125 \mathrm{GeV}$, as are results from other LHC search channels described in Section 9.1 .

The best-fit signal strength at $m_{H}=125 \mathrm{GeV}$ is found to be $\hat{\mu}=1.11 \pm 0.22$, in excellent agreement with the Standard Model expectation. With a precision of $20 \%$, this $H \rightarrow W W$ analysis is the most sensitive $\mu$ measurement of the LHC Run I Higgs boson analyses $[149,152,153,156,157]$. It will take some years to improve upon this precision at Run II of the LHC. With the expected Run II dataset of $100 \mathrm{fb}^{-1}$ at $\sqrt{s}=13-14 \mathrm{TeV}$, the statistical uncertainty should be reduced - dramatically so for $\mu_{\mathrm{VBF}}$. The larger dataset should also afford more sophisticated background estimation techniques to be employed, e.g. using same-sign events to model the normalisation and shape of the non- $W W$ diboson background, in order to reduce the associated theoretical uncertainties. Finally, advances in theoretical calculations and $\mathrm{MC}$ event generators shall improve the estimation of processes with large theoretical uncertainties (e.g. ggF and $W W$ ). 


\section{Bibliography}

[1] ATLAS Collaboration, Search for the Standard Model Higgs boson in the $H \rightarrow W W \rightarrow \ell \nu \ell \nu$ decay mode with $4.7 \mathrm{fb}^{-1}$ of ATLAS data at $\sqrt{\mathrm{s}}=7 \mathrm{TeV}$, ATLAS-CONF-2012-012 (2012).

[2] ATLAS Collaboration, Search for the Standard Model Higgs boson in the $H \rightarrow W W^{(*)} \rightarrow \ell \nu \ell \nu$ decay mode using Multivariate Techniques with $4.7 \mathrm{fb}^{-1}$ of ATLAS data at $\sqrt{s}=7$ TeV, ATLAS-CONF-2012-060 (2012).

[3] ATLAS Collaboration, Search for the Standard Model Higgs boson in the $H \rightarrow W W^{(*)} \rightarrow \ell \nu \ell \nu$ decay mode with $4.7 \mathrm{fb}^{-1}$ of ATLAS data at $\sqrt{s}=7 \mathrm{TeV}$, Phys. Lett. B 716, 62 (2012), arXiv:1206.0756 [hep-ex].

[4] ATLAS Collaboration, Observation of a new particle in the search for the Standard Model Higgs boson with the ATLAS detector at the LHC, Phys. Lett. B 716, 1 (2012), arXiv: 1207.7214 [hep-ex].

[5] ATLAS Collaboration, Observation of an Excess of Events in the Search for the Standard Model Higgs Boson in the $H \rightarrow W W^{(*)} \rightarrow \ell \nu \ell \nu$ Channel with the ATLAS Detector, ATLAS-CONF-2012-098 (2012).

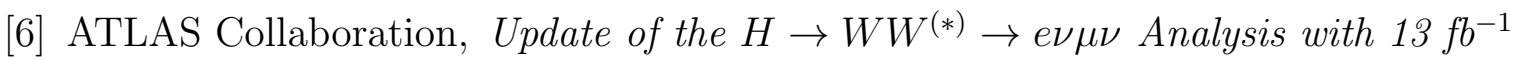
of $\sqrt{s}=8$ TeV Data Collected with the ATLAS Detector, ATLAS-CONF-2012-158 (2012).

[7] ATLAS Collaboration, Measurements of the properties of the Higgs-like boson in the $W W^{(*)} \rightarrow \ell \nu \ell \nu$ decay channel with the ATLAS detector using $25 \mathrm{fb}^{-1}$ of proton-proton collision data, ATLAS-CONF-2013-030 (2013).

[8] ATLAS Collaboration, Measurements of Higgs boson production and couplings in diboson final states with the ATLAS detector at the LHC, Phys. Lett. B 726, 88 (2013), arXiv:1307.1427 [hep-ex]. 
[9] ATLAS Collaboration, Observation and measurement of Higgs boson decays to $W W^{*}$ with ATLAS at the LHC, (2014), in preparation for Phys. Rev. D.

[10] LHC Higgs Cross Section Working Group, Handbook of LHC Higgs Cross Sections: 3. Higgs Properties, CERN-2013-004 (2013), arXiv:1307.1347 [hep-ph].

[11] ATLAS Collaboration, Search for a high-mass Higgs boson in the $H \rightarrow W W \rightarrow \ell \nu \ell \nu$ decay channel with the ATLAS detector using $21 \mathrm{fb}^{-1}$ of proton-proton collision data, ATLAS-CONF-2013-067 (2013).

[12] ATLAS Collaboration, Measurement of the $W W$ cross section in $\sqrt{s}=7$ TeV $p p$ collisions with ATLAS, Phys. Rev. Lett. 107, 041802 (2011), arXiv:1104.5225 [hep-ex].

[13] ATLAS Collaboration, Measurement of the $W^{+} W^{-}$production cross section in proton-proton collisions at $\sqrt{s}=7$ TeV with the ATLAS detector, ATLAS-CONF-2011-110 (2011).

[14] ATLAS Collaboration, Measurement of the $W W$ cross section in $\sqrt{s}=7$ TeV pp collisions with the ATLAS detector and limits on anomalous gauge couplings, Phys. Lett. B 712, 289 (2012), arXiv:1203.6232 [hep-ex].

[15] ATLAS Collaboration, Measurement of $W^{+} W^{-}$production in pp collisions at $\sqrt{s}=7$ TeV with the ATLAS detector and limits on anomalous $W W Z$ and $W W \gamma$ couplings, Phys. Rev. D 87, 112001 (2013), arXiv:1210.2979 [hep-ex].

[16] F. Vasey et al., The Versatile Link common project: feasibility report, JINST 7, C01075 (2012).

[17] D. Hall, B. T. Huffman, and A. Weidberg, The radiation induced attenuation of optical fibres below $-20^{\circ} \mathrm{C}$ exposed to lifetime $H L$-LHC doses at a dose rate of $700 \mathrm{~Gy}(\mathrm{Si}) / \mathrm{hr}$, JINST 7, C01047 (2012).

[18] D. C. Hall, P. Hamilton, B. T. Huffman, P. K. Teng, and A. R. Weidberg, The radiation tolerance of MTP and LC optical fibre connectors to $500 \mathrm{kGy}(\mathrm{Si})$ of gamma radiation, JINST 7, P04014 (2012).

[19] I. J. R. Aitchison and A. J. G. Hey, Gauge Theories in Particle Physics, 3rd ed. (Taylor \& Francis, Abingdon, UK, 2003).

[20] M. E. Peskin and D. V. Schroeder, An Introduction to Quantum Field Theory (Westview Press, 1995). 
[21] S. L. Glashow, Partial-symmetries of weak interactions, Nucl. Phys. 22, 579 (1961).

[22] S. Weinberg, A Model of Leptons, Phys. Rev. Lett. 19, 1264 (1967).

[23] A. Salam, Weak and Electromagnetic Interactions, in Elementary particle physics: relativistic groups and analyticity, edited by N. Svartholm, p. 367, Almqvist \& Wiksell, 1968, Proceedings of the eighth Nobel symposium.

[24] G. 't Hooft and M. J. G. Veltman, Regularization and renormalization of gauge fields, Nucl. Phys. B 44, 189 (1972).

[25] E. Noether, Invariante Variationsprobleme, Nachr. v. d. Ges. d. Wiss. zu Göttingen, Math-phys. Klasse 1918, 235 (1918).

[26] Particle Data Group, Review of Particle Physics, Phys. Rev. D 86, 010001 (2012), and 2013 partial update for the 2014 edition.

[27] C. S. Wu, E. Ambler, R. W. Hayward, D. D. Hoppes, and R. P. Hudson, Experimental Test of Parity Conservation in Beta Decay, Phys. Rev. 105, 1413 (1957).

[28] Gargamelle Collaboration, Observation of neutrino-like interactions without muon or electron in the gargamelle neutrino experiment, Phys. Lett. B 46, 138 (1973).

[29] L. Alvarez-Gaume and J. Ellis, Eyes on a prize particle, Nature Phys. 7, 2 (2011).

[30] J. Goldstone, A. Salam, and S. Weinberg, Broken Symmetries, Phys. Rev. 127, 965 (1962).

[31] F. Englert and R. Brout, Broken Symmetry and the Mass of Gauge Vector Mesons, Phys. Rev. Lett. 13, 321 (1964).

[32] P. W. Higgs, Broken symmetries, massless particles and gauge fields, Phys. Lett. 12, 132 (1964).

[33] P. W. Higgs, Broken Symmetries and the Masses of Gauge Bosons, Phys. Rev. Lett. 13, 508 (1964).

[34] G. S. Guralnik, C. R. Hagen, and T. W. B. Kibble, Global Conservation Laws and Massless Particles, Phys. Rev. Lett. 13, 585 (1964).

[35] P. W. Higgs, Spontaneous Symmetry Breakdown without Massless Bosons, Phys. 
Rev. 145, 1156 (1966).

[36] T. W. B. Kibble, Symmetry Breaking in Non-Abelian Gauge Theories, Phys. Rev. 155, 1554 (1967).

[37] UA1 Collaboration, Experimental observation of isolated large transverse energy electrons with associated missing energy at $\sqrt{s}=540$ GeV, Phys. Lett. B 122, 103 (1983).

[38] UA2 Collaboration, Observation of single isolated electrons of high transverse momentum in events with missing transverse energy at the CERN $\bar{p} p$ collider, Phys. Lett. B 122, 476 (1983).

[39] UA1 Collaboration, Experimental observation of lepton pairs of invariant mass around $95 \mathrm{GeV} / \mathrm{c}^{2}$ at the CERN SPS collider, Phys. Lett. B 126, 398 (1983).

[40] UA2 Collaboration, Evidence for $Z^{0} \rightarrow e^{+} e^{-}$at the CERN $\bar{p} p$ collider, Phys. Lett. B 129, 130 (1983).

[41] UA1 Collaboration, Studies of intermediate vector boson production and decay in UA1 at the CERN proton-antiproton collider, Z. Phys. C 44, 15 (1989).

[42] P. B. Littlewood and C. M. Varma, Gauge-Invariant Theory of the Dynamical Interaction of Charge Density Waves and Superconductivity, Phys. Rev. Lett. 47, 811 (1981).

[43] LHC Higgs Cross Section Working Group, Handbook of LHC Higgs Cross Sections: 2. Differential Distributions, CERN-2012-002 (2012), arXiv:1201.3084 [hep-ph].

[44] Particle Data Group, Review of Particle Physics, Phys. Lett. B 204, 1 (1988).

[45] ALEPH, DELPHI, L3, OPAL Collaborations, LEP Working Group for Higgs boson searches, Search for the Standard Model Higgs boson at LEP, Phys. Lett. B 565, 61 (2003).

[46] CDF, D0 Collaborations, Tevatron New Phenomena and Higgs Working Group, Combined CDF and DO Upper Limits on Standard Model Higgs-Boson Production with up to $6.7 \mathrm{fb}^{-1}$ of Data, FERMILAB-CONF-10-257-E (2010), arXiv:1007.4587 [hep-ex].

[47] H. Flacher et al., Revisiting the Global Electroweak Fit of the Standard Model and 
Beyond with Gfitter, Eur. Phys. J. C 60, 543 (2009), arXiv:0811.0009 [hep-ph], updated results taken from http://cern.ch/gfitter (Aug 10).

[48] J. Ellis, J. R. Espinosa, G. F. Giudice, A. Hoecker, and A. Riotto, The Probable Fate of the Standard Model, Phys. Lett. B 679, 369 (2009), arXiv:0906.0954 [hep-ph].

[49] ATLAS, CDF, CMS and D0 Collaborations, First combination of Tevatron and LHC measurements of the top-quark mass, ATLAS-CONF-2014-008 (2014), arXiv:1403.4427 [hep-ex].

[50] R. K. Ellis, W. J. Stirling, and B. R. Webber, QCD and Collider Physics (Cambridge University Press, Cambridge, UK, 1996).

[51] K. G. Wilson, Confinement of quarks, Phys. Rev. D 10, 2445 (1974).

[52] D. J. Gross and F. Wilczek, Ultraviolet Behavior of Non-Abelian Gauge Theories, Phys. Rev. Lett. 30, 1343 (1973).

[53] H. D. Politzer, Reliable Perturbative Results for Strong Interactions?, Phys. Rev. Lett. 30, 1346 (1973).

[54] J. C. Collins and D. E. Soper, Parton distribution and decay functions, Nucl. Phys. B 194, 445 (1982).

[55] V. N. Gribov and L. N. Lipatov, Deep inelastic ep scattering in perturbation theory, Sov. J. Nucl. Phys. 15, 438 (1972).

[56] G. Altarelli and G. Parisi, Asymptotic freedom in parton language, Nucl. Phys. B 126, 298 (1977).

[57] Y. L. Dokshitser, Calculation of Structure Functions of Deep Inelastic Scattering and $e^{+} e^{-}$Annihilation by Perturbative Theory in Quantum Chromodynamics, Sov. Phys.-JETP 46, 641 (1977).

[58] A. D. Martin, W. J. Stirling, R. S. Thorne, and G. Watt, Parton distributions for the LHC, Eur. Phys. J. C 63, 189 (2009), arXiv:0901.0002 [hep-ph].

[59] A. Buckley et al., General-purpose event generators for LHC physics, Phys. Rep. 504, 145 (2011), arXiv:1101.2599 [hep-ph].

[60] S. Höche, Matching to Matrix Elements, in MCnet-LPCC Summer School on Monte Carlo Event Generators for LHC, Geneva, 2012. 
[61] M. R. Whalley, D. Bourilkov, and R. C. Group, The Les Houches accord PDFs (LHAPDF) and LHAGLUE, in HERA and the LHC, Hamburg, 2005, arXiv:hep-ph/0508110.

[62] H.-L. Lai et al., New parton distributions for collider physics, Phys. Rev. D 82, 074024 (2010), arXiv:1007.2241 [hep-ph].

[63] R. D. Ball et al., Parton distributions with LHC data, Nucl. Phys. B 867, 244 (2013), arXiv:1207.1303 [hep-ph].

[64] G. Corcella et al., HERWIG 6: an event generator for hadron emission reactions with interfering gluons (including supersymmetric processes), JHEP 0101, 010 (2001), arXiv:hep-ph/0210213.

[65] M. Bähr et al., Herwig++ physics and manual, Eur. Phys. J. C 58, 639 (2008), arXiv:0803.0883 [hep-ph].

[66] J. M. Butterworth, J. R. Forshaw, and M. H. Seymour, Multiparton interactions in photoproduction at HERA, Z. Phys. C 72, 637 (1996), arXiv:hep-ph/9601371.

[67] T. Sjöstrand, S. Mrenna, and P. Skands, PYTHIA 6.4 physics and manual, JHEP 0605, 026 (2006), arXiv:hep-ph/0603175.

[68] T. Sjöstrand, S. Mrenna, and P. Skands, A brief introduction to PYTHIA 8.1, Computer Phys. Comm. 178, 852 (2008), arXiv:0710.3820 [hep-ph].

[69] T. Gleisberg et al., Event generation with SHERPA 1.1, JHEP 0902, 007 (2009), arXiv:0811.4622 [hep-ph].

[70] S. Catani, F. Krauss, R. Kuhn, and B. R. Webber, QCD Matrix eElements + Parton Showers, JHEP 0111, 063 (2001), arXiv: hep-ph/0109231.

[71] L. Lonnblad, Correcting the Color-Dipole Cascade Model with Fixed Order Matrix Elements, JHEP 0205, 046 (2002), arXiv:hep-ph/0112284.

[72] S. Hoeche et al., Matching Parton Showers and Matrix Elements, (2006), arXiv:hep-ph/0602031.

[73] M. L. Mangano, M. Moretti, F. Piccinini, R. Pittau, and A. D. Polosa, ALPGEN, a generator for hard multiparton processes in hadronic collisions, JHEP 0307, 001 (2003), arXiv:hep-ph/0206293.

[74] J. Alwall, M. Herquet, F. Maltoni, O. Mattelaer, and T. Stelzer, MadGraph 5: 
going beyond, JHEP 1106, 128 (2011), arXiv:1106.0522 [hep-ph].

[75] P. Nason and B. Webber, Next-to-Leading-Order Event Generators, Ann. Rev. Nucl. Part. Sci. 62, 187 (2012), arXiv:1202.1251 [hep-ph].

[76] S. Frixione and B. R. Webber, Matching NLO QCD computations and parton shower simulations, JHEP 0206, 029 (2002), arXiv:hep-ph/0204244.

[77] S. Frixione, F. Stoeckli, P. Torrielli, and B. R. Webber, NLO QCD corrections in Herwig++with MC@NLO, JHEP 1101, 053 (2011), arXiv:1010.0568 [hep-ph].

[78] V. Hirschi et al., Automation of one-loop QCD corrections, JHEP 1105, 044 (2011), arXiv:1103.0621 [hep-ph].

[79] P. Torrielli and S. Frixione, Matching NLO QCD computations with PYTHIA using MC@NLO, JHEP 1004, 110 (2010), arXiv:1002.4293 [hep-ph].

[80] P. Nason, A new method for combining NLO QCD with shower Monte Carlo algorithms, JHEP 0411, 040 (2004), arXiv:hep-ph/0409146.

[81] S. Frixione, P. Nason, and C. Oleari, Matching NLO QCD computations with Parton Shower simulations: the POWHEG method, JHEP 0711, 070 (2007), arXiv:0709.2092 [hep-ph].

[82] S. Alioli, P. Nason, C. Oleari, and E. Re, A general framework for implementing NLO calculations in shower Monte Carlo programs: the POWHEG BOX, JHEP 1006, 043 (2010), arXiv:1002.2581 [hep-ph].

[83] S. Agostinelli et al., Geant4 - a simulation toolkit, Nucl. Instrum. Methods A506, 250 (2003).

[84] ATLAS Collaboration, The ATLAS Simulation Infrastructure, Eur. Phys. J. C 70, 823 (2010), arXiv:1005.4568 [physics.ins-det].

[85] ATLAS Collaboration, The simulation principle and performance of the ATLAS fast calorimeter simulation FastCaloSim, ATL-PHYS-PUB-2010-013 (2010).

[86] ATLAS Collaboration, Summary of ATLAS Pythia 8 tunes, ATL-PHYS-PUB-2012-003 (2012).

[87] ATLAS Collaboration, Example ATLAS tunes of Pythia8, Pythia6 and Powheg to an observable sensitive to $Z$ boson transverse momentum, 
ATL-PHYS-PUB-2013-017 (2013).

[88] G. P. Salam, Towards Jetography, Eur. Phys. J. C 67, 637 (2010), arXiv:0906.1833 [hep-ph].

[89] M. Cacciari, G. P. Salam, and G. Soyez, FastJet User Manual, Eur. Phys. J. C 72, 1896 (2012), arXiv:1111.6097 [hep-ph].

[90] M. Cacciari, G. P. Salam, and G. Soyez, The anti-k $k_{t}$ jet clustering algorithm, JHEP 0804, 063 (2008), arXiv:0802.1189 [hep-ph].

[91] L. Evans and P. Bryant, LHC Machine, JINST 3, S08001 (2008).

[92] ATLAS Collaboration, Improved luminosity determination in pp collisions at $\sqrt{s}=7$ TeV using the ATLAS detector at the LHC, Eur. Phys. J. C 73, 1 (2013), arXiv:1302.4393 [hep-ex].

[93] ATLAS Collaboration, The ATLAS Experiment at the CERN Large Hadron Collider, JINST 3, S08003 (2008).

[94] ATLAS Collaboration, K. Nagano, Algorithms, performance, and development of the ATLAS High-level Trigger, ATL-DAQ-SLIDE-2013-893 (2013).

[95] ATLAS Collaboration, Alignment of the ATLAS Inner Detector and its Performance in 2012, ATLAS-CONF-2014-047 (2014).

[96] ATLAS Collaboration, Electron and photon energy calibration with the ATLAS detector using LHC Run 1 data, (2014), arXiv:1407.5063 [hep-ex], submitted to Eur. Phys. J. C.

[97] ATLAS Collaboration, Jet momentum resolution with the ATLAS detector in proton-proton collisions at $\sqrt{s}=8$ TeV recorded in 2012, ATL-COM-PHYS-2014-010 (2014), (ATLAS internal).

[98] ATLAS Collaboration, Measurement of the muon reconstruction performance of the ATLAS detector using 2011 and 2012 LHC proton-proton collision data, (2014), arXiv:1407.3935 [hep-ex], submitted to Eur. Phys. J. C.

[99] T. Cornelissen et al., The new ATLAS track reconstruction (NEWT), J. Phys.: Conf. Ser. 119, 032014 (2008).

[100] ATLAS Collaboration, Expected Performance of the ATLAS Experiment Detector, Trigger and Physics, CERN-OPEN-2008-020 (2008), arXiv:0901.0512 
[hep-ex] .

[101] G. Piacquadio, K. Prokofiev, and A. Wildauer, Primary vertex reconstruction in the ATLAS experiment at LHC, J. Phys.: Conf. Ser. 119, 032033 (2008).

[102] E. Bouhova-Thacker et al., Expected Performance of Vertex Reconstruction in the ATLAS Experiment at the LHC, IEEE Trans. Nucl. Sci. 57, 760 (2010).

[103] ATLAS Collaboration, Expected electron performance in the ATLAS experiment, ATL-PHYS-PUB-2011-006 (2011).

[104] ATLAS Collaboration, Improved electron reconstruction in ATLAS using the Gaussian Sum Filter-based model for bremsstrahlung, ATLAS-CONF-2012-047 (2012).

[105] ATLAS Collaboration, Electron performance measurements with the ATLAS detector using the 2010 LHC proton-proton collision data, Eur. Phys. J. C 72, 1909 (2012), arXiv:1110.3174 [hep-ex].

[106] S. Laplace and J. de Vivie, Calorimeter isolation and pile-up, ATL-COM-PHYS-2012-467 (2012), (ATLAS internal).

[107] ATLAS Collaboration, Electron Efficiency Measurements for 2012 and 2011 Data, ATL-COM-PHYS-2013-1287 (2013).

[108] R. Nicolaidou et al., Muon identification procedure for the ATLAS detector at the LHC using Muonboy reconstruction package and tests of its performance using cosmic rays and single beam data, J. Phys.: Conf. Ser. 219, 032052 (2010).

[109] ATLAS Collaboration, Preliminary results on the muon reconstruction efficiency, momentum resolution, and momentum scale in ATLAS 2012 pp collision data, ATLAS-CONF-2013-088 (2013).

[110] ATLAS Collaboration, Jet energy measurement with the ATLAS detector in proton-proton collisions at $\sqrt{s}=7$ TeV, Eur. Phys. J. C 73, 2304 (2013), arXiv:1112.6426 [hep-ex].

[111] ATLAS Collaboration, Jet energy scale and its systematic uncertainty in proton-proton collisions at $\sqrt{s}=7$ TeV with ATLAS 2011 data, ATLAS-CONF-2013-004 (2013).

[112] ATLAS Collaboration, Pile-up subtraction and suppression for jets in ATLAS, 
ATLAS-CONF-2013-083 (2013).

[113] ATLAS Collaboration, https://twiki.cern.ch/twiki/bin/view/AtlasPublic/ JetEtmissApproved2013JESUncertainty, retrieved 21/05/14.

[114] ATLAS Collaboration, Selection of jets produced in proton-proton collisions with the ATLAS detector using 2011 data, ATLAS-CONF-2012-020 (2012).

[115] ATLAS Collaboration, Commissioning of the ATLAS high-performance b-tagging algorithms in the 7 TeV collision data, ATLAS-CONF-2011-102 (2011).

[116] ATLAS Collaboration, Calibration of b-tagging using dileptonic top pair events in a combinatorial likelihood approach with the ATLAS experiment,

ATLAS-CONF-2014-004 (2014).

[117] ATLAS Collaboration, Performance of Missing Transverse Momentum

Reconstruction in ATLAS studied in Proton-Proton Collisions recorded in 2012 at $8 \mathrm{TeV}$, ATLAS-CONF-2013-082 (2013).

[118] ATLAS Collaboration, Measurements of the photon identification efficiency with the ATLAS detector using $4.9 \mathrm{fb}^{-1}$ of pp collision data collected in 2011, ATLAS-CONF-2012-123 (2012).

[119] ATLAS Collaboration, Determination of the tau energy scale and the associated systematic uncertainty in proton-proton collisions at $\sqrt{s}=8$ TeV with the ATLAS detector at the LHC in 2012, ATLAS-CONF-2013-044 (2013).

[120] ATLAS Collaboration, https://twiki.cern.ch/twiki/bin/view/AtlasPublic/ MuonTriggerPublicResults, retrieved 21/05/14.

[121] R. K. Ellis, I. Hinchliffe, M. Soldate, and J. J. Van Der Bij, Higgs decay to $\tau^{+} \tau^{-}$: A possible signature of intermediate mass Higgs bosons at high energy hadron colliders, Nucl. Phys. B 297, 221 (1988).

[122] A. Hoecker et al., TMVA: Toolkit for Multivariate Data Analysis, PoS ACAT, 040 (2007), arXiv:physics/0703039.

[123] R. Boughezal, X. Liu, F. Petriello, F. J. Tackmann, and J. R. Walsh, Combining Resummed Higgs Predictions Across Jet Bins, Phys. Rev. D 89, 074044 (2014), arXiv:1312.4535 [hep-ph].

[124] I. W. Stewart and F. J. Tackmann, Theory uncertainties for Higgs and other 
searches using jet bins, Phys. Rev. D 85, 034011 (2012), arXiv:1107.2117 [hep-ph].

[125] M. Grazzini, NNLO predictions for the Higgs boson signal in the $H \rightarrow W W \rightarrow \ell \nu \ell \nu$ and $H \rightarrow Z Z \rightarrow 4 \ell$ decay channels, JHEP 0802, 043 (2008), arXiv:0801.3232 [hep-ph].

[126] A. Banfi, G. P. Salam, and G. Zanderighi, NLL+NNLO predictions for jet-veto efficiencies in Higgs-boson and Drell-Yan production, JHEP 1206, 159 (2012), arXiv:1203.5773 [hep-ph].

[127] A. Banfi, P. F. Monni, G. P. Salam, and G. Zanderighi, Higgs and Z-boson production with a jet veto, Phys. Rev. Lett. 109, 202001 (2012), arXiv:1206.4998 [hep-ph].

[128] J. M. Campbell, R. K. Ellis, and C. Williams, Hadronic production of a Higgs boson and two jets at next-to-leading order, Phys. Rev. D 81, 074023 (2010), arXiv:1001.4495 [hep-ph].

[129] R. Boughezal, F. Caola, K. Melnikov, F. Petriello, and M. Schulze, Higgs boson production in association with a jet at next-to-next-to-leading order in perturbative QCD, JHEP 1306, 072 (2013), arXiv:1302.6216 [hep-ph].

[130] E. Bagnaschi, G. Degrassi, P. Slavich, and A. Vicini, Higgs production via gluon fusion in the POWHEG approach in the SM and in the MSSM, JHEP 1202, 088 (2012), arXiv:1111.2854 [hep-ph].

[131] D. de Florian, G. Ferrera, M. Grazzini, and D. Tommasini, Transverse-momentum resummation: Higgs boson production at the Tevatron and the LHC, JHEP 1111, 064 (2011), arXiv:1109.2109 [hep-ph].

[132] M. Grazzini and H. Sargsyan, Heavy-quark mass effects in Higgs boson production at the LHC, JHEP 1309, 129 (2013), arXiv:1306.4581 [hep-ph].

[133] K. Hamilton, P. Nason, and G. Zanderighi, MINLO: Multi-Scale Improved NLO, JHEP 1210, 155 (2012), arXiv:1206.3572 [hep-ph].

[134] ATLAS Collaboration, Pile-up corrections for jets from proton-proton collisions at $\sqrt{s}=7$ TeV in ATLAS in 2011, ATLAS-CONF-2012-064 (2012).

[135] T. Binoth, M. Ciccolini, N. Kauer, and M. Kramer, Gluon-induced W-boson pair production at the LHC, JHEP 0612, 046 (2006), arXiv:hep-ph/0611170. 
[136] ATLAS Collaboration, Jet energy resolution in proton-proton collisions at $\sqrt{s}=7$ TeV recorded in 2010 with the ATLAS detector, Eur. Phys. J. C 73, 2306 (2013), arXiv:1210.6210 [hep-ex].

[137] J. M. Campbell, R. K. Ellis, and C. Williams, Vector boson pair production at the LHC, JHEP 1107, 018 (2011), arXiv:1105.0020 [hep-ph].

[138] ATLAS Collaboration, Further ATLAS tunes of PYTHIA6 and Pythia 8, ATL-PHYS-PUB-2011-014 (2011).

[139] P. Z. Skands, Tuning Monte Carlo Generators: The Perugia Tunes, Phys. Rev. D 82, 074018 (2010), arXiv:1005.3457 [hep-ph].

[140] A. Bierweiler, T. Kasprzik, J. H. Kühn, and S. Uccirati, Electroweak corrections to W-boson pair production at the LHC, JHEP 1211, 093 (2012), arXiv: 1208.3147 [hep-ph].

[141] ATLAS Collaboration, Background estimation in the $H \rightarrow W W^{(*)} \rightarrow \ell \nu \ell \nu$ analysis with $20.7 \mathrm{fb}^{-1}$ of data collected with the ATLAS detector at $\sqrt{\mathrm{s}}=8 \mathrm{TeV}$, ATL-COM-PHYS-2013-1630 (2013), (ATLAS internal).

[142] J. Alwall, private communication, 2012.

[143] F. Krauss and F. Siegert, private communication, 2012.

[144] ROOT Collaboration, K. Cranmer, G. Lewis, L. Moneta, A. Shibata, and W. Verkerke, HistFactory: A tool for creating statistical models for use with RooFit and RooStats, CERN-OPEN-2012-016 (2012).

[145] G. Cowan, K. Cranmer, E. Gross, and O. Vitells, Asymptotic formulae for likelihood-based tests of new physics, Eur. Phys. J. C 71, 1554 (2011), arXiv:1007.1727 [physics.data-an].

[146] K. Cranmer, Practical Statistics for the LHC, in 7th CERN-Latin American School of High-Energy Physics, Arequipa, Peru, 2013.

[147] T. Junk, Confidence level computation for combining searches with small statistics, Nucl. Instrum. Methods A434, 435 (1999).

[148] A. L. Read, Presentation of search results: the $C L_{s}$ technique, J. Phys. G: Nucl. and Part. Phys. 28, 2693 (2002).

[149] CMS Collaboration, Measurement of Higgs boson production and properties in the 
WW decay channel with leptonic final states, JHEP 1401, 096 (2014), arXiv:1312.1129 [hep-ex].

[150] CMS Collaboration, Observation of a new boson at a mass of $125 \mathrm{GeV}$ with the CMS experiment at the LHC, Phys. Lett. B 716, 30 (2012), arXiv:1207.7235 [hep-ex].

[151] CDF, D0 Collaborations, Evidence for a Particle Produced in Association with Weak Bosons and Decaying to a Bottom-Antibottom Quark Pair in Higgs Boson Searches at the Tevatron, Phys. Rev. Lett. 109, 071804 (2012), arXiv:1207.6436 [hep-ex].

[152] ATLAS Collaboration, Measurement of Higgs boson production in the diphoton decay channel in pp collisions at center-of-mass energies of 7 and $8 \mathrm{TeV}$ with the ATLAS detector, (2014), arXiv:1408.7084 [hep-ex], submitted to Phys. Rev. D.

[153] ATLAS Collaboration, Measurements of Higgs boson production and couplings in the four-lepton channel in pp collisions at center-of-mass energies of 7 and 8 TeV with the ATLAS detector, (2014), arXiv:1408.5191 [hep-ex], submitted to Phys. Rev. D.

[154] ATLAS Collaboration, Measurement of the Higgs boson mass from the $H \rightarrow \gamma \gamma$ and $H \rightarrow Z Z^{*} \rightarrow 4 \ell$ channels with the ATLAS detector using $25 \mathrm{fb}^{-1}$ of $p p$ collision data, (2014), arXiv:1406.3827 [hep-ex], accepted by Phys. Rev. D.

[155] CMS Collaboration, Combination of standard model Higgs boson searches and measurements of the properties of the new boson with a mass near $125 \mathrm{GeV}$, CMS-PAS-HIG-13-005 (2013).

[156] CMS Collaboration, Observation of the diphoton decay of the Higgs boson and measurement of its properties, (2014), arXiv:1407.0558 [hep-ex], submitted to Eur. Phys. J. C.

[157] CMS Collaboration, Measurement of the properties of a Higgs boson in the four-lepton final state, Phys. Rev. D 89, 092007 (2014), arXiv:1312.5353 [hep-ex].

[158] CMS Collaboration, Evidence for the 125 GeV Higgs boson decaying to a pair of $\tau$ leptons, JHEP 1405, 104 (2014), arXiv:1401.5041 [hep-ex]. 
[159] CMS Collaboration, Search for the standard model Higgs boson produced in association with a $W$ or a $Z$ boson and decaying to bottom quarks, Phys. Rev. D 89, 012003 (2014), arXiv:1310.3687 [hep-ex].

[160] ATLAS Collaboration, Updated coupling measurements of the Higgs boson with the ATLAS detector using up to $25 \mathrm{fb}^{-1}$ of proton-proton collision data, ATLAS-CONF-2014-009 (2014).

[161] ATLAS Collaboration, Search for Higgs boson decays to a photon and a $Z$ boson in $p p$ collisions at $\sqrt{s}=7$ and 8 TeV with the ATLAS detector, Phys. Lett. B 732, 8 (2014), arXiv:1402.3051 [hep-ex].

[162] ATLAS Collaboration, Search for the Standard Model Higgs boson decay to $\mu^{+} \mu^{-}$ with the ATLAS detector, (2014), arXiv:1406.7663 [hep-ex], accepted by Phys. Lett. B.

[163] ATLAS Collaboration, Search for Invisible Decays of a Higgs Boson Produced in Association with a Z Boson in ATLAS, Phys. Rev. Lett. 112, 201802 (2014), arXiv:1402.3244 [hep-ex].

[164] CMS Collaboration, Search for a Higgs boson decaying into a $Z$ and a photon in pp collisions at $\sqrt{s}=7$ and 8 TeV, Phys. Lett. B 726, 587 (2013), arXiv:1307.5515 [hep-ex].

[165] CMS Collaboration, Search for the standard model Higgs boson in the dimuon decay channel in pp collisions at $\sqrt{s}=7$ and 8 TeV, CMS-PAS-HIG-13-007 (2013).

[166] CMS Collaboration, Search for invisible decays of Higgs bosons in the vector boson fusion and associated ZH production modes, Eur. Phys. J. C 74, 2980 (2014), arXiv:1404.1344 [hep-ex].

[167] L. D. Landau, On the angular momentum of a two-photon system, Dokl. Akad. Nauk Ser. Fiz. 60, 207 (1948).

[168] C.-N. Yang, Selection Rules for the Dematerialization of a Particle Into Two Photons, Phys. Rev. 77, 242 (1950).

[169] ATLAS Collaboration, Evidence for the spin-0 nature of the Higgs boson using ATLAS data, Phys. Lett. B 726, 120 (2013), arXiv:1307.1432 [hep-ex].

[170] M. Baak et al., The electroweak fit of the standard model after the discovery of a 
new boson at the LHC, Eur. Phys. J. C 72, 1 (2012), arXiv:1209.2716

[hep-ph], updated results taken from http://cern.ch/gfitter (Sep 13).

[171] D. Buttazzo et al., Investigating the near-criticality of the Higgs boson, JHEP 1312, 089 (2013), arXiv:1307.3536.

[172] G. Isidori, V. S. Rychkov, A. Strumia, and N. Tetradis, Gravitational corrections to standard model vacuum decay, Phys. Rev. D 77, 025034 (2008), arXiv:0712.0242 [hep-ph].

[173] A. De Simone, M. P. Hertzberg, and F. Wilczek, Running inflation in the Standard Model, Phys. Lett. B 678, 1 (2009), arXiv:0812.4946 [hep-ph].

[174] S. P. Martin, A Supersymmetry primer, (1997), arXiv:hep-ph/9709356.

[175] M. E. Peskin, Beyond the standard model, (1997), arXiv:hep-ph/9705479.

[176] A. Pomarol, Beyond the Standard Model, (2012), arXiv:1202.1391 [hep-ph].

[177] ATLAS Collaboration, Projections for measurements of Higgs boson cross sections, branching ratios and coupling parameters with the ATLAS detector at a HL-LHC, ATL-PHYS-PUB-2013-014 (2013).

[178] F. Caola and K. Melnikov, Constraining the Higgs boson width with ZZ production at the LHC, Phys. Rev. D 88, 054024 (2013), arXiv:1307.4935 [hep-ph].

[179] J. M. Campbell, R. K. Ellis, and C. Williams, Bounding the Higgs width at the LHC using full analytic results for $g g \rightarrow e^{-} e^{+} \mu^{-} \mu^{+}$, JHEP 1404, 060 (2014), arXiv:1311.3589 [hep-ph].

[180] J. M. Campbell, R. K. Ellis, and C. Williams, Bounding the Higgs width at the LHC: complementary results from $H \rightarrow W W$, Phys. Rev. D 89, 053011 (2014), arXiv:1312.1628 [hep-ph].

[181] L. J. Dixon and Y. Li, Bounding the Higgs Boson Width Through Interferometry, Phys. Rev. Lett. 111, 111802 (2013), arXiv:1305.3854 [hep-ph].

[182] S. P. Martin, Interference of Higgs diphoton signal and background in production with a jet at the LHC, Phys. Rev. D 88, 013004 (2013), arXiv:1303.3342 [hep-ph].

[183] M. J. Dolan, C. Englert, and M. Spannowsky, Higgs self-coupling measurements at the LHC, JHEP 1210, 112 (2012), arXiv:1206.5001 [hep-ph]. 